\title{
Influência do Campo de
Chern-Simons Sobre as Propriedades \\ Influência do Campo de
Chern-Simons Sobre as Propriedades de Renormalizabilidade do Modelo $\left(\phi^{*} \phi\right)^{3}$
}

Sérgio Vizeu Lima Pinheiro

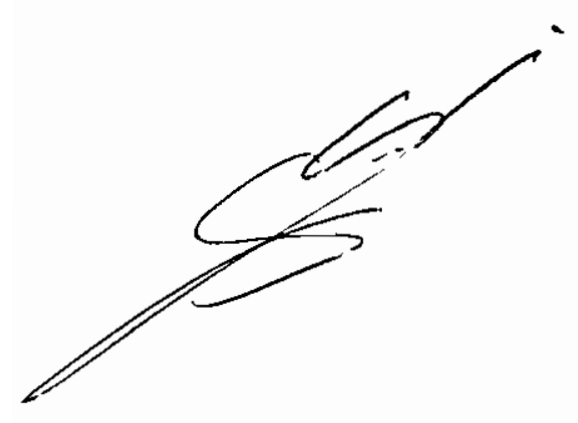

Tese de Doutorado

submetida ao Instituto de Física da Universidade de São Paulo

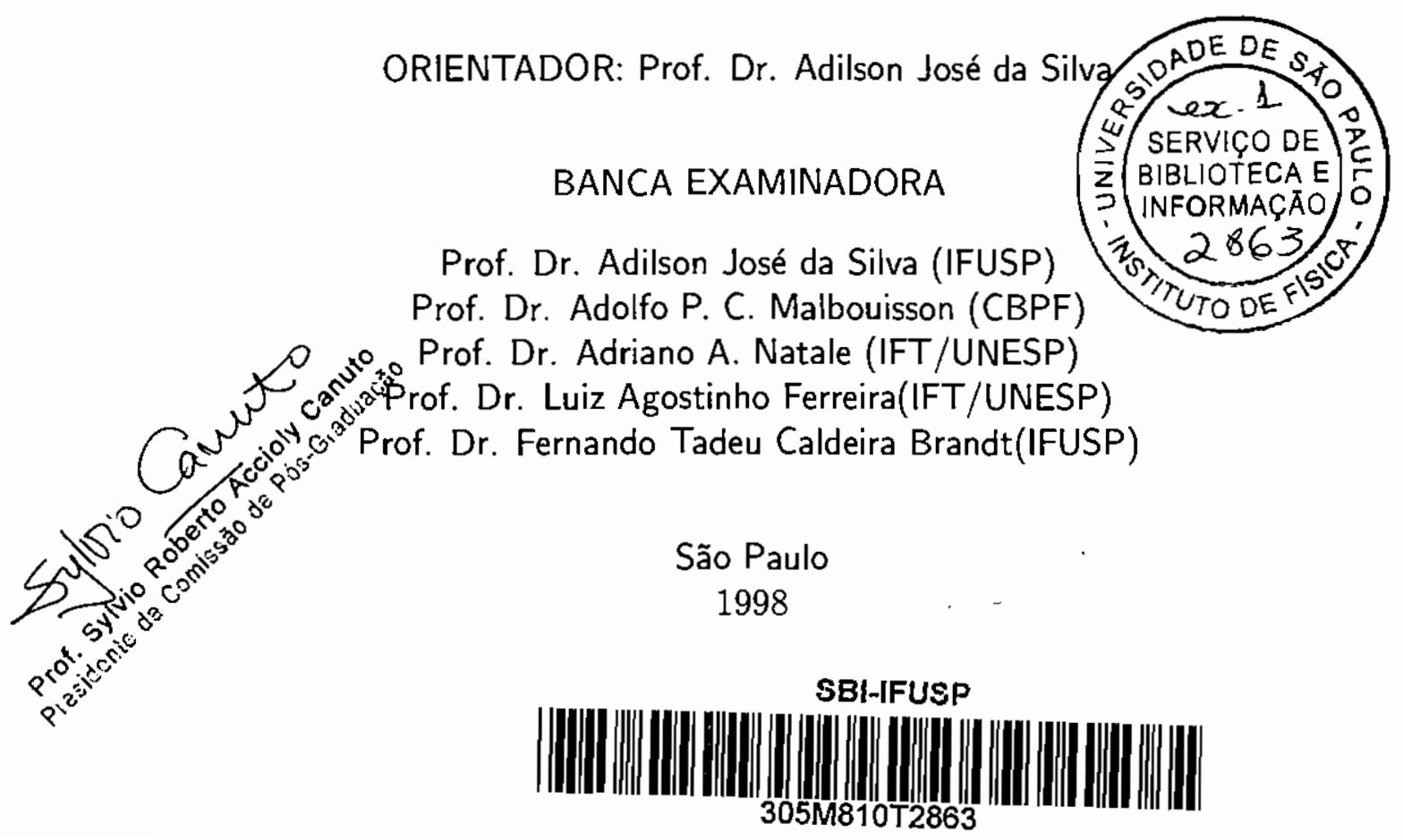




\section{FICHA CATALOGRÁFICA}

Preparada pelo Serviço de Biblioteca e Informação do Instituto de Física da Universidade de São Paulo

Pinheiro, Sérgio Vizeu Lima

Influência do Campo de Chern-Simons sobre as Propriedades de Renormalizabilidade do Modelo $\left(\varnothing^{*} \varnothing\right)^{3}$. São Paulo, 1998.

Tese (Doutoramento) - Universidade de São Paulo. Instituto de Física - Departamento de Física Matemática

Orientador: Prof. Dr. Adilson José da Silva Área de Concentração: Física de Particulas e Campos

Unitermos: 1 . Teoria de Campos; 2. Modelo $\left(\varnothing^{*} \varnothing\right)^{3}$; 3. Grupo de Renormalização; 4. Chern-Simons. 
ABSTRACT

We analyse the effect of coupling of a Chern-Simons field on the renormalization properties of the model $\left(\phi^{*} \phi\right)^{3}$ and of the composite operators $\left[\left(\phi^{*} \phi\right)^{n}\right]$ in $2+1$ dimensions. For that, we calculate the two and six point vertex functions up to respectively second and third order in the coupling constants, using dimensional regularization and minimal subtraction. Renormalization group equations. the $\beta$ functions and the anomalous dimensions of the basic field $\phi$ and composite fields $\left[\left(\phi^{*} \phi\right)^{n}\right]$ were got. It is show that the coupling to the Chern-Simons generate a phase in which asymptotic freedom is possible. 


\section{RESUMO}

Neste trabalho analisamos a influência do campo de Chern-Simons sobre as propriedades de renormalizabilidade do modelo $\left(\phi^{*} \phi\right)^{3}$, e dos operadores compostos $\left[\left(\phi^{*} \phi\right)^{n}\right] \mathrm{em}$ $2+1$ dimensōes. Para tanto calcularemos as funçōes de vértice de dois e seis pontos até as ordens de perturbação dois e três, respectivamente. Foi usado o método de contratermos para renormalizar as funções de Green, e a regularização dimensional para isolar as divergências nos diagramas de Feynman. Foi deduzida a equação do grupo de renormalização e obtida a função beta e a dimensão anômala para o campo básico. Foi também obtida e analisada a dimensão anômala para o operador composto $\left[\left(\phi^{*} \phi\right)^{n}\right]$. Todas a análises foram feitas no sentido de obter a influência do acoplamento de Chern-Simons sobre as funções do grupo de renormalização. 
Dedicatória, Aos meus pais, Cezídio e Maria de Belém. 


\section{Agradecimentos}

- Ao Prof. Dr. Adilson J. da Silva pela orientação;

- Aos meus pais e irmãos pelo incentivo e carinho de todas as horas;

- Aos companheiros de labuta, do cotidiano das contas, das discussões, dos dias de desânimo e, como não poderia deixar de ser, de muitas alegrias também; Van Sérgio Alves, Luís Carlos Malacarne e Francisco Peña Campos;

- Aos muitos amigos que, perto ou longe, paticiparam destes anos e estão, de várias formas, presentes entre cada uma das contas deste trabalho; Soraia, Crispino, Angela, Silvana, Madalena. Alexandra, Marília. Macarena, Patrícia. Marcelo. Mazá, Geysa. Rita, D. Ady, Edna. Waní, Lene, Carla, Mel. Ana Flávia. Nara. Paulo Renato, Cida, Zu. Márcia, Castiñeira. Rênio, Rô, Ruy, Eliney. Valéria. Andréa, Buffon, Zé Roberto, Jorge, André, Zé Guilherme, Dete. Regina, Marco Antônio, Alexandre. Akira, Luis Cláudio. Edgar e Cleber;

- Ao Prof. Dr. Marcelo O. C. Gomes: pelas discussões e ajudas sempre bem-vindas;

- Aos amigos da secretaria; Amélia. Kelma e João ;

- Ao Departamento de Física da Universidade Federal do Pará. nas figuras da Profa. Fátima Baraúna, Paulo de Tarso Alencar, e em especial ao Prof. Dr. José Maria Filardo Bassalo, senipre amigo e professor:

- A CAPES pelo apoio financeiro. 


\section{Conteúdo}

$\begin{array}{lr}\text { Introdução } & 8\end{array}$

1 Propriedades Gerais do Modelo 11

1.1 Introdução . . . . . . . . . . . . . . . . . . . . 11

1.2 Apresentação do Modelo . . . . . . . . . . . . . . . . . . . . 11

1.3 Regras de Feynman para a Teoria Livre . . . . . . . . . . . . . . . . 12

1.4 O Grau de Divergência Superficial . . . . . . . . . . . . . . . . . 15

1.5 Transformações de Simetria . . . . . . . . . . . . . . . . . 17

1.6 Renormalização do Modelo . . . . . . . . . . . . . . . . . . . . . . . . . 19

1.6.1 O Método de Contratermos . . . . . . . . . . . . . . 20

1.6.2 Relações entre as Constantes de Renormalização . . . . . . . . . . 24

1.7 O Grupo de Renormalização . . . . . . . . . . . . . . . . . . 26

2 O Cálculo das Funções de Dois e Seis Pontos 32

2.1 Introdução . . . . . . . . . . . . . . . . . . . 32

2.2 Definições das Funções de Vértice de Dois e Seis Pontos . . . . . . . . . . . 33

2.3 Sub-diagramas . . . . . . . . . . . . . . . . . . . 34

2.4 A Função de Vértice de Dois Pontos . . . . . . . . . . . . . . . . . . 47

2.5 A Função de Vértice de Seis Pontos . . . . . . . . . . . . . . . . . 55

3 Análise das Funções do Grupo de Renormalização $\quad 77$

3.1 Introdução . . . . . . . . . . . . . . . . . . . . 77

3.2 A Função Beta: Pontos Fixos . . . . . . . . . . . . . . . . . . . . 77

3.3 Análise Dimensional da Equação do Grupo de Renormalização . . . . . . . 83

3.4 Dimensão Anômala do Campo $\phi$. . . . . . . . . . . . . . . . . . . . . . . 85

3.5 Operador Composto . . . . . . . . . . . . . . . . . . . . . 85

3.6 Equação do Grupo de Renormalização para o Operador Composto . . . . . 92

A Diagramas de Feynman $\quad 96$

A.1 Função de Dois Pontos . . . . . . . . . . . . . . . . . . . 996

A.2 Função de Seis Pontos . . . . . . . . . . . . . . . . . . . 98 
$\begin{array}{ll}\text { B Tabela } & 103\end{array}$

$\begin{array}{ll}\text { C Integrais Gerais dos Loops de Momentos } & 105\end{array}$

D Função de Vértice de Dois Pontos para o Campo $A_{\mu} \quad 108$

E Identidades de Ward 113

$\begin{array}{ll}\text { Bibliografia } & 121\end{array}$ 


\section{Introdução}

A conexão spin-estatística é largamente conhecida da mecânica quântica, ou seja, a classificação das partículas em férmions ou bósons dependendo se estas obedecem, respectivamente as estatísticas de Fermi-Dirac ou Bose-Einstein. Tal conexão manifesta-se na estrutura da função de onda; sendo estas simétricas ou anti-simétricas na permutação dos bósons ou férmions idênticos; tendo estes, por sua vez, spin inteiros (bósons) ou semi-inteiros (férmions) em cada caso.

J. M. Leinaas e J. Myrheim[1] propuseram uma visão alternativa a este comportamento dicotômico da natureza. Partindo de uma interessante propriedade inerente ao espaço de duas dimensões; ou seja, do fato deste espaço ser multiplamente conexo (enquanto em três dimensões o espaço é duplamente conexo), eles ampliaram as possibilidades de valores do spin para uma partícula. Constataram que em duas dimensões a estatística quântica não se limita apenas a bósons e férmions ; mas sim a um conjunto contínuo de valores variando entre estas possibilidades extremas. A estatística quântica, para este caso mais geral, fica definida por uma fase que varia entre 1 (bósons) e 0 (férmions), e as partículas que obedecem os valores intermediarios (spin fracionários) são chamadas de anyons.

Partindo da conjectura de existência de objetos que satisfazem esta "estatística exótica", foram desenvolvidos alguns trabalhos com o intuito de discutir a existência ou não de anyons na natureza. Um do primeiros esforços neste sentido deveu-se a Tsui, Stormer e Gossard[2], em 1982, ao descobrirem o Efeito Hall Fracionário, identificando quasi-partículas com número quântico não usuais (contribuições teóricas importantes para este trabalho foram apresentadas ainda por Haldane[3] e Halperin[4], ambos em 1983, e Laughlin[5] em 1984). Outra tentativa de identificação de anyons na natureza deve-se a Lykken, Sonnenschein e Weiss[6] em 1991, ao associarem anyons à supercondutividade a altas temperaturas (alguns trabalhos teóricos neste mesmo sentido podem ser vistos em $[7,8,9,10])$.

Em 1982, Wilczek[11] reforçou o resultado de Leinaas e Myrhhein sobre a conexão spinestatística para anyons baseado no efeito Aharonov-Bohm[12], no qual um campo escalar é acoplado minimamente a um campo de Chern-Simons; e relacionou tal comportamento à interação a um potencial vetor associado aos anvons. O próprio Wilczek, juntamente com Arovas e Shrieffer[13], em 1984, consolidaram a descrição da estatística via a introdução de um campo de gauge $A_{\mu}$ (e não pela simetria da função de onda). acoplado minimamente 
com o campo de matéria, e com sua "dinâmica" descrita por um termo de Chern-Simons.

Uma importante generalização da teoria de Chern-Simons em $(2+1)$ dimensões que vale ser mencionada deve-se a Schonfeld[14], em 1981, e a Deser, Jackiw e Templeton[15], em 1982, somando a este o termo de Maxwell, e propiciando, desta forma, a existência de massa para o campo de gauge sem quebra da invariância de gauge.

Recentemente foi conjecturado que o termo de Chern-Simons além de mudar o spin e a estatística, transmutando partículas fermiônicas para escalares (e vice-versa), poderia afetar a dimensão dos campos, influenciando as propriedades de renormalizabilidade da teoria, a qual está acoplado minimamente o campo de gauge. Esta idéia foi colocada em prática por W. Chen e M. Li[16] em 1993, calculando dimensões anômalas para os operadores compostos $(\bar{\psi} \psi)^{n}$ e $\left(\phi^{*} \phi\right)^{n}$ ornamentados com Chern-Simons, e obtendo valores críticos para os acoplamentos de Chern-Simons que fazem operadores não-renormalizáveis se tornarem renormalizáveis. Em 1995, W. Chen[17] obteve uma possível linha de pontos fixos estáveis infra-vermelho não-gaussiano, caracteristico de um modelo escalar tipo $\left(\phi^{*} \phi\right)^{2}$, para uma interação fermiônica quadrilinear, com férmions acoplados minimamente ao campo de Chern-Simons.

Estes últimos trabalhos nos motivaram a analisar mais cuidadosamente a influência do termo de Chern-Simons sobre as funções do grupo de renormalização . Para o caso fermiônico foi feita a análise perturbativa até dois "loops" para o modelo de Gross-Neveu acoplado a um campo de Chern-Simons[18], usando a regularização dimensional. Este trabalho indica um melhor comportamento no ultravioleta para as funções de Green dos operadores renormalizáveis (dimensão do operador igual a dimensão do espaço-tempo), e que para operadores com dimensão maior que a dimensão do espaço tempo (não renormalizáveis) as suas dimensões anômalas aumentam com o parâmetro de Chern-Simons. Ainda referente ao caso fermiônico foi feita uma generalização somando uma interação tipo Thirring ao modelo de Gross-Neveu[19] (neste caso temos um número $N$ de campos de matéria). Neste último trabalho foi verificado a existência de uma linha de pontos fixos infra-vermelhos diferente do trivial, contrariamente ao obtido para o caso que apenas a interação Gross-Neveu está presente. Com relação aos operadores compostos constatou-se que para operadores renormalizáveis também há indicação de um melhor comportamento no ultravioleta, e que para operadores não renormalizáveis uma combinação dos termos de mistura dos operadores podem levar ao mesmo comportamento.

Neste trabalho vamos analisar a influência do termo de Cheln-Simons sobre as propriedades de renormalizabilidade do modelo escalar $\left(\phi^{*} \phi\right)^{3}$, renormalizável em $(2+1)$ dimensōes (O modelo $\left(\phi^{*} \phi\right)^{2}$ é super-renormalizável nesta dimensão).

As propriedades das funções do grupo de renormalização para o modelo $\left(\phi^{*} \phi\right)^{3}$ tem despertado interesse nos últimos anos, e sendo analisadas sob vários aspectos, tanto diante de uma abordagem não perturbativa[20,21] (expansão 1/N), quanto diante de uma expansão perturbativa padrão[22]. Um aspecto importante que vale ser mencionado é a utilização da interação $\left(\phi^{*} \phi\right)^{3}$ em $(2+1)$ dimensões, na análise do fenômeno de tricriticalidade em misturas ${ }^{3} \mathrm{He}-{ }^{4} \mathrm{He}[23]$; mediante a utilização de método não perturbativos[24] 
ou perturbativos[25, 26].

O presente trabalho está dividido em três partes; no capítulo um vamos apresentar as propriedades gerais do modelo (simetrias, grau de divergência superficial, etc.), assim como discutiremos sua renormalização. No capítulo dois calcularemos as funções de vértice de dois e seis pontos até a terceira ordem de perturbação nas constantes de acoplamento, usando a regularização dimensional, e explicitaremos os contratermos necessários para absorver as divergências das funções de vértice. O terceiro capítulo será dedicado ao cálculo e análise das funções do grupo de renormalização para o campo básico, e da dimensão anômala dos operadores compostos $\left(\phi^{*} \phi\right)^{n}$. 


\section{Capítulo 1}

\section{Propriedades Gerais do Modelo}

\subsection{Introdução}

Neste capítulo vamos apresentar uma visão geral do modelo em questão, constituido de campos escalares carregados não massivos com auto-interação sêxtupla, $g\left(\phi^{*} \phi\right)^{6}$, acoplados minimamente com um campo de gauge abeliano $A_{\mu}$ em três dimensōes, ao qual adicionaremos o termo topológico de Chern-Simons(CS). Analisaremos as simetrias de paridade, inversão temporal e transformações de gauge. Faremos um exame das estruturas ultravioletas e infravermelha da teoria, e apresentaremos as regras de Feynman para a teoria livre. Concluiremos o capítulo com o estudo da equação do grupo de renormalização.

\subsection{Apresentação do Modelo}

O modelo considerado consiste, como já dissemos, de campos escalares $\phi$ carregados, não massivos, com auto-interação sêxtupla, minimamente acoplados a um campo vetorial (CS) $A_{\mu}=\left(A_{0},-\vec{A}\right)$, cuja dinâmica é definida pela densidade de Lagrangiana

$$
\mathcal{L}=\left(D_{\mu} \phi\right)^{*}\left(D^{\mu} \phi\right)-\frac{g}{(3 !)^{2}}\left(\phi^{*} \phi\right)^{3}+-\frac{1}{2 \lambda}\left(\partial_{\mu} A^{\mu}\right)^{2}+\mathcal{L}_{C S}
$$

na qual

$$
\mathcal{L}_{C S}=\frac{1}{2} \varepsilon^{\mu \nu \lambda} A_{\mu l} \partial_{\nu} A_{\lambda}
$$


Em (1.1), $\mathcal{L}_{C S}$ é o Termo de Chern-Simons, $D_{\mu}=\partial_{\mu}-i e A_{\mu}$ é a derivada covariante, $\varepsilon^{\mu \nu \lambda}$ é o tensor anti-simétrico de Levi-Civita normalizado, cujo sinal é fixado por $\varepsilon^{012}=+1$. Adotaremos ainda o sistema de unidades naturais, $\hbar=c=1$, e a métrica de Minkowski é fixada de maneira que $g^{\mu \nu}=\operatorname{Diag}(1,-1,-1)$. O último termo de (1.1) é o termo de fixação de gauge que, como veremos ao discutir o propagador do campo $A_{\mu}$, será considerado, por simplicidade, no limite de Landau, ou seja, $\lambda \rightarrow 0$. Sem a inclusão do termo de fixação de gauge (isto é, $\lambda \rightarrow 0$ ) a equação de movimento para $A_{\mu}$ pode ser obtida em termos dos campos de matéria, representando, desta forma, apenas um vínculo imposto sobre o sistema e implicando na inexistência de dinâmica para o campo $A_{\mu}[27]$. Como neste trabalho desejamos tratar $A_{\mu}$ como um campo propagante o termo de fixação de gauge foi incluído. Temos ainda, concluindo a descrição de (1.1), que $g$ é a constante de acoplamento da interação sêxtupla, e $\alpha$ é o parâmetro de CS.

Uma vez que a ação, $S=\int d^{d} x \mathcal{L}$, é adimensional as quantidades apresentadas em (1.1) possuem, em $d$ dimensões genéricas, as seguintes dimensões de massa

$$
\begin{aligned}
{[\phi] } & =M^{\frac{d-2}{2}} \\
{\left[A_{\mu}\right] } & =M^{\frac{d-1}{2}} \\
{[g] } & =M^{-2(d-3)} \\
{[e] } & =M^{\frac{3-d}{2}}
\end{aligned}
$$

ou, para o caso específico deste trabalho em que $d=3$, temos que $[\phi]=1 / 2,\left[A_{\mu}\right]=1 \mathrm{e}$ as demais grandezas, $g$ e $e$, são adimensionais.

\subsection{Regras de Feynman para a Teoria Livre}

A função de Green de dois pontos para o campo $A_{\mu}, D_{\mu \nu}=<0\left|\hat{T} A_{\mu}(x) A_{\nu}(y)\right| 0>$, na qual $\hat{T}$ é o operador ordenação temporal, corresponde ao propagador livre para o campo $A_{\mu}$, $D_{\mu \nu}$. Para obtê-lo devemos aplicar a equação de Euler-Lagrange para o campo $A_{\mu}(x)$ em (1.1). Assim fazendo obtemos a equação de movimento para $A_{\mu}$, dada por[28][29]

$$
\frac{1}{4 \pi \alpha} \varepsilon^{\mu \nu \lambda} \partial_{\nu} A_{\lambda}+\frac{1}{\lambda} \partial^{\mu}\left(\partial^{\lambda} A_{\lambda}\right)=j^{\mu}
$$

sendo

$$
j^{\mu}=i e\left[\phi^{*}\left(\partial^{\mu} \dot{\phi}\right)-\left(\partial^{\mu} \phi^{*}\right) \phi\right]-2 e^{2} \dot{\phi}^{*} \phi A^{\mu}
$$

Considerando que a fonte $j^{\mu}$ é pontual e localizada na origem de maneira que $D^{\mu \nu}(x)$ seja solução da equação

$$
\left[\frac{1}{4 \pi \alpha} \varepsilon^{\mu \nu \lambda} \partial_{\nu}+\frac{1}{\lambda} \partial^{\mu} \partial^{\lambda}\right] D_{\mu \alpha}(x-y)=i \delta_{\alpha}^{\lambda} \delta^{(3)}(x-y),
$$


e usando nesta a representação de Fourier para $D_{\mu \alpha}(x-y)$ e $\delta^{(3)}(x-y)$

$$
D_{\mu \alpha}(x-y)=\frac{1}{(2 \pi)^{3}} \int d^{d} k e^{-i k(x-y)} D_{\mu \alpha}(k)
$$

e

$$
\delta^{(3)}(x-y)=\frac{1}{(2 \pi)^{3}} \int d^{d} k e^{-i k(x-y)},
$$

podemos escreve-la no espaço dos momentos como

$$
\left[\frac{1}{4 \pi} \varepsilon^{\mu \nu \lambda} k_{\nu}+\frac{1}{\lambda} k^{\mu} k^{\lambda}\right] D_{\mu \alpha}(k)=-i \delta_{\alpha}^{\lambda}
$$

A forma mais geral possível que o tensor de segunda ordem $D^{\mu \alpha}$ pode assumir é $D^{\mu \alpha}=a\left(k^{2}\right) g^{\mu \alpha}+b\left(k^{2}\right) k^{\mu} k^{\alpha}+c\left(k^{2}\right) \varepsilon^{\mu \alpha \beta} k_{\beta}$. Substituindo esta forma geral em (1.8), arrumando convenientemente cada termo e obtendo os coeficientes $a\left(k^{2}\right), b\left(k^{2}\right)$ e $c\left(k^{2}\right)$, vê-se que o propagador do campo $A_{\mu}$ será

$$
D^{\mu \nu}(k)=-i \lambda \frac{k^{\mu} k^{\nu}}{k^{4}}+2 \pi \varepsilon^{\mu \nu \lambda} \frac{k_{\lambda}}{k^{2}} .
$$

A escolha do gauge de Landau, $\lambda \rightarrow 0$, nos leva um melhor comportamento infravermelho das funções de Green[30][31]. Com esta escolha a equação acima toma a forma

$$
D^{\mu \nu}(k)=2 \pi \varepsilon^{\mu \nu \lambda} \frac{k_{\lambda}}{k^{2}}
$$

O propagador livre para o campo escalar $\phi, \Delta_{0}(x-y)=<0\left|\hat{T} \phi(x) \phi^{*}(y)\right| 0>$ é obtido de forma semelhante, via a obtenção da equação de movimento do campo $\phi$ e sua passagem para o espaço dos momentos. Assim fazendo obtemos que

$$
\Delta_{0}(p)=\frac{i}{p^{2}} .
$$

Escrevendo explicitamente a derivada covariante $D_{\mu}=\partial_{\mu}-i e A_{\mu}$ em (1.1) vemos que a Lagrangiana de interação é dada por

$$
\mathcal{L}_{i n t}=-i e A_{\mu}\left[\phi^{*}\left(\partial^{\mu} \phi\right)-\left(\partial^{\mu} \dot{\phi}^{*}\right) \phi\right]+e^{2} A^{\mu} A_{\mu} \phi^{*} \dot{\phi}-\frac{g}{(3 !)^{2}}\left(\dot{\phi}^{*} \phi\right)^{3}
$$

Esta Lagrangiana de interação pode ser escrita de forma a manter a adimensionalidade da ação em $d$ dimensões. Com base nas duas últimas expressões do conjunto de igualdades 
mostrado em (1.2) vemos que a adimensionalidade pode ser mantida se as grandezas $g \mathrm{e}$ $e$, adimensionais em 3 dimensões, forem multiplicadas, respectivamente, por um fator de massa da forma $\mu^{2 \epsilon}$ e $\mu^{\frac{\epsilon}{2}}$. sendo $\epsilon=3-d$. Portanto podemos escrever (1.12) como

$$
\mathcal{L}_{i n t}=-i e \mu^{\frac{\epsilon}{2}} A_{\mu}\left[\phi^{*}\left(\partial^{\mu} \dot{\phi}\right)-\left(\partial^{\mu} \dot{\phi}^{*}\right) \phi\right]+e^{2} \mu^{\epsilon} A^{\mu} A_{\mu} \phi^{*} \phi-\frac{g \mu^{2 \epsilon}}{(3 !)^{2}}\left(\phi^{*} \phi\right)^{3} .
$$

Como já dissemos $\mu$ é um parâmetro de massa arbitrário, chamado de ponto de renormalização . Esta construção é útil quando no cálculo dos diagramas de Feynman é usada a regularização dimensional ( que será discutida por nós oportunamente), na qual para efeito de integração, a dimensão do espaço-tempo é extendida para uma dimensão genérica $d=3-\epsilon$.

Segundo as equações (1.10), (1.11) e baseados em (1.13) podemos escrever as regras de Feynman, e suas respectivas representações gráficas, como segue:

\section{a) Propagadores}

Propagador do campo $\phi$

$$
\Delta_{0}(p)=\longrightarrow \frac{i}{p^{2}}
$$

Propagador do campo $A_{\mu}$

$$
D^{\mu \nu}(k)=\text { vommmmmanmmon } \mu=\varepsilon^{\mu \nu \lambda} \frac{k_{\lambda}}{k^{2}} ;
$$

b) Vértices

$$
\Gamma^{\mu}=\sum_{p_{1}}^{\frac{\xi}{k}}=-i e\left(p_{1}+p_{2}\right)^{\mu} \mu^{\frac{\varepsilon}{2}}
$$



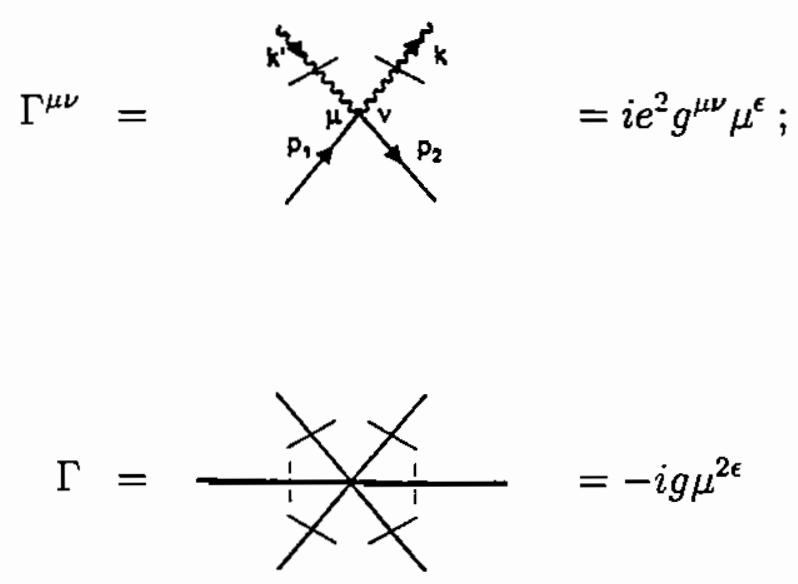

Adicionalmente deve-se considerar que: 1) cada vértice carrega uma delta de conservação de momento, 2) cada circuito fechado de momento ("loop") tem um tri-momento confinado no circuito e deve ser integrado com a medida $d^{3} k /(2 \pi)^{3}$, e 3 ) cada gráfico tem um fator combinatorial multiplicativo, devido as várias maneiras de arranjar os propagadores internos segundo a configuração dos propagadores externos, dividido pelo fatorial do número de vértices.

\subsection{O Grau de Divergência Superficial}

É sabido que a integral sobre os circuitos fechados de momentos internos em um diagrama de Feynman pode fornecer resultados divergentes. Antes de nos determos no tratamento destas divergências é útil saber identificá-las em cada diagrama específico. Isto é feito atrarés do grau de divergência superficial que, para um diagrama genérico $\gamma$ é dado por[32][33!

$$
d(\gamma)=d L-n_{-A}-2 n_{B}+\sum_{a} D_{a}
$$

na qual

$$
\begin{aligned}
& d=\text { dimensão do espaço-tempo } \\
& L=\text { número de "loops" } \\
& n_{-}=\text {número de linhas internas de } A_{\mu} \\
& n_{B}=\text { número de linhas internas de } \phi \\
& D_{a}=\text { número de derivadas do vértice de interação } .
\end{aligned}
$$

A equaçào (1.19) nos informa com que potência de momento o diagrama $\gamma$ diverge (se esse for o caso). Para simplificar a análise de $d(\gamma)$ vamos obter uma expressão para ele de forma que dependa apenas da quantidade de cada tipo de vértice e da natureza 
dos campos externos (já que a idéia da teoria de perturbação é acrescentar vértices, interações, em ordens cada vez maiores). Para tanto, vamos observar que é possível obter uma relação entre o número de "loops" (ou o número de momentos independentes que devem ser integrados) e o número de linhas internas e vértices. Sabemos que existe uma delta de conservação de momento em cada um dos $V$ vértices, uma delta de conservação do momento total do diagrama, e uma integral para cada linha. Assim, obteremos $(V-1)$ relações entre os momentos que podem ser usados para efetuar $(V-1)$ integrais, restanto portanto $n_{A}+n_{B}-V+1$ momentos independentes, ou seja,

$$
L=n_{A}+n_{B}-V+1
$$

Chamando ainda de $v_{a}$ o número total de linhas chegando em cada vértice e lembrando que cada linha interna é contada duas vezes, temos as relações

$$
\begin{aligned}
& \sum_{a} v_{a}^{A}=N_{A}+2 n_{A} \\
& \sum_{a} v_{a}^{B}=N_{B}+2 n_{B}
\end{aligned}
$$

na qual

$N_{A}=$ número de linhas externas de $A_{\mu}$,

$N_{B}=$ número de linhas externas de $\phi$,

$v_{a}^{A}=$ número de linhas do campo $A_{\mu}$ chegando no vértice $V_{a}$,

$v_{a}^{B}=$ número de linhas do campo $\phi$ chegando no vértice $V_{a}$,

$\sum_{a}=$ somatória sobre os vértices.

Usando (1.19), (1.20) e (1.21), temos que

$$
d(\gamma)=d-\left(\frac{d-1}{2}\right) N_{A}-\left(\frac{d-2}{2}\right) N_{B}-\sum_{a}\left[d-d_{a}\right]
$$

sendo $d_{a}$ a dimensão do vértice

$$
d_{a}=D_{a}+\left(\frac{d-1}{2}\right) v_{a}^{A}+\left(\frac{d-2}{2}\right) v_{a}^{B} .
$$

Segundo o valor de (1.23), o vértice $V_{a}^{r}$ pode ser classificado como super-renormalizável, renormalizável ou não renormalizável se temos, respectivamente $d_{a}\left\langle d, d_{a}=d\right.$ ou $\left.d_{a}\right\rangle$ $d$. Assim, a presença de vértices super-renormalizáveis melhora o comportamento ultravioleta da teoria, acontecendo o inverso quando temos vértices não renormalizáveis. Se a teoria contiver somente vértices super-renormalizáveis, ela é dita super-renormalizável; se a teoria contiver pelo menos um vértice não renormalizável é dita não renormalizável; 
e se a teoria não contiver nenhum vértice não renormalizável e pelo menos um vértice renormalizável, é dita renormalizável.

Para o nosso caso, segundo (1.23), vemos que todos os vértices da teoria, dados por (1.16), (1.17) e (1.18) possuem $d_{a}=3$. O grau de divergência superficial do modelo tratado aqui, em três dimensões, fica

$$
d(\gamma)=3-N_{A}-\frac{1}{2} N_{B}
$$

Observe que, neste caso, $d(\gamma)$ não depende do número de vértices $v_{a}^{A}$ ou $v_{a}^{B}$. Isto é, a divergência das funções de Green com $N_{A}$ e $N_{B}$ fixos não cresce com a ordem de perturbação . Além disso, somente um número finito de processos físicos (definidos por $N_{A}$ e $N_{B}$ ) são divergentes. A nossa teoria é dita renormalizável.

Como veremos brevemente, nos restrigiremos neste trabalho basicamente as funções de dois e seis pontos do campo $\phi$. Por (1.24) vemos que as funções de dois pontos são quadraticamente divergentes, enquanto que as funções de seis pontos são logaritimicamente divergentes.

\subsection{Transformações de Simetria}

Vamos investigar nesta seção as transformações discretas de paridade, inversão temporal, conjugação de carga e as transformações de "gauge".

A transformação de paridade (P) é um processo discreto que, em duas dimensões espaciais, consiste na inversão de um dos eixos coordenados (a inversão de ambos os eixos equivaleria a uma rotação). Sendo $x_{1}$ o eixo invertido espacialmente, temos

$$
\left(x_{0}, x_{1}, x_{2}\right) \rightarrow\left(x_{0},-x_{1}, x_{2}\right)
$$

e consequentemente

$$
\left(\partial_{0}, \partial_{1}, \partial_{2}\right) \rightarrow\left(\partial_{0},-\partial_{1}, \partial_{2}\right)
$$

Chamando de $\hat{\mathcal{P}}$ o operador unitário $\left(\hat{\mathcal{P}}^{-1} \hat{\mathcal{P}}=1\right)$, que implementa tal mudança, podemos escrever que os campos escalares e as componentes do campo de gauge são transformadas como

$$
\begin{aligned}
\hat{\mathcal{P}} \phi\left(x_{0}, x_{1}, x_{2}\right) \hat{\mathcal{P}}^{-1} & =\phi\left(x_{0},-x_{1}, x_{2}\right) \\
\hat{\mathcal{P}} A^{0}\left(x_{0}, x_{1}, x_{2}\right) \hat{\mathcal{P}}^{-1} & =A^{0}\left(x_{0},-x_{1}, x_{2}\right) \\
\hat{\mathcal{P}} A^{1}\left(x_{0}, x_{1}, x_{2}\right) \hat{\mathcal{P}}^{-1} & =-A^{1}\left(x_{0},-x_{1}, x_{2}\right) \\
\hat{\mathcal{P}} A^{2}\left(x_{0}, x_{1}, x_{2}\right) \hat{\mathcal{P}}^{-1} & =A^{2}\left(x_{0}:-x_{1}, x_{2}\right)
\end{aligned}
$$


de maneira que as equações de movimento dos campos livres e a Lagrangiana livre não sejam afetados pela reflexão espacial (conforme requer a teoria clássica).

Segundo o mesmo critério podemos definir a transformação de inversão temporal $T^{\mathbf{l}}$. A transformação de inversão temporal é também um processo discreto que, mantendo fixas as coordenadas espaciais, inverte o sinal da coordenada temporal. Segundo esta definição, temos

$$
\left(x_{0}, x_{1}, x_{2}\right) \rightarrow\left(-x_{0}, x_{1}, x_{2}\right)
$$

e ainda

$$
\left(\partial_{0}, \partial_{1}, \partial_{2}\right) \rightarrow\left(-\partial_{0}, \partial_{1}, \partial_{2}\right) .
$$

Chamando de $\hat{\mathcal{T}}$ o operador que implementa tal mudança, podemos escrever que os campos escalares e as componentes do campo de gauge são transformadas como

$$
\begin{aligned}
\hat{\mathcal{T}} \phi\left(x_{0}, x_{1}, x_{2}\right) \hat{\mathcal{T}}^{-1} & =\phi^{*}\left(-x_{0}, x_{1}, x_{2}\right) \\
\hat{\mathcal{T}} A^{0}\left(x_{0}, x_{1}, x_{2}\right) \hat{\mathcal{T}}^{-1} & =A^{0}\left(-x_{0}, x_{1}, x_{2}\right) \\
\hat{\mathcal{T}} A^{i}\left(x_{0}, x_{1}, x_{2}\right) \hat{\mathcal{T}}^{-1} & =-A^{i}\left(-x_{0}, x_{1}, x_{2}\right)
\end{aligned}
$$

sendo que $i=1,2$ na última igualdade acima representa as componentes espaciais de $A_{\mu}$.

Percebe-se que diante das transformações de paridade e inversão temporal, todos os termos da Lagrangiana (1.1), exceto o termo de Chern-Simons, são invariantes[34]. Ou seja, o termo de Chern-Simons quebra explicitamente a simetria $\hat{\mathcal{P}}$ e $\hat{\mathcal{T}}$ na Lagrangiana

$$
\begin{aligned}
& \hat{\mathcal{P}} \mathcal{L}_{C S} \hat{\mathcal{P}}^{-1}=-\mathcal{L}_{C S} \\
& \hat{\mathcal{T}} \mathcal{L}_{C S} \hat{\mathcal{T}}^{-1}=-\mathcal{L}_{C S} .
\end{aligned}
$$

Cabe observar ainda que a Lagrangiana inteira, ou seja, incluindo o termo de ChernSimons, é invariante quando as transformações $\hat{\mathcal{P}}$ e $\hat{\mathcal{T}}$ são efetuadas conjuntamente.

A conjugação de carga $(C)$ é uma transformação discreta que não atua nas coordenadas espaço-temporais. Ela age de forma a substituir uma partícula por uma anti-partícula correspondente e vice-versa. Sendo $\hat{\mathcal{C}}$ o operador que implementa esta transformação , podemos escrever que os campos escalares e o campo de "gauge" transformam-se da forma[35]

$$
\begin{aligned}
\hat{\mathcal{C}} \phi\left(x_{0}, x_{1}, x_{2}\right) \hat{\mathcal{C}}^{-1} & =\phi^{*}\left(x_{0}, x_{1}, x_{2}\right) \\
\hat{\mathcal{C}} \dot{\phi}^{*}\left(x_{0}, x_{1}, x_{2}\right) \hat{\mathcal{C}}^{-1} & =\phi\left(x_{0}, x_{1}, x_{2}\right) \\
\hat{\mathcal{C}} A^{i}\left(x_{0}, x_{1}, x_{2}\right) \hat{\mathcal{C}}^{-1} & =A^{\mu}\left(x_{0}, x_{1}, x_{2}\right)
\end{aligned}
$$

\footnotetext{
${ }^{1}$ A transformação de inversão temporal difere da transformação espacial tanto do ponto de vista físico quanto matemático. Nela as configurações iniciais e finais dos campos são permutadas e $\hat{\mathcal{T}}$ deve ser tido como sendo um operador anti-hermitiano de maneira a manter a positividade do espectro de energia.
} 
e percebe-se que a Lagrangiana (1.1) é invariante sob esta transformação .

Diante desta última conclusão, e daquelas obtidas para a transformações $\mathrm{P}$ e $\mathrm{T}$, vê-se que a teoria descrita por (1.1) possue invariância CPT.

Por fim, além da invariância CPT, vê-se que perante uma transformação de "gauge" local da forma

$$
\begin{aligned}
A_{\mu} \rightarrow A_{\mu}^{\prime} & =A_{\mu}+\frac{1}{e} \partial_{\mu} \Delta(x) \\
\phi \rightarrow \phi^{\prime} & =e^{-i \Delta(x)} \phi
\end{aligned}
$$

a Lagrangiana (1.1) muda apenas por uma derivada total

$$
\mathcal{L}(x) \rightarrow \mathcal{L}^{\prime}(x)=\mathcal{L}(x)+\partial_{\lambda}\left(\frac{1}{2} \varepsilon^{\lambda \mu \nu} A_{\mu} \partial_{\nu} \Delta\right)
$$

mantendo, portanto, a ação clássica invariante.

\subsection{Renormalização do Modelo}

O grau de divergência superficial $d(\gamma)$ discutido por nós na seção 1.3 está relacionado com o comportamento assintótico da teoria no ultravioleta e. segundo os critérios determinados quando analisamos $d(\gamma)$, a teoria abordada por nós é renormalizável (a teoria apresenta apenas vértices renormalizáveis). O procedimento geral da renormalização consiste em mostrar em cada ordem de perturbação, que as grandezas básicas do modelo (constantes de acoplamentos e funções de Green, por exemplo) podem ser escritas em termos de valores finitos, através do cancelamento das divergências pela introdução de contratermos na Lagrangiana.

Ao representarmos uma amplitude de Feynman podemos. em geral, escrevê-la como sendo a soma de duas partes, uma finita e outra infinita. Porém, dependendo da forma que explicitamos esta última parte, percebemos que a primeira (a parte finita) é afetada, nos levando a constatar alguma ambiguidade em sua definição. Assim, além da densidade de Lagrangiana, é necessário, para predizermos quantidades físicas, especificar a maneira pela qual estas quantidades finitas são definidas. Esta especificação consiste em escolher um método que regule as integrais de Feynman: isolando os infinitos dos diagramas, e retire estas divergências sistematicamente.

A escolha do método de regularização e renormalização é uma questão de conveniência. Aqui vamos usar a regularização dimensional[37] e renormalização por "subtração minimal" dos infinitos[38][39][40].

Neste método, concebe-se que, para efeito de cálculo, as integrais são efetuadas em um dimensão espaço-temporal $d$ genérica, porém suficientemente pequena de maneira a fazer convergir as amplitudes. Na continuaçāo analítica das integrais de Feynman para $d$ 
aproximando-se da dimensão original (para o nosso caso, $d=3-\epsilon, \epsilon \rightarrow 0$ ), na qual as divergências estão presentes, as singularidades das integrais são explicitadas através dos p'olos de funções Gama da forma $(1 / \epsilon)^{n}$, com $n$ dependendo da ordem de perturbação . A regularização dimensional preserva todas as propriedades da teoria que independem da dimensão do espaço-tempo, como as identidades de Ward-Takahashi, sendo desta forma uma das regularizações mais convenientes para as teorias que envolvem campos de "gauge" (métodos com "cut-off" nos momentos destroem a invariância de "gauge"). Outra caracteristica que torna a regularização dimensional especialmente útil é ela não apresentar as ambiguidades associadas com a distribuição do fluxo dos momentos nos diagramas de Feynman, como sucede na regularização via "cut-off" [41].

Uma peculariedade da regularização dimensional que também vale ser lembrada é aquela que diz respeito às integrais de um "loop"quando a dimensão original do espaçotempo é impar. Como já dissemos, as divergências são explicitadas através dos pólos da função Gama, mais propriamente quando o argumento da função é um inteiro negativo ou nulo. Se olharmos para a fórmula geral que representa uma amplitude na ordem de 1-"loop",

$$
\int \frac{d^{d} k}{(2 \pi)^{d}} \frac{\left(k^{2}\right)^{a}}{\left(k^{2}+m^{2}\right)^{b}}=\frac{1}{(4 \pi)^{(d / 2)}}\left(m^{2}\right)^{\frac{d}{2}+a-b} \frac{\Gamma\left(\frac{d}{2}+a\right) \Gamma\left(b-a-\frac{d}{2}\right)}{\Gamma\left(\frac{d}{2}\right) \Gamma(b)}
$$

vemos que nenhum pólo da função Gama ocorre para $d$ impar e quaisquer valores inteiros de $a$ e $b[42][22]$. Assim as integrais de Feynman em 1- "loop", que são formalmente divergentes por contagem de potências, tornam-se finitas pela ausência de pólos da função Gama. Veremos que ao usarmos a regularização dimensional apenas os diagramas com um número par de "loops"são efetivamente divergentes.

No próximo capítulo, no qual calculamos as amplitudes de Feynman, voltaremos ao método da regularização dimensional, e discutiremos os aspectos práticos da sua implementação .

\subsubsection{O Método de Contratermos}

Quando se calcula as amplitudes de Feynman a partir de (1.1), vê-se que as funções de Green obtidas destes cálculo são divergentes.

O método de contratermos consiste basicamente em adicionar novos termos à densidade de Lagrangiana com o intuito de subtrair a parte divergente das funções de Green. Sendo a teoria renormalizável, é suficiente um número finito de contratermos para torná-la finita em qualquer ordem de perturbação, e estes contratermos são da mesma forma daqueles monômios originalmente presentes na Lagrangiana. Este é o caso do nosso modelo no qual a divergência superficial dos gráficos de Feynman, (1.24), mostra que somente os diagramas com $\left(N_{A}, N_{B}\right)=(2,0),(0,2),(0,4)$ e $(2,2),(0,6)$ são divergentes. 
Vamos iniciar o processo de renormalização substituindo a densidade de Lagrangiana (1.1) pela densidade de Lagrangiana renormalizada, funcionalmente semalhante à (1.1) mas com parâmetros e campos não renormalizados que diferem dos parâmetros e campos originais por fatores infinitos. Ou seja, vamos escrever

$$
\begin{aligned}
\mathcal{L}_{r} & =\left(\partial_{\mu} \phi_{0}\right)^{*}\left(\partial^{\mu} \phi_{0}\right)-i e_{0} A_{0 \mu}\left[\phi_{0}^{*}\left(\partial^{\mu} \phi_{0}\right)-\left(\partial^{\mu} \phi_{0}^{*}\right) \phi_{0}\right]+e_{0}^{2} A_{0}^{\mu} A_{0 \mu} \phi_{0}^{*} \phi_{0}+ \\
& -\frac{g_{0}}{(3 !)^{2}}\left(\phi_{0}^{*} \phi_{0}\right)^{3}+\frac{1}{4 \pi} \varepsilon^{\mu \nu \lambda} A_{0 \mu} \partial_{\nu} A_{0 \lambda},
\end{aligned}
$$

na qual incluindo um índice "zero", em $\phi_{0}, A_{0 \mu}, e_{0}$ e $g_{0}$ indicando-nos que a Lagrangiana está escrita em termos de grandezas não renormalizadas, ou 'nuas'. As funções de Green dos campos $A_{0 \mu}$ e $\phi_{0}$ obtidas por meio desta densidade de Lagrangiana são divergentes.

Quando renormalizamos a teoria, obtemos funções de Green finitas para os campos $\phi$ e $A_{\mu}$, ditos agora renormalizados. Estes campos renormalizados são definidos em termos das chamadas constantes de renormalizacão, $Z_{\phi}$ e $Z_{A}$, da seguinte forma

$$
\phi=Z_{\phi}^{-\frac{1}{2}} \phi_{0} \rightarrow \phi_{0}=Z_{\phi}^{\frac{1}{2}} \phi
$$

$\mathrm{e}$

$$
A^{\mu}=Z_{A}^{-\frac{1}{2}} A_{0}^{\mu} \rightarrow A_{0}^{\mu}=Z_{A}^{\frac{1}{2}} A^{\mu} .
$$

Substituindo (1.37) e (1.38) em (1.36) obtemos uma segunda forma de escrever a nossa Lagrangiana, agora em termos dos campos renormalizados e das constantes de renormalização, ou seja

$$
\begin{aligned}
\mathcal{L}_{r} & =Z_{\phi}\left(\partial_{\mu} \phi\right)^{*}\left(\partial^{\mu} \phi\right)-i e_{0} Z_{A}^{\frac{1}{2}} Z_{\phi} A_{\mu}\left[\phi^{*}\left(\partial^{\mu} \phi\right)-\left(\partial^{\mu} \phi^{*}\right) \phi\right]+e_{0}^{2} Z_{\phi} Z_{A} A^{\mu} A_{\mu} \phi^{*} \phi \\
& -\frac{g \mu^{2 \epsilon}}{(3 !)^{2}} Z_{\phi}^{3}\left(\phi^{*} \phi\right)^{3}+\frac{1}{4 \pi} Z_{A} \varepsilon^{\mu \nu \lambda} A_{\mu} \partial_{\nu} A_{\lambda} .
\end{aligned}
$$

A Lagrangiana acima pode ainda ser escrita de uma terceira forma. ainda equivalente, para efeito da teoria de perturbação, dada por

$$
\mathcal{L}_{r}=\mathcal{L}+\mathcal{L}_{C T}
$$

com

$$
\begin{aligned}
\mathcal{L} & =\left(\partial_{\mu} \phi\right)^{*}\left(\partial^{\mu} \phi\right)-i e \mu^{\frac{\epsilon}{2}} A_{\mu}\left[\phi^{*}\left(\partial^{\mu} \phi\right)-\left(\partial^{\mu} \phi^{*}\right) \phi\right]+e^{2} \mu^{\epsilon} A^{\mu} A_{\mu} \phi^{*} \phi \\
& -\frac{g \mu^{2 \epsilon}}{(3 !)^{2}}\left(\phi^{*} \phi\right)^{3}+\frac{1}{4 \pi} \varepsilon^{\mu \nu \lambda} A_{\mu} \partial_{\nu} A_{\lambda}
\end{aligned}
$$




$$
\begin{aligned}
\mathcal{L}_{C T} & =A\left(\partial_{\mu} \phi\right)^{*}\left(\partial^{\mu} \phi\right)-i e \mu^{\frac{\epsilon}{2}} D A_{\mu}\left[\phi^{*}\left(\partial^{\mu} \phi\right)-\left(\partial^{\mu} \phi^{*}\right) \phi\right]+e^{2} \mu^{\epsilon} E A^{\mu} A_{\mu} \phi^{*} \phi \\
& -\frac{g \mu^{2 \epsilon} C}{(3 !)^{2}}\left(\phi^{*} \phi\right)^{3}+\frac{1}{4 \pi} B \varepsilon^{\mu \nu \lambda} A_{\mu} \partial_{\nu} A_{\lambda} .
\end{aligned}
$$

Sendo que $\mathcal{L}$, definida em (1.41), é escolhida de forma que todos os parâmetros nela contidos sejam parâmetros renormalizados. Os contratermos $A, B, C, D$, e $E$ presentes em $\mathcal{L}_{C T}$ são definidos por

$$
\begin{aligned}
A & =\left(Z_{\phi}-1\right) \\
B & =\left(Z_{A}-1\right) \\
g \mu^{2 \epsilon} C & =Z_{0}^{3} g_{0}-g \mu^{2 \epsilon} \\
e \mu^{\frac{\epsilon}{2}} D & =Z_{\phi} Z_{A}^{\frac{1}{2}} e_{0}-e \mu^{\frac{\epsilon}{2}} \\
e^{2} \mu^{\epsilon} E & =Z_{\phi} Z_{A} e_{0}^{2}-e^{2} \mu^{\epsilon},
\end{aligned}
$$

de forma a reproduzir a Lagrangiana (1.39). Nesta última expressão para a Lagrangiana incluímos nos termos de interação os fatores massivos $\mu$, para efeito da regularização dimensional.

Os coeficientes $A, B, C, D$ e $E$ presentes em $\mathcal{L}_{C T}$ são divergentes e devem ser escolhidos convenientemente de maneira a absorver as divergências presentes nas funções de Green quando no limite $\epsilon \rightarrow 0$. Estes contratermos podem ser concebidos como advindos de uma reparametrização de $\mathcal{L}$.

Baseados nas expressões (1.43) até (1.47) podemos dizer que

$$
\begin{aligned}
Z_{\phi} & =1+A \\
Z_{A} & =1+B \\
g_{0} & =g \mu^{2 \epsilon} \frac{(1+C)}{(1+A)^{3}} \\
e_{0} & =e \mu^{\frac{\epsilon}{2}} \frac{(1+D)}{(1+A)(1+B)^{\frac{1}{2}}} \\
e_{0}^{2} & =e^{2} \mu^{\epsilon} \frac{(1+E)}{(1+A)(1+B)}
\end{aligned}
$$

obtendo, portanto, em (1.48) a constante de renormalização da função de onda do campo $\phi$, em (1.49) a constante de renormalização da função de onda do campo $A_{\mu}$, e em (1.50), (1.51) e (1.52) os parâmetros não renormalizados $g_{0}, e_{0}$ e $e_{0}^{2}$; todos escritos em termos dos parâmetros renormalizados e dos contratermos.

A forma da Lagrangiana expressa em (1.40) nos conduz a novas regras de Feynman, dadas por 
a) Propagadores Livres

Propagador do campo $\phi$

$$
\Delta(p)=\longrightarrow
$$

Propagador do campo $A_{\mu}$

$$
D^{\mu \nu}(k)=\text { vammminmmono } \mu=2 \pi \varepsilon^{\mu \nu \lambda} \frac{k_{\lambda}}{k^{2}} ;
$$

b) Vértices

$$
\Gamma_{p^{2}}=\underset{\mathrm{p}}{,}, \frac{1}{\mathrm{p}}=i A p^{2}
$$

$\Gamma^{\mu}=\overbrace{p_{2}}^{\frac{\xi_{k}}{\xi_{1}}}=-i e \mu^{\frac{c}{2}}(1+D)\left(p_{1}+p_{2}\right)^{\mu}$
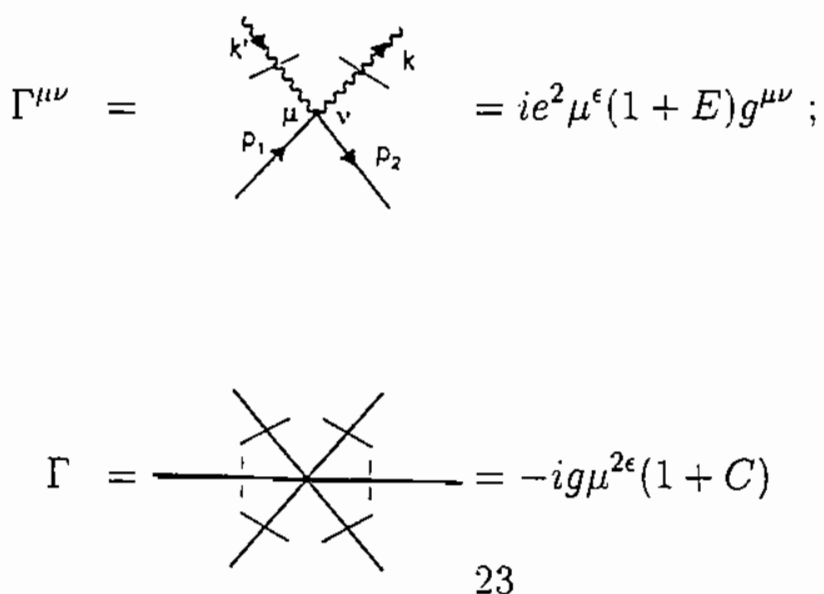
escritas aqui em termos dos parâmetros renormalizados e dos contratermos.

\subsubsection{Relações entre as Constantes de Renormalização}

Algumas propriedades do modelo são preservadas ao implementarmos o esquema de renormalização como, por exemplo, a invariância de "gauge"e, por consequência, as identidades de Ward. Aqui vamos explorar estas propriedades com o objetivo de obter relações entre as constantes de renormalização e reduzir o número de contratermos necessários para tornar a teoria renormalizada.

Uma vez que a regularização dimensional preserva a invariância de "gauge" [37], podemos garantir que as Identidades de Ward (originalmente obtidas para a QED)[43] e sua generalização[44] relacionam as funções de vértice 1-PI (próprias irredutíveis) e os propagadores completos, e são válidas em qualquer ordem de perturbação. Esta relação pode ser explicitada sob a forma

$$
q^{\mu} \Gamma_{\mu}(p, q, p+q)=\Delta^{-1}(p+q)-\Delta^{-1}(p),
$$

ou seja, graficamente representada pela figura (1.1), (1.2) e (1.3)

Tomando em (1.60) o limite $q_{\mu} \rightarrow 0$, podemos ainda obter a identidade de Ward na forma

$$
\frac{\partial \Delta^{-1}(p)}{\partial p^{\mu}}=\Gamma_{\mu}(p, 0, p)
$$

nos dizendo que a diferenciação do inverso do propagador $\Delta(p)$ com relação a $\mu$ corresponde à inserção de um "fóton" (campo de "gauge") de momento zero no propagador de $\phi$. Em princípio as grandezas relacionadas pelas identidades de Ward (funções de vértice e propagadores) sã quantidades divergentes. Estas funçōes admitem uma renormalização multiplicativa, conforme (1.3i) e (1.38). Assim da identidade de Ward para as funçōes renormalizadas segue que

$$
A=D
$$

para identidades de Ward envolvendo funções de vértice de três pontos e

$$
A=E
$$




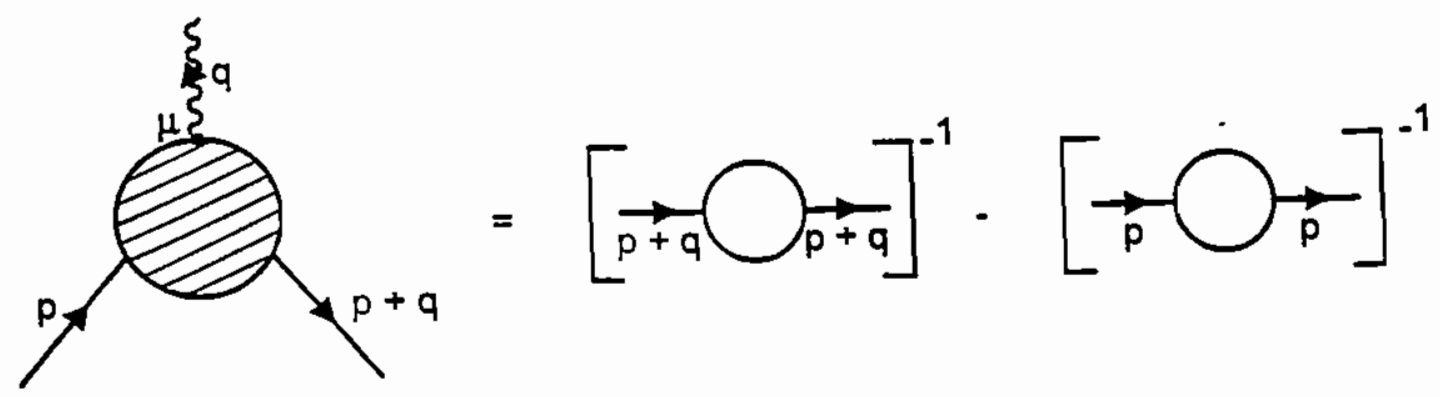

Figura 1.1: Identidade de Ward-Takahashi.
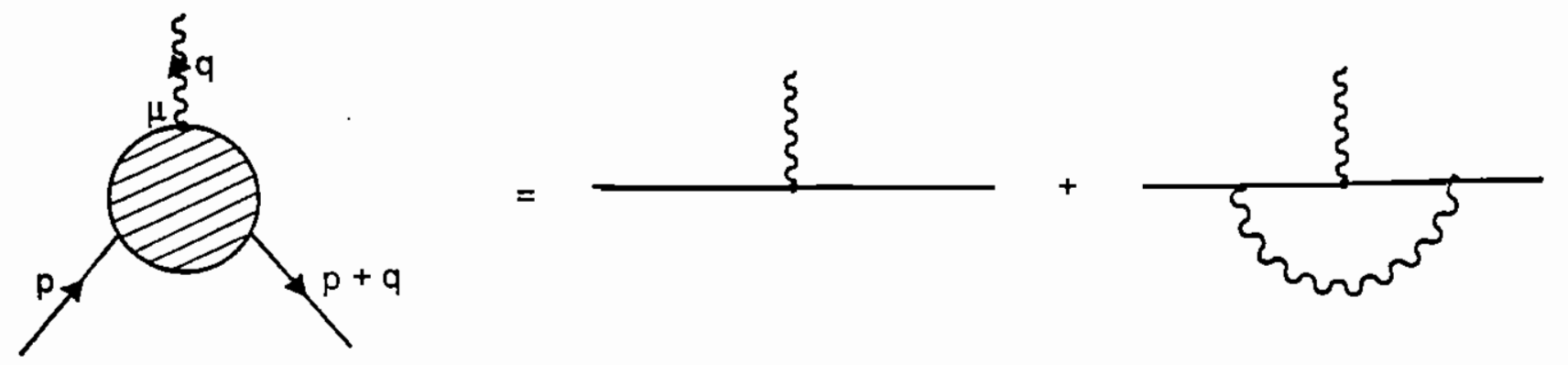

Figura 1.2: Expansão da Função de Vértice $\Gamma_{\mu}(p, q, p+q)$.
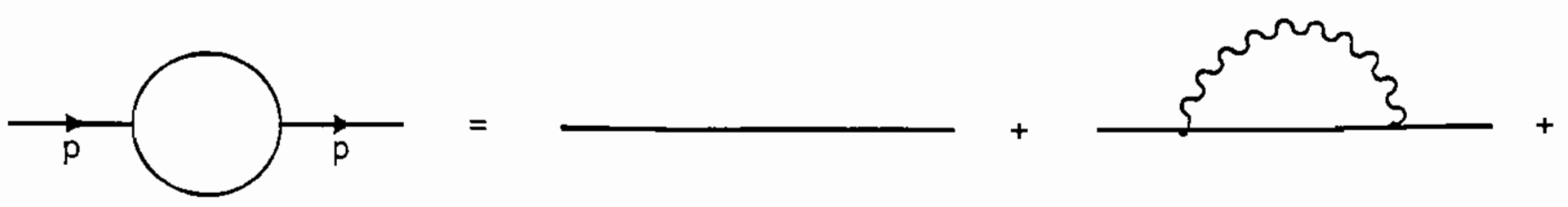

Figura 1.3: Expansão do Propagador $\lambda(p)$ do campo $\phi$. 
para identidades de Ward envolvendo funções de vértice de quatro pontos. Estas relações estão mostradas explicitamente no apêndice E.

Diante destas duas últimas igualdades, podemos escrever novas relações entre as constantes de renormalização (e as grandezas renormalidadas) e os contratermos. Assim, baseados nas equações (1.48) até (1.52), temos que

$$
\begin{array}{rlrl}
\phi_{0} & =Z_{\phi}^{\frac{1}{2}} \phi & & \operatorname{com} Z_{\phi}=1+A \\
A_{0}^{\mu} & =Z_{A}^{\frac{1}{2}} A^{\mu} & & \operatorname{com} Z_{A}=1+B \\
g_{0} & =g \mu^{2 \epsilon} \frac{(1+C)}{(1+A)^{3}}, & \\
e_{0}^{2} & =e^{2} \mu^{\epsilon} \frac{1}{(1+B)}, &
\end{array}
$$

de maneira que reduzimos para três o número de contratermos necessários para renormalizar a teoria.

\subsection{O Grupo de Renormalização}

Ao implementarmos a técnica de regularização dimensional, que isola as divergências das amplitudes de Feynman, vimos que é necessário introduzir um novo parâmetro $\mu$, com dimensão de massa. Como consequência deste procedimento, os parâmetros renormalizados acabam de ganhar uma dependência deste parâmetro (como podemos constatar nas expressões (1.64) até (1.62)). Esta dependência deve-se a liberdade inerente à maneira que optamos separar as partes finita e infinita nas amplitudes (ou, em outras palavras, na liberdade que existe ao separarmos na Lagrangiana não renormalizada, a parte renormalizada e a parte de contratermos). Esta arbitrariedade não tem consequência física, uma vez que qualquer método de subtração conduz aos mesmos resultados físicos. Porém é necessário especificar a escala de momento $\mu$ na qual a subtração dos infinitos é feita. Comumente o valor do parâmetro $\mu$ (chamado de ponto de subtração ou ponto de renormalização) é fixado na teoria, escolhido de maneira que os parámetros renormalizados coincidem com os valores físicos observados.

A este grupo de transformação, envolvendo os vários valores de $\mu$ que mantém invariantes as funçōes de Green renormalizadas, é que chamamos de grupo de renormalização.

As chamadas Equações do Grupo de Renormalização são baseadas nesta idéia, que a teoria física não depende do ponto de renormalização. Uma mudança no ponto $\mu$ deve ser compensada por mudanças nos parâmetros renormalizados como função da energia. Isto garante que as funções de Green renormalizadas (que são funções dos parâmetros renormalizados, do ponto de renormalização e dos momentos externos) mantenham-se invariantes. 
A idéia apresendada no parágrafo anterior, mais propriamente aquela que se refere aos parâmetros físicos (como os acoplamentos) como sendo funções da energia, é fundamental na construção de uma teoria. É na análise do comportamento desta chamada constante de acoplamento efetiva (ou carga efetiva ou "running") na região assintótica que obtemos informaçōes sobre a pertinência do tratamento perturbativo. A investigação das propriedades assintóticas das cargas efetivas é baseada na análise das equações do grupo de renormalização.

As funções de Green de modelos renormalizáveis, que são tornados finitos pela subtração dos termos de p'olo e cujas amplitudes são dimensionalmente regularizadas, satisfazem um tipo de equação do grupo de renormalização conhecida na literatura como equação de t'Hooft-Weinberg[46]. Com o procedimento de subtração mínima as funções do grupo de renormalização, que definiremos brevemente, são independentes de qualquer parâmetro de massa.

Para obter a equação de t'Hoot-Weinberg vamos representar genericamente as funções de Green renormalizadas e não renormalizadas respectivamente por $\Gamma^{(n, m)}\left(p_{i}, g, \alpha, \mu, \epsilon\right)$ e $\Gamma_{0}^{(n, m)}\left(p_{i}, g_{0}, \alpha_{0}, \epsilon\right)$. Nestas funções de vértices 1PI, representamos o acoplamenteo do campo de "gauge" por $\alpha$. Temos ainda que $n$ representa o número de linhas externas amputadas do campo $\phi, \mathrm{m}$ o número de linhas externas amputadas do campo $A_{\mu}$, e $p_{i}$ os n (ou m) momentos dos campos externos (conforme a presença de $\phi$ ou $A_{\mu}$ ). Assim, podemos escrever que

$$
\Gamma_{0}^{(n, m)}\left(p_{i}, g_{0}, \alpha_{0}, \epsilon\right)=Z_{\phi}^{-\frac{n}{2}} Z_{A}^{-\frac{m}{2}} \Gamma^{(n, m)}\left(p_{i}, g, \alpha, \mu, \epsilon\right),
$$

com $Z_{\phi}^{-\frac{n}{2}}$ e $Z_{A}^{-\frac{m}{2}}$ sendo, respectivamente, os fatores de renormalização para cada linha externa, segundo a natureza do campo, $\phi$ ou $A_{\mu}$.

Mantendo $g_{0}, \alpha_{0}$ e $\epsilon$ fixos $\Gamma_{0}^{(n, m)}$ é independente de $\mu$. Portanto

$$
\begin{aligned}
\mu \frac{d}{d \mu} \Gamma_{0}^{(n, m)}\left(p_{i}, g_{0}, \alpha_{0}, \epsilon\right) & =0=\left(\mu \frac{d}{d \mu} Z_{\phi}^{-\frac{n}{2}}\right) Z_{A}^{-\frac{m}{2}} \Gamma^{(n, m)}\left(p_{i}, g, \alpha, \mu, \epsilon\right) \\
& +Z_{\phi}^{-\frac{n}{2}}\left(\mu \frac{d}{d \mu} Z_{A}^{-\frac{m}{2}}\right) \Gamma^{(n, m)}\left(p_{i}, g, \alpha, \mu, \epsilon\right) \\
& +Z_{\phi}^{-\frac{n}{2}} Z_{A}^{-\frac{m}{2}}\left(\mu \frac{d}{d \mu} \Gamma^{(n, m)}\left(p_{i}, g, \alpha, \mu, \epsilon\right)\right) .
\end{aligned}
$$

Se expressarmos a derivada total em $\Gamma^{(n, m)}$ em termos de derivadas parciais,

$$
\frac{d}{d \mu}=\frac{\partial}{\partial \mu}+\frac{\partial g}{\partial \mu} \frac{\partial}{\partial g}+\frac{\partial \alpha}{\partial \mu} \frac{\partial}{\partial \alpha}
$$

e lembrarmos que as constantes de renormalização $Z_{\phi}$ e $Z_{A}$ são escritas em termos das grandezas não renormalizadas, obtemos a equação do grupo de renormalização,

$$
\left[\mu \frac{\partial}{\partial \mu}+\beta_{g} \frac{\partial}{\partial g}+\beta_{\alpha} \frac{\partial}{\partial \alpha}-n \gamma_{\dot{\phi}}-m \gamma_{A}\right] \Gamma^{(n, m)}\left(p_{i}, g, \alpha, \mu, \epsilon\right)=0
$$


sendo

$$
\begin{aligned}
\beta_{g} & =\mu \frac{\partial g}{\partial \mu} \\
\beta_{\alpha} & =\mu \frac{\partial \alpha}{\partial \mu} \\
\gamma_{\phi} & =\frac{\mu}{2} \frac{\partial}{\partial \mu} \log Z_{\phi} \\
\gamma_{A} & =\frac{\mu}{2} \frac{\partial}{\partial \mu} \log Z_{A} .
\end{aligned}
$$

No apêndice $D$, mostramos explicitamente que, até a ordem de perturbação que consideramos no cálculo das funções de dois e seis pontos do campo $\phi$, a função de onda do campo $A_{\mu}$ é finita. Assim, tomando por base a relação (1.67) podemos escrever que

$$
\alpha_{0}=\alpha \mu^{\epsilon} \text {. }
$$

Aplicando o operador diferencial $\mu \frac{d}{d \mu}$ na igualdade acima obtemos, usando a definição (1.72) que

$$
\beta_{\alpha}=\mu \frac{\partial \alpha}{\partial \mu}=-\epsilon \alpha=0 .
$$

Temos ainda que para $Z_{A}=1(B=0)$. Este resultado está de acordo com o Teorema de Coleman-Hill[47], obtido para campos de gauge não abelianos. Extenções deste teorema podem ser vistas nas referências[48]. Para maiores detalhes, no que se refere ao nosso caso, ver apêndice D. A equação (1.74) nos informa, então que

$$
\gamma_{A}=0 \text {. }
$$

E a equação do grupo de renormalização (1.69) reduz-se simplesmente a

$$
\left[\mu \frac{\partial}{\partial \mu}+\beta_{g} \frac{\partial}{\partial g}-n \gamma_{\phi}\right] \Gamma^{(n)}\left(p_{i}, g, \alpha, \mu, \epsilon\right)=0 .
$$

Sendo $\gamma_{\phi}$ chamada de dimensão anômala do campo básico $\phi$ e $\beta_{g}(g)$ a função beta. Esta última nos informa do comportamento da constante de acoplamento como função da energia. Oportunamente analisaremos suas propriedades. No momento, nos deteremos na tarefa de escrever $\gamma_{\phi}(g, \alpha)$ e $\beta_{g}(g, \alpha)$ em termos dos contratermos.

Podemos expressar o acoplamento $g_{0}$, não renormalizado, como uma série de Laurent cujos coeficientes são escritos em termos dos parâmetros renormalizados,

$$
g_{0}=\mu^{2 \epsilon}\left[g+\sum_{n=1}^{\infty} \frac{g_{n}(g, \alpha)}{\epsilon^{n}}\right]
$$


sendo que $g_{n}(g, \alpha)$ é analítica quando $\epsilon \rightarrow 0$. Aplicando o operador $\mu \frac{d}{d \mu}$ sobre (1.79) e usando que $\mu \frac{\partial \alpha}{\partial \mu}=-\epsilon \alpha$, equação (1.76), temos que

$$
2 \epsilon g+2 g_{1}+\mu \frac{\partial g}{\partial \mu}-\alpha \frac{\partial g_{1}}{\partial \mu}+\sum_{n=1}^{\infty} \frac{1}{\epsilon^{n}}\left[2 g_{n+1}+\frac{\partial g_{n}}{\partial g} \mu \frac{\partial g}{\partial \mu}-\alpha \frac{\partial g_{n+a}}{\partial \alpha}\right]=0 .
$$

A função beta é analítica em $\epsilon \rightarrow 0$. Podemos escrever

$$
\begin{aligned}
\mu \frac{\partial g}{\partial \mu} & =d_{0}+d_{1} \epsilon+\mathcal{O}\left(\epsilon^{2}\right) \\
& =\beta_{g}+d_{1} \epsilon
\end{aligned}
$$

incluindo convenientemente um termo de ordem $\epsilon$. Substituindo (1.81) e (1.80) e coletando os termos de mesma ordem em $\epsilon$ obtemos um conjunto de três igualdades

$$
\begin{aligned}
d_{1} & =-2 g \\
d_{0} & =\beta_{g}=\left(\alpha \frac{\partial}{\partial \alpha}+2 g \frac{\partial}{\partial g}-2\right) g_{1}(g, \alpha), \\
d_{0} \frac{\partial}{\partial g} g_{n}(g, \alpha) & =\left(\alpha \frac{\partial}{\partial \alpha}+2 g \frac{\partial}{\partial g}-2\right) g_{n+1}(g, \alpha) .
\end{aligned}
$$

Vamos supor que a constante de renormalização do campo $\phi, Z_{\phi}$, pode ser também escrita como uma série de Laurent, ou seja,

$$
Z_{\phi}=1+\sum_{n=1}^{\infty} \frac{Z_{n}(g, \alpha)}{\epsilon^{n}}
$$

Da expressão para a dimensão anômala do campo $\phi$, equação (1.73), obtemos que

$$
\mu \frac{\partial Z_{\phi}}{\partial \mu}=2 Z_{\phi} \gamma_{\phi} .
$$

Aplicando o operador $\mu \frac{d}{d \mu}$ em (1.85), usando (1.81) e (1.76), e comparando com a equação (1.86) acima, temos

$$
2 Z_{\phi} \gamma_{\phi}=\sum_{n=1}^{\infty} \frac{1}{\epsilon^{n}}\left[\frac{\partial Z_{n}}{\partial g}\left(d_{0}+d_{1} \epsilon\right)-\frac{\partial Z_{n}}{\partial \alpha} \epsilon \alpha\right] .
$$

Substituindo a expressão para $Z_{\phi}$, equação (1.85), na expressão acima obtemos a relação

$$
2 \gamma_{\phi}-d_{1} \frac{\partial Z_{1}}{\partial g}+\alpha \frac{\partial Z_{1}}{\partial \alpha}+\sum_{n=1}^{\infty} \frac{1}{\epsilon^{n}}\left[2 \gamma_{\phi} Z_{n+1}-d_{0} \frac{\partial Z_{n}}{\partial g}-d_{1} \frac{\partial Z_{n+1}}{\partial g}+\alpha \frac{\partial Z_{n+1}}{\partial \alpha}\right]=0
$$


e novamente coletando os termos de cada ordem em $\epsilon$ obtemos as igualdades

$$
\begin{array}{r}
\gamma_{\phi}=\left(-\frac{\alpha}{2} \frac{\partial}{\partial \alpha}+\frac{d_{1}}{2} \frac{\partial}{\partial g}\right) Z_{1} \\
d_{0} \frac{\partial}{\partial g} Z_{n}=\left(2 \gamma_{\phi}-d_{0}-d_{1} \frac{\partial}{\partial g}+\alpha \frac{\partial}{\partial \alpha}\right) Z_{n+1} .
\end{array}
$$

Substituindo em (1.89) o valor de $d_{1}$, equação (1.82) obtemos que a dimensão anômala do campo $\phi$ tem a forma

$$
\gamma_{\phi}=\left(-\alpha \frac{\partial}{\partial \alpha}-g \frac{\partial}{\partial g}\right) Z_{1}(g, \alpha) .
$$

Tendo em mão esta expressão para $\gamma_{\phi}$, juntamente com a expressão para $\beta_{g}$ (equação (1.83), expliciamente

$$
\beta_{g}=\left(\alpha \frac{\partial}{\partial \alpha}+2 g \frac{\partial}{\partial g}-2\right) g_{1}(g, \alpha)
$$

obtemos as funções do grupo de renormalização em termos dos contratermos. Para perceber isto basta lembrar primeiramente que $Z_{\phi}=1+A$ e comparar com (1.85). Assim fazendo temos que

$$
A=\frac{Z_{1}(g, \alpha)}{\epsilon}+\frac{Z_{2}(g, \alpha)}{\epsilon^{2}}+\ldots,
$$

ou seja, $Z_{1}(g, \alpha)$ em (1.91) é o termo de ordem $\epsilon^{-1}$ do contratermo $A$, que renormaliza a função de onda do campo $\phi$. É bom resaltar aqui que $Z_{1}(g, \alpha)$ também pode ser expresso em uma expansão em série nas constantes de acoplamento de acordo com a expansão perturbativa da função de dois pontos.

Para a identificação de $g_{1}(g, \alpha)$ em (1.92) vamos recorrer à equação (1.66),

$$
g_{0}=g \mu^{2 \epsilon} \frac{(1+C)}{(1+A)^{3}} .
$$

Tanto o contratermo $A$ como o $C$ admitem uma expansão em série de potências nas constantes de acoplamento, ou seja, podemos escrever que

$$
(1+C)=1+C_{1}+C_{2}+\ldots
$$

$\mathrm{e}$

$$
(1+A)=1+A_{1}+A_{2}+\ldots
$$


E a equação acima para $g_{0}$ pode ser escrita como

$$
\begin{gathered}
g_{0}=g \mu^{2 \epsilon}\left[1+\left(C_{1}-3 A_{1}\right)+\left(C_{2}-3 A_{2}-3 C_{1} A_{1}\right)+\right. \\
\left.-\left(3 C_{1} A_{2}+3 C_{2} A_{1}\right)-3 C_{2} A_{2}+\ldots\right]
\end{gathered}
$$

sendo que, comparando com a equação (1.79), vemos que $g_{1}(g, \alpha)$ é o resíduo do p'olo simples da equação (1.94).

Cabe observar que tanto $\gamma_{\phi}$ como $\beta_{g}$ são totalmente determinados pelos resíduos dos pólos simples dos contratermos [49]. No capítulo seguinte vamos explicitar os contratermos necessários para cancelar as divergências das funçōes de dois e seis pontos, inclusive, por completeza, os contratermos que admitem pólos duplos.

Ao analisarmos o grau de divergência superficial para a função de dois e seis pontos, vimos que eles são, respectivamente, quadraticamente divergente e logaritimicamente divergente. Os contratermos induzidos por estas funções são, portanto, proporcionais ao quadrado do momento no primeiro caso e proporcionais a uma constante no segundo. Estes resultados estão de acordo com o Teorema de Weinberg[50], no qual nos diz que os contratermos induzidos pelo processo de renormalização são polinômios nos momentos externos, de grau igual ao da divergência superficial.

No próximo capítulo, calcularemos as funções de vértice de dois e seis pontos, e obteremos os contratermos necessários para absorver as divergências presentes em cada caso. 


\section{Capítulo 2}

\section{O Cálculo das Funções de Dois e Seis Pontos}

\subsection{Introdução}

Como já foi dito na introdução deste trabalho, nosso intuito é analisar a influência do termo de Chern-Simons sobre a renormalizibilidade da teoria. Veremos neste capítulo que para a função de dois pontos tal influência dá-se na ordem 2 de perturbação . mais propriamente em $e^{4}$ (ou, equivalentemente, $\alpha^{2}$, sendo que chamaremos de $\alpha$ o parâmetro de expansão perturbativa referente ao acoplamento de Chern-Simons). Neste trabalho, consideraremos que os acoplamentos $\alpha$ e $g$ tem a mesma ordem de grandeza na teoria de perturbação . Para a análise das divergências da função de seis pontos devemos considerar a expansão até a ordem 3 (menor ordem em que se manifestam as divergências), e é nesta ordem de perturbação que, como veremos, dá-se o aparecimento dos pólos duplos.

Iniciaremos este capítulo definindo formalmente as funções de dois e seis pontos. Passaremos, em seguida, ao cálculo de alguns diagramas úteis, que aparecem como subdiagramas dos demais, com o intuito de simplificar a análise e o cálculo das amplitudes mais complicadas.

Concluiremos o capítulo explicitando as divergências das funções de dois e seis pontos e obtendo os contratermos necessários para renormalizar a teoria até a ordem aqui adotada.

Para facilitar o trabalho de cálculo, vamos, sempre que possível, escrever as integrais nos momentos em uma das três formas gerais

$$
\begin{aligned}
& I_{(a)}=\int d^{d} q \frac{1}{\left[-q^{2}\right]^{\alpha}\left[-(q+p)^{2}\right]^{\beta}} \\
& I_{(b)}^{\mu}=\int d^{d} q \frac{q^{\mu}}{\left[-q^{2}\right]^{\alpha}\left[-(q+p)^{2}\right]^{\beta}}
\end{aligned}
$$




$$
I_{(c)}^{\mu \nu}=\int d^{d} q \frac{q^{\mu} q^{\nu}}{\left[-q^{2}\right]^{\alpha}\left[-(q+p)^{2}\right]^{\beta}} .
$$

que no apêndice $C$ são chamadas respectivamente de $(C .1),(C .2)$ e (C.3). Neste apêndice, as integrais acima são calculadas explicitamente e usaremos diretamente estes resultados no decorrer dos cálculos dos diagramas.

Neste capítulo, os diagramas da função de vértice de seis pontos serão calculados escolhendo-se uma situação em que o momento em cada propagador externo será escolhido como sendo $p($ ou $-p)$. Isto facilita consideravelmente o cálculo dos fatores combinatoriais devidos a cada configuração do fluxo dos momentos.

É oportuno também mencionar algo sobre o uso da regularização dimensional quando está presente o tensor $\varepsilon^{\mu \nu \rho}$. É bem conhecido que a influência da regularização dimensional em objetos que quebram a simetria de paridade, como é o caso do tensor de Levi-Civita, levam a problemas na extensão destes objetos para uma dimensão arbitrária d[33]. Este problema da extensão do tensor $\varepsilon^{\mu \nu \rho}$ para $d$ dimensões tem sido discutido sobre vários aspectos por muitos autores[51]. O uso de diferentes prescrições para efetuar esta extensão podem induzir apenas termos finitos no cálculo dos diagramas[52][53]. Entretanto, como estamos interessados apenas na parcela divergente dos diagramas de Feynman (com o intuito de analisar as prorpriedades das funções do grupo de renormalização ), e como em um "loop" os gráficos são finitos e em dois "loops" apresentam pólos simples, os nossos resultados não são afetados pela forma que tratamos a expansão do tensor $\varepsilon^{\mu \nu \rho}$. Como veremos os diagramas das fuções de dois e seis pontos nos quais os termos de ChernSimons estão presentes foram calculados até a ordem de dois "loops". Diagramas em ordem superiores a dois "loops", que apresentam pólos dúplos(e para os quais, portanto, existe colaboração da parte finita), não contém o termo da Chern-Simons.

\subsection{Definições das Funções de Vértice de Dois e Seis Pontos}

A função de Green conexa 1-PI é definida pela série

$$
G_{c}^{(2)}(p)=\Delta_{0}(p)+\Delta_{0}(p)[-i \Sigma(p)] \Delta_{0}(p)+\Delta_{0}(p)[-i \Sigma(p)] \Delta_{0}[-i \Sigma(p)] \Delta_{0}+\ldots,
$$

escrita em termos do propagador livre $\lrcorner_{0}(p)=i / p^{2}$ do campo $\phi$ e da auto-energia própria $-i \Sigma(p)$, ou seja, da soma de todos os gráficos irredutíveis.

A expressão (2.4) pode ser escrita como o produto do propagador livre por uma série geométrica de razão $-i \Sigma$ e reduzir-se a

$$
G_{c}^{(2)}(p)=\frac{i}{p^{2}-\Sigma(p)}
$$


A função de vértice $\Gamma^{(2)}(p)$, ou função própria de dois pontos, é definida como

$$
\Gamma^{(2)}(p)=-\left[G_{c}^{(2)}(p)\right]^{-1},
$$

que, baseados em (2.5), podemos escrever

$$
\Gamma^{(2)}(p)=i p^{2}-i \Sigma(p) .
$$

Os gráficos que compõem a auto-energia $-i \Sigma$ estão ilustrados no apêndice $A$, sendo que devemos excluir deles os propagadores externos. Por definição $-i \Sigma$ corresponde à amplitude pura, sem os campos externos.

A função própria de seis pontos, ou função de vértice de seis pontos, depende apenas dos diagramas 1-PI, também ilustrados no apêndice A, sendo que os propagadores externos devem também ser excluídos. Assim

$$
\Gamma^{(6)}=\Delta^{(2)}\left(p_{1}\right)^{-1} \ldots \Delta^{(2)}\left(p_{6}\right)^{-1} G_{c}^{(6)}\left(p_{1}, \ldots, p_{6}\right),
$$

sendo que $\Delta^{(2)}\left(p_{1}\right)^{-1}$ tem o efeito de excluir os campos externos e $G_{c}^{(6)}$ é a função de Green de seis pontos.

Observando os diagramas que compõem a função de vértice de seis pontos. vemos que a ordem mais baixa em que estes diagramas exibem divergência e que o termos de ChernSimons está presente é 3 (ou seja, ordem cúbica de $\alpha$ ou $g$, ou o produto do quadrado de uma delas pela outra). Assim a expansão perturbativa da função de seis pontos foi considerada até a ordem 3 .

Para a função de vértice de dois pontos adotamos o mesmo critério, e levamos a expansão até a ordem mais baixa que apresenta divergência envolvendo o termo de ChernSimons. Ordens mais altas de perturbação ofereceriam correções aos valores numéricos, sem portanto alterar a natureza do comportamento das funções do grupo de renormalização .

\section{$2.3 \quad$ Sub-diagramas}

Antes de passarmos para o cálculo dos diagramas que contribuem para as funções de vértice de dois e seis pontos propriamente ditas, vamos calcular alguns diagramas que serão especialmente úteis no cálculo de alguns outros. Estes diagramas são, via de regra, sub-diagramas. e seus resultados servirão para compor as demais amplitudes.

Uma observação importante que deve ser feita é que nestes cálculos, exceto quando o diagrama for nulo, vamos manter todos os fatores de $\epsilon$. Eles devem ser mantidos na expressão pois, ao usarmos estas equações como partes de diagramas maiores, estes fatores são fundamentais pois colaborarão na composição dos pólos da função gama. 


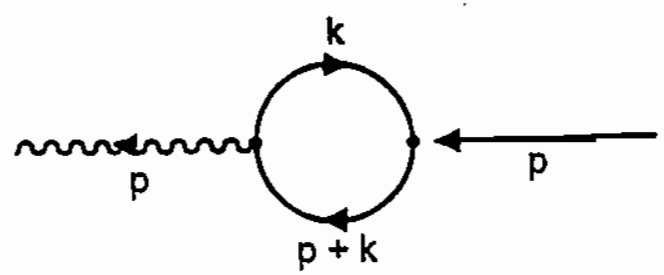

$\Pi_{\alpha \beta}(p)$

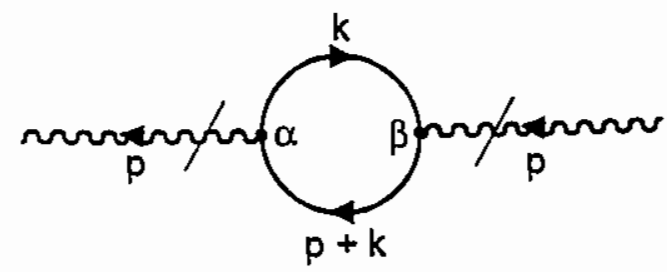

$P_{\mu \nu}(p)$

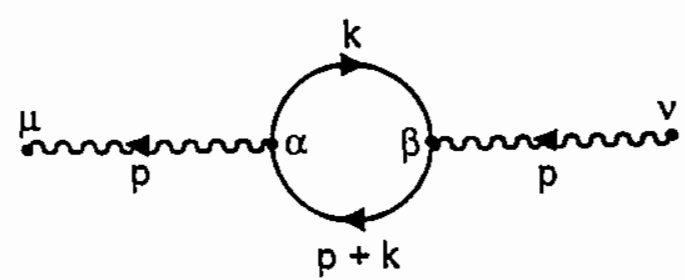

Figura 2.1: Diagramas $\Pi_{\alpha}(p), \Pi_{\alpha \beta}(p)$ e $P_{\mu \nu}(p)$.

Para efeito de regularização dimensional, a medida $\mathcal{D} k$ deve ser tomada por $\mu^{\epsilon} d^{d} k /(2 \pi)^{d}$; com $\epsilon=3-d$. Nesta primeira parte do capítulo, calcularemos as amplitudes sem levar em conta os fatores de vértices (constantes de acoplamentos ou fases imaginárias). Por comodidade. estes fatores serão incluídos posteriormente. assim como os fatores combinatoriais devidos as várias maneiras de contrair os campos internos segundo uma dada configuração dos campos externos.

Vamos calcular inicialmente os diagramas mostrados na figura (2.1)

Segundo a figura (2.1) podemos escrever que

$$
\Pi_{\alpha}(p)=\int \mathcal{D} k(2 k+p)_{\alpha} \frac{i}{(k+p)^{2}} \frac{i}{k^{2}},
$$

ou ainda

$$
\Pi_{\alpha}(p)=-\frac{1}{(2 \pi)^{2}} \int d^{d} k \frac{(2 k+p)_{\alpha}}{\left[-k^{2}\right]\left[-(k+p)^{2}\right]} .
$$

Temos, portanto. duas integrais para serem resolvidas. Estas integrais são da forma apresentadas em (2.2) e 2.3) $\operatorname{com} \alpha=\beta=1$, e cujos resultados são dados. respectivamente, em (C. 4 ) e (C.j). Assin fazendo. temos que

$\Pi_{\alpha}(p)=-\frac{1}{(2 \pi)^{d}} \frac{i \pi^{d / 2} p_{\alpha}}{\left[-p^{2}\right]^{\frac{1}{2}+\frac{c}{2}}}\left[\frac{\Gamma\left(\frac{d}{2}-1\right) \Gamma\left(\frac{d}{2}-1\right) \Gamma\left(2-\frac{d}{2}\right)}{\Gamma(d-1)}-\frac{2 \Gamma\left(\frac{d}{2}\right) \Gamma\left(\frac{d}{2}-1\right) \Gamma\left(2-\frac{d}{2}\right)}{\Gamma(d-1)}\right]$. 
fazendo $d=3$ no termo entre colchetes, lembrando que $\Gamma(1 / 2)=\sqrt{\pi}$ e $\Gamma(3 / 2)=\sqrt{\pi} / 2$, vemos que este termo se anula. Assim

$$
\Pi_{\alpha}(p)=0
$$

Para $\Pi_{\alpha \beta}(p)$, da figura $(2.1)$, nós temos

$$
\Pi_{\alpha \beta}=i^{2} \int \mathcal{D} k \frac{(p+2 k)_{\alpha}(p+2 k)_{3}}{k^{2}(k+p)^{2}}
$$

ainda

$$
\Pi_{\alpha \beta}=-\int \mathcal{D} k \frac{\left[4 k_{\alpha} k_{\beta}+p_{\alpha} p_{\beta}+2 p_{\alpha} k_{\beta}+2 k_{\alpha} p_{\beta}\right]}{k^{2}(k+p)^{2}}
$$

aqui podemos usar novamente o apêndice $C$ para resolver os três tipos de integrais acima. Alternativamente, podemos explicitar os passos do cálculo. Fazendo a parametrização de Feynman da forma mostrada no apêndice B, equação (B.7). chamando $a=(p+k)^{2} \mathrm{e}$ $b=k^{2}$ temos

$$
\Pi_{\alpha \beta}=-\int_{0}^{1} d x \int \mathcal{D} k\left[\frac{\left[4 k_{\alpha} k_{\beta}+p_{\alpha} p_{\beta}+2 p_{\alpha} k_{3}+2 k_{\alpha} p_{\beta}\right]}{\left[-k^{2}-2 k \cdot p x-p^{2} x\right]^{2}}\right] .
$$

Usando agora (B.1), (B.2) e (B.3) para resolver as integrais nós obtemos

$$
\begin{aligned}
\Pi_{\alpha \beta} & =-\frac{i \mu^{\epsilon}}{2^{d} \pi^{\frac{d}{2}}} \Gamma\left(2-\frac{d}{2}\right) \int_{0}^{1} d x\left[\frac{4 p_{\alpha} p_{\beta}}{\left(-p^{2}\right)^{2-\frac{d}{2}}} \frac{x^{2}}{\left(x-x^{2}\right)^{2-\frac{d}{2}}}+\frac{4 g^{\alpha \beta}}{\left(-p^{2}\right)^{2-\frac{d}{2}}} \frac{1}{\left(x-x^{2}\right)^{1-\frac{d}{2}}}\right. \\
& \left.-\frac{4 p_{\alpha} p_{\beta}}{\left(-p^{2}\right)^{2-\frac{d}{2}}} \frac{x}{\left(x-x^{2}\right)^{2-\frac{d}{2}}}+\frac{4 p_{\alpha} p_{\beta}}{\left(-p^{2}\right)^{2-\frac{d}{2}}} \frac{1}{\left(x-x^{2}\right)^{2-\frac{d}{2}}}\right]
\end{aligned}
$$

Os termos que dependem de $x$ no denominador podem ser invertidos, passando para o numerador. Então, usando-se (B.9) para fazer cada integral paramétrica. temos finalmente

$$
\Pi_{\alpha \beta}=-\frac{1}{16 \sqrt{p^{2}}}\left(p_{\alpha} p_{\beta}-p^{2} g_{\alpha \beta}\right)\left(\frac{-4 \pi \mu^{2}}{p^{2}}\right)^{\frac{\varrho}{2}} .
$$

O diagrama $\Pi_{\alpha \beta}$ é chamado de tensor de polarização. Observe que mantivemos explicitamente os fatores de $\epsilon$, sem colapsá-los em $\epsilon \rightarrow 0$.

Para obtermos $P_{\mu \nu}(p)$ na figura (2.1) basta incluir os propagadores de Chern-Simons em $\Pi_{\alpha \beta}$, ou seja,

$$
P_{\mu \nu}(p)=\varepsilon^{\mu \alpha \rho} \frac{p_{\rho}}{p^{2}} \Pi_{\alpha \beta}(p) \varepsilon^{\beta \nu \gamma} \frac{p_{\gamma}}{p^{2}}
$$


Usando as propriedades de anti-simetria do tensor, $\varepsilon^{\mu \alpha \rho} p_{\alpha} p_{\rho}=0$, vê-se que o primeiro termo devido a (2.17) é nulo. Usando em seguida a relação para o produto de dois $\varepsilon^{\mu \alpha \rho}$, equação (B.16) obtemos que

$$
P_{\mu \nu}(p)=\frac{i}{16} \frac{\left(4 \pi \mu^{2}\right)^{\frac{e}{2}}}{\left(-p^{2}\right)^{\frac{3}{2}+\frac{e}{2}}}\left(g_{\mu \nu} p^{2}-p_{\mu} p_{\nu}\right) .
$$

Vamos calcular agora um segundo grupo de diagramas que podem ser visto na figura (2.2).

O diagrama $\Theta(p)$, da figura (2.2) pode ser escrito algebricamente como

$$
\Theta(p)=\int \mathcal{D} k \varepsilon^{\mu \nu \lambda} \frac{k_{\lambda}}{k^{2}} \varepsilon_{\nu \mu \rho} \frac{(k+p)^{\rho}}{(k+p)^{2}},
$$

usando (B.16) a equação acima pode ser escrita como

$$
\Theta(p)=-2 g^{\lambda \rho} \int \mathcal{D} k\left(\frac{k_{\lambda} k_{\rho}}{k^{2}(k+p)^{2}}+\frac{k_{\lambda} p_{\rho}}{k^{2}(k+p)^{2}}\right) .
$$

Na primeira integral, o termo em $k^{2}$ pode ser cancelado no numerador e no denominador, reescrevendo $k+p \rightarrow k^{\prime}$ e usando (B.1) para $p=C=0$, vemos que esta integral é zero. Assim, (2.21) pode ser escrita simplesmente como

$$
\Theta(p)=-\frac{2 g^{\lambda \rho} p_{\rho} \mu^{\epsilon}}{d^{2} \pi^{d}} I_{k}
$$

sendo

$$
I_{k}=\int d^{d} k \frac{k_{\lambda}}{\left[-k^{2}\right]\left[-(k+p)^{2}\right]}=-\frac{i \pi^{3}}{2} p_{\lambda} \frac{\left[-\pi p^{2}\right]^{-\frac{\ell}{2}}}{\left[-p^{2}\right]^{\frac{1}{2}}} .
$$

Substituindo em (2.22) temos para $\Theta(p)$ o resultado

$$
\Theta(p)=-\frac{i}{8} \frac{\left(4 \pi \mu^{2}\right)^{\frac{5}{2}}}{\left[-p^{2}\right]^{-\frac{1}{2}+\frac{\epsilon}{2}}} .
$$

Para o diagrama $\Theta_{\mu}(p)$, figura (2.5), temos

$$
\Theta_{\mu}(p)=\int \mathcal{D} k \varepsilon^{\mu \nu \rho} \frac{k_{\rho}}{k^{2}}(2 p+k)_{\nu} \frac{i}{(p+k)^{2}} .
$$

Pela anti-simetria do $\varepsilon^{\mu \nu \rho}$, o segundo termo da integral acima é nulo, assim $\Theta(p)_{\mu}$ pode ser escrito simplemente como

$$
\Theta_{\mu}(p)=2 i \varepsilon^{\mu \nu \rho} p_{\nu} \int \mathcal{D} k \frac{k_{\rho}}{k^{2}(p+k)^{2}} .
$$



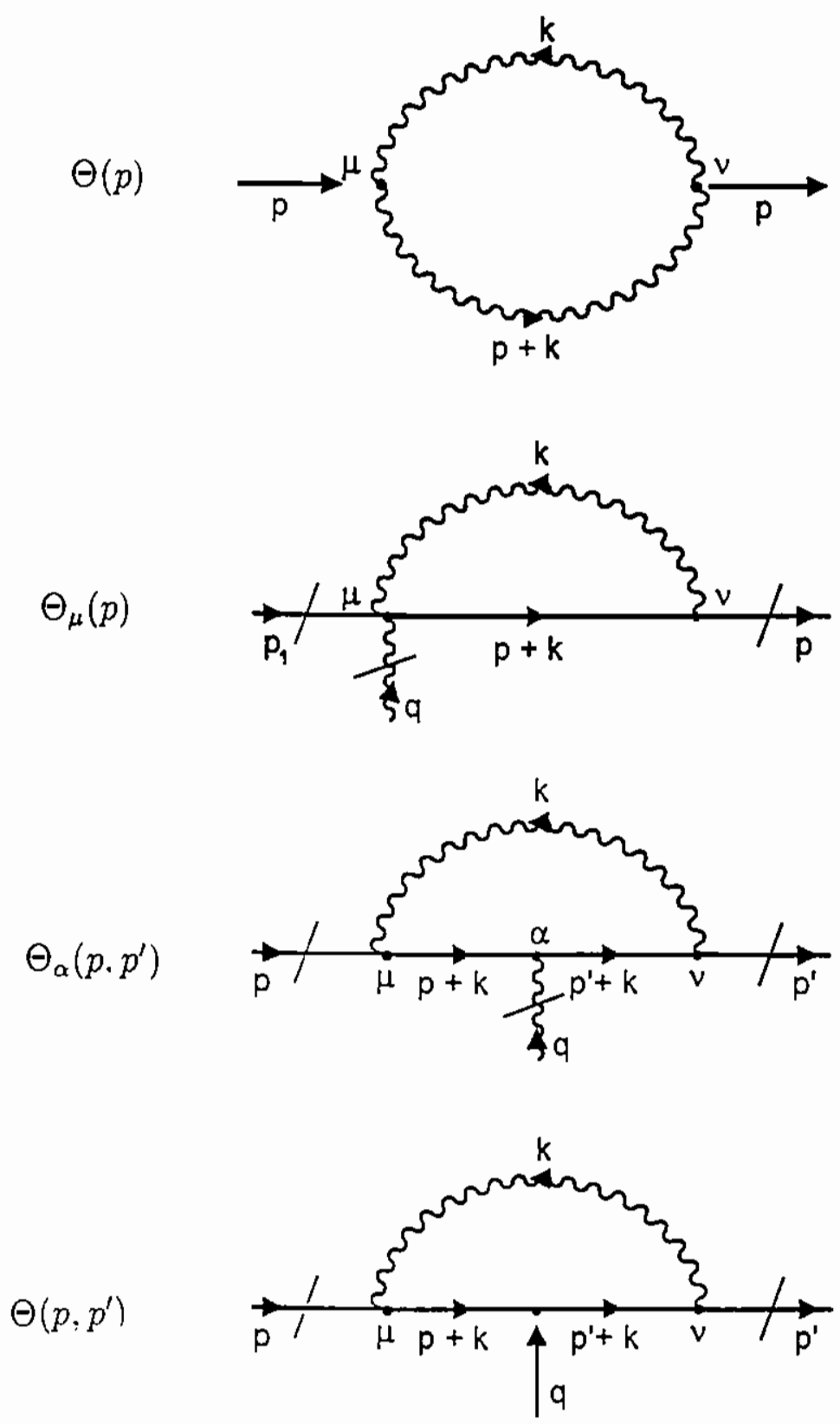

Figura 2.2: Diagramas $\Theta(p)$. $\Theta_{\mu}(p)$, com $p=p_{1}+q, \Theta_{\alpha}\left(p, p^{\prime}\right)$, com $p^{\prime}=p+q$, e $\Theta\left(p, p^{\prime}\right)$ $\operatorname{com} p^{\prime}=p+q$. 
A integral acima tem solução proporcional a $p_{\rho}$ (ver equação (B.2)), ou seja

$$
\Theta_{\mu}(p) \propto \varepsilon^{\mu \nu \rho} p_{\nu} p_{\rho}=0 .
$$

Para o diagrama $\Theta_{\alpha}\left(p, p^{\prime}\right)$ da figura (2.2), temos que

$$
\Theta_{\alpha}\left(p, p^{\prime}\right)=\int \mathcal{D} k \frac{i}{(p+k)^{2}} \frac{i}{\left(p^{\prime}+k\right)^{2}}(2 p+k)_{\mu}\left(2 p^{\prime}+k\right)_{\nu} \varepsilon^{\mu \nu \rho} \frac{k_{\rho}}{k^{2}}\left(2 k+p+p^{\prime}\right)_{\alpha}
$$

sendo que os termos proporcionais a $k_{\mu}$ e $k_{\nu}$ são nulos pela anti-simetria de $\varepsilon^{\mu \nu \rho} k_{\rho}$. Assim, a equação (2.28) reduz-se a

$$
\Theta_{\alpha}\left(p, p^{\prime}\right)=-4 \varepsilon^{\mu \nu \rho} p_{\mu} p_{\nu}^{\prime} \int \mathcal{D} k \frac{k_{\rho}\left(2 k+p+p^{\prime}\right)_{\alpha}}{k^{2}(p+k)^{2}\left(p^{\prime}+k\right)^{2}} .
$$

Fazendo a paramentrização de Feynman nesta integral usando (B.8) e escolhendo $A=$ $(p+k)^{2}, B=k^{2}$ e $C=\left(p^{\prime}+k\right)^{2}$, obtemos após alguma álgebra

$\Theta_{\alpha}\left(p, p^{\prime}\right)=\frac{8 \mu^{\epsilon}}{2^{d} \pi^{d}} \varepsilon^{\mu \nu \rho} p_{\mu} p_{\nu}^{\prime} \int_{0}^{1} d y y \int_{0}^{1} d x \int d^{d} k \frac{2 k_{\rho} k_{\alpha}+k_{\rho}\left(p+p^{\prime}\right)_{\alpha}}{\left[-k^{2}-2 k \cdot\left(p^{\prime} x+p(1-y)\right)-p^{2}(1-y)-p^{\prime 2} x\right]^{2}}$.

Integrando o segundo termo da integral acima vemos que será proporcional a $p_{\rho}$, que, pela anti-simetria de $\varepsilon^{\mu \nu \rho}$, será nulo. A integral do primeiro termo, usando (B.3), nos leva a

$$
\begin{aligned}
\Theta_{\alpha}\left(p, p^{\prime}\right)= & \frac{8 i \mu^{\epsilon}}{2^{d} \pi^{\frac{d}{2}}} \varepsilon^{\mu \nu \rho} p_{\mu} p_{\nu}^{\prime} \int_{0}^{1} d y y \int_{0}^{1} d x\left\{\Gamma\left(3-\frac{d}{2}\right) \frac{\left[p^{\prime} x+p(1-y)\right]_{\rho}\left[p^{\prime} x+p(1-y)\right]_{\alpha}}{\left[p^{\prime 2} x(x-1)+p^{2} y(y-1)+2 p^{\prime} \cdot p x(1-y)\right]^{3-}}\right. \\
& \left.-\frac{\Gamma\left(2-\frac{d}{2}\right)}{2} \frac{g_{\rho \alpha}}{\left[p^{\prime 2} x(x-1)+p^{2} y(y-1)+2 p^{\prime} \cdot p x(1-y)\right]^{2-\frac{d}{2}}}\right\} .
\end{aligned}
$$

o primeiro termo desta integral, novamente pela antissimetria do $\varepsilon^{\mu \nu \rho}$. é nulo. Assim

$$
\begin{aligned}
\Theta_{\alpha}\left(p, p^{\prime}\right)= & -\frac{4 i \mu^{\epsilon}}{2^{d} \pi^{\frac{d}{2}}} \Gamma\left(2-\frac{d}{2}\right) \varepsilon_{\alpha}^{\mu \nu} p_{\mu} p_{\nu}^{\prime}\left[p^{2}\right]^{\frac{d}{2}-2} \int_{0}^{1} d y y \int_{0}^{1} d x \\
& {\left[x(x-1)+\frac{p^{2}}{p^{\prime 2}} y(y-1)+\frac{2 p \cdot p^{\prime}}{p^{\prime 2}} x(1-y)\right]^{\frac{d}{2}-2} }
\end{aligned}
$$

na qual, fazendo-se as integrais em $x$ e $y$, temos que

$$
\Theta_{\alpha}\left(p, p^{\prime}\right)=\frac{i \mu^{\epsilon}}{2^{d} 3 \pi^{\frac{d}{2}}} \Gamma\left(2-\frac{d}{2}\right) \varepsilon_{\alpha}^{\mu \nu} p_{\mu} p_{\nu}^{\prime} \frac{\left(p+p^{\prime}\right)^{2}}{\left[p^{\prime 2}\right]^{\frac{3}{2}+\frac{e}{2}}}
$$


Para o gráfico $\Theta\left(p, p^{\prime}\right)$ da figura (2.2), temos que

$$
\Theta\left(p, p^{\prime}\right)=\int \mathcal{D} k \frac{i}{(p+k)^{2}} \frac{i}{\left(p^{\prime}+k\right)^{2}}(2 p+k)_{\mu}\left(2 p^{\prime}+k\right)_{\nu} \varepsilon^{\mu \nu \rho} \frac{k_{\rho}}{k^{2}},
$$

que pela anti-simetria do $\varepsilon^{\mu \nu \alpha}$ reduz-se simplesmente a

$$
\Theta\left(p, p^{\prime}\right)=-4 \varepsilon^{\mu \nu \rho} p_{\mu} p_{\nu}^{\prime} \int d^{d} k \frac{k_{\rho}}{k^{2}(p+k)^{2}\left(p^{\prime}+k\right)^{2}} .
$$

Implementando acima uma parametrização de Feynman da forma (B.8), chamando $A=$ $(p+k)^{2}, B=k^{2}$ e $C=\left(p^{\prime}+k\right)^{2}$ podemos escrever (2.35) como

$$
\Theta\left(p, p^{\prime}\right)=\frac{8 \mu^{\epsilon}}{2^{d} \pi^{d}} \varepsilon^{\mu \nu \rho} p_{\mu} p_{\nu}^{\prime} \int_{0}^{1} d y y \int_{0}^{1} d x \int d^{d} k \frac{k_{\rho}}{\left[-k^{2}-2 k \cdot\left(p^{\prime} x+p(1-y)\right)-p^{2}(1-y)-p^{\prime 2} x\right]^{2}},
$$

e usando (B.2) para resolver a integral em $k$ temos

$$
\Theta\left(p, p^{\prime}\right)=\frac{8 i \mu^{\epsilon}}{2^{d} \pi^{\frac{d}{2}}} \frac{\Gamma\left(3-\frac{d}{2}\right)}{\Gamma(3)} \varepsilon^{\mu \nu \rho} p_{\mu} p_{\nu}^{\prime} \int_{0}^{1} d y \int_{0}^{y} d x \frac{-\left[p^{\prime} x+p(1-y)\right]_{\rho}}{\left[-p^{2}(1-y)-p^{\prime 2} x+\left(p^{\prime} x+p(1-y)\right)^{2}\right]^{3-\frac{d}{2}}},
$$

da qual concluímos que

$$
\Theta\left(p, p^{\prime}\right) \propto \varepsilon^{\mu \nu \rho} p_{\mu} p_{\nu}^{\prime}\left(p^{\prime}+p\right)_{\rho}=0
$$

Vamos calcular agora um outro grupo de diagramas que podem ser vistos na figura (2.3).

O diagrama $\mathcal{A}_{1}$ pode ser escrito como

$$
\mathcal{A}_{1}=\int \mathcal{D} k \varepsilon^{\mu \nu \rho} \frac{k_{\rho}}{k^{2}} g_{\mu \nu}
$$

e pela anti-simetria de $\varepsilon^{\mu \nu \rho}$;

$$
\mathcal{A}_{1}=0 \text {. }
$$

Para o diagrama $\mathcal{A}_{2}$ temos

$$
\mathcal{A}_{2}(p)=\int \mathcal{D} k(2 p+k)_{\mu} \varepsilon^{\mu \nu \rho} \frac{k_{\rho}}{k^{2}}(2 p+k)_{\nu} \frac{i}{(p+k)^{2}},
$$

que pela antissimetria de $\varepsilon^{\mu \nu \rho}$ temos que

$$
\mathcal{A}_{2}(p)=0 .
$$



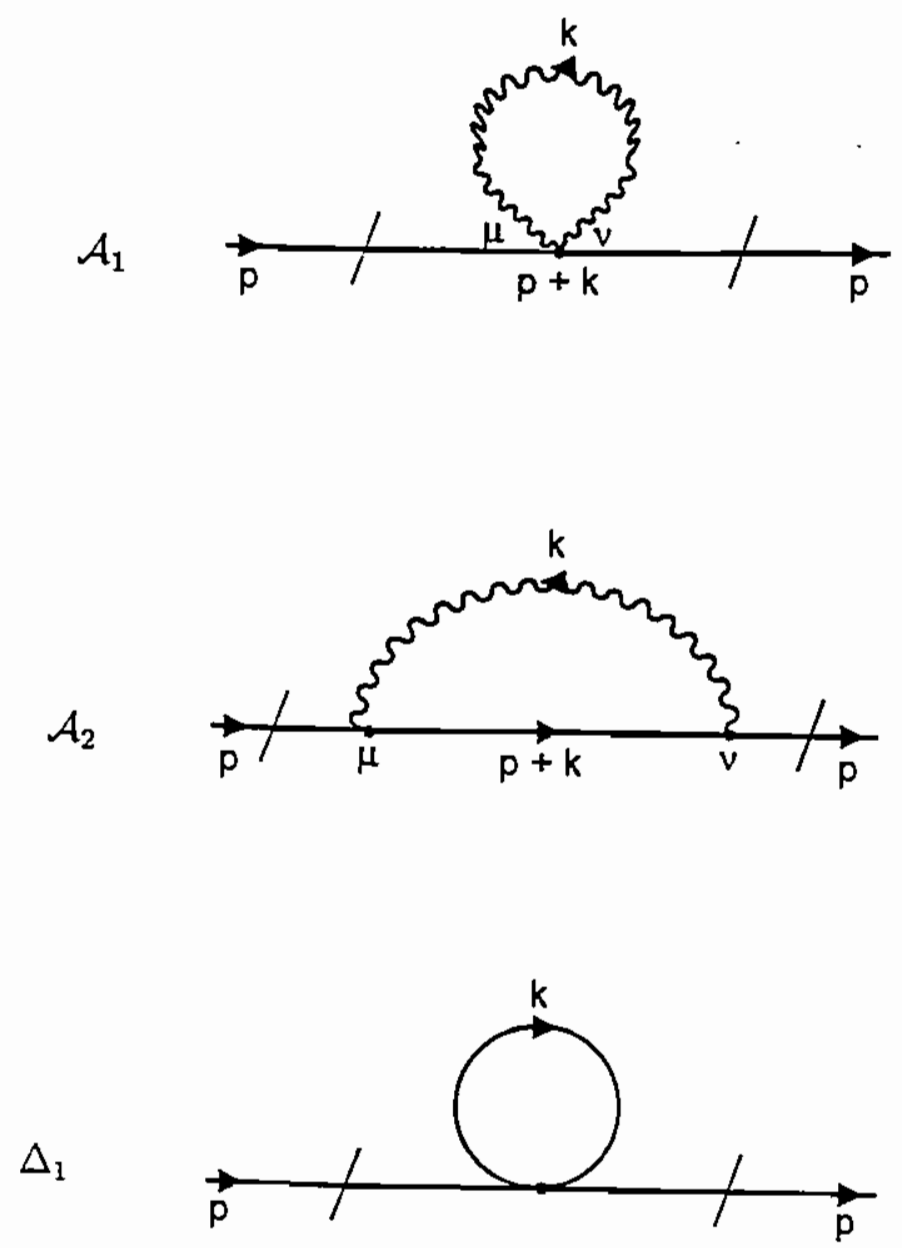

Figura 2.3: Diagramas $\mathcal{A}_{1}$. $\mathcal{A}_{2}$ e $\Delta_{1}$. 


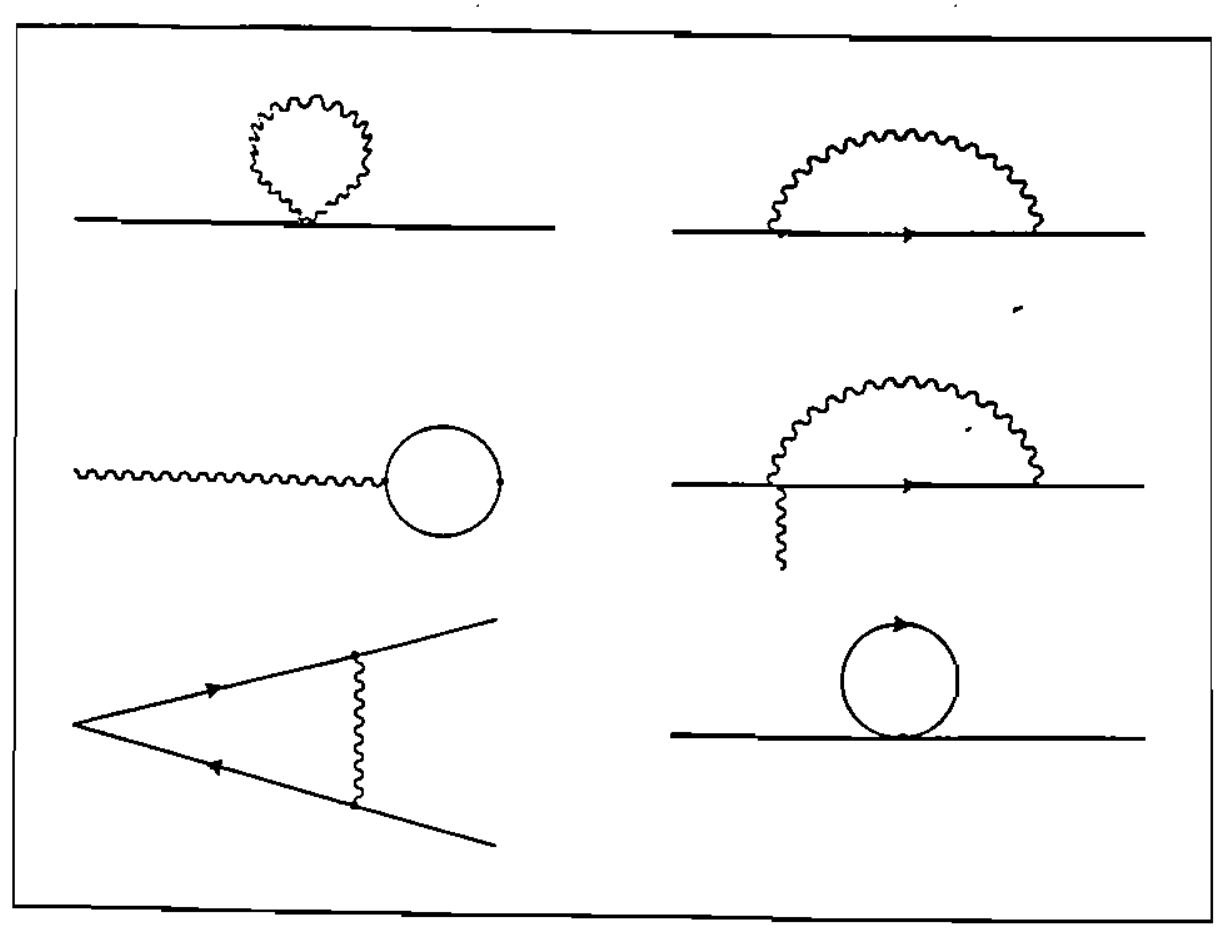

Figura 2.4: Diagramas nulos, respectivamente $\mathcal{A}_{1}, \mathcal{A}_{2}, \Pi_{\alpha}(p), \Theta_{\mu}(p)$ e $\Theta\left(p, p^{\prime}\right)$.

Completando o cálculo dos diagramas da figura (2.3), vamos calcular agora $\Delta_{1}$. Este diagrama é, algebricamente, dado por

$$
\Delta_{1}=\int \mathcal{D} k \frac{i}{k^{2}+i \eta}=-\frac{i \mu^{\epsilon}}{(2 \pi)^{d}} \int d^{d} k \frac{1}{-k^{2}-i \eta} .
$$

Sendo que na integral acima introduzimos um regulador $\eta$, de maneira a controlar uma divergência infravermelha da integral. Usando (B.1) em (2.43) temos

$$
\Delta_{1}=\frac{\mu^{\epsilon}}{(4 \pi)^{\frac{d}{2}}} \Gamma\left(1-\frac{d}{2}\right)(-i \eta)^{\frac{d}{2}-1}
$$

que: sendo $d / 2-1>0$ o regulador $\eta$ pode ser removido sem problemas. ou seja fazendo $\eta \rightarrow 0$ temos

$$
\Delta_{1}=0 \text {. }
$$

Resumindo. vemos que os diagramas $\Pi_{\alpha}(p)$, equação (2.12), $\Theta_{\mu}(p)$, equação (2.2 $\left.\bar{\tau}\right), \mathcal{A}_{1}$. equação (2.40). $\mathcal{A}_{2}$, equação (2.42). e $\Delta_{1}(p)$, equação (2.45), são nulos. Estes diagramas são mostrados na figura (2.4).

Com estes resultados nas mãos já podemos fazer uma primeira seleção dos diagramas. das funções de vértice de dois e seis pontos, que poderão colaborar para as funções do 


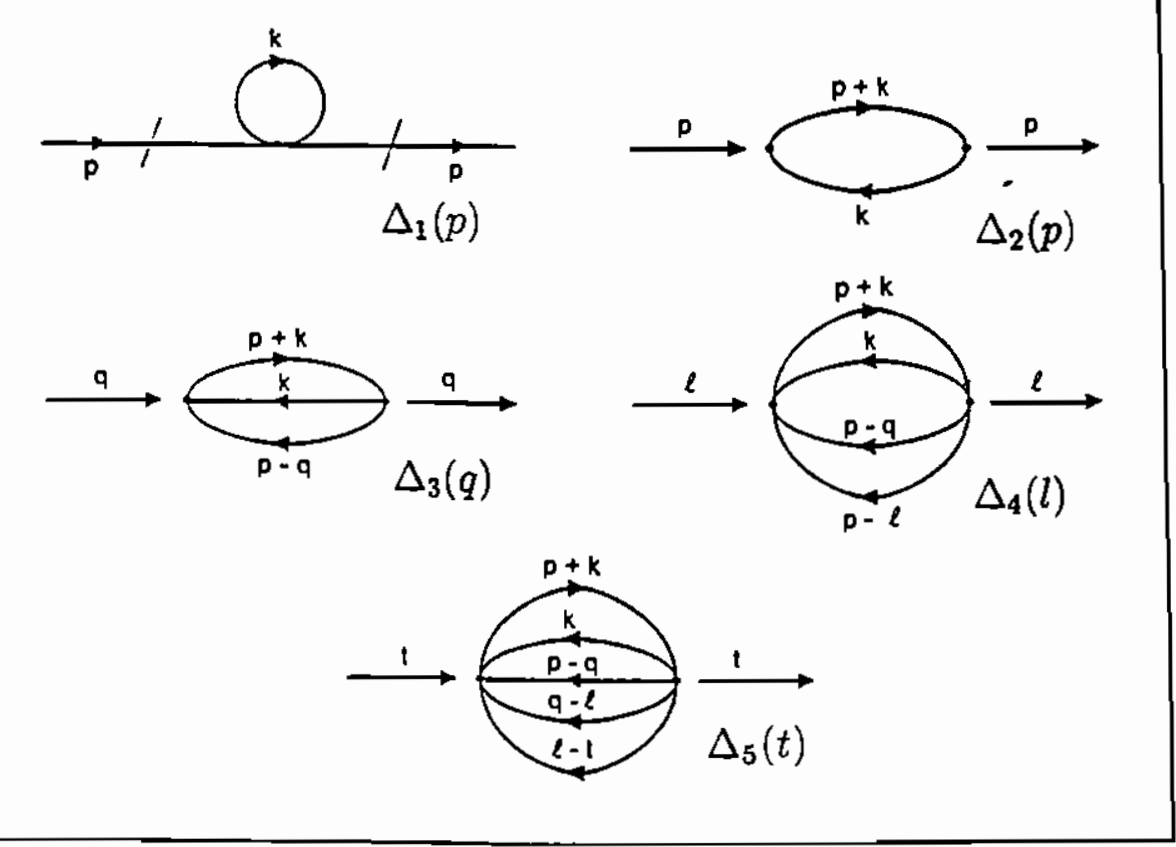

Figura 2.5: Diagramas $\Delta_{1}(p), \Delta_{2}(p) \Delta_{3}(q), \Delta_{4}(l)$ e $\Delta_{5}(t)$.

grupo de renormalização, ou, pelo menos, daqueles diagramas que não são identicamentes nulos.

Para a função de vértice de dois pontos já podemos descartar, além dos diagramas $\mathcal{A}_{1}$ e $\mathcal{A}_{2}$ (ver apêndice A), os diagramas $\mathcal{B}_{1}, \mathcal{C}_{4}, \mathcal{C}_{5}, \mathcal{C}_{7}, \mathcal{C}_{8}, \mathcal{C}_{9}, \mathcal{C}_{10}, \mathcal{D}_{2}, \mathcal{D}_{3}, \mathcal{D}_{4}, \mathcal{E}_{1}, \mathcal{E}_{2}, \mathcal{E}_{3}, \mathcal{E}_{4}$ e $\mathcal{E}_{5}$. Pode-se constatar que eles contém pelo menos um dos sub-diagramas nulos acima calculados. Desta forma nos resta calcular ainda os diagramas $\mathcal{C}_{1}, \mathcal{C}_{2}, \mathcal{C}_{3}, \mathcal{C}_{6}, \mathcal{C}_{9}$ e $\mathcal{D}_{1}$ : para a função de vértice de dois pontos.

Para a função de vértice de seis pontos já podemos descartar os diagramas $\mathcal{G}_{2}, \mathcal{H}_{1}$, $\mathcal{I}_{1}, \mathcal{I}_{3}, \mathcal{I}_{5}, \mathcal{I}_{7}, \mathcal{I}_{8}, \mathcal{I}_{9}, \mathcal{I}_{11}, \mathcal{I}_{12}, \mathcal{I}_{17}, \mathcal{I}_{18}, \mathcal{I}_{19}, \mathcal{I}_{20}, \mathcal{I}_{24}, \mathcal{I}_{25}, \mathcal{J}_{1}, \mathcal{J}_{2}, \mathcal{J}_{4}, \mathcal{J}_{5}, \mathcal{J}_{6}, \mathcal{J}_{7}, \mathcal{J}_{8}, \mathcal{J}_{9}$ $\mathcal{L}_{2}, \mathcal{L}_{3}, \mathcal{L}_{5}, \mathcal{L}_{6}, \mathcal{L}_{7}, \mathcal{L}_{8}, \mathcal{L}_{12}, \mathcal{L}_{13}, \mathcal{L}_{14}, \mathcal{L}_{15}, \mathcal{L}_{16}, \mathcal{L}_{17}, \mathcal{L}_{18}$, e $\mathcal{L}_{19}$. Aqui também, cada um dos diagramas acima comporta pelo menos um dos sub-diagramas nulos antes calculados. Desta forma nos resta calcular ainda os diagramas $\mathcal{G}_{1}, \mathcal{I}_{2}, \mathcal{I}_{4}, \mathcal{I}_{6}, \mathcal{I}_{10}, \mathcal{I}_{13} . \mathcal{I}_{14}, \mathcal{I}_{15}, \mathcal{I}_{16}$, $\mathcal{I}_{21}, \mathcal{I}_{22} . \mathcal{I}_{23}, \mathcal{J}_{3}, \mathcal{K}_{i}(i=1 \ldots .9) . \mathcal{L}_{1}, \mathcal{L}_{4}, \mathcal{L}_{9}, \mathcal{L}_{10}$ e $\mathcal{L}_{11}$, para a função de vértice de seis pontos.

Vamos agora calcular outro grupo de diagramas que serão úteis para o cálculo dos demais. Estes diagramas podem ser vistos na figura (2.5)

O diagrama $\Delta_{2}(p)$ pode ser escrito como

$$
\Delta_{2}(p)=\int \mathcal{D} k \frac{i}{k^{2}+i \eta} \frac{i}{(k+p)^{2}+i \eta}
$$


ou ainda, deixando o i $\eta$ subtendido, temos

$$
\Delta_{2}(p)=-\frac{\mu^{\epsilon}}{(2 \pi)^{d}} \int d^{d} k \frac{1}{\left[-k^{2}\right]} \frac{1}{\left[-(k+p)^{2}\right]},
$$

integral esta da forma $I_{(4)}$, equação (C.1), cuja solução pode ser vista em (C.4), que para $\alpha=\beta=1$, vê-se que

$$
\Delta_{2}(p)=-\frac{\mu^{\epsilon}}{2^{d} \pi^{\frac{d}{2}}\left[-p^{2}\right]^{\frac{d}{2}-2}} \frac{\Gamma\left(\frac{d}{2}-1\right)^{2} \Gamma\left(2-\frac{d}{2}\right)}{\Gamma^{2}(1) \Gamma(d-2)},
$$

a qual, escrevendo $d=3-\epsilon$, fica

$$
\Delta_{2}(p)=\frac{-i}{(4 \pi)^{\frac{3}{2}} \sqrt{-p^{2}}}\left(\frac{-4 \pi \mu^{2}}{p^{2}}\right)^{\frac{\epsilon}{2}} \frac{\Gamma\left(\frac{1}{2}-\frac{\epsilon}{2}\right)^{2} \Gamma\left(\frac{1}{2}+\frac{\epsilon}{2}\right)}{\Gamma(1-\epsilon)}
$$

ou ainda, calculando os valores das funções Gama segundo (B.10),

$$
\Delta_{2}(p)=\frac{-i}{8 \sqrt{-p^{2}}}\left(\frac{-4 \pi \mu^{2}}{p^{2}}\right)^{\frac{\epsilon}{2}} .
$$

O diagrama $\Delta_{3}(q)$ pode ser obtido tomando por base $\Delta_{2}(p)$ : pela inclusão de um novo propagador. Assim, o diagrama $\Delta_{3}(q)$ pode ser escrito como

$$
\Delta_{3}(q)=\int \mathcal{D} p \frac{i}{(p+q)^{2}}\left[\frac{i}{\left(-p^{2}\right)^{\frac{1}{2}+\frac{\epsilon}{2}}} \frac{\left(4 \pi \mu^{2}\right)^{\frac{\epsilon}{2}}}{(4 \pi)^{\frac{3}{2}}} \frac{\Gamma\left(\frac{1}{2}-\frac{\epsilon}{2}\right)^{2} \Gamma\left(\frac{1}{2}+\frac{\epsilon}{2}\right)}{\Gamma(1-\epsilon)}\right],
$$

sendo que o termo entre parenteses é o diagrama $\Delta_{2}$, conforme vemos em (2.49). A equação (2.51) pode ser escrita ainda da forma

$$
\Delta_{3}(q)=\frac{\left(4 \pi \mu^{2}\right)^{\frac{\epsilon}{2}}}{(4 \pi)^{\frac{3}{2}}} \frac{\Gamma\left(\frac{1}{2}-\frac{\epsilon}{2}\right)^{2} \Gamma\left(\frac{1}{2}+\frac{\epsilon}{2}\right)}{\Gamma(1-\epsilon)} \frac{\mu^{\epsilon}}{(2 \pi)^{d}} \int d^{d} p \frac{1}{\left[-(p+q)^{2}\right]} \frac{1}{\left[-p^{2}\right]^{\frac{1}{2}+\frac{\epsilon}{2}}} .
$$

A integral acima é da forma $I_{(4)}$, equação (C.1), cuja solução encontra-se em (C.4). Fazendo em (C.4) $\alpha=1 / 2+\epsilon / 2$ e $\beta=1$, podemos escrever (2.52) como

$$
\Delta_{3}(q)=\frac{-i}{(4 \pi)^{3}}\left(\frac{4 \pi \mu^{2}}{-q^{2}}\right)^{\epsilon} \frac{\Gamma\left(\frac{1}{2}-\frac{\epsilon}{2}\right)^{3}}{\Gamma\left(\frac{3}{2}-\frac{3 \epsilon}{2}\right)} \Gamma(\epsilon)
$$


Usando (B.10), B.11) e (B.12) temos que

$$
\begin{aligned}
\left(\frac{4 \pi \mu^{2}}{-q^{2}}\right)^{\epsilon} & =1+\epsilon \ln \left(\frac{4 \pi \mu^{2}}{-q^{2}}\right)+\mathcal{O}\left(\epsilon^{2}\right) \\
\Gamma\left(\frac{3}{2}-\frac{3 \epsilon}{2}\right)^{-1} & =\frac{2}{\pi^{\frac{1}{2}}}\left[1+\frac{3 \epsilon}{2}(2-\gamma-2 \ln 2)+\mathcal{O}\left(\epsilon^{2}\right)\right] \\
\Gamma\left(\frac{1}{2}-\frac{\epsilon}{2}\right)^{3} & =\pi^{\frac{3}{2}}\left[1+\frac{3 \epsilon}{2}(\gamma+2 \ln 2)+\mathcal{O}\left(\epsilon^{2}\right)\right] \\
\Gamma(\epsilon) & =\frac{1}{\epsilon}\left[1-\gamma \epsilon+\mathcal{O}\left(\epsilon^{2}\right)\right],
\end{aligned}
$$

substituindo estas equações em (2.53) temos que

$$
\Delta_{3}(q)=\frac{-i}{32 \pi^{2}}\left[\frac{1}{\epsilon}+\ln \left(\frac{4 \pi \mu^{2}}{-q^{2}-i \eta}\right)+3 \gamma+\mathcal{O}\left(\epsilon^{2}\right)\right],
$$

na qual reestabelecemos o regulador $\eta$, para controlar a divergência infra-vermelha.

Observe que para este diagrama, $\Delta_{3}$, calculamos a parte finita. Isto não será sempre necessário, já que apenas as partes divergentes dos diagramas irão compor as funções do grupo de renormalização. Porém veremos que existem diagramas de ordens superiores $\left(g^{3}\right)$ que terão pólos duplos, e estes diagramas serão compostos em parte, por sub-diagramas da forma de $\Delta_{3}$.

O diagrama $\Delta_{4}(l)$ pode ser escrito em termos de $\Delta_{3}$, bastando para tanto incluir um novo propagador em $\Delta_{3}$, como ilustra a figura (2.5). Obserrando isto podemos escrever que

$$
\Delta_{4}(l)=\int \mathcal{D} q \frac{i}{(q-l)^{2}}\left[\frac{-i}{\left(-q^{2}\right)^{\epsilon}} \frac{\left(4 \pi \mu^{2}\right)^{\epsilon}}{32 \pi^{2}} \Gamma(\epsilon)\right],
$$

sendo que o termo entre parenteses da equação acima é uma forma alternativa de escrever $\Delta_{3}(q)$ (fazendo a expansão $\epsilon$ das funções gama de (2.53), exceto $\Gamma(\epsilon)$ ). A equação (2.56) pode ainda ser escrita como

$$
\Delta_{4}(l)=-\frac{\Gamma(\epsilon)\left(4 \pi m^{2}\right)^{\epsilon}}{32 \pi^{2}} \int \mathcal{D} q \frac{1}{\left[-(q-l)^{2}\right]} \frac{1}{\left[-q^{2}\right]^{\epsilon}} .
$$

A integral em (2.57) possue a forma de $I_{(a)}$, equação (C.1). cuja solução encontra-se em (C.4). Fazendo em (C.4) $\alpha=\epsilon$ e $\beta=1$ temos que

$$
\Delta_{4}(l)=\frac{i \mu^{\epsilon}}{2^{d} \pi^{\frac{d}{2}}}\left(-\frac{\left(4 \pi \mu^{2}\right)^{\epsilon}}{32 \pi^{2}}\right)\left[-l^{2}\right]^{\frac{1}{2}-\frac{3 \epsilon}{2}} \frac{\Gamma\left(\frac{3}{2}-\frac{3 \epsilon}{2}\right) \Gamma\left(\frac{1}{2}-\frac{\epsilon}{2}\right) \Gamma\left(-\frac{1}{2}+\frac{3 \epsilon}{2}\right)}{\Gamma(2-2 \epsilon)},
$$


ou ainda, calculando os valores das funções Gamas, e introduzidno um regulador $\eta$ podemos escrever que

$$
\Delta_{4}(l)=\frac{i}{2^{8} \pi^{2}} \frac{\left(4 \pi \mu^{2}\right)^{\frac{3 \epsilon}{2}}}{\left(-l^{2}-i \eta\right)^{\frac{-1}{2}+\frac{3 \epsilon}{2}}}
$$

na qual, para $l \rightarrow 0$ e, em seguida, fazendo também $\eta \rightarrow 0$, temos $\Delta_{4} \rightarrow 0$.

Para obtermos $\Delta_{5}(t)$ devemos repetir o mesmo procedimento, ou seja, construí-lo a partir de $\Delta_{4}(l)$ pela inclusão de um novo propagador. Ou seja,

$$
\Delta_{5}(t)=\int \mathcal{D} l \frac{i}{(l-t)^{2}}\left[\frac{i}{\left(-l^{2}\right)^{-\frac{1}{2}+\frac{3 \epsilon}{2}}} \frac{\left(4 \pi \mu^{2}\right)^{\frac{3 \epsilon}{2}}}{2^{8} \pi^{2}}\right]
$$

na qual o termo entre parênteses é o diagrama $\Delta_{4}(l)$, equação (2.59). A equação (2.60) pode ainda ser escrita como

$$
\Delta_{5}(t)=\frac{\left(4 \pi \mu^{2}\right)^{\frac{3 \epsilon}{2}}}{2^{8} \pi^{2}} \frac{\mu^{\epsilon}}{(2 \pi)^{d}} \int d^{d} l \frac{1}{\left[-(l-t)^{2}\right]} \frac{1}{\left[-l^{2}\right]^{\frac{3 \epsilon}{2}-\frac{1}{2}}} .
$$

A integral acima já é nossa conhecida, (C.1), cuja solução está em (C.4), agora com $\alpha=3 \epsilon / 2-1 / 2$ e $\beta=1$. Feito isto $\Delta_{5}(t)$ assume a forma

$$
\Delta_{5}(t)=\frac{\left(4 \pi \mu^{2}\right)^{\frac{3 \epsilon}{2}} i \mu^{\epsilon}}{2^{d} \pi^{\frac{d}{2}}} \frac{[-t]^{1-2 \epsilon}}{2^{8} \pi^{2}} \frac{\Gamma\left(\frac{1}{2}-\frac{\epsilon}{2}\right) \Gamma(2-2 \epsilon) \Gamma(-1+2 \epsilon)}{\Gamma\left(\frac{3 \epsilon}{2}-\frac{1}{2}\right) \Gamma(1) \Gamma\left(\frac{5}{2}-\frac{5 \epsilon}{2}\right)}
$$

efetuando a expansão das funções Gamas segundo (B.10), resaltando o pólo contido em

$$
\Gamma(-1+2 \epsilon)=-\frac{1}{2 \epsilon}+\ldots
$$

o diagrama $\Delta_{5}(t)$ tem como resultado

$$
\Delta_{5}(t)=\frac{i}{3 \times 2^{11} \pi^{4}} \frac{\left(4 \pi \mu^{2}\right)^{2 \epsilon}}{\left(-t^{2}-i \eta\right)^{-1+2 \epsilon}} \frac{1}{\epsilon}+\text { parte finita }
$$

Os diagramas expostos na figura (2.j), e aqui calculados, serão úteis no cálculo das funções de vértice, como veremos a seguir. Estes resultados confirmam os obtidos por McKeon e Tsoupros[22]. 


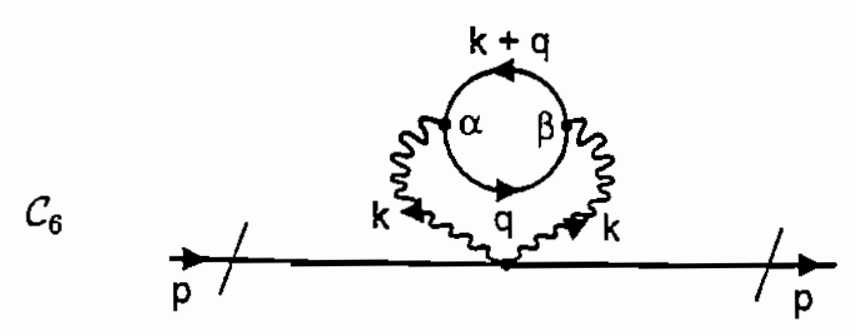

Figura 2.6: Diagramas $\mathcal{C}_{6}$, ordem $\alpha^{2}$.

\subsection{A Função de Vértice de Dois Pontos}

A função de vértice de dois pontos $\Gamma^{(2)}(p)$, ou função própria de dois pontos é definida por (2.7)

$$
\Gamma^{(2)}(p)=i p^{2}-i \Sigma(p)
$$

sendo que a auto-energia $-i \Sigma(p)$ é a soma dos gráficos irredutíveis de dois pontos, listadas até a ordem 2, no apêndice $A$.

Pela análise preliminar que fizemos na seção anterior, vimos que apenas os diagramas $\mathcal{C}_{1}, \mathcal{C}_{2}, \mathcal{C}_{3}, \mathcal{C}_{6}$, e $\mathcal{D}_{1}$ são, em princípio, diferentes de zero. Passaremos a analisar agora cada um deles.

O diagrama $\mathcal{C}_{6}$, que é visto na figura (2.6), pode ser escrito em termos do tensor de polarização $\Pi_{\alpha 3}(k)$, ou seja,

$$
\mathcal{C}_{6}=\int \mathcal{D} k \varepsilon^{\mu \alpha \rho} \frac{k_{\rho}}{k^{2}} \Pi_{\alpha \beta}(k) \varepsilon^{\gamma \beta}{ }_{\mu} \frac{k_{\gamma}}{k^{2}} .
$$

Substituindo o tensor de polarização $\Pi_{\alpha \beta}(k)$, equação (2.17), na expressão anterior temos que

$$
\mathcal{C}_{6}=\int \mathcal{D} k \varepsilon^{\mu \alpha \rho} \frac{k_{\rho}}{k^{2}}\left[-\frac{i}{16} \frac{\left(4 \pi \mu^{2}\right)^{\frac{\varepsilon}{2}}}{\left(-k^{2}\right)^{\frac{1}{2}+\frac{\epsilon}{2}}}\left(k_{\alpha} k_{3}-g_{\alpha 3} k^{2}\right)\right] \varepsilon^{3 \mu \gamma} \frac{k_{\gamma}}{k^{2}} .
$$

considerando na equação anterior que (baseados em (B.16)),

$$
\varepsilon^{\mu \alpha \rho} \Xi^{3 \mu \gamma} k_{\rho}\left(k_{\alpha} k_{\beta}-g_{\alpha \beta} k^{2}\right) k_{\gamma}=(d-1) k^{4},
$$


$\mathcal{C}_{1}$

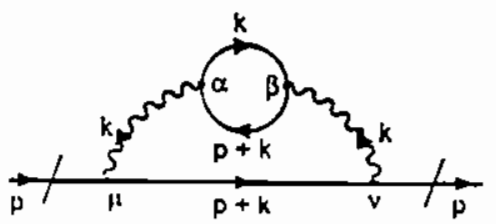

$\mathcal{C}_{2}$

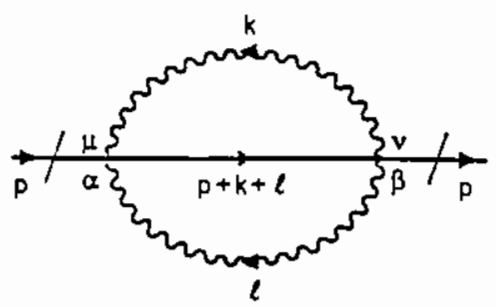

$\mathcal{C}_{3}$

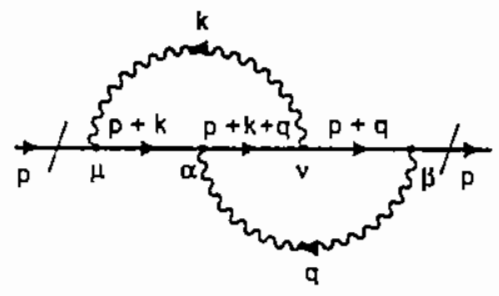

Figura 2.7: Diagramas $\mathcal{C}_{1}, \mathcal{C}_{2}$ e $\mathcal{C}_{3}$. Ordem $\alpha^{2}$

temos que

$$
\mathcal{C}_{6}=-\frac{i(d-1)\left(4 \pi \mu^{2}\right)^{\frac{\epsilon}{2}} \mu^{\epsilon}}{16(2 \pi)^{2}} I_{k}
$$

com

$$
I_{k}=\int d^{d} k \frac{1}{\left[-k^{2}+\eta\right]^{\frac{1}{2}+\frac{e}{2}}},
$$

a integral acima é da forma (B.1) e. quando o regulador vai para zero ela também anula-se. assim

$$
\mathcal{C}_{6}=0
$$

Vamos calcular agora os diagramas $\mathcal{C}_{1}, \mathcal{C}_{2}$ e $\mathcal{C}_{3}$, que podem ser vistos na figura (2.7)

$O$ diagrama $\mathcal{C}_{1}$ pode ser escrito em termos do tensor de polarização $\Pi_{\alpha 3}$. equação (2.17), ou. mais propriamente, em termos do diagrama $P_{\mu \nu}$, equação (2.19). no qual os propagadores de Chern-Simons encontram-se incluídos. Ou seja. podemos escrever $\mathcal{C}_{1}$ 
como

$$
\mathcal{C}_{\mathbf{l}}=\int \mathcal{D} k \frac{i}{(p+q)^{2}}(2 p+k)_{\mu}(2 p+k)_{\nu} \varepsilon^{\mu \alpha \rho} \frac{k_{\rho}}{k^{2}} \Pi_{\alpha \beta} \varepsilon^{\beta \nu \sigma} \frac{k_{\sigma}}{k^{2}}
$$

ou ainda, substituindo $\Pi_{\alpha \beta}$,

$$
\mathcal{C}_{1}=4 i \int \mathcal{D} k \frac{1}{(p+k)^{2}\left(k^{2}\right)^{2}} \varepsilon^{\mu \alpha \rho} \varepsilon^{\beta \nu \sigma} p_{\mu} p_{\nu} k_{\rho} k_{\sigma}\left[-\frac{1}{16 \sqrt{k^{2}}}\left(k_{\alpha} k_{\beta}-k^{2} g_{\alpha \beta}\right)\left(-\frac{4 \pi \mu^{2}}{k^{2}}\right)^{\frac{\epsilon}{2}}\right]
$$

que pode ser escrita da forma

$$
\mathcal{C}_{1}=\frac{\left(4 \pi \mu^{2}\right) \mu^{\epsilon}\left(p^{2} g^{\mu \nu}-p^{\mu} p^{\nu}\right)}{2^{d+2} \pi^{d}} I_{k}
$$

sendo

$$
I_{k}=\int d^{d} k \frac{k_{\mu} k_{\nu}}{\left[-k^{2}\right]^{\frac{3}{2}+\frac{\epsilon}{2}}\left[-(p+k)^{2}\right]}
$$

Esta integral em $k$ é do tipo (C.3), cuja solução é mostrada em (C.6), que para $\alpha=$ $3 / 2+\epsilon / 2$ e $\beta=1$,

$$
I_{k}=i \pi^{\frac{d}{2}} \frac{\Gamma\left(\frac{d}{2}-2\right) \Gamma\left(\frac{d}{2}\right) \Gamma\left(3-\frac{d}{2}\right)}{2 \Gamma\left(3-\frac{d}{2}\right) \Gamma\left(\frac{d}{2}-2\right)}\left(-p^{2}\right)^{d-3}\left[\frac{2(3-d)(d-2)}{\left(\frac{d}{2}-1\right)} \frac{p_{\mu} p_{\nu}}{p^{2}}-g_{\mu \nu}\right]
$$

$O$ primeiro termo do fator entre parênteses é nulo para $\epsilon \rightarrow 0(d=3)$. Usando ainda (B.10) para o cálculo das funções gamas, substituindo (2.76) em (2.74) e fazendo as contrações dos índices de Lorentz temos

$$
\mathcal{C}_{1}(p)=-\frac{i p^{2}}{24 \pi^{2}}\left[-\frac{4 \pi \mu^{2}}{p^{2}}\right]^{\epsilon} \Gamma(3-d)
$$

Se escrevermos $\Gamma(3-d)$ como $\Gamma(\epsilon)$ e fazermos a expansão desta em torno de $\epsilon=0$ conforme (B.10), podemos escrever finalmente que

$$
\mathcal{C}_{1}(p)=-\frac{i p^{2}}{24 \pi^{2}} \frac{p^{2}}{\epsilon}+\text { parte finita }
$$

Já sabemos que a parte finita não é de interesse para nós, pois. como vimos ao analizarmos as funções do grupo de renormalização, apenas os termos de pólo compõem tais funções. 
Vamos agora calcular o diagrama $\mathcal{C}_{2}$ da figura (2.7). O diagrama $\mathcal{C}_{2}$ pode ser escrito em termos do diagrama $\Theta(p)$, figura (2.2), que representa um "loop" composto de propagadores de Chern-Simons, da seguinte maneira

$$
\mathcal{C}_{2}=\int \mathcal{D} q \frac{i}{(p+q)^{2}} \Theta(q)
$$

Usando o resultado para $\Theta(p)$, equação (2.24), temos que

$$
\mathcal{C}_{2}=\int \mathcal{D} q \frac{i}{(p+q)^{2}}\left[-\frac{i}{8} \frac{\left(4 \pi \mu^{2}\right)^{\frac{\epsilon}{2}}}{\left(-q^{2}\right)^{-\frac{1}{2}+\frac{\epsilon}{2}}}\right]
$$

a qual podemos escrever como

$$
\mathcal{C}_{2}=-\frac{i}{8} \frac{\left(4 \pi \mu^{2}\right) \mu^{\frac{3 k}{2}}}{2^{d} \pi^{d}} I_{q}
$$

sendo

$$
I_{q}=\int d^{d} q \frac{1}{\left[-q^{2}\right]^{-\frac{1}{2}+\frac{\varepsilon}{2}}\left[-(p+q)^{2}\right]} .
$$

Esta integral em $q$ é da forma (C.1), cuja solução encontra-se em (C.4). Para $\alpha=$ $-1 / 2+\epsilon / 2$ e $\beta=1$ e aproximando as funções gamas temos

$$
I_{q}=i \pi^{\frac{d}{2}}\left(-p^{2}\right)^{1-\frac{e}{2}} \frac{\Gamma\left(\frac{2 d-2}{2}\right) \Gamma\left(\frac{d-2}{2}\right) \Gamma\left(\frac{4-2 d}{2}\right)}{\Gamma\left(\frac{2-d-}{2}\right) \Gamma\left(\frac{3 d-4}{2}\right)}=\frac{-2 i \pi^{\frac{d}{2}-\frac{1}{2}}}{3}\left(-p^{2}\right)^{1-\frac{\epsilon}{2}} \Gamma(-1+\epsilon) .
$$

Substituindo $I_{q}$ na equação (2.81) temos

$$
\mathcal{C}_{2}=-\frac{i p^{2}}{96 \pi^{2}}\left[-\frac{\left(4 \pi \mu^{2}\right)^{2}}{p^{2}}\right]^{\frac{\ell}{2}} \Gamma(-1+\epsilon)
$$

e fazendo a expansão em $\epsilon$ como em (B.10), escrevemos finalmente $\mathcal{C}_{2}$ como

$$
\mathcal{C}_{2}=\frac{i}{96 \pi^{2}} \frac{p^{2}}{\epsilon}+\text { parte finita } .
$$

Vamos calcular agora o diagrama $\mathcal{C}_{3}$, mostrado na figura (2.7). Este diagrama pode ser escrito em termos de $\Theta_{\alpha}\left(p, p^{\prime}\right)$ da figura (2.5). Isto é feito da seguinte maneira

$$
\mathcal{C}_{3}(p)=\int \mathcal{D} q \Theta_{\alpha}(p, p+q) \varepsilon^{\alpha \beta \gamma} \frac{q_{\gamma}}{q^{2}}(2 p+q)_{3} \frac{i}{(p+q)^{2}} .
$$


Substituindo $\Theta_{\alpha}$, equação (2.32) em (2.86) temos

$$
\begin{aligned}
\mathcal{C}_{3}(p)= & \frac{8 \mu^{\epsilon}}{2^{d} \pi^{\frac{d}{2}}} \Gamma\left(2-\frac{d}{2}\right) \int \mathcal{D} q \int_{0}^{1} d y y \int_{0}^{1} d x \varepsilon^{\mu \nu}{ }_{\alpha} \varepsilon^{\alpha \beta \gamma} \frac{p_{\mu}(p+q)_{\nu} q_{\gamma} p_{\beta}}{q^{2}(p+q)^{2}} \\
& {\left[(p+q)^{2} x(x-1)+p^{2} y(y-1)+2(p+q) \cdot p x(1-y)\right]^{\frac{d}{2}-2} . }
\end{aligned}
$$

Usando que $\varepsilon_{\alpha}{ }^{\mu \nu} \varepsilon^{\alpha \beta \gamma}=g^{\mu \beta} g^{\nu \gamma}-g^{\mu \gamma} g^{\nu \beta}$ obtemos

$$
\begin{aligned}
\mathcal{C}_{3}(p)= & \frac{8 \mu^{\epsilon}}{2^{d} \pi^{\frac{d}{2}}} \Gamma\left(2-\frac{d}{2}\right) \int \mathcal{D} q \int_{0}^{1} d y y \int_{0}^{1} d x\left(p^{2} g^{\alpha \beta}-p^{\alpha} p^{\beta}\right) q_{\alpha} q_{\beta} \\
& \frac{1}{q^{2}(p+q)^{2}\left[(p+q)^{2} x(x-1)+p^{2} y(y-1)+2(p+q) \cdot p x(1-y)\right]^{2-\frac{d}{2}}}
\end{aligned}
$$

Aqui devemos fazer duas parametrizações. A primeira (B.7)

$$
\frac{1}{A B}=\int_{0}^{1} d z \frac{1}{[A z+B(1-z)]^{2}}
$$

com $A=q^{2}$ e $B=(p+q)^{2}$, e a segunda

$$
\frac{1}{A^{\alpha} B^{\beta}}=\frac{\Gamma(\alpha+\beta)}{\Gamma(\alpha) \Gamma(\beta)} \int_{0}^{1} d w \frac{w^{\alpha-1}(1-w)^{\beta-1}}{[A w+B(1-w)]^{\alpha+\beta}}
$$

sendo

$$
\begin{aligned}
& A=(p+q)^{2} x(x-1)+p^{2} y(y-1)+2(p+q) \cdot p x(1-y) \\
& B=q^{2} z+(p+q)^{2}(1-z)
\end{aligned}
$$

e assim, já escrevendo $\mathcal{D} q=\frac{\mu^{c}}{2^{d} \pi^{d}} d^{d} q$, temos que

$$
\begin{aligned}
\mathcal{C}_{3}(p)= & \frac{8 \mu^{2 \epsilon}}{2^{2 d} \pi^{\frac{3 d}{2}}} \Gamma\left(4-\frac{d}{2}\right) \int_{0}^{1} d y y \int_{0}^{1} d x \int_{0}^{1} d z \int_{0}^{1} d w w^{1-\frac{d}{2}}(1-w) \\
& \int d^{d} q\left(p^{2} g^{\alpha \beta}-p^{\alpha} p^{\beta}\right) q_{\alpha} q_{\beta}\left[w x(x-1)(p+q)^{2}+w y(y-1) p^{2}\right. \\
& \left.+w x(1-y) 2(p+q) \cdot p+(1-w) q^{2} z+(1-w)(1-z)(p+q)^{2}\right]^{\frac{d}{2}-4} .
\end{aligned}
$$

Esta equação pode ainda ser reescrita como

$$
\mathcal{C}_{3}(p)=\frac{8 \mu^{2 \epsilon}}{2^{2 d} \pi^{\frac{3 d}{2}}} \Gamma\left(4-\frac{d}{2}\right)\left(p^{2} g^{\alpha \beta}-p^{\alpha} p^{\beta}\right) \int_{0}^{1} d y y \int_{0}^{1} d x \int_{0}^{1} d z \int_{0}^{1} d w w^{1-\frac{d}{2}}(1-w) \mathcal{I}_{\alpha, 3}
$$


sendo

$$
\mathcal{I}_{\alpha \beta}=\frac{1}{(-a)^{4-\frac{d}{2}}} \int d^{d} q \frac{q_{\alpha} q_{\beta}}{\left[-q^{2}-2 q \cdot p \frac{a}{b}-p^{2} \frac{c}{a}\right]^{4-\frac{d}{2}}}
$$

com

$$
\begin{aligned}
a & =1-w+w x(x-1) \\
b & =w x^{2}-w x y+(1-w)(1-z) \\
c & =w x(x-1)+w y(y-1)+(1-z)(1-w)+2 w x(1-y) .
\end{aligned}
$$

A integral $\mathcal{I}_{\alpha \beta}$ tem a forma de (B.3), e tem como solução

$$
\mathcal{I}_{\alpha \beta}=i \pi^{\frac{d}{2}}(-a)^{\frac{d}{2}-4}\left\{\frac{\Gamma(4-d)}{\Gamma\left(4-\frac{d}{2}\right)} \frac{\left(\frac{b}{a}\right)^{2} p_{\alpha} p_{\beta}}{\left[\left(\frac{b}{a}\right)^{2} p^{2}-\left(\frac{b}{a}\right) p^{2}\right]^{4-d}}-\frac{\Gamma(3-d)}{2 \Gamma\left(4-\frac{d}{2}\right)} \frac{g_{\alpha \beta}}{\left[\left(\frac{b}{a}\right)^{2} p^{2}-\left(\frac{b}{a}\right) p^{2}\right]^{4-d}}\right\}
$$

Tendo o resultado para $\mathcal{I}_{\alpha \beta}$ podemos fazer

$$
\left(p^{2} g^{\alpha \beta}-p^{\alpha} p^{\beta}\right) \mathcal{I}_{\alpha \beta}=-i \pi^{\frac{d}{2}}(-a)^{\frac{d}{2}-4} \frac{\Gamma(3-d)}{2 \Gamma\left(4-\frac{d}{2}\right)}(d-1) \frac{p^{2}}{\left[\left(\frac{b}{a}\right)^{2} p^{2}-\left(\frac{b}{a}\right) p^{2}\right]^{3-d}\left(p^{2}\right)^{3-d}}
$$

e para $\epsilon=3-d$ em torno de zero, $\mathcal{C}_{3}$ torna-se

$$
\begin{aligned}
\mathcal{C}_{3}(p)= & \frac{-4 i \mu^{2 \epsilon}}{2^{2 d} \pi^{d}}(d-1) \Gamma(\epsilon) \frac{p^{2}}{\left(p^{2}\right)^{\epsilon}} \int_{0}^{1} d w w^{1-\frac{d}{2}} \int_{0}^{1} d y y \int_{0}^{1} d z \int_{0}^{1} d x(-a)^{\frac{d}{2}-4} \\
& {\left[1+\epsilon \ln \left(\frac{b^{2}-a c}{a^{2}}\right)+\ldots\right] }
\end{aligned}
$$

ou ainda

$$
\mathcal{C}_{3}(p)=\frac{-i 8}{(4 \pi)^{3}} p^{2} I\left(\frac{1}{\epsilon}+\ldots\right)
$$

sendo $I$ o conjunto de todas as integrais paramétricas que. substituindo o valor de $a$ dado por (2.95) será

$$
I=\int_{0}^{1} d w w^{1-\frac{d}{2}} \int_{0}^{1} d y y \int_{0}^{1} d z \int_{0}^{1} d x[w-1+w x(1-x)]^{\frac{d}{2}-4}=\frac{2 \pi}{3}
$$


que, levando até (2.101) temos finalmente que o diagrama $\mathcal{C}_{3}$ será

$$
\mathcal{C}_{3}(p)=-\frac{i p^{2}}{12 \pi^{2}} \frac{1}{\epsilon}+\text { parte finita } .
$$

O último diagrama que colabora para a função de dois pontos que resta ser analisado é o gráfico $\mathcal{D}_{1}$, que é equivalente ao diagrama $\Delta_{5}(t)$ da figura (2.5). Assim, por (2.64), podemos escrever que

$$
\mathcal{D}_{1}=\Delta_{5}(p)=-\frac{i p^{2}}{3.2^{11} \pi^{4}} \frac{1}{\epsilon}+\text { parte finita } .
$$

Portanto, tendo em mãos os resultados para as amplitudes $\mathcal{C}_{1}$ (equação $(2.78)$ ), $\mathcal{C}_{2}$ (equação $(2.85)$ ), $\mathcal{C}_{3}$ (equação $(2.103)$ ) e $\mathcal{D}_{1}$ (equação (2.104)),

$$
\begin{aligned}
\mathcal{C}_{1}(p) & =-\frac{i}{24 \pi^{2}} \frac{p^{2}}{\epsilon}+\text { parte finita } \\
\mathcal{C}_{2} & =\frac{i}{96 \pi^{2}} \frac{p^{2}}{\epsilon}+\text { parte finita } \\
\mathcal{C}_{3}(p) & =-\frac{i}{12 \pi^{2}} \frac{p^{2}}{\epsilon}+\text { parte finita } \\
\mathcal{D}_{1} & =-\frac{i}{3 \times 2^{11} \pi^{4}} \frac{p^{2}}{\epsilon}+\text { parte finita } .
\end{aligned}
$$

podemos escrever a seguinte tabela

\begin{tabular}{||c|c|c|c||}
\hline Diagramas & Parte Divergente $(1 / \epsilon)$ & Acoplamento & Fator Combinatorial \\
\hline \hline $\mathcal{C}_{1}$ & $\frac{-i p^{2}}{24 \pi^{2}}$ & $\frac{(-i e)^{4}}{4 !}$ & $4 !$ \\
\hline $\mathcal{C}_{2}$ & $\frac{i p^{2}}{96 \pi^{2}}$ & $\frac{\left(i e^{2}\right)^{2}}{2 !}$ & $2^{2}$ \\
\hline $\mathcal{C}_{3}$ & $\frac{-i p^{2}}{12 \pi^{2}}$ & $\frac{(-i e)^{4}}{4 !}$ & $4 !$ \\
\hline $\mathcal{D}_{1}$ & $\frac{-i p^{2}}{3 \times 2^{11} \pi^{4}}$ & $\frac{(-i g)^{2}}{2 !(3 !)^{4}}$ & $(3 !)^{3}$ \\
\hline
\end{tabular}

Tabela 2.1 - Partes divergentes, acoplamentos e fatores combinatoriais dos 


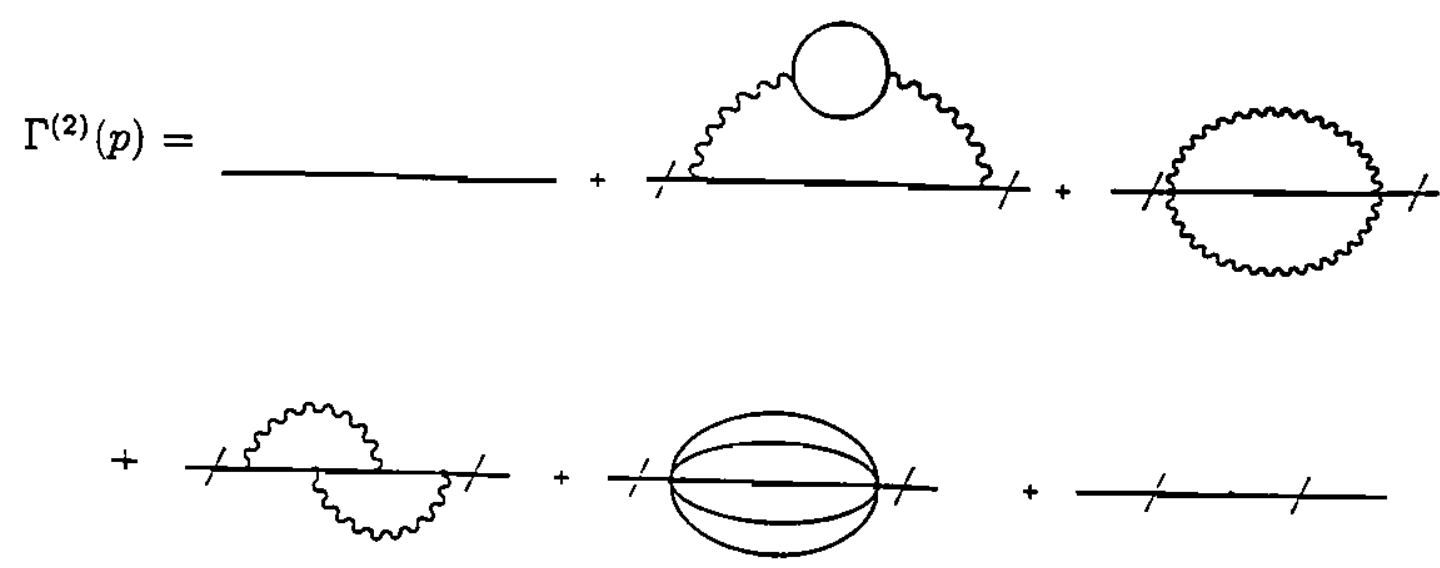

Figura 2.8: Função de vértice de dois pontos. O último termo da série é o contratermo para a função de dois pontos, e que é, em princípio a soma $A=A_{1}+A_{2}$, sendo $A_{1}$ os contratermos de ordem 1 e $A_{2}$ os contratermos de ordem 2.

diagramas que contribuem para a parte divergente da função de dois pontos.

A função de vértice de dois pontos, conforme definida em (2.7) será então dada diagramaticamente pela figura (2.8)

Algebricamente a função de dois pontos será, segundo a tabela (2.1),

$$
\begin{aligned}
\Gamma^{(2)}(p) & =i p^{2}-e^{4} \frac{i p^{2}}{24 \pi^{2}} \frac{1}{\epsilon}-e^{4} \frac{2 i p^{2}}{96 \pi^{2}} \frac{1}{\epsilon}-e^{4} \frac{i p^{2}}{12 \pi^{2}} \frac{1}{\epsilon}+g^{2} \frac{1}{3.2^{2}} \frac{i p^{2}}{3.2^{11} \pi^{2}} \frac{1}{\epsilon} \\
& +i\left(A_{1}+A_{2}\right) p^{2}+i p^{2} \text { (contribuição finita) }
\end{aligned}
$$

A "contribuição finita" na equação acima representa a soma das partes finitas de cada diagrama. Como não temos nenhuma divergência de ordem $1\left(e^{2}\right.$ ou $\left.g\right)$ temos que

$$
A_{1}=0 \text {. }
$$

Somando as contribuições divergentes em (2.109) temos que

$$
\Gamma^{(2)}(p)=i p^{2}\left[1+. A_{2}-\frac{\tau}{48 \pi^{2}} e^{4} \frac{1}{\epsilon}+\frac{g^{2}}{3^{2} 2^{13} \pi^{4}} \frac{1}{\epsilon}+\text { (contribuição finita) }\right] .
$$

da qual concluimos que $t_{2}$ deve ser

$$
A_{2}=\left[\frac{\tau}{48 \pi^{2}} \alpha^{2}-\frac{g^{2}}{3^{2} 2^{13} \pi^{4}}\right] \frac{1}{\epsilon},
$$

sendo este o contratermo necessário para tornar finita a função de vértice. Observe que fizemos com que $\alpha=e^{2}$. ou seja. estamos contando na expansão perturbativa em ondens do coeficiente do terno de Chern-Simons $\alpha$ e do acoplamento $g$. 


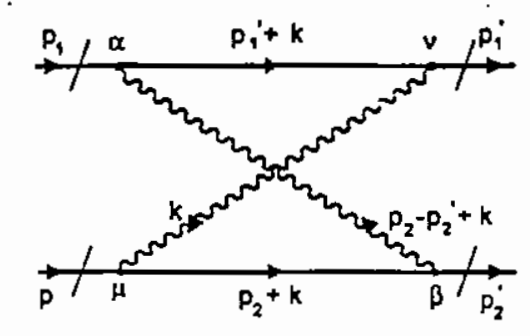

$\Omega$

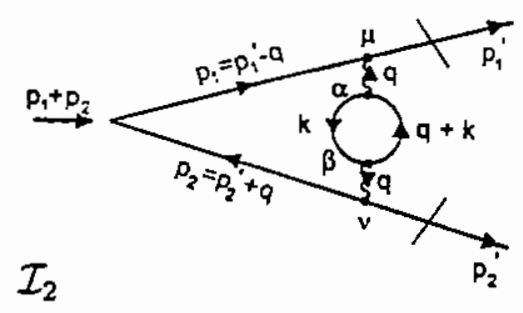

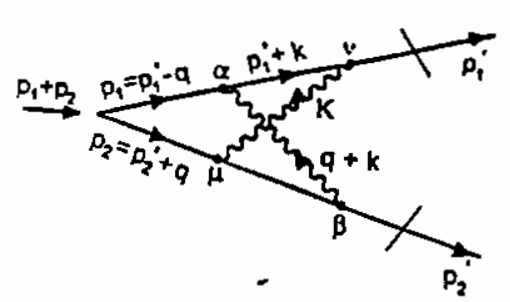

$\mathcal{I}_{4}$

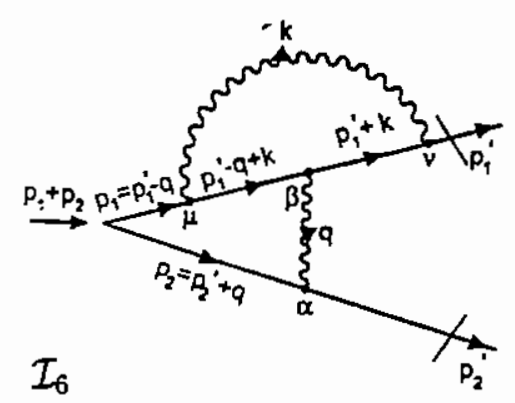

Figura 2.9: Diagramas $\Omega, \mathcal{I}_{2}, \mathcal{I}_{4}$ e $\mathcal{I}_{6}$. O diagrama $\Omega$ é um sub-diagrama de $\mathcal{I}_{4}$.

\subsection{A Função de Vértice de Seis Pontos}

A função de vértice de seis pontos $\Gamma^{(6)}$, ou função própria de seis ponto é definida por (2.8)

$$
\Gamma^{(6)}=\Delta^{(2)}\left(p_{1}\right)^{-1} \ldots \Delta^{(2)}\left(p_{6}\right)^{-1} G_{c}^{(6)}\left(p_{1}, \ldots, p_{6}\right)
$$

sendo $G_{c}^{(6)}$ a função de Green conexa de seis pontos, e $\Delta^{(2)}\left(p_{1}\right)^{-1}$ significa que os campos externos dever ser excluídos. A função de Green conexa de seis pontos é a soma de todas as amplitudes de Feynman de seis pontos. Aqui levaremos a teoria de pertubação até a primeira ordem não trivial, ou seja, até que as amplitudes de Feynman apresentem singularidades. Isto acontece, como veremos. na ordem 3 das constantes de acoplamento.

$\mathrm{Na}$ ordem $g^{3}$ algumas amplitudes exibem pólos duplos. os quais, como vimos ao analisar as funçōes do grupo de renormalização, não influencian tais funçōes. Porém. por completeza: explicitaremos aqui o cálculo destes pólos.

Os diagramas que colaboram para a função de seis pontos estão listadas no apêndice A. No final da seção 2.2 fomos capazes de descartar alguns destes diagramas por serem identicamente nulos. Assim feito nos restam, no entanto, os seguintes diagramas: $\mathcal{G}_{1}, \mathcal{I}_{2}$, $\mathcal{I}_{4}, \mathcal{I}_{6}, \mathcal{I}_{10}, \mathcal{I}_{13} . \mathcal{I}_{1: 1}, \mathcal{I}_{15}, \mathcal{I}_{16}, \mathcal{I}_{21}, \mathcal{I}_{22}, \mathcal{I}_{23}, \mathcal{J}_{3}, \mathcal{K}_{i}(i=1, \ldots, 9), \mathcal{L}_{1}, \mathcal{L}_{4}, \mathcal{L}_{9}, \mathcal{L}_{10}$ e $\mathcal{L}_{11}$, que passaremos a analisar agora.

Vamos dar início a esta análise como grupo de diagramas listados na figura (2.9). O primeiro chamaremos de $\Omega\left(p_{1}^{\prime}, p_{2}\right)$ (um sub-diagrama de $\mathcal{I}_{4}$ )e os demais são dos diagramas $\mathcal{I}_{2}, \mathcal{I}_{4}$ e $\mathcal{I}_{6}$

O diagrama $\Omega\left(p_{1}, p_{2}, p_{1}^{\prime}, p_{2}^{\prime}\right)$ pode ser escrito como 


$$
\begin{aligned}
\Omega\left(p_{1}, p_{2}, p_{1}^{\prime}, p_{2}^{\prime}\right)= & \int \mathcal{D} k \frac{i}{\left(p_{2}+k\right)^{2}} \frac{i}{\left(p_{1}^{\prime}+k\right)^{2}} \varepsilon^{\alpha \beta \gamma} \frac{\left(p_{2}-p_{2}^{\prime}+k\right)_{\gamma}}{\left(p_{2}-p_{2}^{\prime}+k\right)^{2}} \varepsilon^{\mu \nu \rho} \frac{k_{\rho}}{k^{2}} \\
& \left(p_{1}+p_{1}^{\prime}+k\right)_{\alpha}\left(2 p_{1}^{\prime}+k\right)_{\nu}\left(2 p_{2}+k\right)_{\mu}\left(p_{2}+p_{2}^{\prime}+k\right)_{\beta}
\end{aligned}
$$

que após alguma álgebra, multiplicando os termos acima e usando as propriedades de anti-simetria dos tensores de Levi-Civita, podemos escrever

$$
\Omega=-16 \varepsilon^{\alpha \beta \gamma} \varepsilon^{\mu \nu \rho} p_{1 \alpha} p_{1 \nu}^{\prime} p_{2 \mu} p_{2 \beta}^{\prime} \int \mathcal{D} k \frac{k_{\rho}\left(p_{2}-p_{2}^{\prime}+k\right)_{\gamma}}{k^{2}\left(p_{2}+k\right)^{2}\left(p_{1}^{\prime}+k\right)^{2}\left(p_{2}-p_{2}^{\prime}+k\right)^{2}} .
$$

o grau de divergência superficial para a equação anterior é $d_{k}(\Omega)=-3$, sendo portanto finito. Considerando que $p_{1}$ e $p_{2}$ também possam tenter para infinito temos que $d_{k, p_{i}}(\Omega)=$ -1 , sendo, portanto, também finito.

O diagrama $\mathcal{I}_{4}$ pode ser escrito em termos de $\Omega_{1}\left(p_{1}, p_{2}, p_{1}^{\prime}, p_{2}^{\prime}\right)$ da forma

$$
\mathcal{I}_{4}=\int \mathcal{D} q \frac{i}{\left(p_{1}^{\prime}-q\right)^{2}} \frac{i}{\left(p_{2}^{\prime}-q\right)^{2}} \Omega_{1}\left(p_{1}^{\prime}-q, p_{2}^{\prime}-q, p_{1}^{\prime}, p_{2}^{\prime}\right)
$$

conforme podemos ver na figura (2.9). O grau de divergência superficial de (2.116) será $d\left(\mathcal{I}_{4}\right)=-2$, ou seja, também finito. Portanto $\mathcal{I}_{4}$ não contribui para a parte divergente de $\Gamma^{(6)}$.

O diagrama $\mathcal{I}_{2}$ pode ser escrito em termos do tensor de polarização $\Pi_{\alpha \beta}$, que pode ser visto na figura (2.1). Assim

O gráfico $\mathcal{G}_{8}$ pode ser escrito em termos de $\Pi^{\mu \nu}$ da forma

$$
\mathcal{I}_{2}\left(p_{1}^{\prime}, p_{2}^{\prime}\right)=\int \mathcal{D} q \frac{i}{\left(p_{1}^{\prime}-q\right)^{2}} \frac{i}{\left(p_{2}^{\prime}+q\right)^{2}}\left(2 p_{1}^{\prime}-q\right)_{\mu}\left(2 p_{2}^{\prime}+q\right)_{\nu} \varepsilon^{\mu \alpha \rho} \frac{q_{\rho}}{q^{2}} \varepsilon^{\nu \beta \gamma} \frac{q_{\gamma}}{q^{2}} \Pi_{\alpha \beta}(q)
$$

que é originalmente logaritmicamente divergente, $d\left(\mathcal{I}_{2}\right)=0$. Porém. substituindo $\Pi_{\alpha \beta}$, equação (2.17), podemos escrerer que

$$
\mathcal{I}_{2}=\frac{1}{16} \int \mathcal{D} q \frac{i}{\left(p_{1}^{\prime}-q\right)^{2}} \frac{i}{\left(p_{2}^{\prime}+q\right)^{2}}\left(2 p_{1}^{\prime}-q\right)_{\mu}\left(2 p_{2}^{\prime}+q\right)_{\nu} \frac{g^{\mu \nu} q^{2}-q^{\mu} q^{\nu}}{q^{2} \sqrt{q^{2}}}
$$

ou ainda

$$
\mathcal{I}_{2}=\frac{1}{4} p_{1 \mu}^{\prime} p_{2 \nu}^{\prime} \int \mathcal{D} q \frac{g^{\mu \nu} q^{2}-q^{\mu} q^{\nu}}{q^{2} \sqrt{q^{2}}\left(p_{1}^{\prime}-q\right)^{2}\left(p_{2}^{\prime}+q\right)^{2}}
$$

que, por contagem de potência, vemos que $d\left(\mathcal{I}_{2}\right)=-2$. Sendo $\mathcal{I}_{2}$ conrergente concluímos que este diagrama não contribui para a parte divergente de $\Gamma^{(6)}$. 

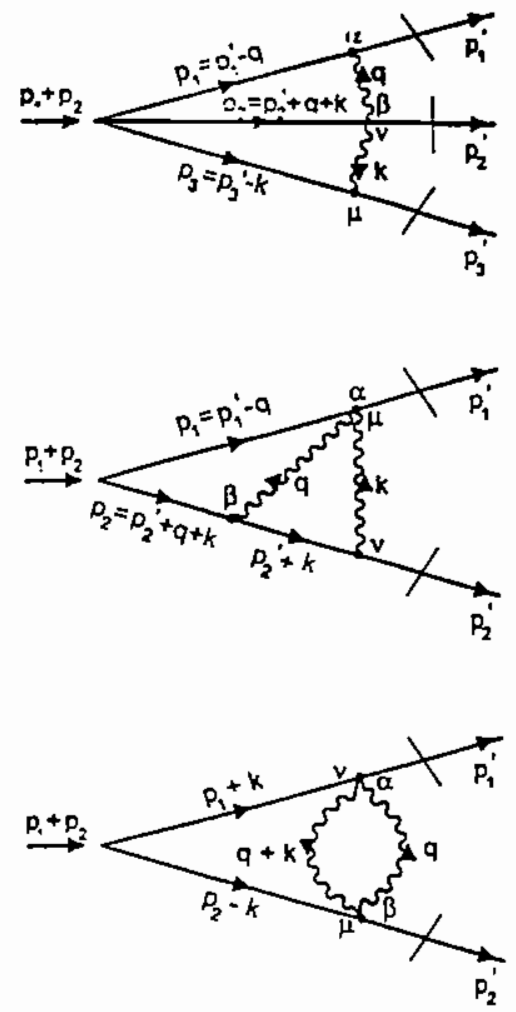

Figura 2.10: Diagramas $\mathcal{I}_{10}, \mathcal{I}_{13}$ e $\mathcal{I}_{14}$.

O último diagrama da figura (2.9), $\mathcal{I}_{6}$ pode ser escrito em termos de $\Theta_{\alpha}\left(p \cdot p^{\prime}\right)$ (ver figura (2.2)), ou seja

$$
\mathcal{I}_{6}=\int \mathcal{D} q \varepsilon^{\alpha \beta_{\rho}} \frac{q_{\rho}}{q^{2}} \frac{i}{\left(p_{1}^{\prime}+q\right)^{2}} \frac{i}{\left(p_{2}^{\prime}-q\right)^{2}}\left(2 p_{2}^{\prime}-q\right)_{\beta} \Theta_{\alpha}\left(p_{1}^{\prime}-q, p_{1}^{\prime}\right)
$$

O grau de divergência superficial de $\Theta_{\alpha}$ é $d\left(\Theta_{\alpha}\right)=-1$. Portanto $d\left(\mathcal{I}_{6}\right)=-2$, ou seja, $\mathcal{I}_{6}$ também é finito. Consequentemente não contribui para a parte divergente de $\Gamma^{(6)}$.

Vamos analisar um outro grupo de diagramas mostrados na figura (2.10).

O gráfico $\mathcal{I}_{10}$ pode ser escrito pode ser escrito como

$$
\begin{aligned}
\mathcal{I}_{10} & =\int \mathcal{D} q \mathcal{D} k \frac{i}{\left(p_{1}^{\prime}-q\right)^{2}} \frac{i}{\left(p_{2}^{\prime}+q+k\right)^{2}} \frac{i}{\left(p_{3}^{\prime}-k\right)^{2}} \\
& \times\left(2 p_{1}^{\prime}-q\right)_{\alpha}\left(2 p_{3}^{\prime}-k\right)_{\mu} g_{\beta \nu} \varepsilon^{\alpha \beta \lambda} \frac{q_{\lambda}}{q^{2}} \xi^{\mu \nu \rho} \frac{k_{\rho}}{k^{2}}
\end{aligned}
$$

que. por contagem de potência é primitivamente logaritimicamente divergente, $d\left(\mathcal{I}_{10}\right)=0$. Porém. após usarmos a propriedade de anti-simetria dos tensores de Levi-Civita a equação (2.121) toma a forma 


$$
\mathcal{I}_{10}=-4 i p_{1 \alpha}^{\prime} p_{3 \mu}^{\prime} \varepsilon^{\alpha \beta \lambda} \varepsilon^{\mu \nu \rho} g_{\beta \nu} \int \mathcal{D} q \mathcal{D} k \frac{q_{\lambda} k_{\rho}}{\left(p_{2}^{\prime}+q+k\right)^{2}\left(p_{1}^{\prime}-q\right)^{2}\left(p_{3}^{\prime}-k\right)^{2}},
$$

que agora apresenta grau de divergência superficial igual a -2 , e concluímos que $\mathcal{I}_{10}$ não contribui para a parte divergente de $\Gamma^{(6)}$.

O mesmo ocorre para o diagrama $\mathcal{I}_{13}$ que primitivamente diverge logaritimicamente, porém, após alguma análise, vê-se que comporta-se como $d\left(\mathcal{I}_{13}\right)=-2$, apresentando uma amplitude finita, e não contruindo para $\Gamma^{(6)}$.

O diagrama $\mathcal{I}_{14}$ pode ser escrito em termos do diagrama $\Theta(p)$, figura (2.2), que representa um "loop" composto de dois propagadores de Chern-Simons. Ou seja,

$$
\mathcal{I}_{14}\left(p_{1}, p_{2}\right)=\int \mathcal{D} k \frac{i}{\left(p_{1}+k\right)^{2}} \frac{i}{\left(p_{2}+k\right)^{2}} \Theta(k) .
$$

Substituindo o resultado para $\Theta(k)$, equação (2.24),

$$
\Theta(k)=-\frac{i}{8} \frac{\left(4 \pi \mu^{2}\right)^{\frac{e}{2}}}{\left[-k^{2}\right]^{-\frac{1}{2}+\frac{e}{2}}}
$$

em (2.123) podemos escrever que

$$
\mathcal{I}_{14}\left(p_{1}, p_{2}\right)=\frac{i\left(4 \pi \mu^{2}\right)^{\frac{\ell}{2}}}{8} \int \mathcal{D} k \frac{1}{\left[-\left(p_{1}+k\right)^{2}\right]\left[-\left(p_{2}+k\right)^{2}\right]\left[-k^{2}\right]^{-\frac{1}{2}+\frac{\varepsilon}{2}}} .
$$

Vamos fazer aqui duas parametrizações. Primeiramente faremos uma parametrização da forma mostrada em (2.90) com $A=-\left(p_{1}+k\right)^{2}$ e $B=-\left(p_{2}+k\right)^{2}$, e em seguida faremos uma segunda parametrização da forma mostrada (2.91), sendo que aqui $A=-k^{2}$ e $B=-\left(p_{1}+k\right)^{2} x-\left(p_{2}-k\right)^{2}(1-z)$. Assim fazendo, temos que

$$
\begin{aligned}
\mathcal{I}_{14}\left(p_{1}, p_{2}\right)= & \frac{i\left(4 \pi \mu^{2}\right)^{\frac{\epsilon}{2}}}{8} \frac{\Gamma\left(3-\frac{d}{2}\right)}{\Gamma(2) \Gamma\left(1-\frac{d}{2}\right)} \int_{0}^{1} d z \int_{0}^{1} d w w^{-\frac{3}{2} \div \frac{\epsilon}{2}} \\
& \int \mathcal{D} k \frac{1}{\left[-k^{2} w-\left(p_{1}+k\right)^{2}(1-w) z-\left(p_{2}-k\right)^{2}(1-w)(1-z)\right]^{\frac{3}{2}+\frac{\epsilon}{2}}} .
\end{aligned}
$$

Lembrando que $\Gamma(3 / 2)=\sqrt{\pi} / 2$ e $\Gamma(-1 / 2)=-2 \sqrt{\pi}$ e arrumando convenientemente o denominador do integrando em $k$ é possível escrever $\mathcal{I}_{14}$ como

$$
\mathcal{I}_{14}\left(p_{1}, p_{2}\right)=\frac{i\left(4 \pi \mu^{2}\right)^{\frac{\ell}{2}} \mu^{\epsilon}}{32 \times 2^{d} \pi^{d}} \int_{0}^{1} d z \int_{0}^{1} d w w^{-\frac{3}{2} \div \frac{\ell}{2}}(1-w) I_{k}
$$


com

$I_{k}=\int d^{d} k \frac{1}{\left\{-k^{2}-2 k \cdot\left[p_{1}(1-w) z-p_{2}(1-w)(1-z)\right]-(1-w) z p_{1}^{2}-(1-w)(1-z) p_{2}^{2}\right\}^{\frac{3}{2}+\frac{\epsilon}{2}}}$.

Usando (B.1), a integral acima tem como resultado

$I_{k}=i \pi^{\frac{d}{2}} \frac{\Gamma(3-d)}{\Gamma\left(3-\frac{d}{2}\right)}\left\{\left[p_{1}(1-w) z-p_{2}(1-w)(1-z)\right]^{2}-(1-w) z p_{1}^{2}-(1-w)(1-z) p_{2}^{2}\right\}^{d-3}$.

Substituindo este resultado em (2.127) e similtaneamente fazendo $d=3(\epsilon \rightarrow 0)$ temos

$$
\mathcal{I}_{14}\left(p_{1}, p_{2}\right)=\frac{1}{32.2^{2} \pi^{2}} \Gamma(\epsilon) \int_{0}^{1} d w w^{-\frac{3}{2}}(1-w)
$$

usando que (ver (B.9))

$$
\int_{0}^{1} d w w^{-\frac{3}{2}}(1-w)=\frac{\Gamma\left(\frac{1}{2}\right) \Gamma(2)}{\Gamma\left(\frac{3}{2}\right)}=-4
$$

Obtemos que $\mathcal{I}_{14}$ é dado por

$$
\mathcal{I}_{14}\left(p_{1}, p_{2}\right)=-\frac{1}{32 \pi^{2}} \frac{1}{\epsilon}+\text { parte finita } .
$$

Vamos agora analisar os diagramas $\mathcal{I}_{15}, \mathcal{I}_{16}, \mathcal{I}_{21}, \mathcal{I}_{22}$ e $\mathcal{I}_{23}$. Estes diagramas, como todos aqueles que compōem a função de seis pontos, são logaritimicamente divergentes. Portanto, como vimos no capítulo I, os contratermos induzidos por estes diagramas serão proporcionais a uma constante. Assim, podemos levar em consideração aqui apenas o primeiro termo da expansão de Taylor dos diagramas, ou seja, analisá-los diretamente para o caso em que os momentos externos são zero. Este caso particular já nos fornecerá, se for o caso, a única colaboração possível para a parte divergente dos diagramas.

Na figura (2.11) estão os diagramas $\mathcal{I}_{15}, \mathcal{I}_{16}, \mathcal{I}_{21}, \mathcal{I}_{22}$ e $\mathcal{I}_{23}$ com momento externo igual a zero.

Para os diagramas $\mathcal{I}_{15}, \mathcal{I}_{16}$, e $\mathcal{I}_{22}$ teremos $\varepsilon^{\mu \nu \lambda} q_{\lambda} q_{\mu}=0$. assim podemos garantir que a parte divergente destes diagramas é nula. Isto se dá sempre que em um vértice trilinear um dos propagadores do campo $\phi$ for um campo externo, e a divergência for logaritimica. Neste caso, o momento externo pode ser tomado como sendo nulo, para que o momento seja conservado no vértice o momento do propagador de $\phi$. restante no vértice derivativo, é igual ao momento do propagador de Chern-Simons.

Para o caso em que o momento externo é igual a zero, podemos dizer que a parte divergente do diagrama $\mathcal{I}_{21}$ é dada por 

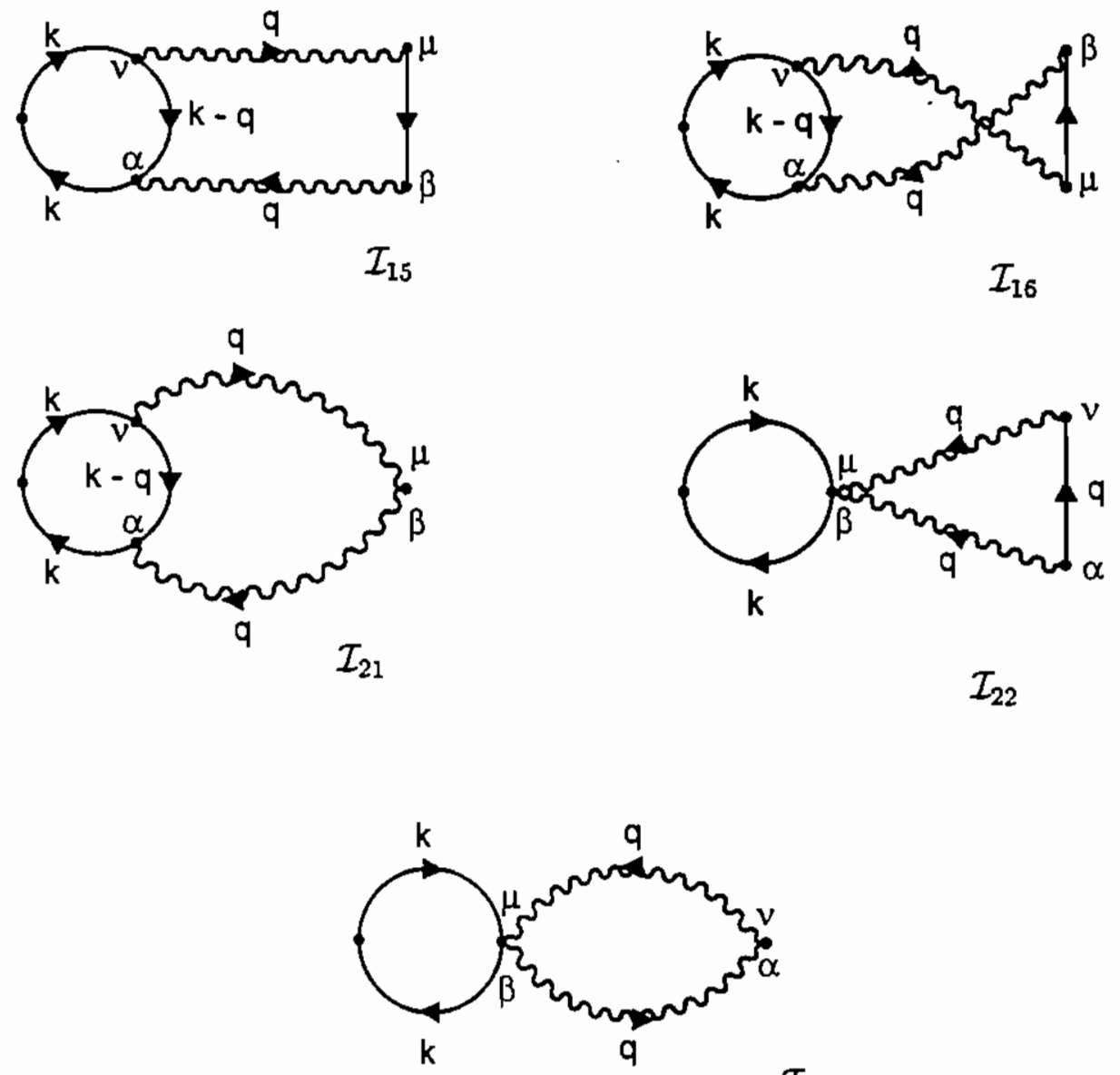

$\mathcal{I}_{23}$

Figura 2.11: Diagramas $\mathcal{I}_{15} . \mathcal{I}_{16}, \mathcal{I}_{21}, \mathcal{I}_{22}$ e $\mathcal{I}_{23}$, todos como momento externo igual a zero. 
Parte divergente de $\mathcal{I}_{21}=-i \int \mathcal{D} k \mathcal{D} q \frac{(2 k+q)_{\nu}(2 k+q)_{\alpha} q_{\lambda} q_{\rho}}{\left[k^{2}\right]^{2}\left[q^{2}\right]^{2}\left[(k+q)^{2}\right]} \varepsilon^{\mu \nu \lambda} \varepsilon^{\alpha \beta \rho} g_{\mu \beta}$,

que pode ainda ser escrita como

Parte divergente de $\mathcal{I}_{21}=\frac{4 i}{(2 \pi)^{2 d}} \varepsilon^{\mu \nu \lambda} \varepsilon^{\alpha \beta \rho} g_{\mu \beta} \mu^{2 \epsilon} \int d^{d} q \frac{q_{\lambda} q_{\rho}}{\left[q^{2}\right]^{2}} \int d^{d} k \frac{k_{\nu} k_{\alpha}}{\left.\left[-k^{2}\right]^{2}\left[-(k+q)^{2}\right]^{2.134}\right)}$

A integral em $k$ acima é da forma (C.3), cuja solução é apresentada em (C.6), e para $\alpha=2$ e $\beta=1$ torna-se

$$
I_{K}=-i \pi^{\frac{d}{2}} \frac{\Gamma\left(2-\frac{d}{2}\right) \Gamma\left(\frac{d}{2}\right) \Gamma\left(2-\frac{d}{2}\right)}{2 \Gamma(d-1)}\left[-q^{2}\right]^{\frac{d}{2}-2}\left[\frac{(4-d)(d-2)}{2\left(\frac{d}{2}-1\right)} \frac{q_{\nu} q_{\alpha}}{\left[-q^{2}\right]}-g_{\nu \alpha}\right] .
$$

Substituindo este resultado em (2.134) obtemos

Parte divergente de $\mathcal{I}_{21}=\frac{2 \pi^{\frac{d}{2}} \mu^{2 \epsilon}}{(2 \pi)^{2 d}} \frac{\Gamma\left(2-\frac{d}{2}\right) \Gamma\left(\frac{d}{2}-1\right)}{2 \Gamma(d-1)} \varepsilon^{\mu \nu \lambda} \varepsilon^{\alpha \beta \rho} g_{\mu \beta} g_{\nu \alpha} \int d^{d} q \frac{q_{\lambda} q_{\rho}}{\left[q^{2}-i \eta\right]^{\left.4-\frac{q^{2}}{2} ., 136\right)}}$

sendo que, para tanto, usamos a propriedade de anti-simetria do tensor de Levi-Civita, anulando assim o primeiro termo do fator entre parênteses de (2.135), e introduzimos o regulador $\eta$ para controlar a divergência infravermelha. A integral em $q$ na equação (2.136), pode ser resolvida usando (B.1). Ao fazermos, temos que

$$
I_{k}=-\frac{2 i \pi g_{\lambda \rho}}{3} \Gamma(\varepsilon)
$$

que, ao ser substituída em (2.136) nos leva ao resultado

$$
\mathcal{I}_{21}=\frac{i}{16 \pi^{2}} \frac{1}{\varepsilon}+\text { partefinita }
$$

Temos ainda o diagrama $\mathcal{I}_{23}$. Pela figura (2.11) vemos que $\mathcal{I}_{23}$ é dado pelo produto direto dos diagramas $\Delta_{2}$ : equação (2.29), e $\Theta(p)$, equação (2.24). ambos finitos, portanto a parte divergente de $\mathcal{I}_{23}$ é nula.

Para obtermos a parte divergente de $\mathcal{J}_{3}$ (logaritimicamente divergente), vamos considerar os momentos externos iguais a zero, (2.12). Baseados na figura (2.15), vemos que, pela anti-simetria do tensor de Levi-Civita,

$$
\text { a parte finita de } \mathcal{J}_{3} \propto \varepsilon^{\mu \nu \rho} q_{\rho}\left(-q_{\nu}\right)=0 \text {. }
$$




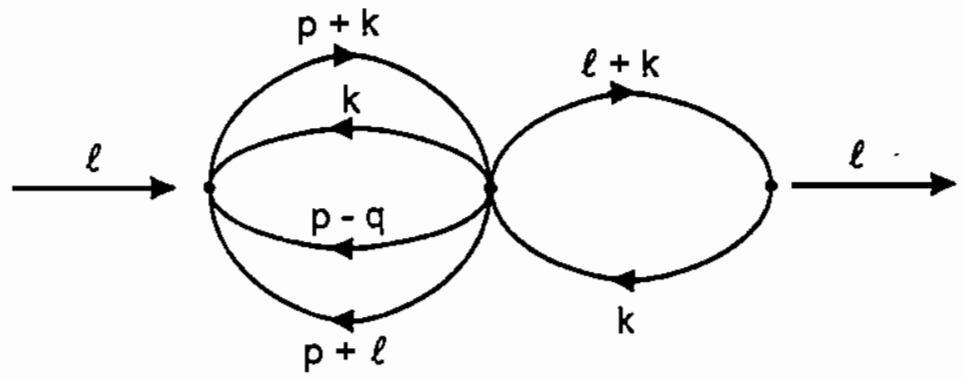

Figura 2.12: Diagrama $\mathcal{J}_{3} \operatorname{com} p_{\text {ext }}=0$.

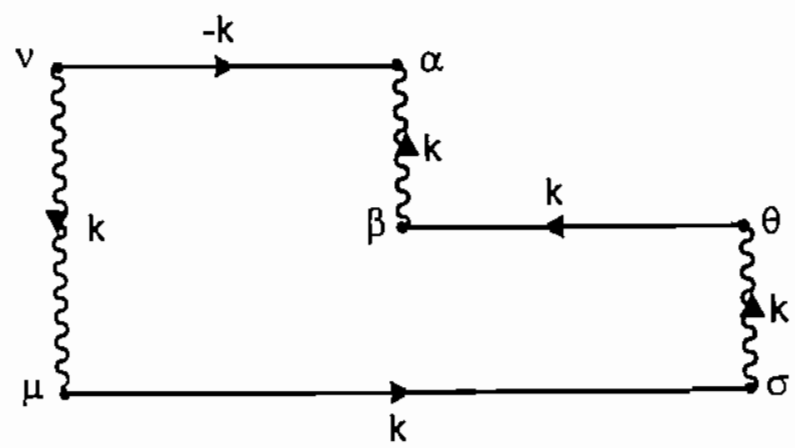

Figura 2.13: Diagrama $\mathcal{K}_{1}$ com $p_{\text {ext }}=0$. 


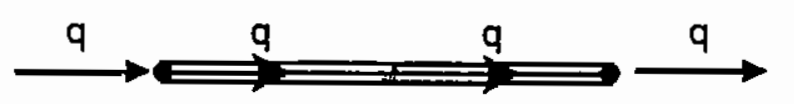

Figura 2.16: Diagrama $\mathcal{L}_{1}$.

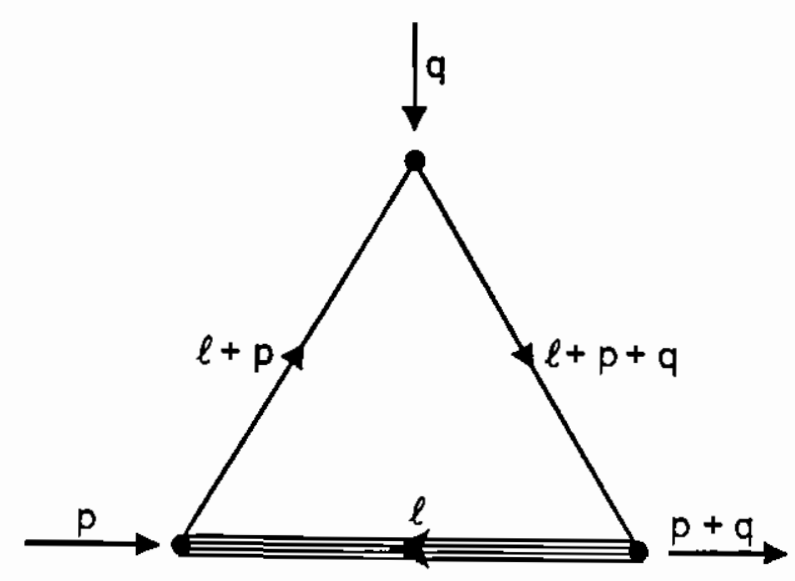

Figura 2.17: Diagrama $\mathcal{L}_{9}$.

temos que

$$
\mathcal{L}_{1}=-\frac{1}{2^{10} \pi^{4}} \frac{1}{\epsilon^{2}}-\frac{2}{2^{10} \pi^{4}}\left[\log \left(\frac{4 \pi \mu^{2}}{-q^{2}+i \eta}\right)+3-\gamma\right] \frac{1}{\epsilon}+\text { parte finita } .
$$

O diagrama $\mathcal{L}_{9}$, mostrado na figura (2.17), pode ser escrito em termos do diagrama $\Delta_{4}$, equação (2.59),

$$
\Delta_{4}(l)=\frac{i}{2^{8} \pi^{2}} \frac{\left(4 \pi \mu^{2}\right)^{\frac{3 \epsilon}{2}}}{\left(-l^{2}+i \eta\right)^{\frac{-1}{2}+\frac{3 \epsilon}{2}}}
$$

Substituindo em (2.144) podemos escrever que

$$
\mathcal{L}_{9}(p, q)=\frac{\left(4 \pi \mu^{2}\right)^{\frac{3 c}{2}}}{2^{8} \pi^{2}} \int \mathcal{D} l \frac{-i}{\left[-(l+p)^{2}\right]} \frac{-i}{\left[-(l+p+q)^{2}\right]} \frac{i}{\left[-l^{2}\right]^{-\frac{1}{2}+\frac{3 i}{2}}} .
$$

Sendo este diagrama logaritimicamente divergente, vamos tomar o momento externo igual a zero para obtermos diretamente a parte divergente. Considerando $p=q=0$. temos que 


$$
\mathcal{L}_{9}(0)=\frac{-i\left(4 \pi \mu^{2}\right)^{\frac{3 \epsilon}{2}}}{2^{8} \pi^{2}} \frac{\mu^{\epsilon}}{2^{d} \pi^{d}} I_{l}
$$

com

$$
I_{l}=\int d^{d} l \frac{1}{\left[-l^{2}+i \eta\right]^{\frac{3}{2}+\frac{3 \epsilon}{2}}} .
$$

cuja solução, usando (B.1), é

$$
I_{l}=i \pi^{\frac{d}{2}} \frac{\Gamma(2 \epsilon)}{\Gamma\left(\frac{3}{2}+\frac{3 \epsilon}{2}\right)}[-\eta]^{-2 \epsilon},
$$

que, substituindo em (2.146), nos dá o resultado

$$
\mathcal{L}_{9}(0)=\frac{1}{2^{11} \pi^{4}} \frac{1}{\epsilon}+\text { parte finita } .
$$

O diagrama $\mathcal{L}_{10}$ pode ser construído a partir de $\Delta_{2}$, equação (2.48). ou, equivalentemente, em termos de $\epsilon$

$$
\Delta_{2}(p)=\frac{-i}{(4 \pi)^{\frac{3}{2}} \sqrt{-p^{2}}}\left(\frac{-4 \pi \mu^{2}}{p^{2}}\right)^{\frac{\epsilon}{2}} \frac{\Gamma\left(\frac{1}{2}-\frac{\epsilon}{2}\right)^{2} \Gamma\left(\frac{1}{2}+\frac{\epsilon}{2}\right)}{\Gamma(1-\epsilon)}
$$

e $\Delta_{3}$, equação (2.53),

$$
\Delta_{3}(q)=\frac{-i}{(4 \pi)^{3}}\left(\frac{4 \pi \mu^{2}}{-q^{2}}\right)^{\epsilon} \frac{\Gamma\left(\frac{1}{2}-\frac{\epsilon}{2}\right)^{3}}{\Gamma\left(\frac{3}{2}-\frac{3 \epsilon}{2}\right)} \Gamma(\epsilon) .
$$

Para este diagrama temos seis fluxos de momentos diferentes no interior do diagrama, segundo o fluxo dos momentos externos. Estes fluxos diferentes são mostrados na figura (2.18) .

Observando os diagramas da figura (2.21) vemos que podemos resumir os três casos em um único que pode ser visto na figura (2.19).

Assim, segundo a figura (2.19) e as equações (2.150) e (2.151), podemos escrever o diagrama $\mathcal{L}_{10}$ como

$$
\mathcal{L}_{10}(p, q)=i \frac{\left(4 \pi \mu^{2}\right)^{\frac{3 \epsilon}{2}}}{(4 \pi)^{\frac{9}{2}}} \frac{\Gamma\left(\frac{1}{2}+\frac{\epsilon}{2}\right) \Gamma\left(\frac{1}{2}-\frac{\epsilon}{2}\right)^{5} \Gamma(\epsilon)}{\Gamma(1-\epsilon) \Gamma\left(\frac{3}{2}-\frac{3 \epsilon}{2}\right)} \int \mathcal{D} k \frac{1}{\left[-(k+p)^{2}\right]\left[-(k+q)^{2}\right]^{\frac{1}{2}+\frac{\epsilon}{2}}\left[-k^{2}\right]^{\epsilon}},
$$



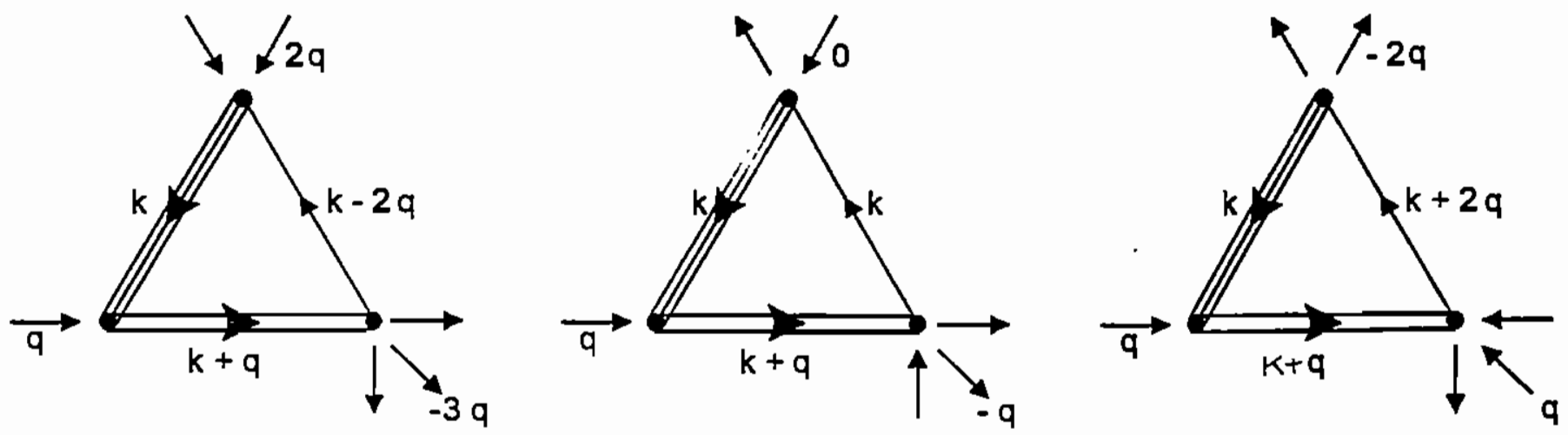

Figura 2.18: Fluxos de momentos possiveis para o diagrama $\mathcal{L}_{10}$.Em cada uma das possibilidades deve ser incluída o caso em que $q \rightarrow-q$.

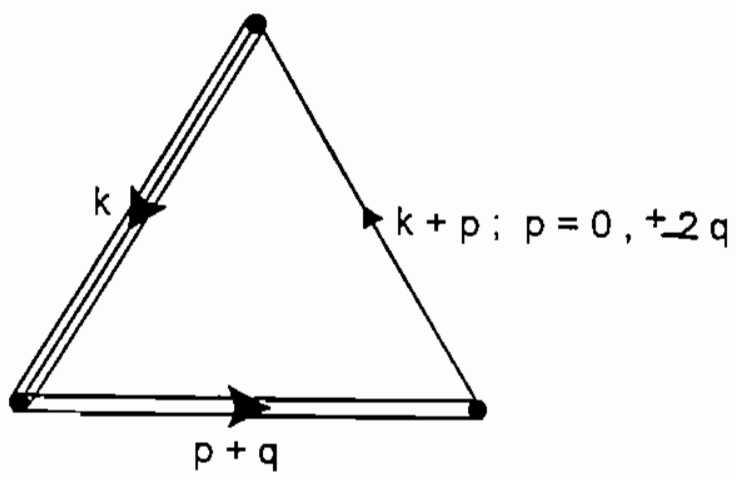

Figura 2.19: Diagrama $\mathcal{L}_{10} \operatorname{com} p=0 . \pm 2 q$. 
ou ainda

$$
\mathcal{L}_{10}(p, q)=C \int d^{d} k \frac{1}{\left[-k^{2}\right]^{\epsilon}}\left\{\frac{1}{\left[-(k+p)^{2}\right]\left[-(k+q)^{2}\right]^{\frac{1}{2}+\frac{\varepsilon}{2}}}\right\}
$$

O termo entre chaves pode ser reescrito usando-se a parametrização (B.7), com $a=$ $-(k+q)^{2}$ e $b=-(k+p)^{2}$. Assim fazendo, temos que

$$
\left.\mathcal{L}_{10}(p, q)=C \frac{\Gamma\left(\frac{3}{2}+\frac{\epsilon}{2}\right)}{\Gamma\left(\frac{1}{2}+\frac{\epsilon}{2}\right)} \int_{0}^{1} d x x^{\frac{1}{2}+\frac{\epsilon}{2}} \int d^{d} k \frac{1}{\left[-k^{2}\right]^{\epsilon}[-(k+q) 2 x-(k+p)(1-x)]^{\frac{(2}{2}+\frac{1}{2}}} 54\right)
$$

Usando novamente a parametrização (B.7), chamando agora $a^{\prime}=-(k+q)^{2} x-(k+p)^{2}(1-$ $x)$ e $b^{\prime}=-k^{2}$, vemos que (2.155) toma a forma

$$
\mathcal{L}_{10}(p, q)=C \frac{\Gamma\left(\frac{3}{2}+\frac{\epsilon}{2}\right)}{\Gamma\left(\frac{1}{2}+\frac{\epsilon}{2}\right) \Gamma(\epsilon)} \int_{0}^{1} d x \int_{0}^{1} d y\left[(1-y)^{\epsilon-1} x^{\frac{\epsilon}{2}-\frac{1}{2}} y^{\frac{\epsilon}{2}+\frac{1}{2}}\right] I_{k}
$$

sendo

$$
I_{k}=\int d^{d} k \frac{1}{\left[-k^{2}-2 k \cdot[q x y+p y(1-x)]-p^{2} y(1-x)-q^{2} x y\right]^{-\frac{3}{2}-\frac{3 c}{2}}} .
$$

A integral acima pode ser resolvida usando (B.1). Assim fazendo, temos que

$$
I_{k}=i \pi^{\frac{d}{2}} \frac{\Gamma(2 \epsilon)}{\Gamma\left(\frac{3}{2}+\frac{3 \epsilon}{2}\right)}\left\{\frac{1}{\left[q^{2} x y(x y-1)+p^{2} y(1-x)[y(1-x)-1]+2 q \cdot p x y^{2}(1-x)\right]^{2 \epsilon}}\right\} .
$$

Substituindo (2.158) em (2.152), podemos escrever

$$
\mathcal{L}_{10}(p, q)=-\frac{1}{(4 \pi)^{6}}\left(\frac{4 \pi \mu^{2}}{q^{2}}\right)^{2 \epsilon} \frac{\Gamma(2 \epsilon) \Gamma\left(\frac{1}{2}-\frac{\epsilon}{2}\right)^{5}}{\Gamma(1-\epsilon) \Gamma\left(\frac{3}{2}-\frac{3 \epsilon}{2}\right)} I\left(\frac{p}{q}, \epsilon\right)
$$

com $I(p / q, \epsilon)$ sendo a integral paramétrica

$$
I\left(\frac{p}{q}, \epsilon\right)=\int_{0}^{1} d x \int_{0}^{1} d y x^{\frac{\epsilon}{2}-\frac{1}{2}} y^{\frac{\epsilon}{2}+\frac{1}{2}}(1-y)^{\epsilon-1} g^{-2 \epsilon}(x, y)
$$

e

$$
g(x, y)=\frac{p^{2}}{q^{2}}\left[-y(1-x)+(1-x)^{2} y^{2}\right]+2 \frac{p \cdot q}{q^{2}} x(1-x) y^{2}+x y(x y-1)
$$


A expressão para $\mathcal{L}_{10}(p, q)$, equação (2.159), apresenta pólo duplo. Um devido ao $\Gamma(2 \epsilon)$, e outro devido a $I(p / q, \epsilon)$, quando $\epsilon \rightarrow 0$ na região de integração próxima de $y=1$.

$$
I\left(\frac{p}{q}, \epsilon\right)_{\epsilon=0}=\int_{0}^{1} d x \int_{0}^{1} d y \frac{x^{\frac{-1}{2}} y^{\frac{1}{2}}}{(1-y)} g^{-2 \epsilon}(x, y) .
$$

Desta forma, devemos calcular os termos divergentes de $\mathcal{L}_{10}(p, q)$ proporcionais a $1 / \epsilon \mathrm{e}$ $1 / \epsilon^{2}$ considerando os dois primeiros termos da expansão de Laurent de $I$. Ou seja, obter os coeficientes $A$ e $B$ de

$$
I=\frac{A}{\epsilon}+B+C \epsilon+\ldots
$$

Para fazer isto vamos primeiramente escrever $I(p / q, \epsilon)$ da forma

$$
I\left(\frac{p}{q}, \epsilon\right)=\int_{0}^{1} d y(1-y)^{\epsilon-1} f(y)
$$

com

$$
f(y)=\int_{0}^{1} d x x^{\frac{e}{2}-\frac{1}{2}} y^{\frac{e}{2}+\frac{1}{2}} g(x, y) .
$$

A equação (2.163) pode, por sua vez, ainda ser expressa pela soma escrevendo

$$
I\left(\frac{p}{q}, \epsilon\right)=I_{1}+I_{2}
$$

com

$$
I_{1}=\int_{0}^{1} d y(1-y)^{\epsilon-1} f(1)
$$

$\mathrm{e}$

$$
I_{2}=\int_{0}^{1} d y(1-y)^{\epsilon-1}[f(x)-f(1)] .
$$

Assim fazendo, vemos que a integral $I_{1}$ tem um pólo simples e admite uma série da forma

$$
I_{1}=\frac{A_{1}}{\epsilon}+B_{1}+C_{1} \epsilon \ldots,
$$

enquanto que em $I_{2}$ o termo de polo é cancelado pela diferença $f(y)-f(1)$ quando $y=1$, deixando $I_{2}$ regular em $\epsilon=0$, admitindo uma série da forma

$$
I_{2}=B_{2}+C_{2} \epsilon+\ldots .
$$


O termo $B_{2}$ é obtido fazendo-se

$$
B_{2}=\left.I_{2}\right|_{\epsilon=0}=4(\ln 2-1),
$$

e a expansão em série de $I_{2}$, equação (2.169), será

$$
I_{2}=4(\ln 2-1)+\mathcal{O}(\epsilon) \text {. }
$$

Para obter os coeficientes da série para $I_{1}$, equação (2.168), devemos calcular (2.164) para $y=1$. Assim fazendo, teremos que

$$
f(1)=(-1)^{-2 \epsilon}\left[\frac{q^{2}}{(p-q)^{2}}\right]^{2 \epsilon} \int_{0}^{1} d x x^{\frac{-3 \epsilon}{2}-\frac{1}{2}}(1-x)^{-2 \epsilon},
$$

ou, usando (B.9),

$$
f(1)=(-1)^{-2 \epsilon}\left[\frac{q^{2}}{(p-q)^{2}}\right]^{2 \epsilon} \frac{\Gamma\left(\frac{1}{2}-\frac{3 \epsilon}{2}\right) \Gamma(1-2 \epsilon)}{\Gamma\left(\frac{3}{2}-\frac{7 \epsilon}{2}\right)} .
$$

Substituindo então em (2.166) e fazendo a integral em $y$ usando novamente (B.9) temos que

$$
I_{1}=(-1)^{-2 \epsilon}\left[\frac{q^{2}}{(p-q)^{2}}\right] \frac{\Gamma\left(\frac{1}{2}-\frac{3 \epsilon}{2}\right) \Gamma(1-2 \epsilon) \Gamma(\epsilon)}{\Gamma\left(\frac{3}{2}-\frac{7 \epsilon}{2}\right) \Gamma(1+\epsilon)} .
$$

Usando (B.10), (B.11), (B.12) e (B.16) podemos escrever que

$$
\begin{aligned}
\Gamma\left(\frac{1}{2}-\frac{3 \epsilon}{2}\right) & =\sqrt{\pi}\left[1+\frac{3 \epsilon}{2}(\gamma+2 \ln 2)\right], \\
\Gamma\left(\frac{3}{2}-\frac{7 \epsilon}{2}\right) & =\sqrt{\pi}\left(\frac{1}{2}-\frac{7 \epsilon}{2}\right)\left[1+\frac{7 \epsilon}{2}(\gamma+2 \ln 2)\right], \\
\Gamma(1-2 \epsilon) & =1+2 \epsilon \gamma+\mathcal{O}\left(\epsilon^{2}\right), \\
\Gamma(1+\epsilon) & =1-\epsilon \gamma+\mathcal{O}\left(\epsilon^{2}\right),
\end{aligned}
$$

substituindo em (2.173), $I_{1}$ terá a forma

$$
I_{1}=\frac{2}{\epsilon}+2\left[2 \ln \left(\frac{-q^{2}}{(q-p)^{2}}\right)-\frac{7 \gamma}{2}+7-4 \ln 2\right]+\mathcal{O}\left(\epsilon^{2}\right)
$$

Para compor $I(p / q, \epsilon)$ devemos somar (2.171) e (2.174), então

$$
I\left(\frac{p}{q}, \epsilon\right)=\frac{2}{\epsilon}+2\left[5+2 \ln \left(\frac{-q^{2}}{(q-p)^{2}}\right)-2 \ln 2-\frac{7 \gamma}{2}\right]+\mathcal{O}\left(\epsilon^{2}\right)
$$




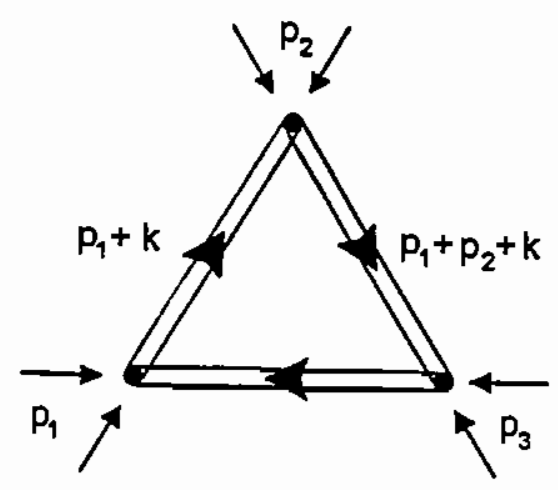

Figura 2.20: Diagrama $\mathcal{L}_{11}$.

Voltando para a equação (2.159) podemos usar (B.10), (B.11), (B.12) e (B.16), para obter as igualdades

$$
\begin{aligned}
\Gamma\left(\frac{1}{2}-\frac{\epsilon}{2}\right) & =\sqrt{\pi}\left[1+\frac{\epsilon}{2}(\gamma+2 \ln 2)\right] \\
\Gamma(1-\epsilon) & =1+\epsilon \gamma+\mathcal{O}\left(\epsilon^{2}\right) \\
\Gamma\left(\frac{3}{2}-\frac{3 \epsilon}{2}\right) & =\frac{\sqrt{\pi}}{2}\left[1-\frac{3 \epsilon}{2}(2-\gamma-2 \ln 2)\right], \\
\Gamma(2 \epsilon) & =\frac{1}{2 \epsilon}-\gamma+2 \epsilon \delta+\mathcal{O}\left(\epsilon^{2}\right)
\end{aligned}
$$

e escrever

$$
\mathcal{L}_{10}(p . q)=-\frac{\pi^{2}}{(4 \pi)^{6}} \frac{1}{\epsilon}\left\{1+\epsilon\left[2 \ln \left(\frac{4 \pi \mu^{2}}{q^{2}}\right)+3+2 \ln 2-2 \gamma\right]\right\} I\left(\frac{p}{q}, \epsilon\right) .
$$

e, por fim, substituindo (2.175) na equação acima, temos como resultado para o diagrama

$$
\mathcal{L}_{10}(p, q)=-\frac{2}{2^{12} \pi^{4}}\left\{\frac{1}{\epsilon^{2}}+\frac{1}{\epsilon}\left[2 \ln \left(\frac{4 \pi \mu^{2}}{-(p-q)^{2}}\right)+8-\frac{11 \gamma}{2}\right]+\mathcal{O}\left(\epsilon^{0}\right)\right\}
$$

sendo que, conforme vimos na figura (2.19), $p=0, \pm 2 q$, na equação acima.

Vamos analisar agora o diagrama $\mathcal{L}_{11}$, mostrado na figura (2.20)

O diagramia $\mathcal{L}_{11}$ pode ser composto por três diagramas $\Delta_{2}$. Assim usando a equação $(2.50)$

$$
\lrcorner_{2}(p)=\frac{-i}{8 \sqrt{-p^{2}}}\left(\frac{-4 \pi \mu^{2}}{p^{2}}\right)^{\frac{2}{2}}
$$

podemos escrerer que

$$
\mathcal{L}_{11}\left(p_{1:} p_{2}\right)=\left(\frac{-i}{8}\right)^{3}\left(4 \pi \mu^{2}\right)^{\frac{3 \epsilon}{2}} \int \mathcal{D} k \frac{1}{\left[-k^{2}\right]^{\frac{1}{2}+\frac{\frac{c}{2}}{2}}\left[-\left(k+p_{1}\right)^{2}\right]^{\frac{1}{2}}\left\{-\left(k-p_{1}+p_{2}\right)^{2}\right]^{\frac{1}{2}+\frac{1}{2}}}
$$


Como este diagrama apresenta divergência logaritimica, podemos considerar o momento externo igual a zero, ou seja

$$
\mathcal{L}_{11}(0)=\left(\frac{-i}{8}\right)^{3}\left(4 \pi \mu^{2}\right)^{\frac{3 \epsilon}{2}} \frac{\mu^{\epsilon}}{2^{d} \pi^{d}} I_{k},
$$

com

$$
I_{k}=\int d^{d} k \frac{1}{\left[-k^{2}+i \eta\right]^{\frac{3}{2}+\frac{3 e}{2}}} .
$$

Usando (B.1), a solução da integral acima é dada por

$$
I_{k}=i \pi^{\frac{d}{2}} \frac{\Gamma(2 \epsilon)}{\Gamma\left(\frac{3}{2}+\frac{3 \epsilon}{2}\right)}[i \eta]^{-2 \epsilon}
$$

a qual, ao ser substituída em (2.180), nos dá o resultado para o diagrama $\mathcal{L}_{11}$

$$
\mathcal{L}_{11}(0)=\frac{-1}{2^{12} \pi^{2}} \frac{1}{\epsilon}+\text { parte finita } .
$$

Feito isto, concluímos o cálculo dos diagramas divergentes que compõem a função de vértice de seis pontos. As amplitudes que apresentam divergências são: $\mathcal{I}_{14}$, equação $(2.132), \mathcal{I}_{21}$, equação $(2.138), \Delta_{3}$, equação $(2.142), \mathcal{L}_{1}$, equação $(2.143), \mathcal{L}_{9}$, equação $(2.149), \mathcal{L}_{10}$, equação $(2.177)$ e $\mathcal{L}_{11}$, equação $(2.182)$, ou seja,

$$
\begin{gathered}
\mathcal{I}_{14}\left(p_{1}, p_{2}\right)=-\frac{1}{32 \pi^{2}} \frac{1}{\epsilon}+\text { parte finita } \\
\mathcal{I}_{21}=\frac{i}{16 \pi^{2}} \frac{1}{\varepsilon}+\text { parte finita } \\
\Delta_{3}(q)=\frac{-i}{32 \pi^{2}}\left[\frac{1}{\epsilon}+\ln \left(\frac{4 \pi \mu^{2}}{-q^{2}+i \eta}\right)+3-\gamma+\mathcal{O}\left(\epsilon^{2}\right)\right], \\
\mathcal{L}_{1}=-\frac{1}{2^{10} \pi^{4}} \frac{1}{\epsilon^{2}}-\frac{2}{2^{10} \pi^{4}}\left[\log \left(\frac{4 \pi \mu^{2}}{-q^{2}+i \eta}\right)+3-\gamma\right] \frac{1}{\epsilon}+\text { parte finita } \\
\mathcal{L}_{9}(0)=\frac{1}{2^{11} \pi^{4}} \frac{1}{\epsilon}\left[\frac{4 \pi \mu^{2}}{i \eta}\right]^{2 \epsilon}+\text { parte finita },
\end{gathered}
$$




$$
\mathcal{L}_{10}(p, q)=-\frac{2}{2^{12} \pi^{4}}\left\{\frac{1}{\epsilon^{2}}+\frac{1}{\epsilon}\left[2 \ln \left(\frac{4 \pi \mu^{2}}{-(p-q)^{2}}\right)+8-\frac{11 \gamma}{2}\right]+\mathcal{O}\left(\epsilon^{0}\right)\right\}
$$

com, nesta última, $q=0,+2 p$ ou $-2 p$, segundo o fluxo dos momentos, $\mathrm{e}$

$$
\mathcal{L}_{11}(0)=\frac{-1}{2^{12} \pi^{2}} \frac{1}{\epsilon}\left[\frac{4 \pi \mu^{2}}{i \eta}\right]^{2 \epsilon}+\text { parte finita } .
$$

Sendo que em cada diagrama deve ser incluído o acoplamento correspondente, conforme tabela (2.2).

$\mathrm{O}$ fator combinatorial depende do fluxo dos momentos em cada diagrama. Assim a figura (2.21) nos mostra as amplitudes que contribuem para a parte divergente da função de vértice de seis pontos, distinguindo os diferentes fluxos de momento, e seus respectivos fatores combinatoriais.

\begin{tabular}{||c|c|c|c||}
\hline Diagramas & Parte Divergente $\left(1 / \epsilon^{2}\right)$ & Parte Divergente $(1 / \epsilon)$ & Acoplamento \\
\hline \hline$\Delta_{3}$ & - & $-\frac{i}{32 \pi^{2}}$ & $\frac{(-i g)^{2}}{2 !(3 !)^{4}} \mu^{2 \epsilon}$ \\
\hline $\mathcal{I}_{14}$ & - & $-\frac{1}{32 \pi^{2}}$ & $\frac{\left(-i e^{2}\right)^{2}}{2 !} \frac{(-i g)}{(3 !)^{2}} \mu^{2 \epsilon}$ \\
\hline $\mathcal{I}_{21}$ & - & $\frac{i}{16 \pi^{2}}$ & $\frac{(-i e)^{2}}{2 !} \frac{i e^{2}}{1 !} \frac{(-i g)}{(3 !)^{2}} \mu^{2 \epsilon}$ \\
\hline $\mathcal{L}_{1}$ & $-\frac{1}{2^{10} \pi^{4}}$ & $\frac{-2}{2^{10} \pi^{4}}\left[\ln \left(\frac{4 \pi \mu^{2}}{g^{2}+i \eta}\right)+3-\gamma\right]$ & $\frac{(-i g)^{3}}{3 !(3 !)^{6}} \mu^{2 \epsilon}$ \\
\hline $\mathcal{L}_{9}$ & - & $\frac{1}{2^{11} \pi^{4}}$ & $\frac{(-i g)^{3}}{3 !(3 !)^{6}} \mu^{2 \epsilon}$ \\
\hline $\mathcal{L}_{10}$ & $-\frac{1}{2^{10} \pi^{4}}$ & $\frac{1}{2^{11} \pi^{4}}\left[2 \ln \left(\frac{4 \pi \mu^{2}}{-(p-q)^{2}+i \eta}\right)+8-\frac{11}{2} \gamma\right]$ & $\frac{(-i g)^{3}}{3 !(3 !)^{6}} \mu^{2 \epsilon}$ \\
\hline $\mathcal{L}_{11}$ & - & $-\frac{1}{2^{12} \pi^{2}}$ & $\frac{(-i g)^{3}}{3 !(3 !)^{6}} \mu^{2 \epsilon}$ \\
\hline
\end{tabular}




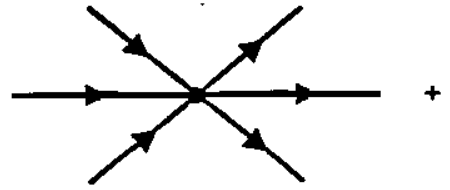

$(3 !)^{2}$

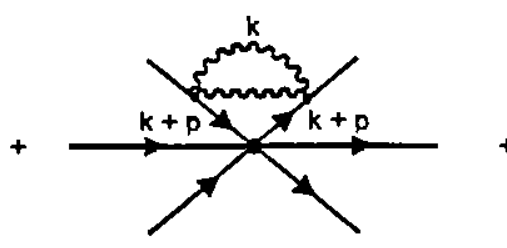

$\left(2^{4} .3^{4}\right) \mathcal{I}_{14}(p, p)$

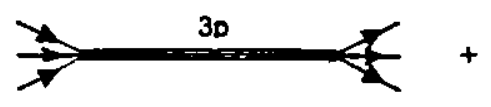

$\left(3^{3} .2^{4}\right) \Delta(3 p)$

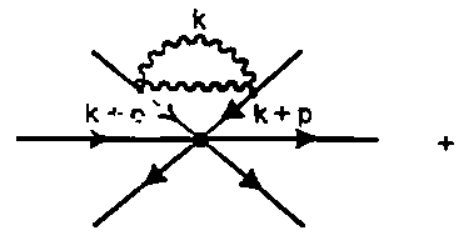

$2\left(2^{4} .3^{3}\right) \mathcal{I}_{14}(p,-p)$

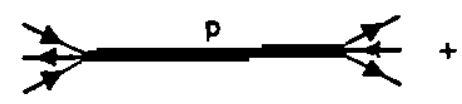

$\left(3^{6} .2^{4}\right) \Delta_{3}(p)$

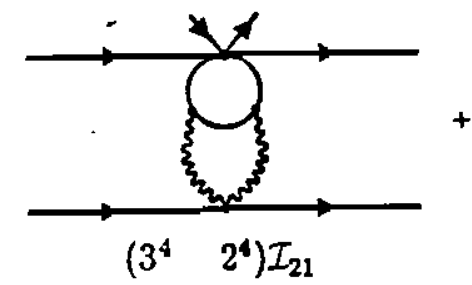

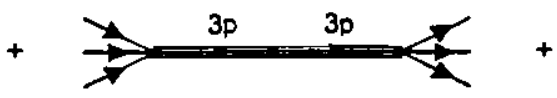

$\left(2^{5} .3^{5}\right) \mathcal{L}_{1}(3 p)$

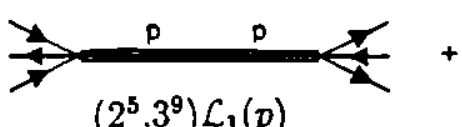

$\left(2^{5} .3^{9}\right) \mathcal{L}_{1}(p)$

$+$

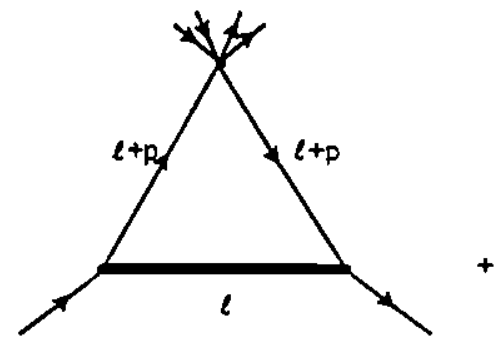

$\left(5.3^{8} .2^{5}\right) \mathcal{L}_{9}(p .0)$

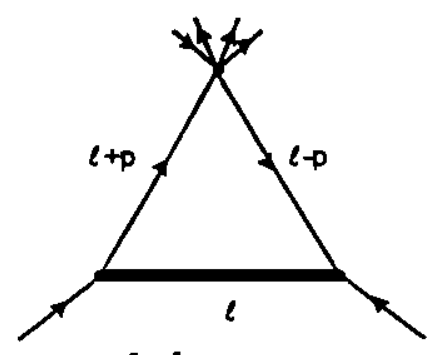

$2\left(3^{8} .2^{5}\right) \mathcal{L}_{9}(p .-2 p)$

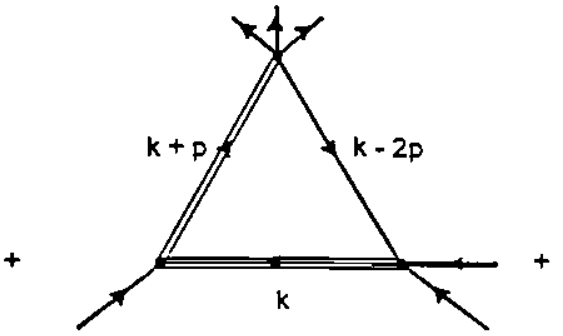

$2\left(3^{8} .2^{5}\right) \mathcal{L}_{10}(-2 p . p)$

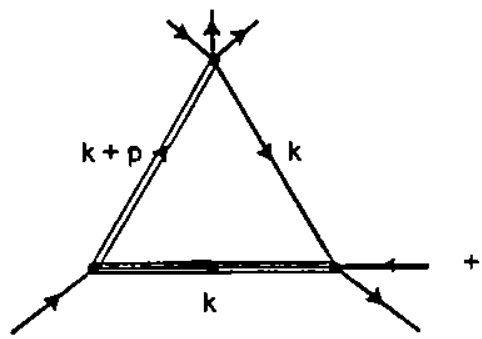

$2\left(3^{10} .2^{6}\right) \mathcal{L}_{10}(0, p)$

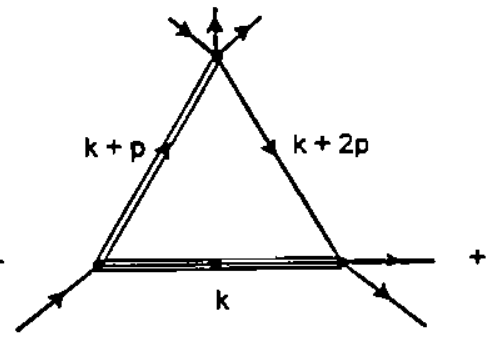

$2\left(7.3^{8} .2^{5}\right) \mathcal{L}_{10}(2 p . p)$

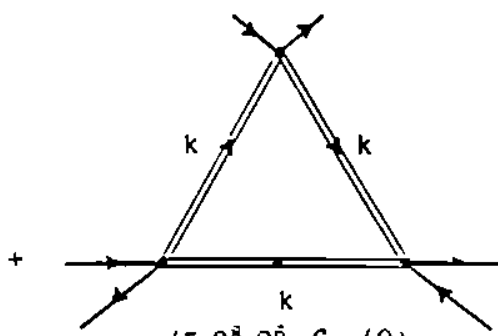

$\left(5.3^{8} \cdot 2^{0}\right) \mathcal{L}_{11}(0)$

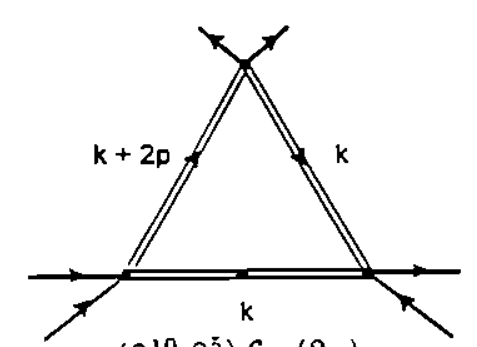

$\left(3^{10} .2^{5}\right) \mathcal{L}_{11}(2 p)$

Figura 2.21: Função de vértice de seis pontos não renormalizada, levando em consideração os diferentes fluxos de momentos 
Tabela 2.2 - Pólo simples, pólo duplo, e acoplamento para as amplitudes que colaboram com a parte divergente da função de seis pontos.

$\mathrm{Na}$ figura (2.21), apenas os diagramas $\Delta_{3}, \mathcal{L}_{1}$ e $\mathcal{L}_{10}$ terão suas amplitudes afetadas pelo fluxo dos momentos, uma vez que a parte divergente destes diagramas dependem do momento através de fatores não locais (logarítimos de $p^{2}$ ). Isto deve-se ao fato de que no cálculo de $\Delta_{3}$ foi levada em consideração sua parte finita, justamente para que os pólos duplos presentes em $\mathcal{L}_{1}$ e $\mathcal{L}_{10}$ (obtidos a partir de $\Delta_{3}$ ) fossem gerados. Os demais diagramas serão afetados pelo fluxo dos momentos apenas no que diz respeito ao fator combinatorial, e não no valor de suas amplitudes, tanto que foram calculados considerando seus momentos externos iguais a zero.

É sabido que os contratermos necessários para renormalizar a teoria devem ser polinomiais[33][50]. Porém, antes de mostrar que os contratermos não locais (logarítimos de $p^{2}$ ) se cancelam, é necessário introduzir a estrutura geral de contratermos para a teoria.

A dificuldade maior na definição da extrutura dos contratermos para este caso é que, além de possuirmos gráficos com divergências globais, temos diagramas que apresentam sub-divergências, chamadas de inclusas ou acavaladas, como vimos ser o caso dos diagramas $\mathcal{L}_{1}$ e $\mathcal{L}_{10}$. A maneira de tratar estas divergências encontra-se na chamada fórmula da floresta[54]. Esta fórmula aplica-se a cada diagrama e nos diz que devemos primeiramente subtrair suas sub-divergências para, em seguida, subtrair a divergência global. Este procedimento é implementado por contratermos adicionados a Lagrangiana, e garante que contratermos não locias não sejam gerados.

Os contratermos necessários para renormalizar a função de seis pontos tem a seguinte estrutura

$$
\Gamma_{C T}^{(6)}=-i g \mu^{2 \epsilon} C_{1}+\frac{(-i g)^{2}}{3} \mu^{2 \epsilon} C_{1} \Delta_{3}(3 p)+3^{2}(-i g)^{2} \mu^{2 \epsilon} C_{1} \Delta_{3}(p)+(-i g) \mu^{2 \epsilon} C_{2},
$$

na qual já estão incluídos os respectivos fatores combinatoriais. Graficamente a equação (2.190) pode ser vista na figura (2.22).

O contratermo $C_{1}$, na equação (2.190) ou nos diagramas $(a),(b)$ e $(c)$ da figura (2.22), é introduzido de maneira a absorver as divergências devidas ao diagrama $\Delta_{3}$, sendo portanto proporcional a $1 / \epsilon$. Desta forma, com a definição do contratermo $C_{1}$ já seremos capazes de fazer cancelar os contratermos não locais, originados de $\Delta_{3}$. O Contratermo $C_{2}$, na equação (2.190) ou no diagrama $(d)$ da figura (2.22), absorverá as divergências restantes, e deverá ter, portanto, fatores proporcionais a $1 \epsilon \mathrm{e} 1 \epsilon^{2}$.

A função de vértice de seis pontos completa, ou seja. incluindo os contratermos, os fatores combinatoriais devidos a cada diagrama (segundo a figura (2.21)), e os acoplamentos mostrados na tabela (2.2) ( $\left.\operatorname{com} e^{2}=\alpha\right)$, será

$$
\Gamma^{(6)}(p)=-i g \mu^{2 \epsilon}\left[1-\frac{i g}{6} \Delta_{3}(3 p)-\frac{i g}{2} \Delta_{3}(p)+C_{1}-18 \alpha^{2} \mathcal{I}_{14}(p, p)-12 \alpha^{2} \mathcal{I}_{14}(p,-p)\right.
$$




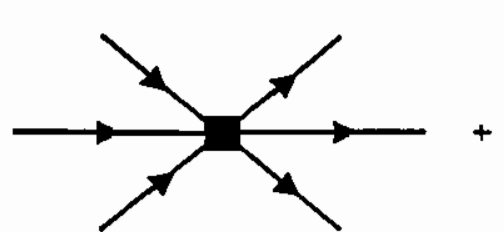

(a)

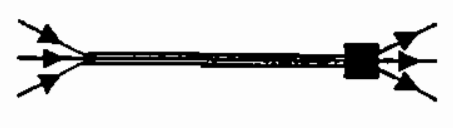

(b) (c)

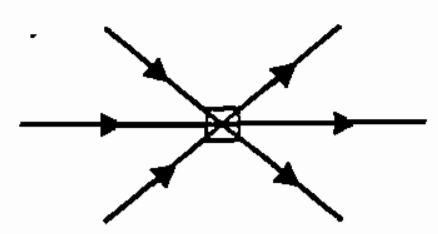

(f)

Figura 2.22: Forma diagramática para os contratermos da função de seis pontos.

$$
\begin{aligned}
& -18 i \alpha^{2} \mathcal{I}_{21}-\frac{g^{2}}{36} \mathcal{L}_{1}(3 p)-\frac{9 g^{2}}{4} \mathcal{L}_{1}(p)-\frac{i g}{3} C_{1} \Delta_{3}(3 p)-9 i g C_{1} \Delta_{3}(p) \\
& -\frac{3 g^{2}}{2} \mathcal{L}_{9}(p:-2 p)-\frac{15 g^{2}}{4} \mathcal{L}_{9}(p, 0)-\frac{3 g^{2}}{2} \mathcal{L}_{10}(-2 p, p)-27 g^{2} \mathcal{L}_{10}(0, p) \\
& \left.-\frac{21 g^{2}}{2} \mathcal{L}_{10}(2 p, p)-\frac{15 g^{2}}{2} \mathcal{L}_{11}(0)-\frac{27 g^{2}}{4} \mathcal{L}_{11}(2 p)+C_{2}\right]
\end{aligned}
$$

Para que $C_{1}$ cancele as divergências devidas a $\Delta_{3}$ na equação anterior, devemos ter

$$
C_{1}=\text { Parte divergente de }\left[\frac{i g}{6} \Delta_{3}(3 p)+\frac{9 i g}{2} \Delta_{3}(p)\right],
$$

que, pela tabela (2.2), podemos escrever

$$
C_{1}=i g\left(\frac{1}{6}+\frac{27}{6}\right)\left(-\frac{i}{32 \pi^{2}} \frac{1}{\epsilon}\right)=g \frac{7}{48 \pi^{2}} \frac{1}{\epsilon} .
$$

Substituindo o valor de $C_{1}$ obtido acima em (2.191), temos

$$
\begin{aligned}
\Gamma^{(6)}(p)= & -i g \mu^{2 \epsilon}\left[1-i g\left(\frac{1}{6} \Delta_{3}(3 p)+\frac{9}{2} \Delta_{3}(p)\right)+\frac{7 g}{48 \pi^{2}} \frac{1}{\epsilon}-a^{2}\left(30 \mathcal{I}_{14}(0)+18 i \mathcal{I}_{21}(0)\right)\right. \\
& -g^{2}\left(\frac{1}{36} \mathcal{L}_{1}(3 p)+\frac{9}{4} \mathcal{L}_{1}(p)\right)-\frac{7 i g^{2}}{48 \pi^{2}} \frac{1}{\epsilon}\left(\frac{1}{3} \Delta_{3}(3 p)+9 \Delta_{3}(p)\right)-\frac{21 g^{2}}{4} \mathcal{L}_{9}(0) \\
& \left.-g^{2}\left(\frac{3}{2} \mathcal{L}_{10}(-2 p, p)+27 \mathcal{L}_{10}(0, p)+\frac{21}{2} \mathcal{L}_{10}(2 p, p)\right)-\frac{57 g^{2}}{4} \mathcal{L}_{11}(0)+C_{2}\right]
\end{aligned}
$$


na qual somamos as amplitudes cujas partes divergentes são pólos simples, para os quais não há influência do fluxo dos momentos, e foram calculadas para $p_{\text {ext }}=0$. Em (2.194) mantivemos explicitamente as amplitudes que apresentam contratermos não locais.

Observe que, por (2.185), (2.186) e (2.188), existem dois tipos de contratermos não locais (logarítimicos em $p^{2}$ ); o primeiro é da forma $\ln \left(\frac{4 \pi \mu^{2}}{-p^{2}+i \eta}\right) \frac{1}{\epsilon}$, devido as amplitudes $\Delta_{3}(p), \mathcal{L}_{1}(p), \mathcal{L}_{10}(0, p)$ e $\mathcal{L}_{10}(2 p, p)$. E o segundo da forma $\ln \left(\frac{4 \pi \mu^{2}}{-9 p^{2}+i \eta}\right) \frac{1}{\epsilon}$, devido a $\Delta_{3}(3 p)$, $\mathcal{L}_{1}(3 p)$ e $\mathcal{L}_{10}(-2 p, p)$. Vamos mostrar que estes termos cancelam-se em (2.194):

- para $\ln \left(\frac{4 \pi \mu^{2}}{-p^{2}+i \eta}\right) \frac{1}{\epsilon}$ temos, na sequência que aparece na equação (2.194), que

$$
\begin{aligned}
& g^{2}\left[-\frac{9}{4}\left(-\frac{2}{2^{10} \pi^{4}}\right)-\frac{7 i}{48 \pi^{2}}\left(-\frac{9 i}{32 \pi^{2}}\right)-27\left(-\frac{1}{2^{11} \pi^{4}}\right)-\frac{21}{2}\left(-\frac{1}{2^{11} \pi^{4}}\right)\right] \frac{1}{\epsilon} \ln \left(\frac{4 \pi \mu^{2}}{-p^{2}+i \eta}\right) \\
= & \frac{g^{2}}{2^{11} \pi^{4} \epsilon} \ln \left(\frac{4 \pi \mu^{2}}{-p^{2}+i \eta}\right)[9-84+75]=0
\end{aligned}
$$

- para $\ln \left(\frac{4 \pi \mu^{2}}{-9 p^{2}+i \eta}\right) \frac{1}{\epsilon}$ temos, na sequência que aparece na equação (2.194), que

$$
\begin{aligned}
& g^{2}\left[-\frac{1}{36}\left(-\frac{2}{2^{10} \pi^{4}}\right)-\frac{7 i}{48 \pi^{2}} \frac{1}{3}\left(-\frac{i}{32 \pi^{2}}\right)-\frac{3}{2}\left(-\frac{2}{2^{11} \pi^{4}}\right)\right] \frac{1}{\epsilon} \ln \left(\frac{4 \pi \mu^{2}}{-9 p^{2}+i \eta}\right) \\
= & \frac{g^{2}}{3^{2} 2^{12} \pi^{4} \epsilon} \ln \left(\frac{4 \pi \mu^{2}}{-9 p^{2}+i \eta}\right)[1-28+27]=0
\end{aligned}
$$

Estas duas últimas igualdades, (2.195) e (2.196), servem como prova de consistência, tanto para assegurarmos quanto ao resultado do cálculo das amplitudes, quanto aos valores obtidos para os fatores combinatoriais.

Uma vez estando seguros que os termos logaritimicos, que dariam origem a contratermos não locais, anulam-se, podemos substituir as amplitudes mostradas de (2.183) até (2.189) em (2.194). Assim, após alguma álgebra, podemos escrever

$$
\Gamma^{(6)}(p)=-i g \mu^{2 \epsilon}\left\{1+\alpha^{2} \frac{33}{16 \pi^{2}} \frac{1}{\epsilon}+g^{2} \frac{582+57 \pi^{2}-1092 \gamma}{2^{14} \pi^{4}} \frac{1}{\epsilon}-g^{2} \frac{49}{2^{8} 3^{2} \pi^{4}} \frac{1}{\epsilon^{2}}+C_{2}\right\}
$$

ou ainda,

$$
C_{2}=-\alpha^{2}\left[\frac{33}{16 \pi^{2}}\right] \frac{1}{\epsilon}-g^{2}\left[\frac{582+57 \pi^{2}-1092 \gamma}{2^{14} \pi^{4}}\right] \frac{1}{\epsilon}+g^{2}\left[\frac{49}{2^{8} 3^{2} \pi^{4}}\right] \frac{1}{\epsilon^{2}}
$$

sendo este o contratermo de ordem dois para a renormalização da função de seis pontos.

Obtidos então os contratermos necessários para renormalizar as funções de vértice de dois e seis pontos, podemos calcular as funções do grupo de renormalização : segundo descritas no capítulo I. Isto será feito no próximo capítulo. 


\section{Capítulo 3}

\section{Análise das Funções do Grupo de Renormalização}

\subsection{Introdução}

Neste capítulo vamos calcular e analisar as funções do grupo de renormalização para o campo base $\phi$, através dos resultados obtidos nos capítulos anteriores. Vamos também analisar a dimensão anômala de alguns operadores compostos.

\subsection{A Função Beta: Pontos Fixos}

Ao estudarmos a equação do grupo de renormalização, vimos que as funções de vértice renormalizadas não mudam com a variação do ponto de renormalização $\mu$. A função beta, definida em (1.71),

$$
\beta_{g}=\mu \frac{\partial g}{\partial \mu}
$$

determina o comportamento da constante de acoplamento como função do parâmetro $\mu$. Observando que de (3.1) podemos escrever que

$$
\int_{g(\mu)}^{g_{r}(\mu, g)} \frac{d g}{\beta(g)}=\ln \mu,
$$

vemos que, quando $\beta(g)=0$, a integral diverge e no lado direito de (3.2) $\mu$ deve tender para infinito ou zero. O comportamento do $\ln \mu$ depende se a integral é positiva ou negativa nas vizinhanças do ponto em que $\beta(g)=0$, ou seja, depende do sinal da função beta nesta região. Os valores de $g$, tais que a função beta anula-se, são chamados de 

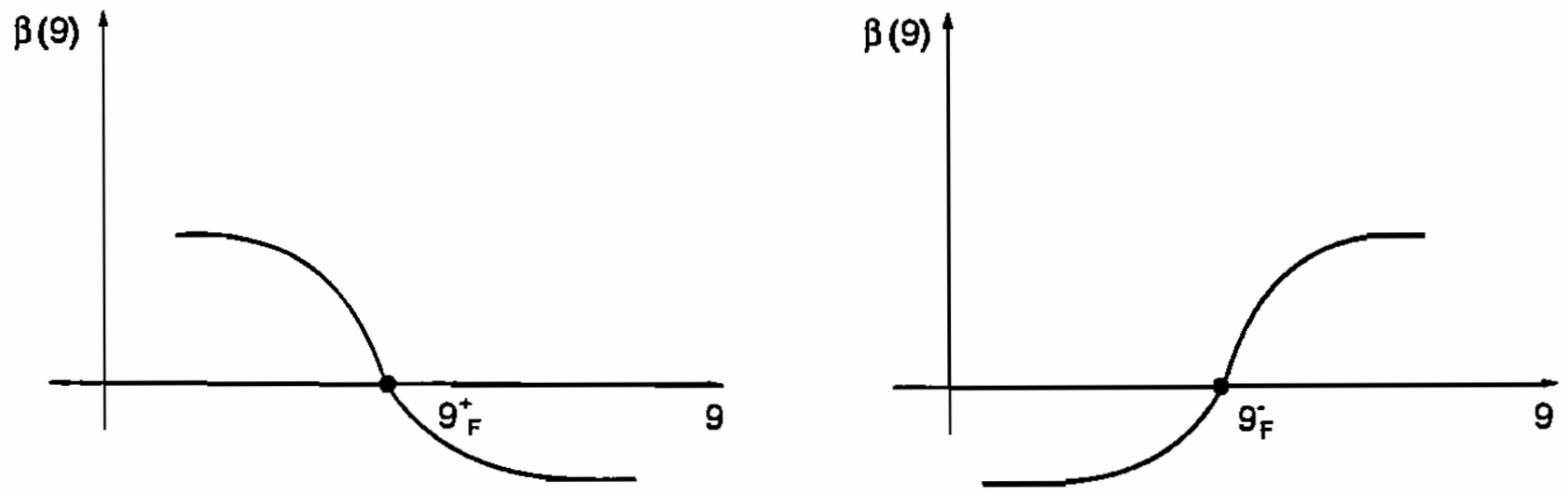

Figura 3.1: Em (a) a inclinação de $\beta(g)$ é negativa e temos um ponto fixo ultravioleta. Em (b) a inclinação de $\beta(g)$ é positiva e temos um ponto fixo infravermelho.

pontos fixos, $g_{F}$. A origem deste nome vem do fato que se a constante de acoplamento tem um valor próximo do ponto fixo, ela tenderá para este ponto quando estivermos na região assintótica $(\mu \rightarrow 0$, ou $\mu \rightarrow \infty)$.

Estas duas situações podem ser analisadas da seguinte maneira: vamos expandir a função beta em uma série de Taylor em torno do ponto fixo, ou seja,

$$
\beta=\mu \frac{\partial g}{\partial \mu}=\left(g-g_{F}\right) \beta^{\prime}\left(g_{F}\right)+\ldots ;
$$

e as duas possibilidades são determinadas pelo sinal de $\beta^{\prime}\left(g_{F}\right)$, conforme a figura (3.1).

No caso (a) da figura (3.1). em que $\beta^{\prime}\left(g_{F}^{+}\right)<0$, temos que se $g$ é menor que $g_{F}^{+}$os sinais negativos se cancelam na expansão de Taylor em (3.3) e $\frac{\partial g}{\partial \mu}$ é positivo, assim quando $\mu$ aumenta $g$ também aumenta: $g$ dirige-se para $g_{F}^{+}$. Se $g$ é menor que $g_{F}^{+}, \frac{\partial g}{\partial \mu}$ é negativo, assim quando $\mu$ aumenta $g$ diminui: $g$ dirige-se novamente para $g_{F}^{+}$. Neste caso $g_{F}^{+}$é dito um ponto fixo ultravioleta estável.

No caso (b) da figura (3.1), em que $\beta^{\prime}\left(g_{F}^{+}\right)>0$, fazendo uma análise análoga a que fizemos no parágrafo anterior. vemos que quando $\mu$ diminui os valores de $g$, tanto à esquerda quanto à direita de $g_{F}^{-}$( $g$ menor e maior que $g_{F}^{-}$respectiramente), tendem para este ponto. Neste caso $g_{F}^{-}$é dito um ponto fixo infravermelho estável.

Resumindo. estas duas situasões podemos dizer simplemente que se

$$
\begin{aligned}
& 3^{\prime}\left(g_{F}\right)<0 \text { : ponto fixo ultravioleta estável. } \\
& \mathcal{B}^{\prime}\left(g_{F}\right)>0 \text { : ponto fixo infravermelho estável. }
\end{aligned}
$$

Pontos fixos ultravioleta estáveis são ditos infravermelhos instáveis (os valores de $g$ afastam-se do ponto fixo quando $\mu \rightarrow 0$ ) e. por conseguinte. pontos infravermelhos estáveis são ultravioletas estáveis. 
No primeiro capítulo vimos que a função Beta é dada pela equação (1.85)

$$
\beta_{g}=\left(\alpha \frac{\partial}{\partial \alpha}+2 g \frac{\partial}{\partial g}-2\right) g_{1}(g, \alpha)
$$

sendo $g_{1}(g, \alpha)$ o resíduo de pólo simples da equaçāo (1.88), dada por

$$
\begin{aligned}
g_{0}(g, \alpha)= & g \mu^{2 \epsilon}\left[1+\left(C_{1}-3 A_{1}\right)+\left(C_{2}-3 A_{2}-3 C_{1} A_{1}\right)+\right. \\
& \left.-\left(3 C_{1} A_{2}+3 C_{2} A_{1}\right)-3 C_{2} A_{2}+\ldots\right]
\end{aligned}
$$

na qual $C_{i}$ e $A_{i}$ são os contratermos necessários para subtrair as divergências das funções de vértice de seis e dois pontos respectivamente, e os índices $i$ e $j$ representam a ordem nas constantes de acoplamento considerada em cada contratermo. A função $g_{1}(g, \alpha)$ em (3.6) é a parte de resíduo de pólo simples da equação (3.7). Do capítulo 2, temos de (2.111), (2.112), (2.193), e (2.198), respectivamente, que

$$
\begin{aligned}
& A_{1}=0 \\
& A_{2}=\left[\frac{7}{48 \pi^{2}} \alpha^{2}-\frac{1}{3^{2} 2^{13} \pi^{4}} g^{2}\right] \frac{1}{\epsilon} \\
& C_{1}=\frac{7}{48 \pi^{2}} g \frac{1}{\epsilon} \\
& C_{2}=-\alpha^{2}\left[\frac{33}{16 \pi^{2}}\right] \frac{1}{\epsilon}-g^{2}\left[\frac{582+57 \pi^{2}-1092 \gamma}{2^{14} \pi^{4}}\right] \frac{1}{\epsilon}+g^{2}\left[\frac{49}{2^{8} 3^{2} \pi^{4}}\right] \frac{1}{\epsilon^{2}}
\end{aligned}
$$

sendo que $\gamma$ é a constante de Euler, $\gamma=0.577$.

Assim, observando a ordem em $1 / \epsilon$ das expressões para $A_{1} . A_{2}, C_{1}$ e $C_{2}$ acima, e baseados em (3.7), vemos que $g_{1}(g, \alpha)$ pode ser escrito como

$$
g_{1}(g, \alpha)=g \mu^{2 \epsilon}\left[\text { Res. de } C_{1}+\text { Res. de pólo simples de } C_{2}-3 \text { Res. de } A_{2}\right] .
$$

Assim, segundo (3.9), (3.10) e (3.11) podemos escrever que

$$
g_{1}(g, \alpha)=a\left[g^{2}-b g^{3}-c \alpha^{2}\right],
$$

sendo $a \approx 0,0147, b \approx 0,0003$ e $c \approx 17,1373$.

Substituindo a expressão (3.13) em (3.6) e operando as derivadas parciais

$$
\beta(g, \alpha)=2 a g\left[g-2 b g^{2}-2 c \alpha^{2}\right] .
$$

A função $\beta(g, \alpha)$ é cúbica em $g$ e quadrática em $\alpha$, e pode ser vista na figura (3.2). 


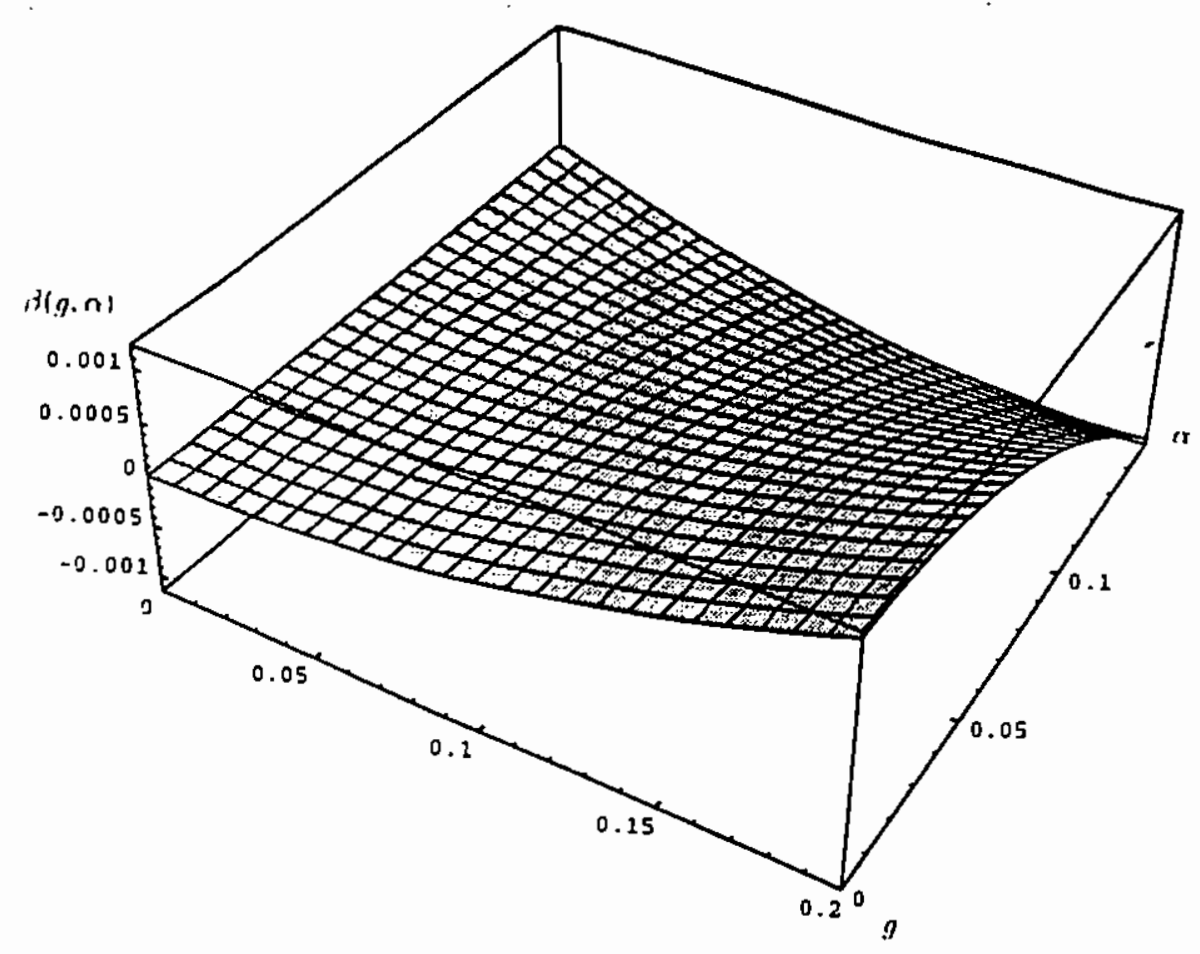

Figura 3.2: Forma da Função $\beta(g, \alpha)$

Para analisar os pontos fixos da teoria, devemos analisar os pontos que anulam a função Beta. Da equação (3.14), podemos ver que existem três valores de $g$ para os quais $\beta(g, \alpha)=0$; o primeiro é o ponto fixo trivial, ou gaussiano,

$$
g=0
$$

que é o ponto fixo na origem. Os outros dois são dados por

$$
g=\frac{1}{4 b}\left[1 \pm \sqrt{1-8 b c \alpha^{2}}\right],
$$

sendo que, para valores pequenos de $\alpha$, o sinal positivo da equação acima nos leva ao resultado $g \simeq\left(1-4 b c \alpha^{2}\right) / 4 b \gg 1$, fora, portanto, da região perturbativa. Já o sinal negativo nos leva ao resultado

$$
g \simeq c \alpha^{2} .
$$

nos indicando, para $c \mathrm{C}^{2} \ll 1$. um condicionamento aos valores de $\alpha$ para os quais tratamento perturbativo tem validade. $\alpha \ll 0,24$. Pela equação anterior vê-se também que existe um conjunto de valores de $g$ e $\alpha$ para os quais a função $\beta(g, \alpha)$ se anula, ou seja. temos uma linha de pontos fixos. A curva descrita pela equação (3.17) pode ser vista na figura (3.3). 


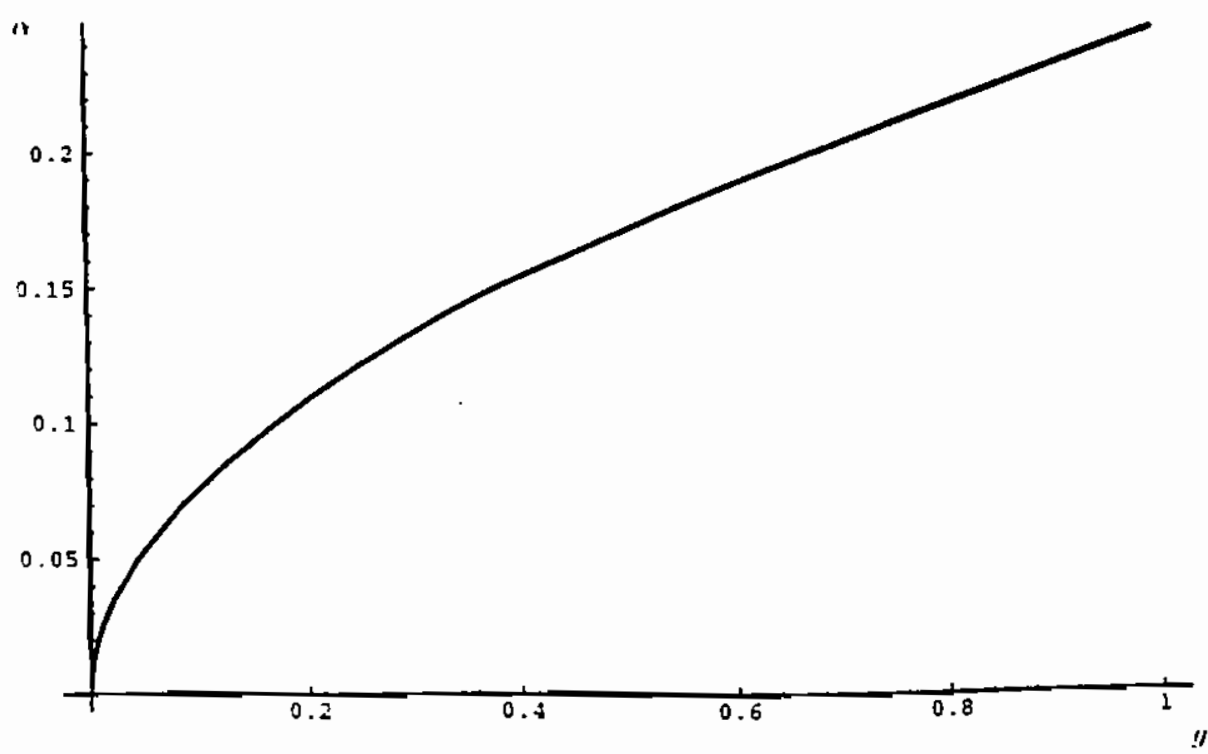

Figura 3.3: Linha de Pontos Fixos $g \simeq c \alpha^{2}, \operatorname{com} c \simeq 17,1373$

Uma vez identificados os valores de $g$ que fazem $\beta(g, \alpha)=0$, vamos analisar a natureza destes pontos fixos. Fazendo a derivada de $\beta(g, \alpha)$ com relação a $g$ temos

$$
\beta^{\prime}(g, \alpha)=4 a g-12 a b g^{2}-2 a c \alpha^{2} .
$$

Para cada ponto fixo temos que

- Para $g=0$

$$
\beta^{\prime}(g, \alpha)=-2 a c \alpha^{2} \rightarrow \beta^{\prime}(g=0,0)<0 .
$$

Portanto, por (3.19), podemos dizer que $g=0$ é um ponto fixo estável ultravioleta. indicando a possibilidade de existência de liberdade assintótica (ou seja, $g \rightarrow 0$ quando $\mu \rightarrow \infty)$.

- Para $g \simeq c \alpha^{2}$

$$
\beta^{\prime}\left(g \simeq c \alpha^{2}, \alpha\right)=2 a c \alpha^{2}\left(1-6 b c \alpha^{2}\right) .
$$

Pode-se ver que para valores de $\alpha$ dentro do intervalo perturbatibo acima definido $\alpha \ll$ $0,24)$, a equação (3.20) é positiva. ou seja

$$
\beta^{\prime}\left(g \simeq c \alpha^{2}, \alpha\right)>0 \quad(\text { para } \alpha \ll 0,24),
$$




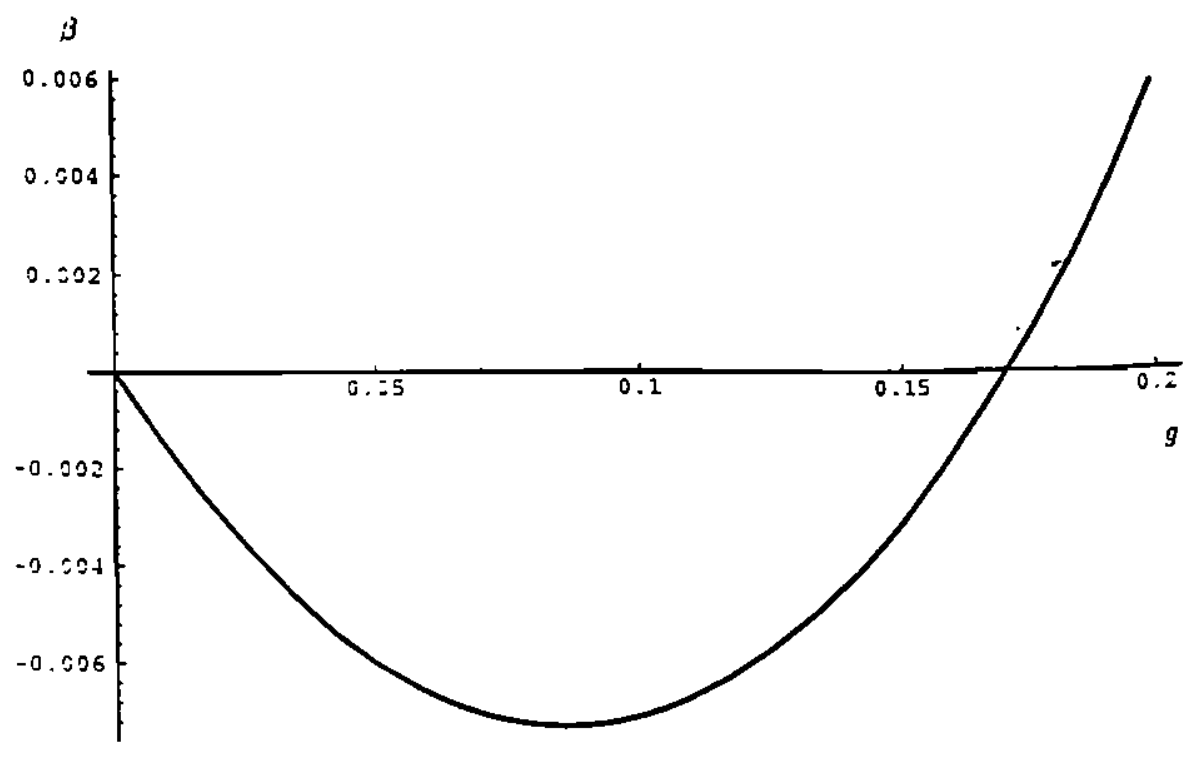

Figura 3.4: Função Beta $(g)$, para $\alpha=0.1$

e. por (3.5), podemos dizer que $g \simeq c \alpha^{2}$ é um ponto fixo estável infravermelho. Este ponto fixo pode ser visto na figura (3.4) para um valor específico de $\alpha, \alpha=0.1$.

Observe ainda que para $\alpha=0$, temos, pela equação (3.17) que $g=0$ também será um zero de beta. Porém, para este caso, $\beta^{\prime}(g=0,0)=0$.

Esta última situação. em que a derivada da função $\beta^{\prime}(g, \alpha)$ não é positiva nem negativa no ponto fixo. necessita uma análise um pouco mais cuidadosa. Para este caso, apesar do sinal da derivada no ponto não estar determinado, vemos que para valores próximos de $g=0$ pela direita $3^{\prime}(g, 0)>0$. Assim, podemos ver que $\left(g-g_{F}\right)<0$, e também $3^{\prime}<0$, ou seja. pela equação (3.3), um aumento de $g$ implica em um aumento de $\mu$, e o ponto fixo $g_{+}=0$ comporta-se como ultravioleta estável. Temos também que para essa região $\left(g-g_{F}\right)<0$, na equação (3.3). Assim, segundo a equação (3.3) vemos que quando $g$ aproxima-se do ponto fixo (diminui) $\mu$ diminui. Portanto podemos concluir que para $a=0$ temos um ponto fixo trivial estável no infravermelho, mudando assim a natureza do ponto fixo trivial, estável no ultravioleta para $\alpha \neq 0$.

Os pontos fixos estáveis da teoria representam os valores efetivos da constante de acoplamento nas regiões assintóticas. Ou seja, se tivermos uma constante de acoplamento com ralor próximo ao ponto fixo. este valor tenderá para o ponto fixo na região assintótica. Portanto: para ralores pequenos de $g$, próximos de zero. teremos que $g \rightarrow 0$ na regiāo ultravioleta quando $\alpha \neq 0$. ou na região infravermelha quando $\alpha=0$. Podemos então garantir que a presença do acoplamento de Chern-Simons faz a teoria de perturbação melhorar para altas energias. Já a ausência deste acoplamento faz a teoria de perturbação 
comportar-se melhor para baixas energias.

\subsection{Análise Dimensional da Equação do Grupo de Renormalização}

Antes de calcularmos a dimensão anômala para o campo $\phi$, é útil analisarmos a dimensionalidade das funções do grupo de renormalização e as relações da dimensão anômala com a invariância de escala.

A equação do grupo de renormalização, equação (1.78), é dada por

$$
\left[\mu \frac{\partial}{\partial \mu}+\beta_{g} \frac{\partial}{\partial g}-n \gamma_{\phi}\right] \Gamma^{(n)}\left(p_{i}, g, \alpha, \mu, \epsilon\right)=0 .
$$

Sendo $\Gamma^{(n)}$ uma função homogênea nas variáveis $p_{i}$ e $\mu$. Então, por uma mudança de escala da forma $\left(p_{i}, g, \alpha, \mu, \epsilon\right) \rightarrow\left(s p_{i}, g, \alpha, s \mu, \epsilon\right)$, a equação (3.22) adimite uma solução da forma

$$
\Gamma^{(n)}\left(s p_{i}, g, \alpha, s \mu, \epsilon\right)=s^{\operatorname{dim} \Gamma^{(n)}} \Gamma^{(n)}\left(p_{i}, g, \alpha, \mu, \epsilon\right)
$$

na qual $s$ é o parâmetro de escala e $\operatorname{dim} \Gamma^{(n)}$ é a dimensão canônica da função de vértice própria de $n$ pontos.

Pelo teorema de Euler para funções homogêneas, podemos dizer que, para a mudança de escala acima, $\Gamma^{(n)}\left(s p_{i}, g: \alpha, s \mu, \epsilon\right)$ deve satisfazer a seguinte equação diferencial

$$
\left[\mu \frac{\partial}{\partial \mu}+s \frac{\partial}{\partial s}\right] \Gamma^{(n)}=\left(\operatorname{dim} \Gamma^{(n)}\right) \Gamma^{(n)}
$$

Usando as equações (3.22) e (3.24) podemos separar em cada uma o termo $\mu \frac{\partial \Gamma^{(n)}}{\partial \mu}$. Igualando estes termos temos que

$$
\left[-s \frac{\partial}{\partial s}+\operatorname{dim} \Gamma^{(n)}+\beta_{g} \frac{\partial}{\partial g}-n \gamma_{\phi}\right] \Gamma^{(n)}=0,
$$

obtendo assim uma equação que relaciona o fator de escala $s$ com uma mudança de $g$. Equivalentemente a forma que definimos as funções Beta no capítulo 1, podemos definir aqui também a função em termos de s, ou seja

$$
s \frac{\partial \bar{g}(g, s)}{\partial s}=\beta(\bar{g})
$$

sendo que $\bar{g}(g, 1)=g$. Explicitando a maneira que $g$ varia com $s$. Cisando esta última definição : a solução da equação (3.25) pode ser escrita na forma[33][49] 


$$
\Gamma^{(n)}\left(s p_{i}, g, \alpha, s \mu, \epsilon\right)=s^{\operatorname{dim} \Gamma^{(n)}} e^{-n \int_{1}^{s} \frac{d x}{x} \gamma_{\phi}(\bar{g}, \alpha, x)} \Gamma^{(n)}\left(p_{i}, \bar{g}(g, s), \alpha, \mu, \epsilon\right) .
$$

Analisando a igualdade acima vemos que se a teoria é livre, $\beta=\gamma=0$, a influência do fator de escala sobre a função de vértice manifesta-se simplesmente através da dimensão canônica de $\Gamma^{(n)}$. Observe que se para algum valor de $\bar{g}$ em que $\beta(\bar{g})=0$, ou seja, um ponto fixo da teoria, a teoria será assintoticamente invariante de escala. A solução (3.27) será então dada por

$$
\Gamma^{(n)}\left(s p_{i}, g, \alpha, s \mu, \epsilon\right)=s^{\operatorname{dim} \Gamma^{(n)}-n \gamma_{\phi}\left(g_{F}, \alpha\right)} \Gamma^{(n)}\left(p_{i}, \bar{g}(g, s), \alpha, \mu, \epsilon\right) .
$$

A dimensão de $\Gamma^{(n)}$ é obtida analisando-se a dimensão da função de Green conexa de $n$ pontos. Pela equação (2.113), temos que

$$
\Gamma^{(n)}=G_{c o n}^{(n)}\left(p_{1}, \ldots, p_{n}\right)\left[\Delta^{(2)}\left(p_{1}\right)^{-1}, \ldots, \Delta^{(2)}\left(p_{n}\right)^{-1}\right],
$$

sendo que $G_{c o n}^{(n)}\left(p_{1}, \ldots, p_{n}\right)$ é a função de Green conexa de $n$ pontos e $\Delta^{(2)}(p)^{-1}$ é o inverso do propagador do campo $\phi$. Dimensionalmente a equação (3.29) nos informa que

$$
\operatorname{dim} \Gamma^{(n)}=\operatorname{dim} G_{c o n}^{(n)}\left(p_{1}, \ldots, p_{n}\right)+2 n .
$$

A função de Green conexa de $n$ pontos no espaço das coordenadas é, por sua vez, definida por

$$
\begin{aligned}
G_{c o n}^{(n)}\left(p_{1}, \ldots, p_{n}\right)(2 \pi)^{d} \delta\left(p_{1}+\ldots+p_{n}\right)= & \int d^{d} x_{1} \ldots d^{d} x_{n} e^{-i \sum_{j} x_{j} p_{j}} \\
& \times<0\left|\hat{T} \phi\left(x_{1}\right) \ldots \phi\left(x_{\frac{n}{2}}\right) \phi^{*}\left(x_{\frac{n}{2}+1}\right) \ldots \phi^{*}\left(x_{n}\right)\right| 0>,
\end{aligned}
$$

que em termos dimensionais pode ser escrita da forma

$$
\operatorname{dim} G_{c o n}^{(n)}-d=n d_{\phi}-n d,
$$

ou ainda, substituindo o valor da dimensão canônica do campo $\phi$, temos

$$
\operatorname{dim} G_{c o n}^{(n)}=-n\left(\frac{d-2}{2}\right)+d,
$$

ou ainda, de (3.30),

$$
\operatorname{dim} \Gamma^{(n)}=d-n d_{\phi} .
$$

Substituindo, então, este último resultado em (3.28) temos por fim que

$$
\Gamma^{(n)}\left(s p_{i}, g, \alpha, s \mu, \epsilon\right)=s^{d-n D_{\phi}} \Gamma^{(n)}\left(p_{i}, g_{F}, a . \mu . \epsilon\right),
$$

sendo $D_{\phi}$ uma "dimensão efetiva" do campo $\phi$,

$$
D_{\phi}=d_{\phi}+\gamma_{\phi},
$$

ou seja, a dimensão anômala $\gamma_{\phi}$ [55] é definida de maneira que soma-se a dimensão canônica de $\phi$, decorrente do processo de renormalização. 


\subsection{Dimensão Anômala do Campo $\phi$}

Com base na equação (1.91), vemos que a dimensão anômala do campo $\phi$ é dada por

$$
\gamma_{\phi}=\left(-\frac{\alpha}{2} \frac{\partial}{\partial \alpha}-g \frac{\partial}{\partial g}\right) Z_{1}(g, \alpha)
$$

sendo que $Z_{1}(g, \alpha)$, observando (1.93), é o termo de ordem $1 / \epsilon$ do contratermo $A$, que renormaliza a função de onda do campo $\phi$. Do capítulo 2, equação (2.112), sabemos que

$$
A=A_{2}=\left[\frac{7}{48 \pi^{2}} \alpha^{2}-\frac{g^{2}}{3^{2} 2^{13} \pi^{4}}\right] \frac{1}{\epsilon} .
$$

Substituindo (3.37) em (3.36) e operando as derivadas parciais, temos que

$$
\gamma_{\phi}=-\frac{7}{48 \pi^{2}} \alpha^{2}+\frac{1}{3^{2} 2^{12} \pi^{4}} g_{F}^{2} .
$$

Portanto, a presença do acoplamento de Chern-Simons atua de maneira a diminuir a dimensão canônica do campo $\phi$, pelo menos no ponto fixo $g_{F}=0$. Este comportamento repercute na dimensão dos vértices da teoria, $d_{a}$, equação (1.23). Podemos escrever que a equação (1.23) comporta-se como

$$
d_{a}=D_{a}+\left(\frac{d-1}{2}\right) \nu_{a}^{A}+D_{\phi} \nu_{a}^{B},
$$

ou seja, observando (3.38) e (3.39) vê-se que o termo de Chern-Simons atua no sentido de diminuir a dimensão de vértice, melhorando sua renormalizabilidade.

Para o ponto fixo $g=g_{+}$(estável no infravermelho), temos que

$$
D_{\phi}=\frac{1}{2}+\bar{a}+\bar{b} \alpha^{2}-\bar{c} \sqrt{\bar{d}+\bar{e} \alpha},
$$

$\operatorname{com} \bar{a}=0,006 \bar{j}, \bar{b}=0,0147, \bar{c}=0,0909, \bar{d}=0,0002$ e $\bar{e}=0,0003$.

\subsection{Operador Composto}

Além de analisar a influência do acoplamento de Chern-Simons sobre a dimensão anômala do campo básico $\phi$, é ilustrativo analisar como dá-se esta influência em alguns operadores compostos. Por operadores compostos entendemos aqueles construídos a partir de polinômios locais do campo básico e suas derivadas no mesmo ponto. No que tange a este trabalho, analizaremos apenas operadores que são polinômios do campo básico. 
Por uma questão de simplicidade, iremos considerar os operadores compostos formalmente integrados, ou seja. operadores culue entram com momento nulo nos diagramas de Feynman.

O grau de divergência superficial para um operador composto $\hat{\mathcal{O}}$ é dada por

$$
d(\hat{\mathcal{O}})=d_{\dot{\mathcal{O}}}-\frac{n}{2},
$$

sendo que $d_{\hat{\mathcal{O}}}$ é a dimensão canônica de $\hat{\mathcal{O}}$ e $n$ o número de campos $\phi$ (ou $\phi^{*}$ ) concorrendo ao mesmo ponto. Neste trabalho vamos iniciar pelos operadores

$$
\begin{array}{cl}
{\left[\phi^{*} \phi\right]} & \rightarrow d_{\phi^{*} \phi}=1 \\
{\left[\left(\phi^{*} \phi\right)^{2}\right]} & \rightarrow d_{\left(\phi^{*} \phi\right)^{2}}=2 \\
{\left[\left(\phi^{*} \phi\right)^{3}\right]} & \rightarrow d_{\left(\phi^{*} \phi\right)^{3}}=3 \\
\cdot & \\
\cdot & \\
\cdot & \\
{\left[\left(\phi^{*} \phi\right)^{n}\right]} & \rightarrow d_{\left(\phi^{*} \phi\right)^{n}}=n .
\end{array}
$$

Em cada um dos exemplos acima nos deteremos no caso que os operadores compostos são ornamentados exclusivamente por campos de Chern-Simons.

- O operador $\left[\phi^{*} \phi\right]$

O operador $\left[\phi^{*} \phi\right]$ tem, usando (3.41), grau de divergência superficial dado por

$$
d\left(\left[\phi^{*} \phi\right]\right)=d_{\left[\phi^{*} \phi\right]}-\frac{n}{2}=\frac{2-n}{2},
$$

ou seja, para $n=2$ temos $d\left(\left[\phi^{*} \phi\right]\right)=0$ (logaritimicamente divergente), e para $n=4$ temos $d\left(\left[\phi^{*} \phi\right]\right)=-1$ (covergente).

O operador $\left[\phi^{*} \phi\right]$ renormalizado é dado por

$$
\left[\dot{\phi}^{*} \dot{\phi}\right]=Z_{\left[\phi^{*} \phi\right]} \dot{\phi}^{*} O=Z_{\left[0^{*} \oplus\right]} Z_{\phi}^{-\frac{n}{2}}\left(\phi_{0}^{*} \phi_{0}\right)=Z_{\phi}^{-1}\left(\phi_{0}^{*} \phi_{0}\right),
$$

uma vez que a divergência apresenta-se para $n=2$. Em termos de contratermos temos que $Z_{\left[\varphi^{-} \rho\right]}=1+A_{\left[0^{*} \oplus\right]}$.

A função de vértice de dois pontos com a inserção do operador composto [ $\left.\phi^{*} \phi \dot{\phi}\right]$ é dada graficamente por

$$
\Gamma^{(2)}\left(\left[\dot{0}^{*} \dot{\phi}\right]\right)=
$$

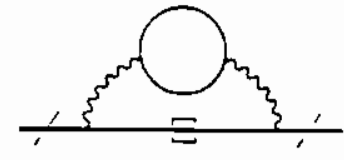

(a)

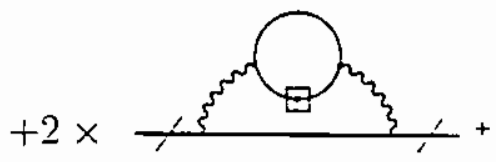

(b) 


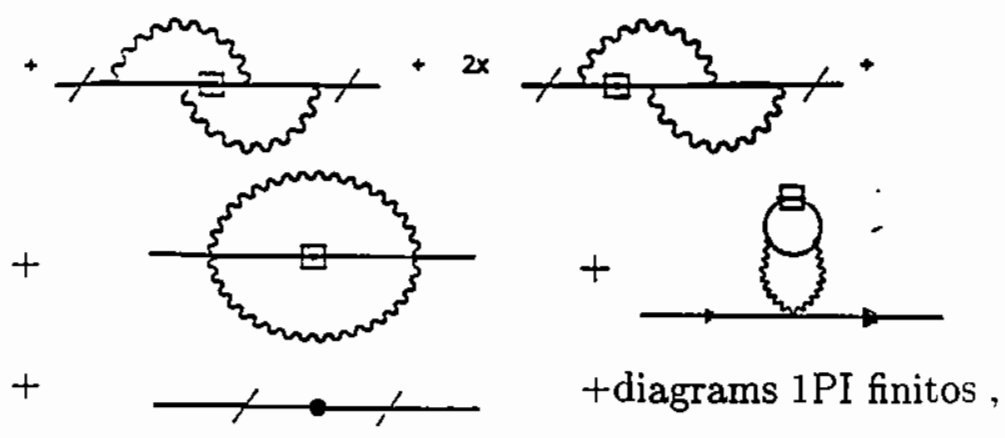

sendo que representa o contratermo necessário para absorver as divergências, e é equivalente a $1+A_{\left[\phi^{*} \phi\right]}$.

Vamos analisar cada um dos diagramas da expressão (3.45). Para tanto, vamos nos basear na figura (2.7) e na equação (2.72) para o diagrama $\mathcal{C}_{1}$. $A$ diferença é que para o caso da inserção do operador composto devemos tomar o quadrado do propagador do campo $\phi$ em (2.72), ou seja

$$
(a)=\int \mathcal{D} k \frac{1}{\left[(p+k)^{2}\right]^{2}}(2 p+k)_{\mu}(2 p+k)_{\nu} \varepsilon^{\mu \alpha \rho} \frac{k_{\rho}}{k^{2}} \Pi_{\alpha \beta} \epsilon^{\beta \nu \sigma} \frac{k_{\sigma}}{k^{2}} .
$$

que, como trata-se de um diagrama logaritimicamente divergente, podemos escrever a equação acima para $p_{\text {ext }}=0$ e assim obtermos diretamente a parte divergente. Assim,

$$
(a)\left(p_{e x t}=0\right)=\int \mathcal{D} k \frac{k_{\mu} k_{\nu} k_{\rho} k_{\sigma}}{\left[(k+i \eta)^{2}\right]^{2}\left[k^{2}\right]^{2}} \varepsilon^{\mu \alpha \rho} \Pi_{\alpha \beta} \varepsilon^{\beta \nu \sigma}=0,
$$

ou seja. a parte divergente de $(a)$ é nula.

O mesmo ocorre com a parte divergente dos demais diagramas da equacão (3.45), exceto para $(e)$ e $(f)$. Esta análise é equivalente àquela que fizemos para os diagramas da função de vértice de seis pontos (também logaritimicamente divergentes). nos quais um dos propagadores do campo $\phi$ de um vértice tri-linear é um propagador externo.

A função de vértice de dois pontos reduz-se então a

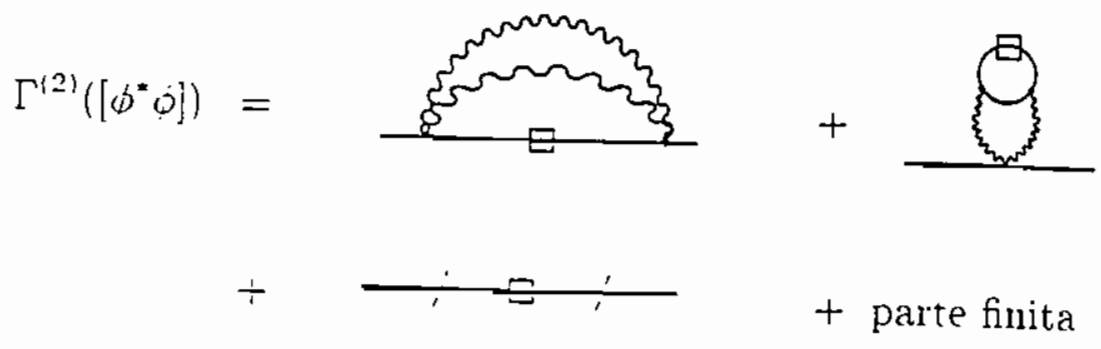

ou ainda. pelos diagramas $\mathcal{I}_{11}$ e $\mathcal{I}_{21}$. ilustrados. respectivamente nas figuras $(2.10)$ e (2.11). temos que 


$$
\Gamma^{(2)}\left(\left[\phi^{*} \phi\right]\right)=\frac{(-i e)^{2}}{2 !}\left(2^{2}\right) \mathcal{I}_{14}\left(p_{1},-p\right)+\frac{(-i e)^{2}}{2 !} \frac{i e^{2}}{1 !}\left(2^{2}\right) \mathcal{I}_{21}+\left(1+A_{\left[\phi^{*} \phi\right]}\right)+\text { parte finita }
$$

sendo que nos dois primeiros termos do lado esquerdo da equação acima temos, respectivamente, o acoplamento, o fator combinatorial e a amplitude referente a cada diagrama.

Usando em (3.49) os resultados para $\mathcal{I}_{14}$ e $\mathcal{I}_{21}$ mostrado nas equações (2.132) e (2.138), temos

$$
\Gamma^{(2)}\left(\left[\phi^{*} \phi\right]\right)=\frac{3}{16 \pi^{2}} \alpha^{2} \frac{1}{\epsilon}+A_{\left[\phi^{*} \phi\right]}+\text { parte finita } .
$$

Sendo $\left.A_{\left[\phi^{*} \phi\right]}\right)$ o contratermo que deixa finita a função de vértice $\Gamma^{(2)}\left(\left[\phi^{*} \phi\right]\right)$, então temos que

$$
A_{\left[\phi^{*} \phi\right]}=-\frac{3}{16 \pi^{2}} \alpha^{2} \frac{1}{\epsilon}
$$

- O operador $\left[\left(\phi^{*} \phi\right)^{2}\right]$

O operador composto $\left[\left(\phi^{*} \phi\right)^{2}\right]$ tem, usando (3.41), grau de divergência superficial igual a

$$
d\left[\left(\phi^{*} \phi\right)^{2}\right]=d_{\left[\left(\phi^{*} \phi\right)^{2}\right]}-\frac{n}{2}=\frac{4-n}{2}
$$

ou seja,

$$
\begin{aligned}
& \text { para } n=2 \rightarrow d\left[\left(\phi^{*} \phi\right)^{2}\right]=1, \text { Linearmente divergente } \\
& \text { para } n=4 \rightarrow d\left[\left(\phi^{*} \phi\right)^{2}\right]=0, \text { Logaritimicamente divergente } \\
& \text { para } n>4 \rightarrow \text { finito. }
\end{aligned}
$$

O contratermo que renormaliza o operador composto teria, em princípio, uma estrutura da forma $A_{\left[\left(\phi^{*} \phi\right)^{2}\right]}+A_{\left[\left(\phi^{*} \phi\right)^{2}\right]}^{\prime} \sqrt{p^{2}}$. Porém, o termo multiplicativo de $\left.A_{[}^{\prime}\left(\phi^{*} \phi\right)^{2}\right]$ não é polinomial, e o teorema de Weinberg[50] nos garante que o contratermo será apenas uma constante. Isto nos garante também que não há termos de mistura para este operador. Os termos de mistura com outros operadores compostos (de dimensão menor ou igual a dimensão canônica de $\left.\left[\left(\phi^{*} \phi\right)^{2}\right]\right)$, viriam de operadores de dois pontos, como por exemplo $\left[\left(\phi^{*} \phi\right)\right]$ ou $\left[\left(\phi^{*} \partial^{2} \phi\right)\right]$. Como vimos o teorema de Weinberg nos garante que não existe renormalização associada a este tipo de função de vértice. 
O operador $\left[\left(\phi^{*} \phi\right)^{2}\right]$ renormalizado é dado por

$$
\left[\left(\phi^{*} \phi\right)^{2}\right]=Z_{\left[\left(\phi^{*} \phi\right)^{2}\right]}=Z_{\left[\left(\phi^{*} \phi\right)^{2}\right]} Z_{o}^{-\frac{n}{2}}\left(\phi_{0}^{*} \phi_{0}\right)^{2}=Z_{\left[\left(\phi^{*} \phi\right)^{2}\right]} Z_{\phi}^{-2}\left(\phi_{0}^{*} \phi_{0}\right)^{2},
$$

uma vez que a divergência apresenta-se em $n=4$. Em termos de contratermos temos que $Z_{\left[\left(\phi^{*} \phi\right)^{2}\right]}=1+A_{\left[\left(\phi^{*} \phi\right)^{2}\right]}$.

A função de vértice de quatro pontos para o operador $\left[\left(\phi^{*} \phi\right)^{2}\right]$ terá a seguinte forma diagramática

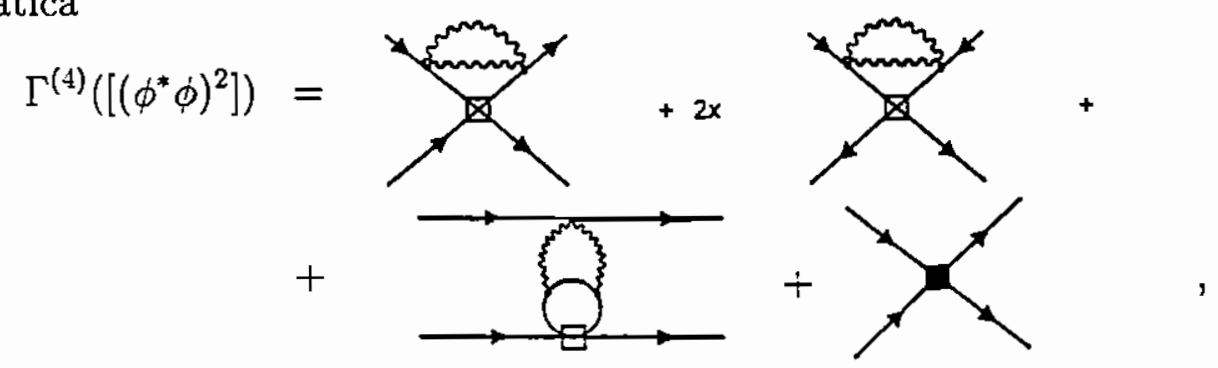

sendo que o último diagrama do lado esquerdo representa o contratermo $\left(1+A_{\left[\left(\phi^{*} \phi\right)^{2}\right]}\right)$. Observe ainda que consideramos para a função de quatro pontos apenas ornamentações com Chern-Simons ao operador composto(que ocupa o lugar do que seria o vértice de interação dos campos $\phi)$. Para escrevermos a equação (3.54) nos baseamos na análise feita no capítulo 2, quando discutimos a função de seis pontos. Nesta ocasião vimos que os únicos diagramas que colaboram para a parte divergente da função de vértice são os diagramas $\mathcal{I}_{14}$ e $\mathcal{I}_{21}$.

Explicitando os acoplamentos e os fatores combinatoriais em (3.54) podemos escrever

$$
\begin{aligned}
\Gamma^{(4)}\left(\left[\left(\phi^{*} \phi\right)^{2}\right]\right) & =\frac{\left(-i e^{2}\right)^{2}}{2 !}\left[\left(2^{6}\right) \mathcal{I}_{14}\left(p_{1},-p\right)+\left(2 \times 2^{4}\right) \mathcal{I}_{14}\left(p_{1} p\right)\right] \\
& +\frac{(-i e)^{2}}{2 !} \frac{i e^{2}}{1 !}\left(2^{6}\right) \mathcal{I}_{21}+4\left(1 \div A_{\left[\left(\phi^{*} \phi\right)^{2}\right]}\right)+\text { parte finita }
\end{aligned}
$$

Substituindo acima os valores de $\mathcal{I}_{14}$, equação (2.132). e $\mathcal{I}_{21}$, equação (2.138), temos que

$$
\Gamma^{(4)}\left(\left[\left(\phi^{*} \dot{\phi}\right)^{2}\right]\right)=\frac{\tau}{2 \pi^{2}} \alpha^{2} \frac{1}{\epsilon}+4\left(1+A_{\left[\left(0^{*} \circ\right)^{2} \cdot\right)}\right)+\text { parte finita } .
$$

Da qual podemos escrever que o contratermo $A_{\left\{\left(\phi^{-} \delta\right)^{2}\right.}$ é dado por

$$
A_{\left[\left(\varphi^{*} \phi\right)^{2}\right]}=-\frac{7}{8 \pi^{2}} \alpha^{2} \frac{1}{\epsilon}
$$

- O operador $\left[\left(\phi^{*} \phi\right)^{3}\right]$ 
O grau de divergência superficial para este operador é dado por (ver (3.41)

$$
d\left[\left(\phi^{*} \phi\right)^{3}\right]=d_{\left(\phi^{*} \phi\right)^{3}}-\frac{n}{2}=\frac{6-n}{2}
$$

ou seja

$$
\begin{aligned}
& \text { para } n=2 \rightarrow d\left[\left(\phi^{*} \phi\right)^{3}\right]=2, \text { Quadraticamente divergente } \\
& \text { para } n=4 \rightarrow d\left[\left(\phi^{*} \phi\right)^{3}\right]=1, \text { Linearmente divergente } \\
& \text { para } n=6 \rightarrow d\left[\left(\phi^{*} \phi\right)^{3}\right]=0, \text { Logaritimicamente divergente } \\
& \text { para } n>6 \rightarrow \text { finito. }
\end{aligned}
$$

Para $n=2$ os contratermos teriam a forma $A_{\left[\left(\phi^{*} \phi\right)^{3}\right]}^{\prime} p^{2}$. Porém todos os diagramas de dois pontos que contém o vértice especial $\left[\left(\phi^{*} \phi\right)^{3}\right]$ ornamentado com o campo de ChernSimons são nulos. Isto pode ser visto procedendo a análise de maneira análoga a que fizemos no capítulo 2, ao estudarmos a função de dois pontos. Para $n=4$ o contratermo seria polinomial, da forma $A_{\left[\left(\phi^{*} \phi\right)^{3}\right]} \sqrt{p^{2}}$. Portanto também não temos termos de mistura.

O operador $\left[\left(\phi^{*} \phi\right)^{3}\right]$ renormalizado é dado por

$$
\left[\left(\phi^{*} \phi\right)^{3}\right]=Z_{\left[\left(\phi^{*} \phi\right)^{3}\right]}\left(\phi^{*} \phi\right)^{3}=Z_{\left[\left(\phi^{*} \phi\right)^{3}\right]} Z_{\phi}^{-\frac{n}{2}}\left(\phi_{0}^{*} \phi_{0}\right)^{2}=Z_{\left[\left(\phi^{*} \phi\right)^{3}\right]} Z_{\phi}^{-3}\left(\phi_{0}^{*} \phi_{0}\right)^{3},
$$

uma vez que a divergência apresenta-se em $n=6$. Em termos de contratermos temos que $Z_{\left[\left(\phi^{*} \phi\right)^{3}\right]}=1+. A_{\left[\left(\phi^{*} \phi\right)^{3}\right]}$.

A função de vértice de seis pontos para o operador $\left[\left(\phi^{*} \phi\right)^{3}\right]$ é dada por

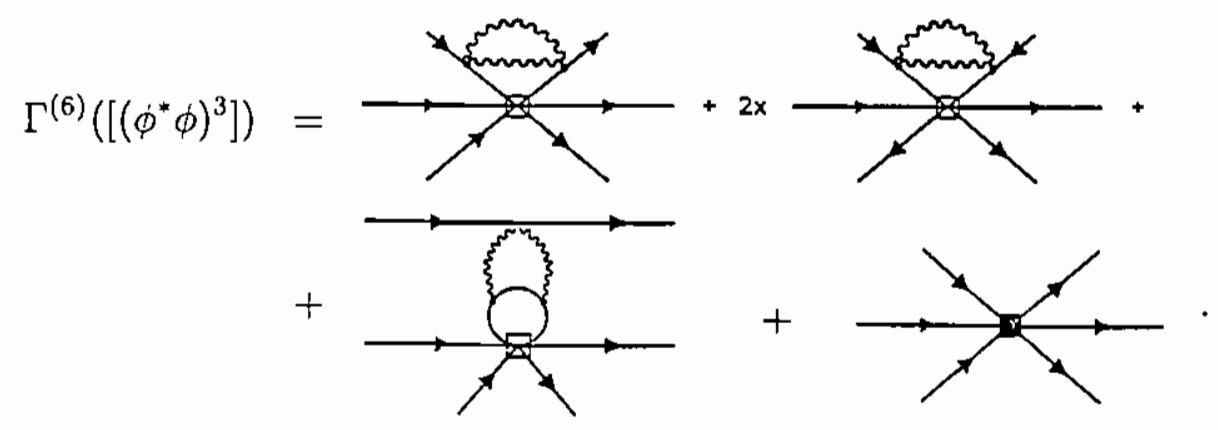

sendo que o último diagrama da equação acima é o contratermo.

A equação (3.60) pode ainda. explicitando os acoplamentos e os fatores combinatoriais, ser escrita como

$$
\begin{aligned}
\Gamma^{(6)}\left(\left[\left(\phi^{*} \phi\right)^{3}\right]\right) & =\frac{\left(i e^{2}\right)^{2}}{2 !}\left[\left(3^{4} \times 2^{4}\right) \mathcal{I}_{14}\left(p_{1},-p\right)+2\left(3^{3} \times 2^{4}\right) \mathcal{I}_{14}\left(p_{1}, p\right)\right] \\
& +\frac{(-i e)^{2}}{2 !} \frac{i e^{2}}{1 !}\left(3^{-4}+2^{4}\right) \mathcal{I}_{21}+\left(3^{2} \times 2^{2}\right)\left(1+. A_{\left[\left(\phi^{*} \phi\right)^{3}\right]}\right)+\text { parte finita }
\end{aligned}
$$


substituindo o valor para $\mathcal{I}_{14}$, equação $(2.132)$, e $\mathcal{I}_{21}$, equação (2.138) temos que a função de vértice édada por

$$
\Gamma^{(6)}\left(\left[\left(\phi^{*} \phi\right)^{3}\right]\right)=\frac{3^{3} \times 11}{4 \pi^{2}} \alpha^{2} \frac{1}{\epsilon}+\text { parte finita } .
$$

E o contratermo $A_{\left[\left(\phi^{*} \phi\right)^{3}\right]}$ será,

$$
A_{\left[\left(\phi^{*} \phi\right)^{3}\right]}=-\frac{33}{16 \pi^{2}} \alpha^{2} \frac{1}{\epsilon},
$$

que, como era esperado, é igual ao contratermo de ordem $\alpha^{2}$ que absorve a divergência da função de vértice de seis pontos para o campo $\phi$, equação (2.198).

Repetindo o procedimento até aqui desenvolvido, podemos escrever diretamente que contratermo que absorve as divergências do operador composto $\left[\left(\phi^{*} \phi\right)^{4}\right]$ é dado por

$$
A_{\left[\left(\phi^{*} \phi\right)^{4}\right]}=-\frac{15}{4 \pi^{2}} \alpha^{2} \frac{1}{\epsilon} .
$$

Estes resultados podem ser generalizados para $2 n$ campos externos. A função de vértice de $2 n$ campos para o operador composto $\left[\left(\phi^{*} \phi\right)^{n}\right]$ é dada por

$$
\begin{aligned}
\Gamma^{(2 n)}\left(\left[\left(\phi^{*} \phi\right)^{n}\right]\right) & =\frac{\left(i e^{2}\right)^{2}}{2 !}\left[\left(2^{2} n(n-1)(n !)^{2}+2^{2} n^{2}(n !)^{2}\right] \mathcal{I}_{14}\right. \\
& +\frac{(-i e)^{2}}{2 !} \frac{i e^{2}}{1 !} 2^{2} n^{2}(n !)^{2} \mathcal{I}_{21}+(n !)^{2}\left(1+A_{\left[\left(\phi^{*} \phi\right)^{2} n\right]}\right)+\text { parte finita },
\end{aligned}
$$

substituindo acima os valores para $\mathcal{I}_{14}$ e $\mathcal{I}_{21}$, temos que

$$
\left.\Gamma^{(2 n)}\left(\left[\left(\phi^{*} \phi\right)^{n}\right]\right)=\frac{(n !)^{2} n(4 n-1)}{4 \pi^{2}} \alpha^{2} \frac{1}{\epsilon}+(n !)^{2} A_{\left[\left(\phi^{*} \phi\right)^{2} n\right]}\right)+ \text { parte finita }
$$

e o contratermo que absorve a divergência em $\Gamma^{(2 n)}\left(\left[\left(\phi^{*} \phi\right)^{n}\right]\right)$ será

$$
A_{\left[\left(\phi^{*} \phi\right)^{\mathrm{n}}\right]}=-\frac{n(4 n-1)}{16 \pi^{2}} \alpha^{2} \frac{1}{\epsilon} .
$$

Podemos resumir os resultados até aqui obtidos na tabela (3.1). 


\begin{tabular}{||c|c|c|c||}
\hline Operadores & Dimensão & categoria & $Z_{\left[\left(\phi^{*} \phi\right)^{n}\right]}=1+A_{\left[\left(\phi^{*} \phi\right)^{n}\right]}$ \\
\hline \hline$\left[\left(\phi^{*} \phi\right)\right]$ & 1 & super-renormalizável & $1-\frac{3}{16 \pi^{2}} \alpha^{2} \frac{1}{\epsilon}$ \\
\hline$\left[\left(\phi^{*} \phi\right)^{2}\right]$ & 2 & super-renormalizável & $1-\frac{7}{8 \pi^{2}} \alpha^{2} \frac{1}{\epsilon}$ \\
\hline$\left[\left(\phi^{*} \phi\right)^{3}\right]$ & 3 & renormalizável & $1-\frac{33}{16 \pi^{2}} \alpha^{2} \frac{1}{\epsilon}$ \\
\hline$\left[\left(\phi^{*} \phi\right)^{4}\right]$ & 4 & não -renormalizável & $1-\frac{15}{4 \pi^{2}} \alpha^{2 \frac{1}{\epsilon}}$ \\
\hline$\left[\left(\phi^{*} \phi\right)^{n}\right]$ & $\mathrm{n}$ & - & $1-\frac{n(4 n-1)}{16 \pi^{2}} \alpha^{2} \frac{1}{\epsilon}$ \\
\hline
\end{tabular}

Tabela 3.1 - Valores da constante de renormalização para os operadores compostos $\left[\left(\phi^{*} \phi\right)^{n}\right]$

\subsection{Equação do Grupo de Renormalização para o Operador Composto}

No capítulo 1, deduzimos a equação do grupo de renormalização para o campo base $\phi$, obtivemos as chamadas funções do grupo de renormalização e discutimos suas propriedades.

Para obtermos a equação do grupo de renormalização para o operador composto e, por conseguinte, a expressão para a dimensão anômala deste operador, devemos proceder de forma análoga àquela que desenvolvemos para o campo base $\phi$.

A função de vértice renormalizada para o operador composto pode ser escrita em termos da não renormalizada (ou vice-versa) por meio de uma renormalização multiplicativa da forma[33][49]

$$
\Gamma_{0, \hat{\mathcal{O}}}^{(m, l)}\left(p_{i}, g_{0} . a_{0}, \epsilon\right)=Z_{\hat{\mathcal{O}}}^{l} Z_{\phi}^{-\frac{m}{2}} \Gamma_{\hat{\mathcal{O}}}^{(m, l)}\left(p_{i}, g . \alpha . \epsilon\right)
$$

sendo que $Z_{\phi}^{-\frac{m}{2}}$ é o fator de renormalização para cada um dos "m"campos $\phi$ e $Z_{\dot{\mathcal{O}}}^{l}$ é o fator de normalização para cada um dos " $l$ "operadores compostos (aqui teremos, para cada função de vértice, apenas um operador composto, tomaremos então $l=1$ ). 
Aplicando o operador $\mu \frac{\partial}{\partial \mu}$ sobre a equação (3.68), levando em conta que $\Gamma_{0}^{(m, l)}$ não depende de $\mu$, podemos escrever diretamente que

$$
\left[\mu \frac{\partial}{\partial \mu}+\gamma_{\hat{\mathcal{O}}}-m \gamma_{\phi}\right] \Gamma_{\hat{\mathcal{O}}}^{(m, 1)}=0
$$

com

$$
\gamma_{\phi}=\frac{\mu}{2} \frac{\partial}{\partial \mu}\left(\log Z_{\phi}\right)=\frac{1}{2} \frac{\mu}{Z_{\phi}} \frac{d Z_{\phi}}{d \mu}
$$

$\mathrm{e}$

$$
\gamma_{\hat{\mathcal{O}}}=\mu \frac{\partial}{\partial \mu}\left(\log Z_{\hat{\mathcal{O}}}\right)=\frac{\mu}{Z_{\hat{\mathcal{O}}}} \frac{d Z_{\hat{\mathcal{O}}}}{d \mu}
$$

sendo que (3.69) é a equação do grupo de renormalização para o operador composto, e $\gamma_{\phi}$ e $\gamma_{\hat{o}}$ são, respectivamente, a dimensão anômala para o campo $\phi$ e para o operador composto $\mathcal{O}$.

Assim como fizemos para $Z_{\phi}$ no capítulo1, aqui vamos escrever $Z_{\hat{\mathcal{O}}}$ como uma expansão em série de Laurent em torno de $\epsilon=0$, ou seja,

$$
Z_{\hat{\mathcal{O}}}=1+\sum_{1}^{\infty} \frac{Z_{\dot{\mathcal{O}}, n}(\alpha)}{\epsilon^{n}}
$$

Aplicando agora o operador $\mu \frac{\partial}{\partial \mu}$ sobre (3.72) temos que

$$
\mu \frac{\partial}{\partial \mu} Z_{\dot{\mathcal{O}}}(\alpha)=\sum_{n=1}^{\infty} \frac{1}{\epsilon^{n}}\left[\mu \frac{\partial \alpha}{\partial \mu} \frac{\partial Z_{\hat{\mathcal{O}}, n}}{\partial \alpha}\right] .
$$

Comparando a equação acima com (3.71) e usando a equação (1.76), temos que

$$
Z_{\hat{\mathcal{O}}} \gamma_{\hat{\mathcal{O}}}=-\alpha \sum_{n=1}^{\infty} \frac{1}{\epsilon^{n-1}} \frac{\partial Z_{\hat{\mathcal{O}}, n}(\alpha)}{\partial \alpha}
$$

Substituindo $Z_{\hat{\mathcal{O}}}$, equação (3.72), na equação acima temos ainda que

$$
\hat{\imath}_{\hat{\mathcal{O}}}+\alpha \frac{\partial Z_{\hat{\mathcal{O}}, 1}(\alpha)}{\partial \alpha}+\sum_{n=1}^{\infty} \frac{1}{\epsilon}\left[\gamma_{\hat{\mathcal{O}}} Z_{\hat{\mathcal{O}}, n}(\alpha)+\alpha \frac{\partial Z_{\hat{\mathcal{O}}, n+1}(\alpha)}{\partial \alpha}\right]=0
$$

e separando na equação acima o termo de ordem $\epsilon^{0}$, podemos escrever que

$$
\gamma_{\hat{\mathcal{O}}}=-\alpha \frac{\partial Z_{\hat{\mathcal{O}, 1}}(\alpha)}{\partial \alpha}
$$


Portanto a dimensão anômala para o operador composto é escrita em termos do produto $Z_{\hat{\mathcal{O}}}=Z_{\left[\left(\phi^{*} \phi\right)^{n}\right]}^{-1} Z_{\phi}^{n}$ de ordem $\frac{1}{\epsilon}$.

Pela tabela (3.1) vemos que,

$$
Z_{\left[\left(\phi^{*} \phi\right)^{n}\right]}=1-\frac{n(4 n-1)}{16 \pi^{2}} \alpha^{2} \frac{1}{\epsilon}
$$

e, de (1.85) e (2.112), temos que

$$
Z_{\phi}=1+\frac{7}{48} \frac{\alpha^{2}}{\pi^{2}} \frac{1}{\epsilon}
$$

sendo que na expressão acima escrevemos apenas a dependência do acolamento $\alpha$ para $Z_{\phi}$. Usando então os resultados (3.77) e (3.78) temos que

$$
Z_{\left[\left(\phi^{*} \phi\right)^{n}\right], 1}=1+\left(\frac{n(4 n-1)}{16}+\frac{7 n}{48}\right) \frac{\alpha^{2}}{\pi^{2}} \frac{1}{\epsilon}
$$

que, ao ser substituida em (3.76), obtemos a dimensão anômala para o operador $\left[\left(\phi^{*} \phi\right)^{n}\right]$, dada por

$$
\gamma_{\left[\left(\phi^{*} \phi\right)^{n}\right]}=-2 n\left(\frac{4 n-1}{16}+\frac{7}{48}\right) \frac{\alpha^{2}}{\pi^{2}}
$$

ou seja, com base em (3.80) podemos garantir que a dimensão anômala dos operadores compostos mantém o mesmo sinal independentemente do valor de $n \geq 1$.

Fazendo a análise dimensional para a equação do grupo de renormalização para o operador composto de forma análoga àquela que fizemos na seção (3.3), é possível ver que a "dimensão efetiva" dos operadores compostos, após a sua renormalização, é dada por

$$
D_{\hat{\mathcal{O}}}=d_{\hat{\mathcal{O}}}+\gamma_{\hat{\mathcal{O}}}
$$

ou ainda, usando a equação (3.80), temos

$$
D_{\left.\left[\left(\phi^{*} \phi\right)^{n}\right]\right)}=d_{\left.\left[\left(\phi^{-} \phi\right)^{n}\right]\right)}-2 n\left(\frac{4 n-1}{16}+\frac{7}{48}\right) \frac{\alpha^{2}}{\pi^{2}}
$$

ou seja, não importando se o operador é super-renormalizável. normalizável ou não renormalizável, o acoplamento de Chern-Simons atua sempre no sentido de diminuir a dimensão canônica do operador, melhorando sua renormalizabilidade. Por exemplo, se tomarmos $n=3$ na equação anterior teremos que

$$
D_{\left.\left[\left(\phi^{-} \phi\right)^{n}\right]\right)}=3-0,23 \alpha^{2},
$$

e o acoplamento $\alpha^{2}$ atua de forma a diminuir a dimensão $D_{\left.\left[\left(\phi^{*} \phi\right)^{n}\right]\right)}$. Porém, apesar de melhorar a renormalizabilidade, está influência não é forte o suficiente para transformar um operador não -renormalizável, $\left[\left(\phi^{*} \delta\right)^{4}\right]$ por exemplo, em renormalizável. Para que isto ocorresse seria necessário um valor $\alpha$ maior que 1 . 


\section{Conclusão}

Nesta tese foi abordado o modelo escalar com uma interação do tipo $\left(\phi^{*} \phi\right)^{3}$, acoplado minimamente a um campo de Chern-Simons. Foram analisadas as funções de grupo de renormalização para o campo básico $\phi$ e a dimensão anômala para o operador composto $\left[\left(\phi^{*} \phi\right)^{n}\right]$. Em ambos os casos a análise foi feita visando discutir a influência do acoplamento de Chern-Simons sobre estas funções.

Para a função $\beta_{g}(g, \alpha)$ do campo $\phi$ constatou-se a presença de dois pontos fixos para $\alpha \neq 0$. O primeiro é o ponto fixo trivial, ou gaussiano, em $g=0$ (este ponto fixo é unicamente determinado, ou seja, independe do valor do acoplamento de Chern-Simons, $\alpha \neq 0$ ). Este ponto fixo gaussiano é estável no ultravioleta, indicando a possibilidade de existência de liberdade assintótica. O segundo ponto fixo, não trivial, é estável no infravermelho. Em função de constante $\alpha$ do acoplamento de Chern-Simons ele é dado por

$$
g_{+}\left(2 b g_{+}+a\right)=c \alpha^{2} .
$$

Para $\alpha \rightarrow 0$ o ponto fixo não trivial infravermelho estável colapsa a $g_{+} \rightarrow 0$, e o ponto fixo ultravioleta estável desaparece em acordo com o resultado conhecido para a teoria $\left(\phi^{*} \phi\right)^{3}$ pura.

Para a dimensão anômala do campo $\phi$ constatou-se que o acoplamento $\alpha$ atua de maneira a diminuir a dimensão canônica do campo $\phi$, indicando uma melhora do comportamento ultravioleta da teoria na presença do termo de Chern-Simons.

A dimensão anômala para o operador composto $\left[\left(\phi^{*} \phi\right)^{n}\right]$ comporta-se de maneira similar a dimensão anômala do campo básico. Ou seja, também atua no sentido de diminuir a dimensão canônica dos operadores compostos, não importando se estes operadores são super-renormalizáveis, renormalizáveis ou não renormalizáveis. 


\section{Apêndice A}

\section{Diagramas de Feynman}

\section{A.1 Função de Dois Pontos}

Aqui vamos listar todos os diagramas que contribuem para a função de Green conexa de dois pontos, segundo cada ordem nas constantes de acoplamento.

- Ordem $\alpha$
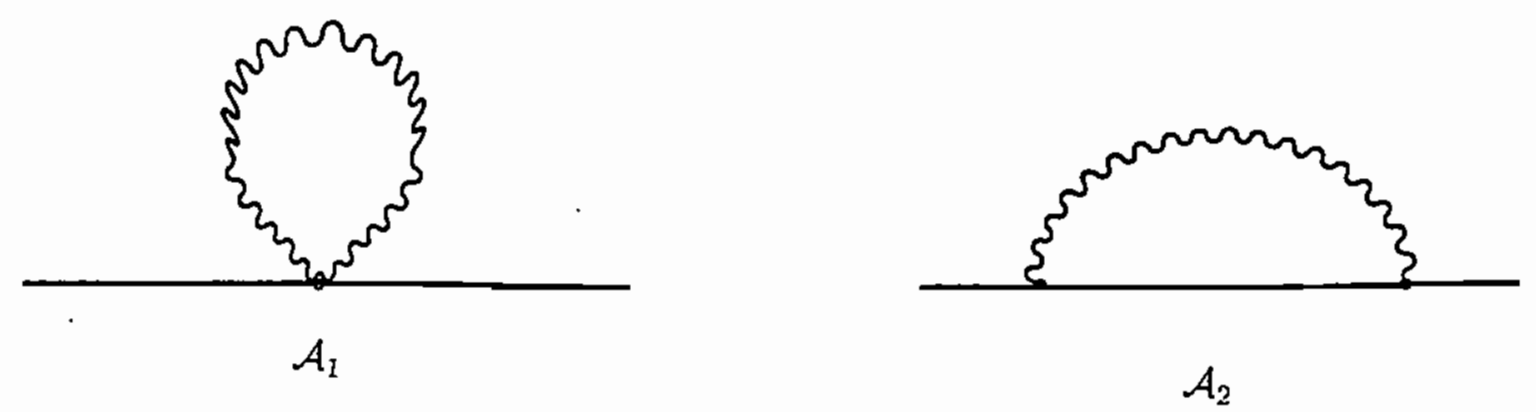

- Ordem $g$

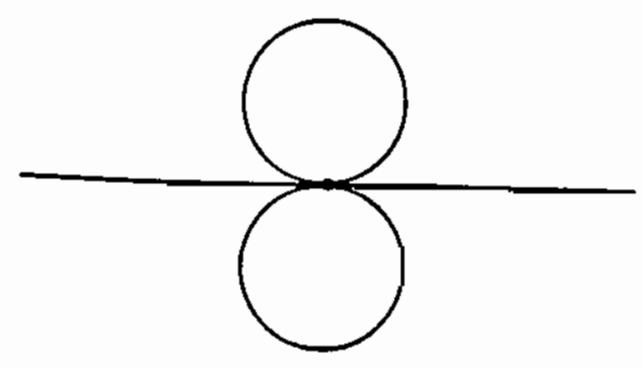

$\mathcal{B}_{1}$ 
- Ordem $\alpha^{2}$

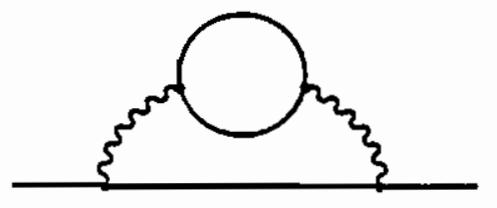

$\mathcal{C}_{1}$

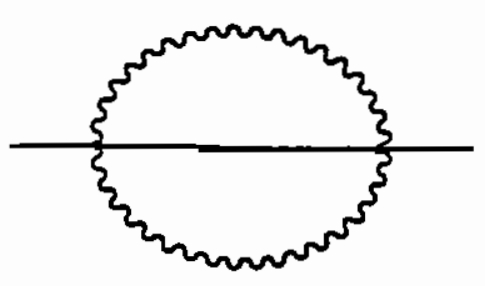

$\mathcal{C}_{2}$

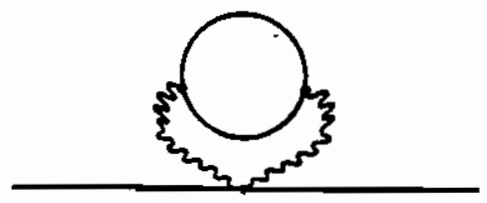

$\mathcal{C}_{6}$
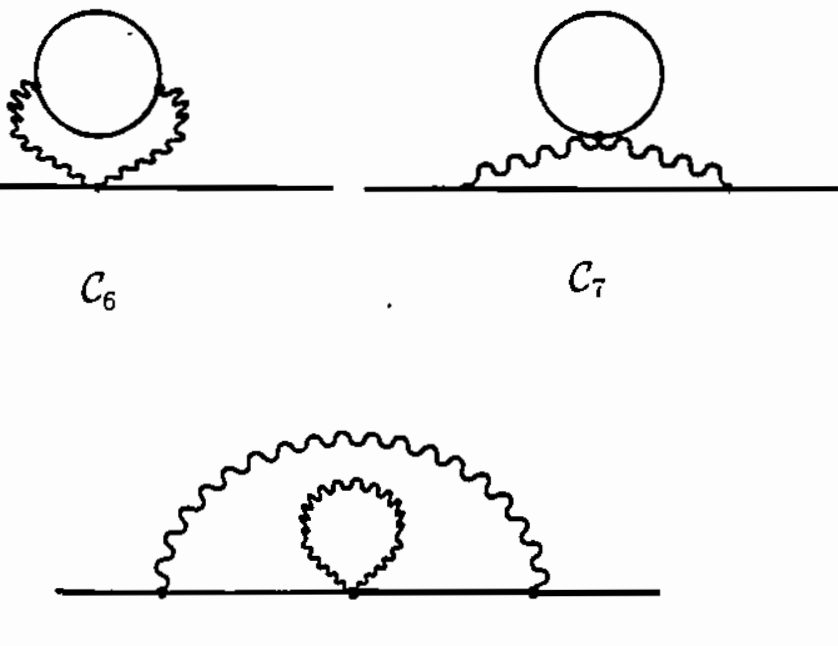

$\mathcal{C}_{10}$

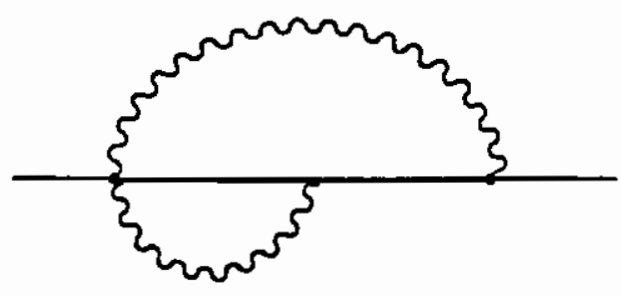

$\mathcal{C}_{9}$
$\mathcal{C}_{i}$

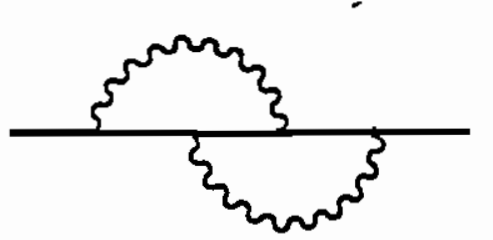

$\mathcal{C}_{3}$

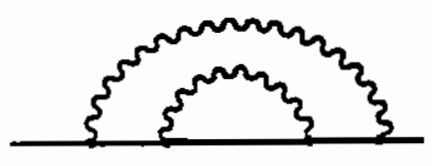

$\mathcal{C}_{4}$

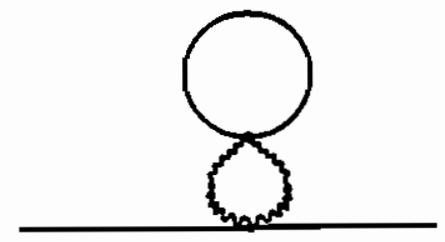

$\mathcal{C}_{8}$

- Ordem $g^{2}$

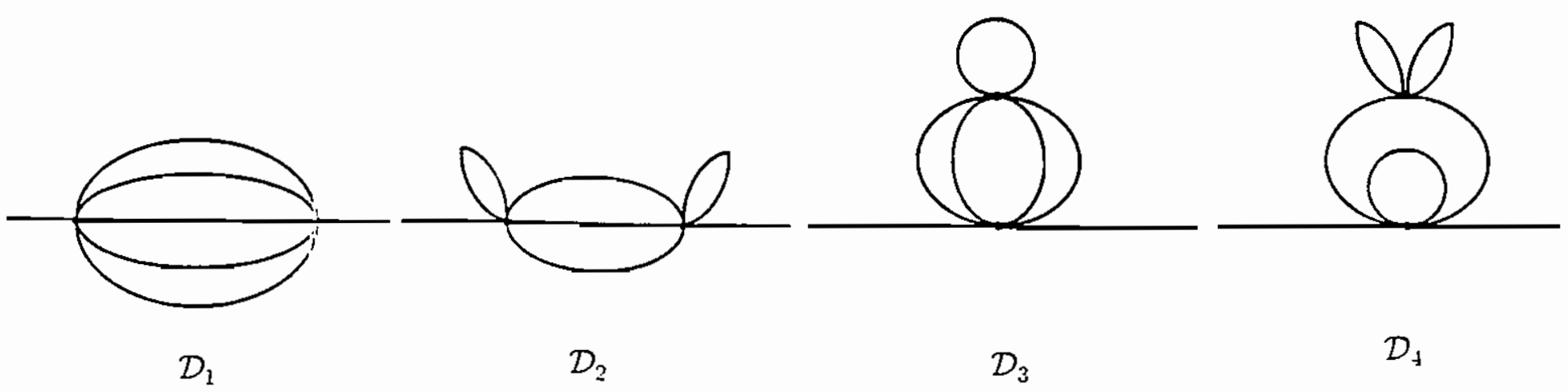


- Ordem $\alpha g$
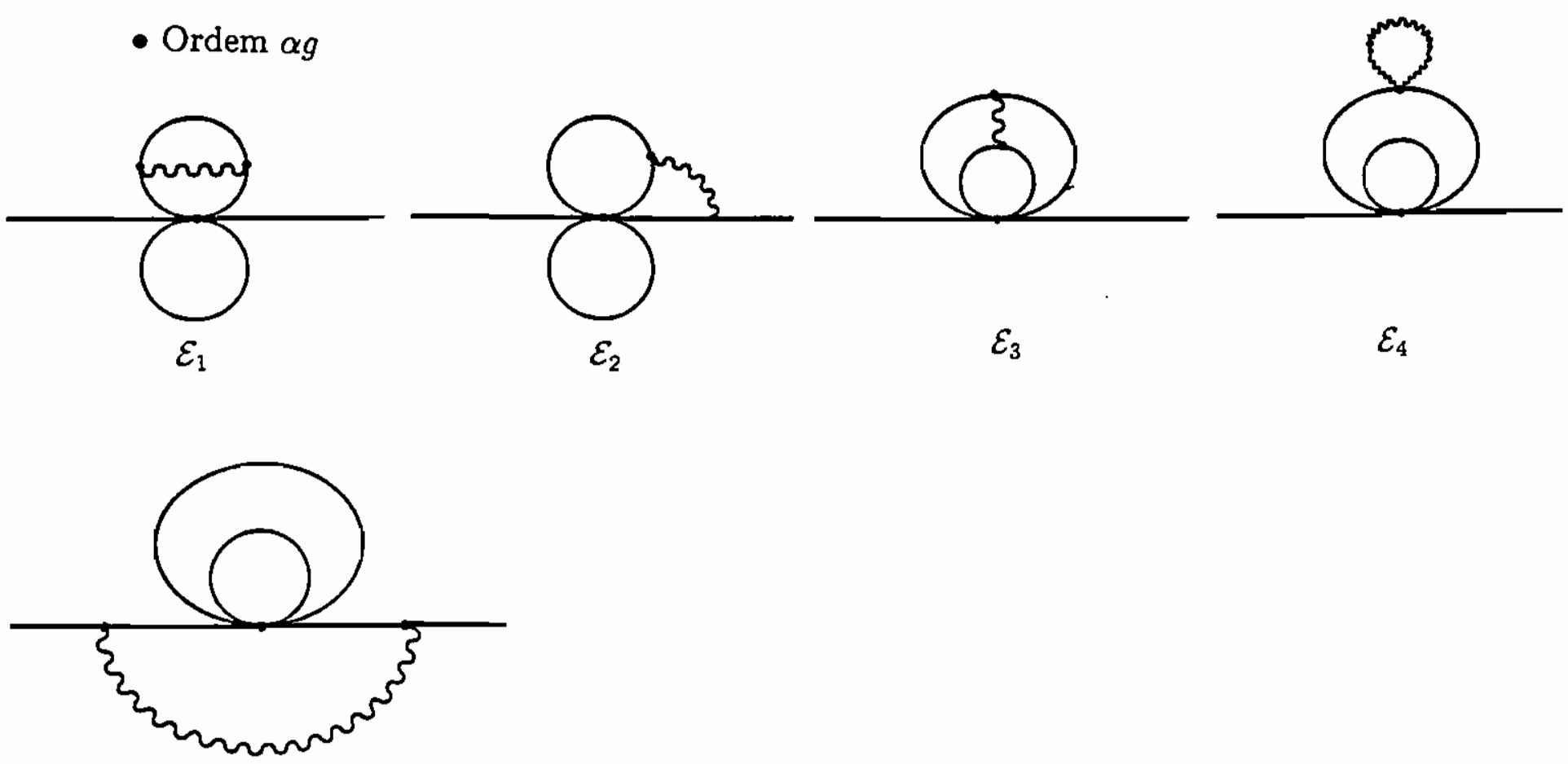

$\mathcal{E}_{5}$

\section{A.2 Função de Seis Pontos}

Aqui vamos listar todos os diagramas que contribuem para a função de Green conexa de seis pontos. segundo cada ordem nas constantes de acoplamento.

- Ordem $g$

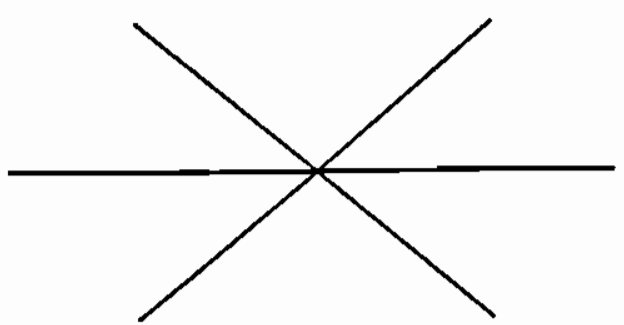


- Ordem $g^{2}$

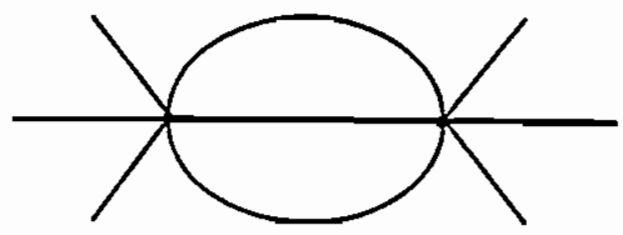

$\mathcal{G}_{1}$

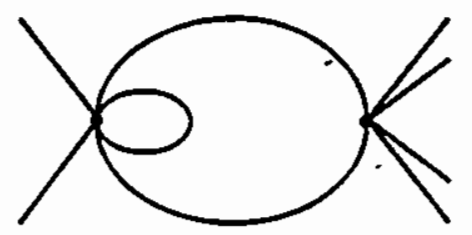

$\mathcal{G}_{2}$

- Ordem ag

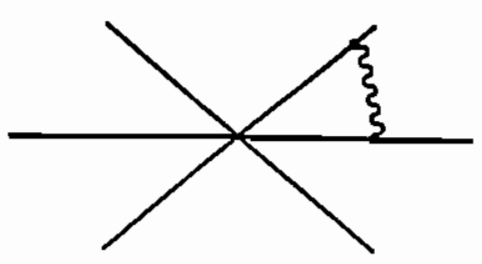

$\mathcal{H}_{1}$

- Ordem $g a^{2}$

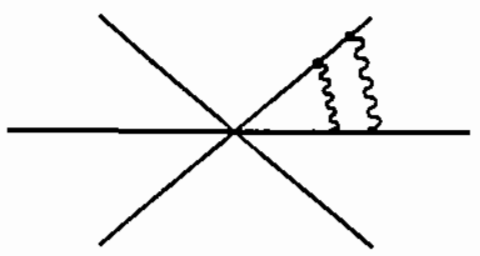

$\mathcal{I}_{1}$

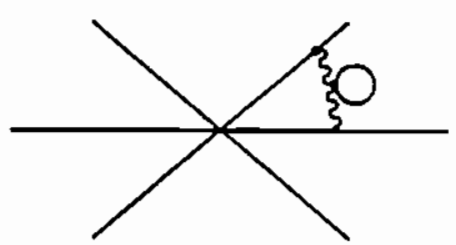

$\mathcal{I}_{5}$

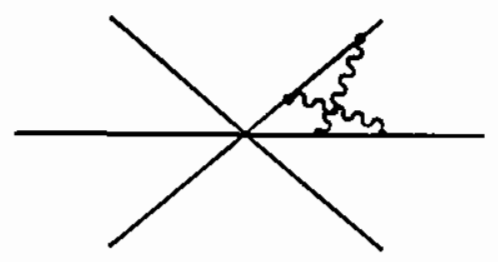

$\mathcal{I}_{2}$

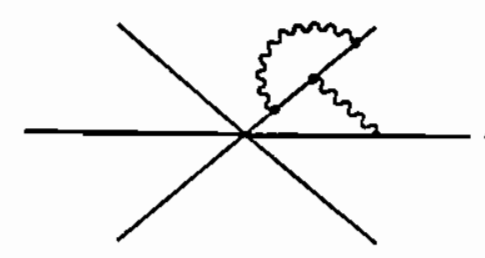

$\mathcal{I}_{6}$

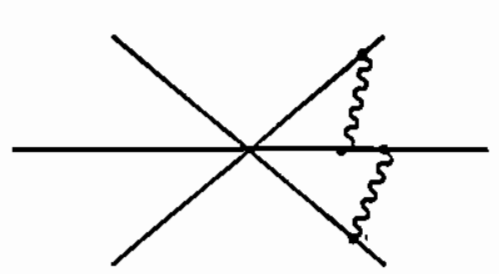

$\mathcal{I}_{3}$

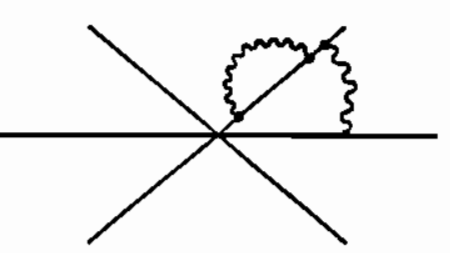

$\mathcal{I}_{\bar{i}}$

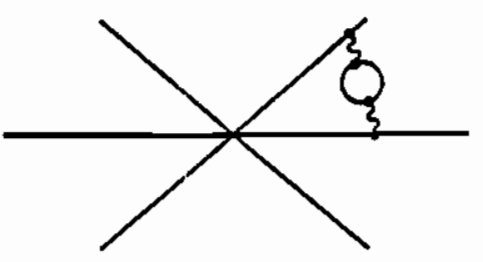

$\mathcal{I}_{4}$

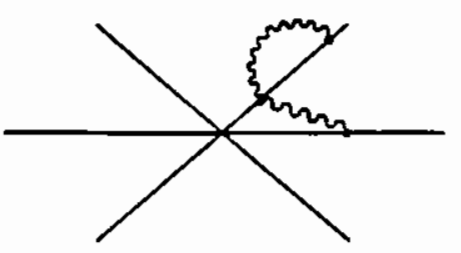

$\mathcal{I}_{8}$ 


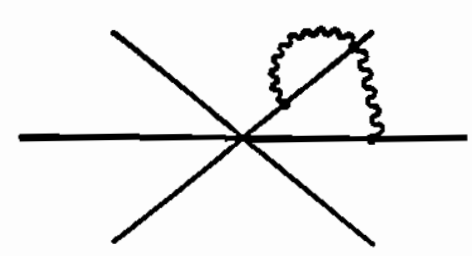

$\mathcal{I}_{9}$

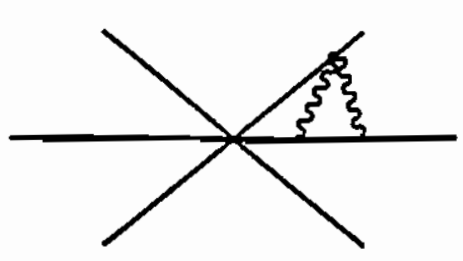

$\mathcal{I}_{13}$

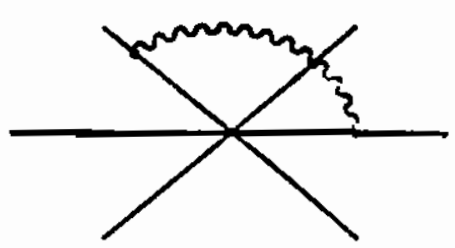

$\mathcal{I}_{10}$

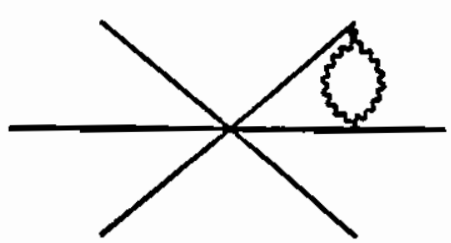

$I_{14}$

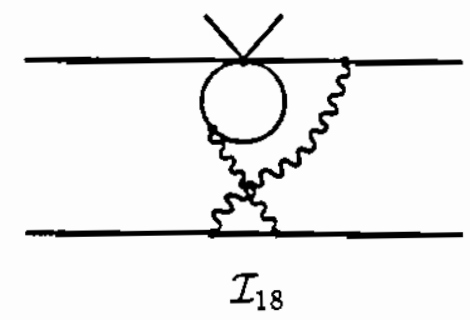

$\mathcal{I}_{17}$
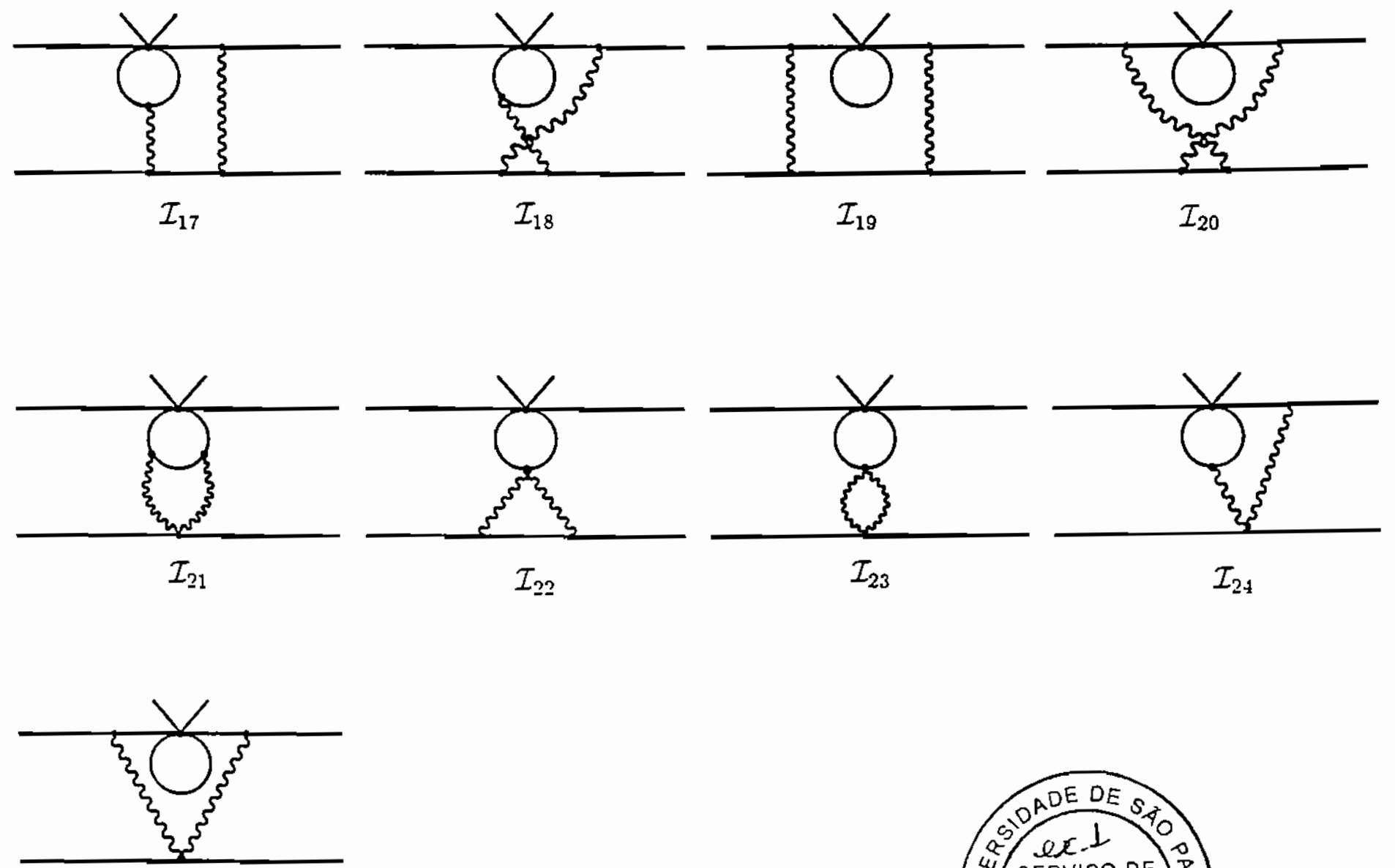

$\mathcal{I}_{25}$
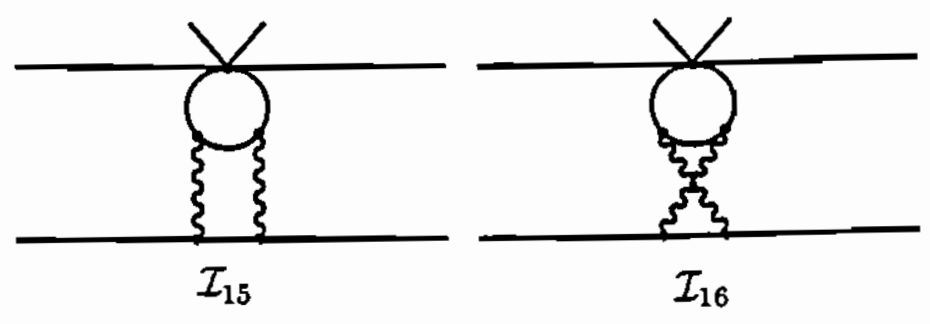

$\mathcal{I}_{12}$ 
- Ordem $\alpha g^{2}$

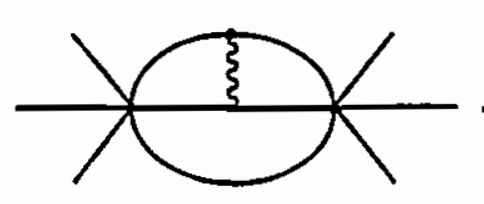

$\mathcal{J}_{1}$

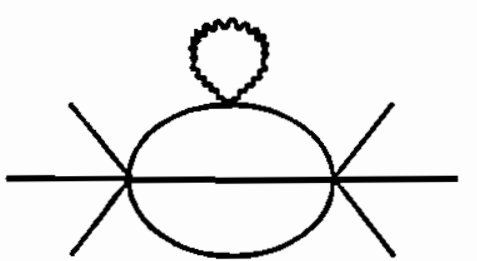

$\mathcal{J}_{5}$

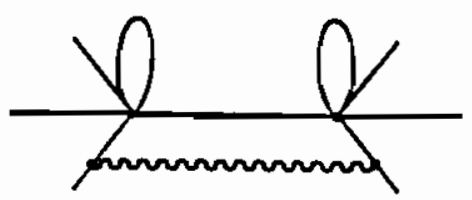

$\mathcal{J}_{9}$

- Ordem $\alpha^{3}$
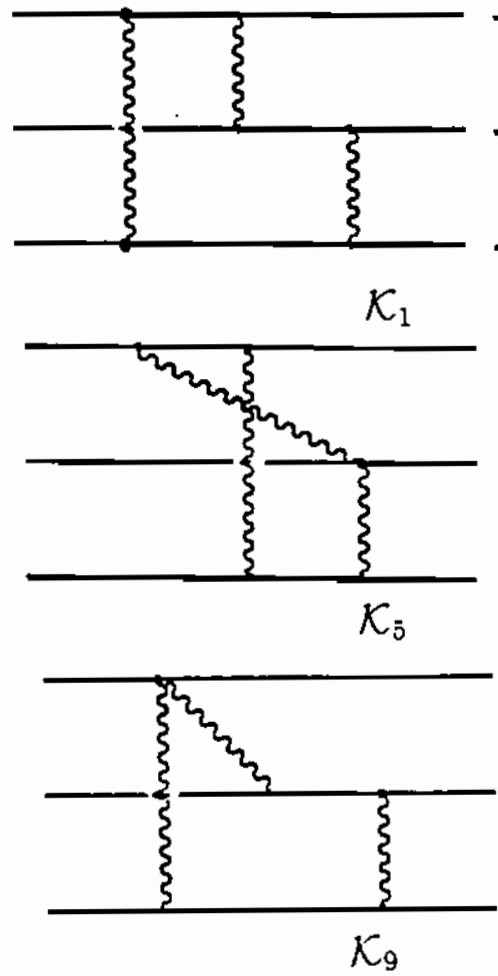

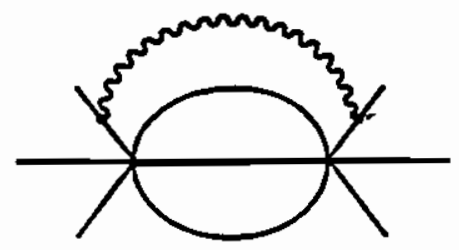

$\mathcal{J}_{2}$

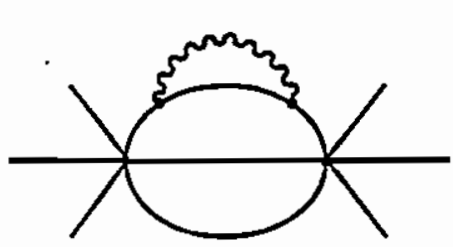

$\mathcal{J}_{6}$
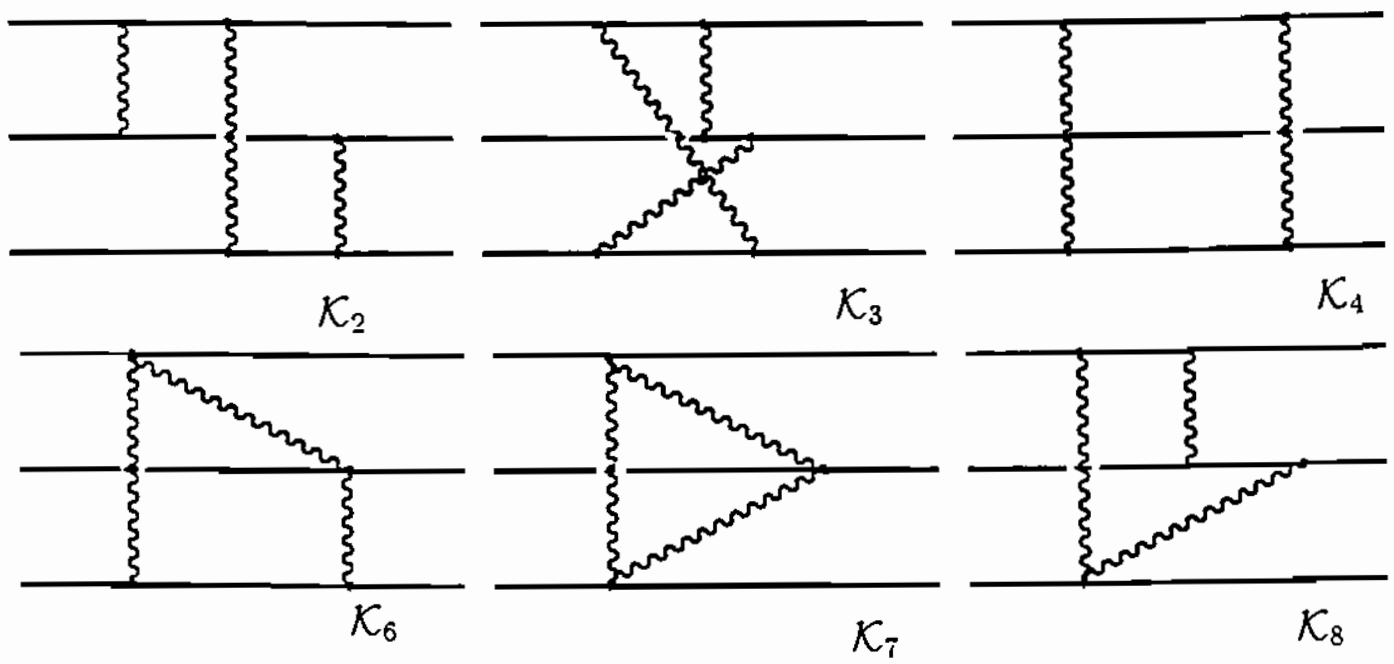
- Ordem $g^{3}$

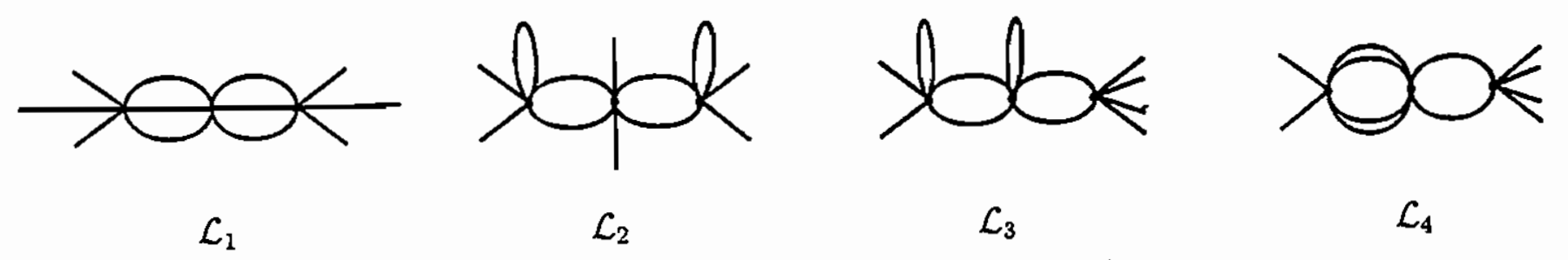

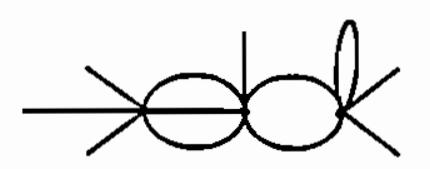

$\mathcal{L}_{5}$

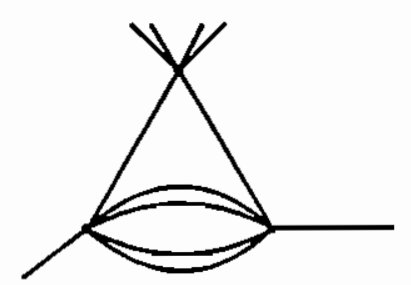

$\mathcal{L}_{9}$

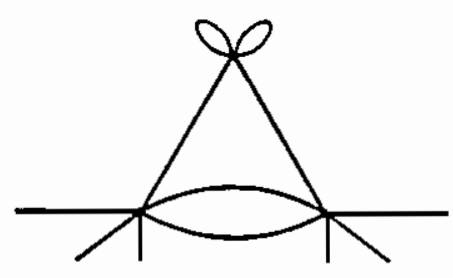

$\mathcal{L}_{13}$

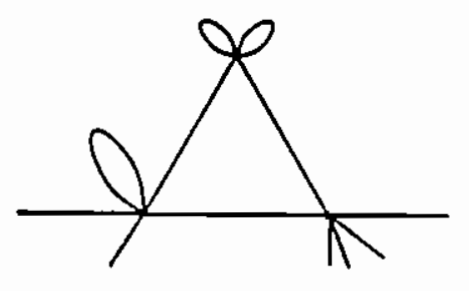

$\mathcal{L}_{17}$

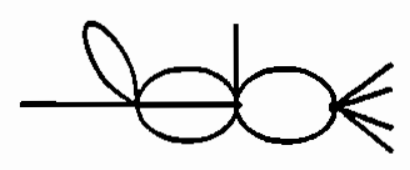

$\mathcal{L}_{6}$

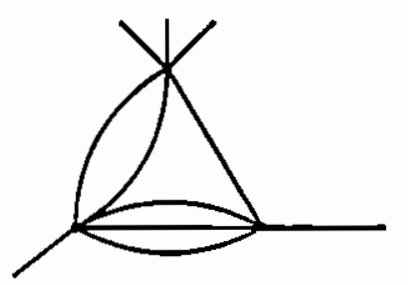

$\mathcal{L}_{10}$

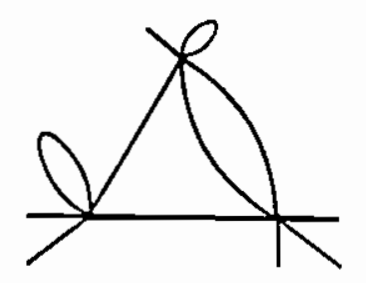

$\mathcal{L}_{14}$
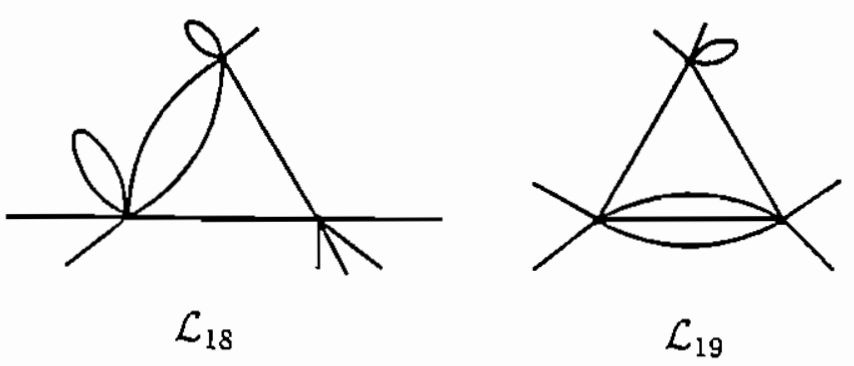

102

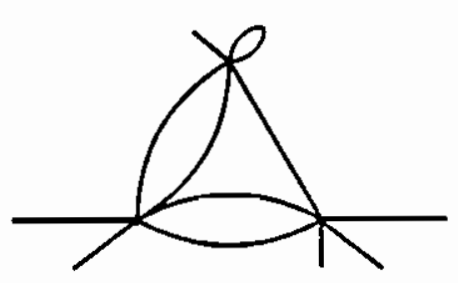

$\mathcal{L}_{15}$

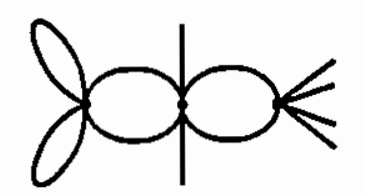

$\mathcal{L}_{7}$

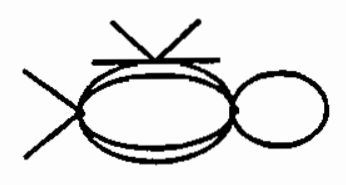

$\mathcal{L}_{8}$

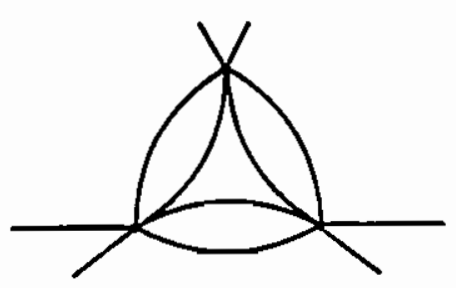

$\mathcal{L}_{11}$

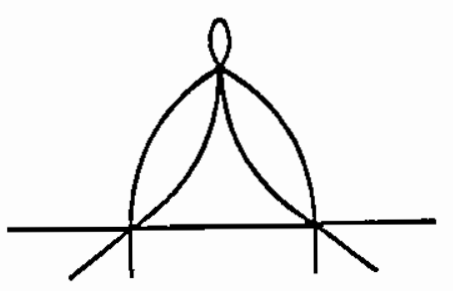

$\mathcal{L}_{12}$

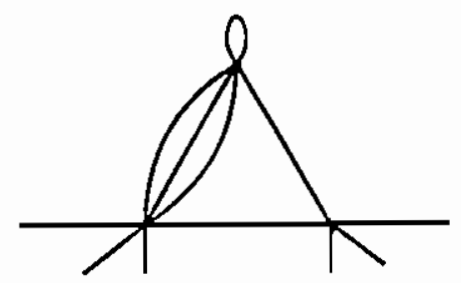

$\mathcal{L}_{16}$ 


\section{Apêndice B}

\section{Tabela}

Neste capítulo, apresentaremos algumas expressões úteis, usadas no texto da tese[33]

a) Formas Gerais das Integrais de Feynman

$$
\begin{aligned}
\int d^{d} k \frac{1}{\left(-k^{2}-2 p . k+C\right)^{\alpha}} & =i \pi^{\frac{d}{2}} \frac{\Gamma\left(\alpha-\frac{d}{2}\right)}{\Gamma(\alpha)}\left[\frac{1}{\left(C+p^{2}\right)^{\alpha-\frac{d}{2}}}\right] \\
\int d^{d} k \frac{k^{\mu}}{\left(-k^{2}-2 p . k+C\right)^{\alpha}} & =i \pi^{\frac{d}{2}} \frac{\Gamma\left(\alpha-\frac{d}{2}\right)}{\Gamma(\alpha)}\left[\frac{-p^{\mu}}{\left(C+p^{2}\right)^{\alpha-\frac{d}{2}}}\right] \\
\int d^{d} k \frac{k^{\mu} k^{\nu}}{\left(-k^{2}-2 p . k+C\right)^{\alpha}} & =i \pi^{\frac{d}{2}} \frac{1}{\Gamma(\alpha)}\left[\Gamma\left(\alpha-\frac{d}{2}\right) \frac{p^{\mu} p^{\nu}}{\left(C+p^{2}\right)^{\alpha-\frac{d}{2}}}\right. \\
& \left.-\frac{1}{2} \Gamma\left(\alpha-1-\frac{d}{2}\right) \frac{g^{\mu \nu}}{\left(C+p^{2}\right)^{\alpha-\frac{d}{2}-1}}\right] \\
\int d^{d} k \frac{k^{\mu} k^{\nu} k^{\rho} k^{\sigma}}{\left(-k^{2}+C\right)^{\alpha}} & =i \pi^{\frac{d}{2}} \frac{\Gamma\left(\alpha-\frac{d}{2}-2\right)}{\Gamma(\alpha)} C^{\frac{d}{2}+2-\alpha} \frac{1}{4}\left(g^{\mu \nu} g^{\rho \sigma}+g^{\mu \rho} g^{\nu \sigma}+g^{\mu \sigma} g^{\rho \nu}\right) \\
\int d^{d} k \frac{k^{\mu_{1}} k^{\mu_{2}} \ldots k^{\mu_{P}}}{\left(-k^{2}+C\right)^{\alpha}} & =(-1)^{f r a c 3 P 2} i \pi^{\frac{d}{2}} \frac{\Gamma\left(\alpha-\frac{P}{2}-\frac{d}{2}\right)}{\Gamma(\alpha)} C^{\frac{d}{2}+\frac{P}{2}-\alpha} 2^{-\frac{P}{2}} \sum_{p e r m .} g^{\mu_{1} \mu_{2}} g^{\mu_{1} \mu_{2}} g^{\mu_{1} \mu_{2}}
\end{aligned}
$$

b) Parametrizações de Feynman

$$
\begin{aligned}
\frac{1}{A^{\alpha} B^{\beta}} & =\frac{\Gamma(\alpha+\beta)}{\Gamma(a) \Gamma(\beta)} \int_{0}^{1} d x \frac{x^{\alpha-1}(1-x)^{\beta-1}}{[A x+B(1-x)]^{\alpha+\beta}} \\
\frac{1}{A B C} & =2 \int_{0}^{1} d y y \int_{0}^{1} d x \frac{1}{[C(1-y)+y(A x+(1-x) B)]^{3}} \\
& =\int_{0}^{1} d y \int_{0}^{1} d x \frac{1}{[A+(B-A) y+(C-B) x]^{3}}
\end{aligned}
$$




$$
\begin{aligned}
\frac{1}{A^{\alpha} B^{\beta} \ldots E^{\epsilon}} & =\frac{\Gamma(\alpha+\beta+\ldots+\epsilon)}{\Gamma(\alpha) \Gamma(\beta) \ldots \Gamma(\epsilon)} \int_{0}^{1} d x d y \ldots d z \delta(1-x-y-\ldots-z) \\
& \times \frac{x^{\alpha-1} y^{\beta-1} \ldots z^{\epsilon-1}}{(A x+B y+\ldots E)^{\alpha+\beta+\ldots+\epsilon}}
\end{aligned}
$$

\section{c) Fórmulas Gerais}

$$
\begin{gathered}
\int_{0}^{1} d x x^{\nu-1}(1-x)^{\mu-1}=\frac{\Gamma(\mu) \Gamma(\nu)}{\Gamma(\mu+\nu)} \\
\Gamma(-n+\epsilon)=\frac{(-1)^{n}}{n !}\left[\frac{1}{\epsilon}+\psi_{n+1}+\epsilon\left(\frac{\pi^{2}}{6}+\frac{\psi_{n+1}^{2}}{2}-\frac{\psi_{n+1}^{\prime}}{2}+\ldots\right)\right]
\end{gathered}
$$

sendo $\gamma=-\psi_{1}(1)=0.577$ (constante de Euler-Mascheroni) e $\psi_{1}=\gamma$ e $\psi_{n+1}^{\prime}=1+\frac{1}{2}+$ $\ldots+\frac{1}{n}-\gamma, n \geq 1$.

$$
n \Gamma(n)=\Gamma(n+1)
$$

$\operatorname{com} \Gamma(1)=\Gamma(2)=1$ e $\Gamma(1 / 2)=\sqrt{\pi}$.

$$
\Gamma(2 x)=\frac{2^{2 x}}{\sqrt{4 \pi}} \Gamma(x) \Gamma\left(\frac{1}{2}+x\right)
$$

ou ainda,

$$
\Gamma\left(\frac{1}{2}+x\right)=2^{-2 x} \sqrt{4 \pi} \frac{\Gamma(2 x)}{\Gamma(x)} .
$$

Até a ordem $\epsilon$, temos também que

$$
\begin{gathered}
\Gamma\left(\frac{1}{2}+\epsilon\right)=\sqrt{\pi}[1-\epsilon(\gamma+2 \ln 2)], \\
\Gamma\left(\frac{3}{2}+\epsilon\right)=\frac{\sqrt{\pi}}{2}[1+\epsilon(2-\gamma-2 \ln 2)], \\
\varepsilon^{\alpha \beta \rho} \varepsilon_{\alpha \theta \lambda}=g_{\theta}^{\beta} g_{\lambda}^{\rho}-g_{\lambda}^{\beta} g_{\theta}^{\rho}
\end{gathered}
$$




\section{Apêndice C}

\section{Integrais Gerais dos Loops de Momentos}

Com o intuito de facilitar a leitura da tese escrevemos, quando possível, as integrais dos "loops" de momento em três formas, segundo a potência do momento do numerador do integrando, as quais chamamos de

$$
\begin{aligned}
I_{(a)} & =\int d^{d} q \frac{1}{\left[-q^{2}\right]^{\alpha}\left[-(q+p)^{2}\right]^{\beta}} \\
I_{(b)}^{\mu} & =\int d^{d} q \frac{q^{\mu}}{\left[-q^{2}\right]^{\alpha}\left[-(q+p)^{2}\right]^{\beta}} \\
I_{(c)}^{\mu \nu} & =\int d^{d} q \frac{q^{\mu} q^{\nu}}{\left[-q^{2}\right]^{\alpha}\left[-(q+p)^{2}\right]^{\beta}} .
\end{aligned}
$$

Neste apêndice vamos resolver estas integrais, inclusive as eventuais integrais paramétricas, obtendo uma solução geral em termos dos coeficientes $\alpha$ e $\beta$ e, é claro, do momento $p$. Estes resultados são usados diretamente no corpo do trabalho, segundo cada caso específico. Dito isto, vamos começar com a integral $I_{(a)}$,

$$
I_{(a)}=\int d^{d} q \frac{1}{\left[-q^{2}\right]^{\alpha}\left[-(q+p)^{2}\right]^{\beta}} .
$$

Usando na equação acima a parametrização (B.6) do apêndice B considerando $A=-q^{2}$ e $B=-(q+p)^{2}$ temos que

$$
I_{(a)}=\frac{\Gamma(\alpha+\beta)}{\Gamma(\alpha) \Gamma(\beta)} \int_{0}^{1} d x x^{\alpha-1}(1-x)^{\beta-1} \int d^{d} q \frac{1}{\left[-q^{2}-2 q \cdot p(1-x)+p^{2}(x-1)\right]^{\alpha+\beta}} .
$$

Calculando a integral em $q$, usando (B.1), temos 


$$
I_{(a)}=i \pi^{\frac{d}{2}} \frac{\Gamma(\alpha+\beta-d / 2)}{\Gamma(\alpha) \Gamma(\beta)} \int_{0}^{1} d x x^{\alpha-1}(1-x)^{\beta-1}\left[p^{2}(x-1)+p^{2}(1-x)^{2}\right]^{\frac{d}{2}-\alpha-\beta} .
$$

a qual, escrita convenientemente, toma a forma

$$
I_{(a)}=i \pi^{\frac{d}{2}} \frac{\Gamma(\alpha+\beta-d / 2)}{\Gamma(\alpha) \Gamma(\beta)}\left[p^{2}\right]^{\frac{d}{2}-\alpha-\beta} \int_{0}^{1} d x x^{\frac{d}{2}-\beta-1}(1-x)^{\frac{d}{2}-\alpha-1} .
$$

Usando ainda (B.9), para resolver a integral em $x$ acima, e (B.11), vemos que $I_{(a)}$ pode ser escrita como

$$
I_{(a)}=i \pi^{\frac{d}{2}} \frac{\Gamma\left(\frac{d}{2}-\alpha\right) \Gamma\left(\frac{d}{2}-\beta\right) \Gamma(\alpha+\beta-d / 2)}{\Gamma(\alpha) \Gamma(\beta) \Gamma(d-\alpha-\beta)} .
$$

Vamos calcular a integral $I_{(b)}^{\mu}$ mostrada em (C.2)

$$
I_{(b)}^{\mu}=\int d^{d} q \frac{q^{\mu}}{\left[-q^{2}\right]^{\alpha}\left[-(q+p)^{2}\right]^{\beta}} .
$$

Usando a paramentrização (B.6) com com $A=-q^{2}$ e $B=-(q+p)^{2}$, a equação acima toma a forma

$$
I_{(b)}^{\mu}=\frac{\Gamma(\alpha+\beta)}{\Gamma(\alpha) \Gamma(\beta)} \int_{0}^{1} d x x^{\alpha-1}(1-x)^{\beta-1} \int d^{d} q \frac{q^{\mu}}{\left[-q^{2}-2 q \cdot p(1-x)+p^{2}(x-1)\right]^{\alpha+\beta}} .
$$

Calculando a integral em $q$ usando (B.2)

$$
I_{(b)}^{\mu}=i \pi^{\frac{d}{2}} \frac{\Gamma(\alpha+\beta-d / 2)}{\Gamma(\alpha) \Gamma(\beta)} \int_{0}^{1} d x x^{\alpha-1}(1-x)^{\beta-1} \frac{\left[-p^{\mu}(1-x)\right]}{\left[p^{2}(x-1)+p^{2}(1-x)^{2}\right]^{\alpha+\beta-\frac{d}{2}}},
$$

que após alguma álgebra toma a forma

$$
I_{(b)}^{\mu}=-i \pi^{\frac{d}{2}} \frac{\Gamma(\alpha+\beta-d / 2)}{\Gamma(\alpha) \Gamma(\beta)} p^{\mu}\left[p^{2}\right]^{\frac{d}{2}-\alpha-\beta} \int_{0}^{1} d x x^{\frac{d}{2}-\beta}(1-x)^{\frac{d}{2}-\alpha-1}
$$

e usando ainda a equação (B.9) temos que $I_{\langle b\rangle}^{\mu}$ temos que $I_{\langle b\rangle}^{\mu}$ toma a forma,

$$
I_{(b)}^{\mu}=-i \pi^{\frac{d}{2}} \frac{\Gamma\left(\frac{d}{2}+1-\alpha\right) \Gamma\left(\frac{d}{2}-\beta\right) \Gamma(\alpha+\beta-d / 2)}{\Gamma(\alpha) \Gamma(\beta) \Gamma(d+1-\alpha-\beta)} p^{\mu}\left[-p^{2}\right]^{\frac{d}{2}-\alpha-\beta} .
$$

Por fim, para a integral $I_{(c)}^{\mu \nu}$, dada em (C.3)

$$
I_{(c)}^{\mu \nu}=\int d^{d} q \frac{q^{\mu} q^{\nu}}{\left[-q^{2}\right]^{\alpha}\left[-(q+p)^{2}\right]^{\beta}} .
$$


a qual, ao usarmos novamente a parametrização (B.6), considerando $A=-q^{2}$ e $B=$ $-(q+p)^{2}$, será

$$
I_{(c)}^{\mu \nu}=\frac{\Gamma(\alpha+\beta)}{\Gamma(\alpha) \Gamma(\beta)} \int_{0}^{1} d x x^{\alpha-1}(1-x)^{\beta-1} \int d^{d} q \frac{q^{\mu} q^{\nu}}{\left[-q^{2}-2 q \cdot p(1-x)+p^{2}(x-1)\right]^{\alpha+\beta}} .
$$

Usando agora (B.3), lançando mão da igualdade $(\alpha+\beta-1-d / 2) \Gamma(\alpha+\beta-1-d / 2)=$ $\Gamma(\alpha+\beta-d / 2)$ temos

$$
\begin{aligned}
I_{(c)}^{\mu \nu}= & i \pi^{\frac{d}{2}} \frac{\Gamma(\alpha+\beta-1-d / 2)}{\Gamma(\alpha) \Gamma(\beta)} \int_{0}^{1} d x x^{\alpha-1}(1-x)^{\beta-1} \\
& {\left[\left(\alpha+\beta-1-\frac{d}{2}\right) \frac{\left[p^{\mu} p^{\nu}(1-x)^{2}\right]}{\left[p^{2}(x-1)+p^{2}(1-x)^{2}\right]^{\alpha+\beta-\frac{d}{2}}}\right.} \\
- & \left.\frac{g^{\mu \nu}}{\left[p^{2}(x-1)+p^{2}(1-x)^{2}\right]^{\alpha+\beta-1-\frac{d}{2}}}\right] .
\end{aligned}
$$

que após alguma álgebra, explicitamendo as integrais paramétricas, será dada por

$$
\begin{aligned}
I_{(c)=}^{\mu \nu}= & i \pi^{\frac{d}{2}} \frac{\Gamma(\alpha+\beta-1-d / 2)}{2 \Gamma(\alpha) \Gamma(\beta)}\left[2\left(\alpha+\beta-1-\frac{d}{2}\right) p^{\mu} p^{\nu}\left[p^{2}\right]^{\frac{d}{2}-\alpha-\beta}\right. \\
& \left.\int_{0}^{1} d x x^{\frac{d}{2}-1-\beta}(1-x)^{\frac{d}{2}+1-\alpha}-g^{\mu \nu}\left[p^{2}\right]^{\frac{d}{2}+1-\alpha-\beta} \int_{0}^{1} d x x^{\frac{d}{2}-\beta}(1-x)^{\frac{d}{2}-\alpha}\right] .
\end{aligned}
$$

Efetuando as integrais em $x$ usando (B.9), bem como (B.11), temos finalmente que

$$
\begin{aligned}
I_{(c)}^{\mu \nu}= & i \pi^{\frac{d}{2}} \frac{\Gamma\left(\frac{d}{2}+1-\alpha\right) \Gamma\left(\frac{d}{2}+1-\beta\right) \Gamma(\alpha+\beta-1-d / 2)}{2 \Gamma(\alpha) \Gamma(\beta) \Gamma(d+2-\alpha-\beta)}\left[-p^{2}\right]^{\frac{d}{2}+1-\alpha-\beta} \\
& {\left[\frac{2\left(\alpha+\beta-1-\frac{d}{2}\right)\left(\frac{d}{2}+1-\alpha\right)}{\left(\frac{d}{2}-\beta\right)} \frac{p^{\mu} p^{\nu}}{\left[-p^{2}\right]}-g^{\mu \nu}\right] }
\end{aligned}
$$

Sendo que (C.4),(C.5) e (C.6) são as expressões que são usadas recorrentemente no texto da tese. 


\section{Apêndice D}

\section{Função de Vértice de Dois Pontos para o Campo $A_{\mu}$}

Colema e Hill[47] provaram que, em três dimensões, para todas as ordens da teoria de perturbação, todas as correções para o campo de Chern-Simons anulam-se identicamente. Sendo que este resultado é válido para teorias em que o campo abeliano de Chern-Simons interage com campos escalares, espinoriais ou vetoriais.

Neste apêndice vamos mostrar explicitamente que a função de vértice de dois pontos para o campo $A_{\mu}$, até a ordem de perturbação que estamos adotando neste trabalho, $\alpha^{2}$, é finita.

A função de vértice de dois pontos para o campo $A_{\mu}$ é dada graficamente pela figura (D.1).

O diagrama (a), usando o resultado mostrado para $\Delta_{1}$, equação (2.45), é nulo. O mesmo ocorre com (c) e (e); como pode ser constatado pelo resultado para $A_{2}(p)$, equação (2.42), e para o diagrama (d); ver resultado para $\Theta_{\mu}(p)$, equação (2.27). O diagrama (b), por sua vez, é finito, ver resultado para $\Pi_{\alpha \beta}(p)$, equação (2.17).

Desta maneira, resta-nos analisar os diagramas (f) e (g), e assim determinar o valor do contratermo (h).

Chamando de (f') o diagrama (f) com as pernas externas amputadas temos, pela figura (D.2), que

$$
\left(f^{\prime}\right)=\int \mathcal{D} k \mathcal{D} p \frac{i}{(p-q)^{2}} \frac{i}{(k+p)^{2}} \varepsilon^{\mu \nu \rho} \frac{k_{\rho}}{k^{2}} .
$$

Fazendo primeiramente a integral em $k$, temos que

$$
I_{k}=\int \mathcal{D} k \varepsilon^{\mu \nu \rho} \frac{k_{\rho}}{k^{2}} \frac{i}{(k+p)^{2}} .
$$





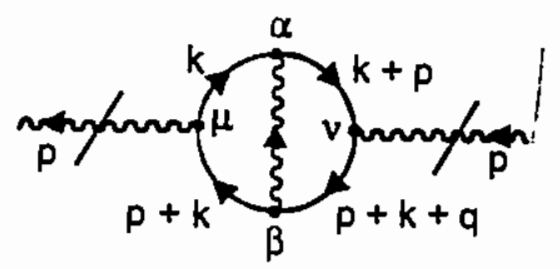

Figura D.3: Diagrama (g).

Usando o apêndice B e fazendo uma parametrização da forma (B.6), podemos escrever $I_{k}$ como

$$
I_{k}=\int_{0}^{1} d x \int \mathcal{D} k \varepsilon^{\mu \nu \rho} \frac{i k_{\rho}}{\left[-k^{2}-x p^{2}-2 x p . k\right]^{2}},
$$

usando ainda (B.2) para resolver a integral em $k$ temos

$$
I_{k}=\frac{1}{2^{2} \pi} \xi^{\mu \nu \rho} \frac{p_{\rho}}{\left(-p^{2}\right)^{2-\frac{d}{2}}} \int_{0}^{1} d x x^{\frac{d}{2}-1}(1-x)^{\frac{d}{2}-2},
$$

ou ainda, usando (B.9),

$$
\int_{0}^{1} d x x^{\frac{d}{2}-1}(1-x)^{\frac{d}{2}-2}=\frac{\Gamma\left(\frac{d}{2}\right)\left(\frac{d}{2}-1\right)}{(d-1)}=\frac{\pi}{2} .
$$

Portanto $I_{k}$ tem como resultado

$$
I_{k}=\frac{1}{16} \varepsilon^{\mu \nu \rho} \frac{p_{r} h o}{\left[-p^{2}\right]^{\frac{1}{2}+\frac{\varepsilon}{2}}}
$$

que ao ser substituído em (D.1) nos leva a

$$
\left(f^{\prime}\right)=-\frac{i}{16} \xi^{\mu \nu \rho} \int \mathcal{D} p \frac{p_{\rho}}{\left[-p^{2}\right]^{\frac{1}{2}+\frac{\frac{c}{2}}{2}}\left[-(p-q)^{2}\right]^{2}} .
$$

Procedendo analogamente ao cálculo de $i_{k}$, ou usando diretamente a forma geral da integral $I_{(b)}^{\mu}$, mostrada no apêndice C, equação (C.2), tem-se diretamente que

$$
\left(f^{\prime}\right)=\frac{1}{96 \pi^{2}} z^{\mu \nu \rho} q_{\rho} \frac{1}{\epsilon}+\text { parte finita. }
$$

Chamando de $\left(\mathrm{g}^{\circ}\right)$ o diagrama $(\mathrm{g})$ com as pernas externas amputadas temos, pela figura (D.3), que 


$$
\begin{aligned}
\left(g^{\prime}\right)(p)= & \int \mathcal{D} k \mathcal{D} q \frac{i}{k^{2}} \frac{i}{(p+k)^{2}} \frac{i}{(k+q)^{2}} \frac{i}{(k+q+p)^{2}} \\
& \left.\times(2 k+q)_{\alpha} 92 p+2 k+q\right)_{\beta}(2 k+p)_{\mu}(2 k+2 q+p)_{\nu} \varepsilon^{\alpha \beta \gamma} \frac{a_{\gamma}}{q^{2}} .
\end{aligned}
$$

Após usarmos em (D.7) as propriedades de anti-simetria do tensor de Levi-Civita, vemos que o diagrama $\left(g^{\prime}\right)$ pode ser escrito como

$$
\left(g^{\prime}\right)=p^{\beta} I_{\beta \mu \nu}(p)
$$

com

$$
I_{\beta \mu \nu}(p)=\int \mathcal{D} k \mathcal{D} q \frac{\left[4 k_{\alpha} p_{\beta}(2 k+p)_{\mu}(2 k+2 q+p)_{\nu}\right]}{\left[k^{2}\right]\left[(p+k)^{2}\right]\left[(q+k)^{2}\right]\left[(p+q+k)^{2}\right]} \varepsilon^{\alpha \beta \lambda} \frac{q_{\lambda}}{q^{2}},
$$

que por sua vez é logaritimicamente divergênte. Portanto, para obtermos a parte divergênte de $I_{\beta \mu \nu}(p)$, basta-nos tomar o primeiro termo na expansão de Taylor em torno de $p_{i}=0$, ou seja, calcular $I_{\beta \mu \nu}(p=0)$, que só pode ser da forma

$$
\begin{aligned}
I_{\beta \mu \nu}(p=0) & =\varepsilon_{\beta \mu \nu} C \text { (independente dep) } \\
& =16 \int \mathcal{D} k \mathcal{D} q \frac{\varepsilon^{\alpha \beta \gamma} k_{\mu}(k+q)_{\nu} k_{\alpha} q_{\gamma}}{q^{2}\left[k^{2}\right]^{2}\left[(k+q)^{2}\right]^{2}} .
\end{aligned}
$$

De (D.10), temos ainda que

$$
6 C=\varepsilon^{\beta \mu \nu} \varepsilon_{\beta \mu \nu}=-16 \int \mathcal{D} q \frac{q^{2} g_{\mu \nu}-q_{\mu} q_{\nu}}{q^{2}} I_{\mu \nu}(q)
$$

com

$$
I_{\mu \nu}(q)=\int \mathcal{D} k \frac{k_{\mu} k_{\nu}}{\left[k^{2}\right]\left[(k+q)^{2}\right]^{2}} .
$$

A integral acima pode ser calculada usando o apêndice C, equação $I_{(c)}^{\mu \nu}$, cuja solução é vista em (C.6). Usando este resultado podemos escrever que

$$
I_{\mu \nu}(q)=-\frac{i}{32} \frac{g_{\mu \nu}}{\left(-q^{2}\right)^{\frac{3}{2}+\frac{1}{2}}}
$$

que, ao ser substituída em (D.11), nos leva a

$$
6 C=-\frac{i}{2} \int \mathcal{D} q \frac{1}{\left[-q^{2}+i \eta\right]^{\frac{3}{2}+\frac{\epsilon}{2}}}
$$

ou ainda, usando (B.1), a integral acima tem como solução

$$
I_{q}=\frac{i}{(2 \pi)^{d}} \pi^{\frac{d}{2}} \mu^{\epsilon} \frac{\Gamma(\epsilon)}{\Gamma\left(\frac{3}{2}+\frac{\epsilon}{2}\right)}(i \eta)^{\epsilon}
$$


e portanto

$$
C=\frac{1}{48 \pi^{2}} \frac{1}{\epsilon}
$$

ou seja, pela equação (D.8), temos que

$$
\left(g^{\prime}\right)=\varepsilon^{\mu \nu \alpha} p_{\alpha} \frac{1}{48 \pi^{2}} \frac{1}{\epsilon}+\text { parte finita }
$$

Porém vemos que o diagrama $(f)$ carrega um fatorr $\frac{\left(i e^{2}\right)^{2}}{2 !} 2^{3}=-2 e^{4}$, enquanto que para $(g)$ temos $\frac{\left(i e^{2}\right)^{2}}{4 !} 4 !=e^{4}$. Portanto, somando as colaborações das partes divergentes dos dois diagramas, temos que

$-2 e^{4}\left(\right.$ parte div. de $\left.\left(\mathrm{f}^{\prime}\right)\right)+e^{4}\left(\right.$ parte div. de $\left.\left(\mathrm{g}^{\prime}\right)\right)=-\frac{2 e^{4}}{96 \pi^{2}} \frac{1}{\epsilon} \varepsilon^{\mu \nu \rho} p_{\rho}+\frac{e^{4}}{48 \pi^{2}} \frac{1}{\epsilon} \varepsilon^{\mu \nu \rho} p_{\rho}=0$.

De onde podemos concluir que $B=0 \mathrm{em}$ (1.49), ou seja, que não temos renormalização para a função de onda do $A_{\mu}$ (ou, equivalentemente do parâmetro de Chern-Simons). 


\section{Apêndice E}

\section{Identidades de Ward}

Vamos neste apêndice discutir com um pouco mais de detalhe as identidades de Ward para o modelo em questão, e como estas identidades fornecem relações entre as constantes de renormalização .

Como já foi dito no capíulo I, as identidades de Ward[42, 43] relacionam as funções de vértice próprias 1-PI, $\Gamma^{\mu}\left(q ; p, p^{\prime}\right)$ e $\Gamma^{\mu \nu}\left(q, k ; p, p^{\prime}\right)$, trilinear e quadrilinear com respeito ao vértice de Chern-Simons, com as funções de vértice de dois e três pontos, $\Gamma(p) \mathrm{e}$ $\Gamma_{\nu}\left(k, p+q, p^{\prime}\right)$, respectivamente. Explicitamente estas relações são dadas por

$$
\begin{aligned}
q^{\mu} \Gamma_{\mu}\left(q ; p, p^{\prime}\right) & =-e \mu^{\frac{\epsilon}{2}}\left[\Gamma\left(p^{\prime}\right)-\Gamma(p)\right] \\
q^{\mu} \Gamma_{\mu \nu}\left(q, k ; p, p^{\prime}\right) & =-e \mu^{\frac{\epsilon}{2}}\left[\Gamma_{\nu}\left(k, p+q, p^{\prime} p^{\prime}\right)-\Gamma_{\nu}\left(k, p, p^{\prime}-q\right)\right] .
\end{aligned}
$$

Sendo estas relações válidas em cada ordem de perturbação vamos considerar, como exemplo, as três primeiras correções relativas às funcões de vértice de três pontos $\Gamma_{\mu}\left(q ; p, p^{\prime}\right)$, vistas na figura E.1.

Para o caso (a), baseados em (1.16), podemos escrever

$$
\Gamma_{0}^{\mu}\left(p^{\prime}, p\right)=-i e \mu^{\frac{\epsilon}{2}}\left(p^{\prime}+p\right)^{\mu}
$$

multiplicando por $q_{\mu}=\left(p^{\prime}-p\right)_{\mu}$ temos

$$
\begin{aligned}
q_{\mu} \Gamma_{0}^{\mu}\left(p^{\prime}, p\right) & =-i e \mu^{\frac{\varepsilon}{2}}\left(p^{\prime}-p\right)_{\mu}\left(p^{\prime}+p\right)^{\mu} \\
& =-e \mu^{\frac{\epsilon}{2}}\left[\Gamma_{0}^{(2)}\left(p^{\prime}\right)-\Gamma_{0}^{(2)}(p)\right]
\end{aligned}
$$

na equação acima a função de vértice de ordem zero, $\Gamma_{0}^{(2)}$, é a função própria de dois pontos (que foi formalmente definida no capítulo II) e é dada por $\Gamma_{0}^{(2)}(p)=-[\Delta(p)]^{-1}$. Assim (E.4) fica da forma

$$
q_{\mu} \Gamma_{0}^{\mu}\left(p^{\prime}, p\right)=-e \mu^{\frac{e}{2}}\left[\Delta_{0}^{(-1)}\left(p^{\prime}\right)-\Delta_{0}^{(-1)}(p)\right] .
$$



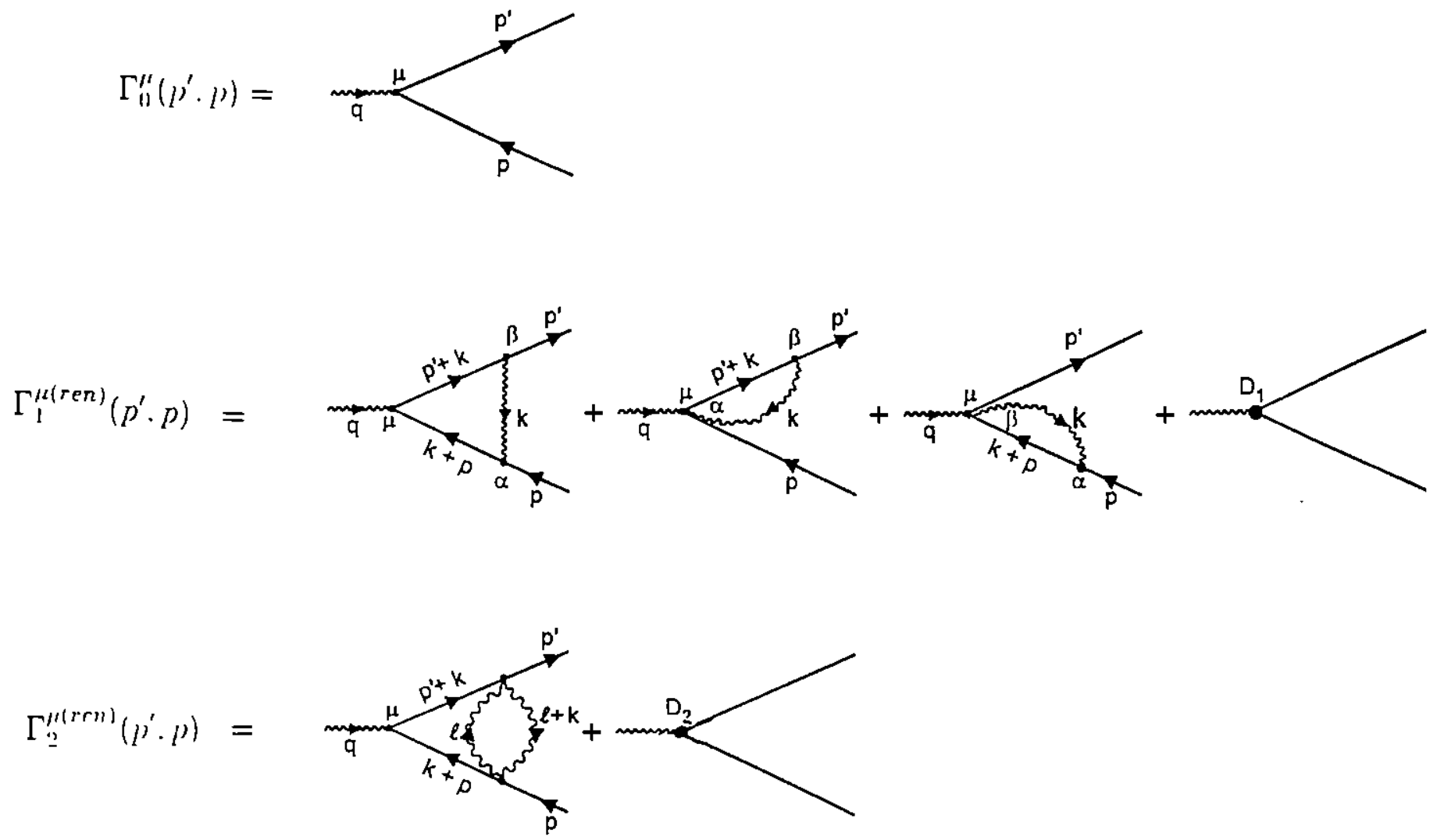

Figura E.1: Funcões de vértice com correção até segunda orden. Em todos os diagramas temos $q=p^{\prime}-p$. Sendo que $D_{1}$ e $D_{2}$ são contratermos necessários para tornar finitas $\Gamma_{1}^{\mu}$ e $\Gamma_{2}^{\mu}$ respectivamente. 
Para o caso (b) podemos escrever que

$$
\begin{aligned}
\Gamma_{1}^{\mu(r e n)}\left(p^{\prime}, p\right) & =\left(-i e \mu^{\frac{\epsilon}{2}}\right)^{3} \frac{3 !}{3 !} \int \mathcal{D} k \frac{i}{(p+k)^{2}} \frac{i}{\left(p^{\prime}+k\right)^{2}} \varepsilon^{\alpha \beta \rho} \frac{k_{\rho}}{k^{2}}\left(p+p^{\prime}+2 k\right)^{\mu}(2 p+k)_{\alpha}(2 p+k)_{\beta} \\
& +2\left(-i e \mu^{\frac{\epsilon}{2}}\right)\left(i e^{2} \mu\right) \int \mathcal{D} k\left[\varepsilon^{\mu \beta \rho} \frac{k_{\rho}}{k^{2}} \frac{i}{\left(p^{\prime}+k\right)^{2}}\left(2 p^{\prime}+k\right)_{\beta}+\varepsilon^{\alpha \mu \rho} \frac{k_{\rho}}{k^{2}} \frac{i}{(p+k)^{2}}(2 p+k)_{\alpha}\right] \\
& -i e \mu^{\frac{\epsilon}{2}} D_{1}\left(p^{\prime}-p\right)^{\mu}
\end{aligned}
$$

na qual a medida $\mathcal{D} k=\frac{d^{d} k}{(2 \pi)^{d}}$. Fazendo o produto de (E.6) com $q_{\mu}=\left(p^{\prime}-p\right)_{\mu}$ e escrevendo $\left(p^{\prime}-p\right)_{\mu}\left(p+p^{\prime}+2 k\right)^{\mu}=\left(p^{\prime}+k\right)^{2}-(p+k)^{2}$ temos, após alguma álgebra, que

$$
\begin{aligned}
& q_{\mu} \Gamma_{1}^{\mu(\tau e n)}\left(p^{\prime}, p\right)=\left(-i e \mu^{\frac{\epsilon}{2}}\right)^{3} \int \mathcal{D} k \varepsilon^{\alpha \beta \rho} \frac{k_{\rho}}{k^{2}}\left[\frac{\left(2 p^{\prime}+k\right)_{\alpha}\left(2 p^{\prime}+k\right)_{\beta}}{\left(p^{\prime}+k\right)^{2}}-\frac{(2 p+k)_{\alpha}(2 p+k)_{\beta}}{(p+k)^{2}}\right] \\
& -i e \mu^{\frac{e}{2}} D_{1}\left(p^{\prime 2}-p^{2}\right)
\end{aligned}
$$

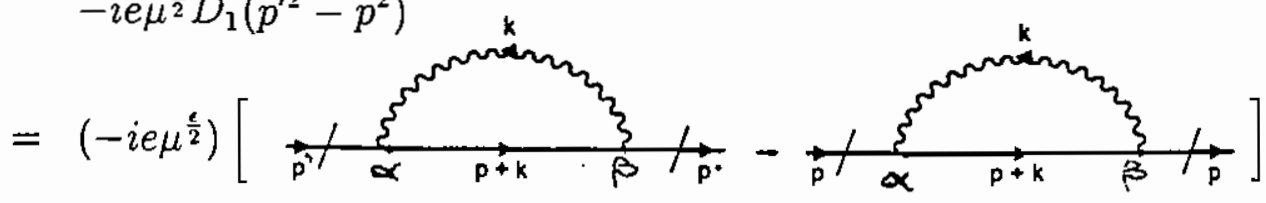

$$
\begin{aligned}
& -i e \mu^{\frac{\epsilon}{2}} D_{1} p^{\prime 2}+i e \mu^{\frac{\epsilon}{2}} D_{1} p^{2},
\end{aligned}
$$

ou ainda, se tomarmos $D_{1}=A_{1}$, temos

$$
\left.q_{\mu} \Gamma_{1}^{\mu(r e n)}\left(p^{\prime}, p\right)=\left(-e \mu^{\frac{\varepsilon}{2}}\right)\left[\Gamma_{1}^{(r e n)}\left(p^{\prime}\right)-\Gamma_{1}^{(r e n)}(p)\right]\right]
$$

Para o caso (c) a amplitude $\Gamma_{2}^{\mu}\left(p^{\prime}, p\right)$ é dada por

$$
\begin{aligned}
\Gamma_{2}^{\mu(\text { ren })}\left(p^{\prime}, p\right)= & \left(-i e \mu^{\frac{\epsilon}{2}}\right)^{3}\left(i e \mu^{\epsilon}\right)^{2} \frac{4}{2 !} \int \mathcal{D} k \Theta(k) \frac{i}{\left(p^{\prime}+k\right)^{2}} \frac{i}{(p+k)^{2}}\left(2 k+p+p^{\prime}\right)^{\mu} \\
& -\left(i e \mu^{\frac{\epsilon}{2}}\right) D_{2}\left(p^{\prime}+p\right)^{\mu}
\end{aligned}
$$

na qual $\Theta(k)$ representa o "loop" composto pelos propagadores de Chern-Simons (este "loop" será calculado oportunamente, porém, para o nosso intuito no momento, podemos representá-lo desta forma). Fazendo novamente o produto de (E.9) por $\left(p^{\prime}-p\right)_{\mu}$ temos

$$
\begin{aligned}
& q_{\mu} \Gamma_{2}^{\mu(\tau e n)}\left(p^{\prime}, p\right)=\left(-2 i e \mu^{\frac{\epsilon}{2}}\right)\left(i e \mu^{\epsilon}\right)^{2} \int \mathcal{D} k \Theta(k) i\left[\frac{i}{(p+k)^{2}}-\frac{i}{\left(p^{\prime}+k\right)^{2}}\right] \\
& -e \mu^{\frac{\epsilon}{2}}\left(i p^{\prime 2} D_{2}-i p^{2} D_{2}\right)
\end{aligned}
$$

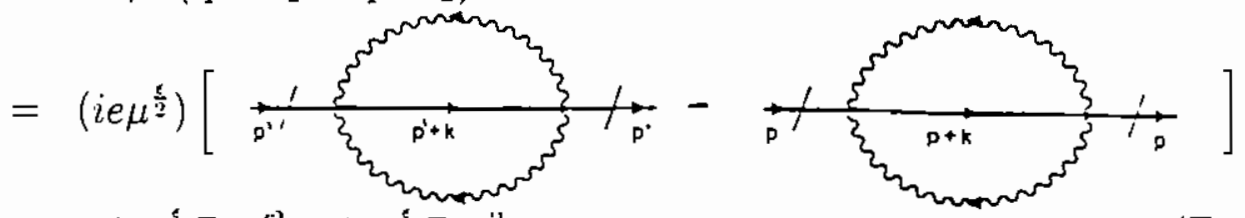

$$
\begin{aligned}
& -i e \mu^{\frac{6}{2}} D_{2} p^{\prime 2}+i e \mu^{\frac{6}{2}} D_{2} p^{2} \text {, }
\end{aligned}
$$




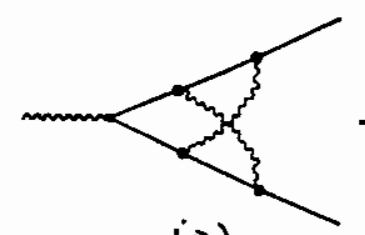

(i)
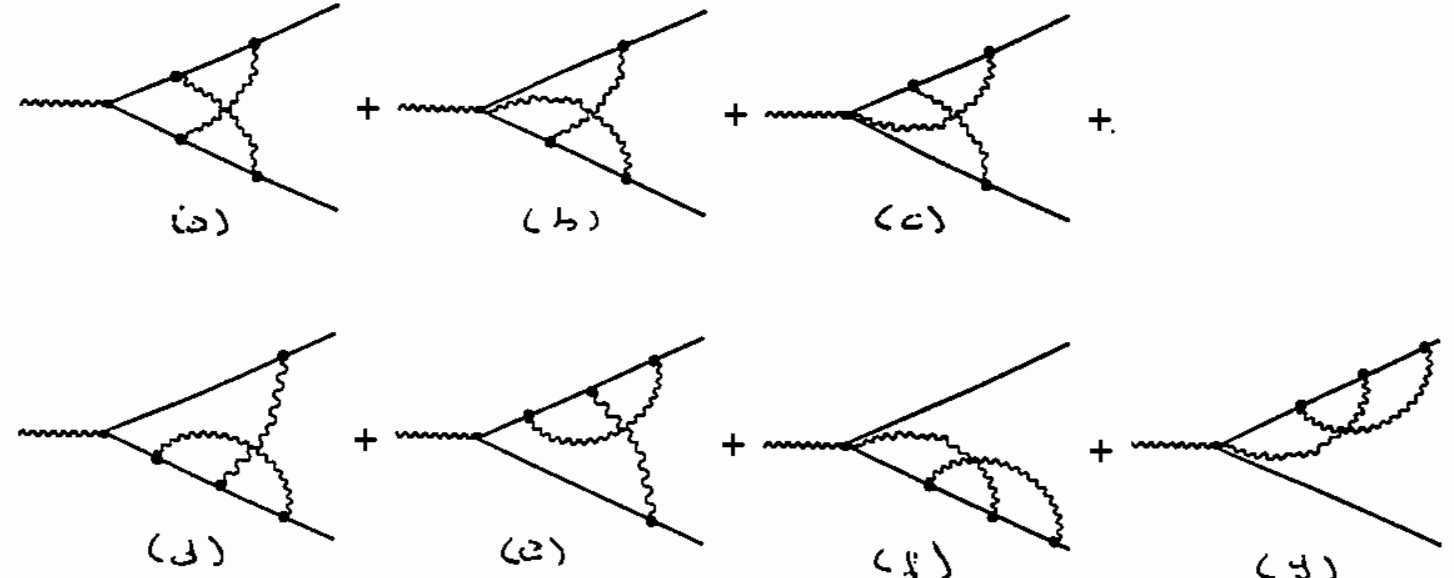

(y)

Figura E.2:

ou ainda, escolhendo $D_{2}=A_{2}$ temos que

$$
q_{\mu} \Gamma_{2}^{\mu(\tau e n)}\left(p^{\prime}, p\right)=\left(-e \mu^{\frac{\epsilon}{2}}\right)\left[\Gamma_{2}^{(\tau e n)}\left(p^{\prime}\right)-\Gamma_{2}^{(\tau e n)}(p)\right] .
$$

As expressões (E.5), (E.8) e (E.11) confirmam a identidade de Ward e consequentemente garantem a relação

$$
A=D \text {. }
$$

Para melhor ilustrar esta situação vamos verificar explicitamente, para um caso particular, que as partes divergentes satisfazem a identidade de Ward. Vamos considerar a família mostrada na figura (E.2).

Todos os diagramas mostrados na família da figura (E.2) possuem, por simples contagem de potências, divergência linear. Porém pode-se constatar que se efetuarmos as deridas constrações de Lorentz. apenas os diagramas (f) e (g) são efetivamente divergentes. mais propriamente. logaritimicamente divergentes. Estes dois diagramas podem ser vistos com mais detalhes na figura (E.3) sendo,

$$
(f)_{\beta}=-i \iint \mathcal{D} k \int \mathcal{D} q \varepsilon^{\mu \nu \rho} \varepsilon^{\alpha \beta \gamma} \frac{k_{\rho} q_{\gamma}(2 p+k)_{\mu}(2 p+2 k+q)_{\alpha}(2 p+2 q+k)_{\nu}}{k^{2} q^{2}(p+k)^{2}(p+k+q)^{2}(p+q)^{2}},
$$

ou ainda. fazendo as contrações de Lorentz e já incluindo o fator conbinatorial, temos que a parte divergente de $(f)_{3}$ será

$$
(f)_{3}^{(d i v)}=-8 i p_{\mu} \int \mathcal{D} k \int \mathcal{D} q \varepsilon^{\mu \nu \rho} \Xi^{\alpha \beta \gamma} \frac{k_{\rho} k_{\alpha} q_{\gamma} q_{\nu}}{\left[k^{2}\right]^{2}\left[q^{2}\right]^{2}[k+q]^{2}} .
$$




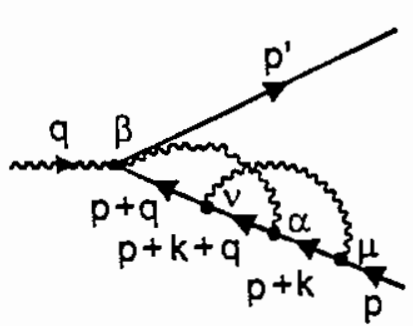

()$_{E}$

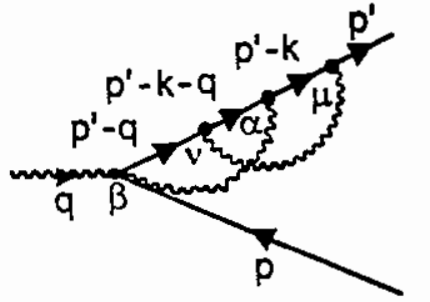

(g) $\beta$

Figura E.3:

Fazendo a integral em $q$ e em $k$, usando o procedimento idêntico ao aplicado nos outros gráficos, temos

$$
(f)_{\beta}^{(d i v)}(p)=-\frac{i}{24 \pi^{2}} \frac{p_{\beta}}{\epsilon} .
$$

Para o diagrama $(g)_{\beta}$, obteremos um resultado equivalente, ou seja

$$
(g)_{3}^{(d i v)}\left(p^{\prime}\right)=-\frac{i}{24 \pi^{2}} \frac{p_{\beta}^{\prime}}{\epsilon} .
$$

Portanto teremos que

$$
\begin{aligned}
q^{\beta}\left[(f)_{\beta}^{(d i v)}(p)+(g)_{\beta}^{(d i v)}\left(p^{\prime}\right)\right] & =-\frac{i}{12 \pi^{2}} \frac{1}{\epsilon}\left(p^{2}-p^{2}\right) \\
& =\left(-\frac{i}{12 \pi^{2}} \frac{1}{\epsilon} p^{2}\right)-\left(-\frac{i}{12 \pi^{2}} \frac{1}{\epsilon} p^{2}\right)
\end{aligned}
$$

que. ao ser comparada com o resultado obtido para $C_{3}$, equação (2.103) termos

$q^{\beta}\left[(f)_{3}^{(d i v)}(p)+(g)_{3}^{(d i v)}\left(p^{\prime}\right)\right]=[$

verificando. para este exemplo, que as partes divergentes satisfazem a identidade de Ward (E.1).

Como vimos até aqui podemos concluir que famílias específicas das funções de vértice de três pontos relacionan-se, via identidade de Ward a um diagrama da função de vértice 
$[<<<<<]-[\cap]$

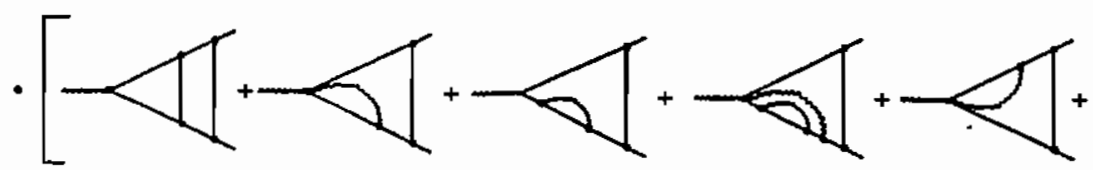

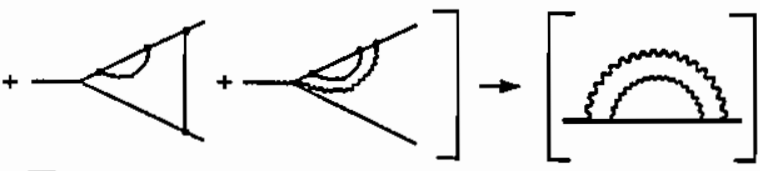

$[\Delta+\infty+\infty+\infty+$

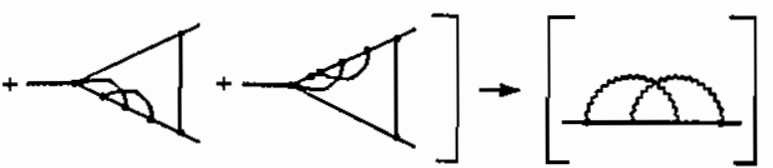

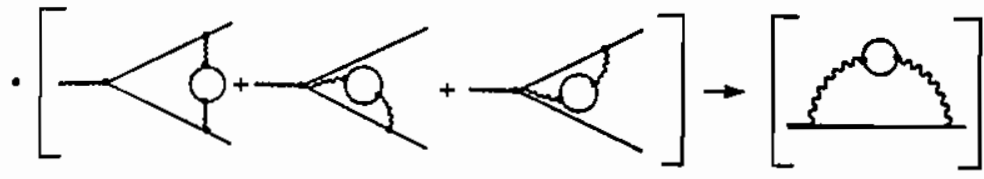

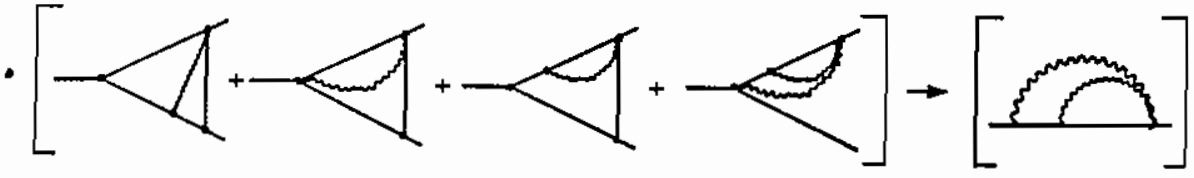

- $[\longrightarrow[+\longrightarrow[+\longrightarrow$

- $[-1\}$

Figura E.4: Famílias de Diagramas que satisfazem a identidade de Ward 


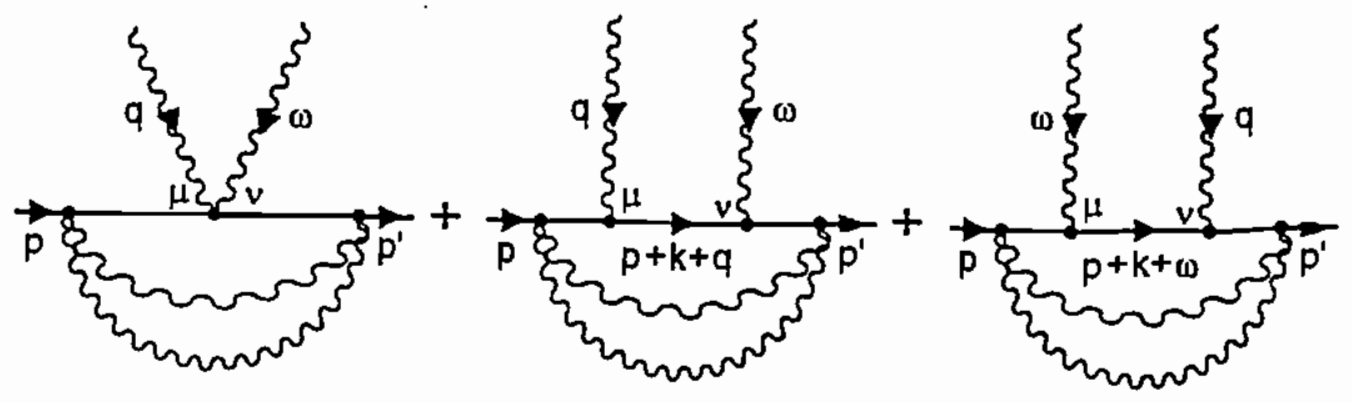

Figura E.5: Família da função de vértice de quatro pontos. Para cada diagrama temos $p^{\prime}=p+w+q$.

de dois pontos. Algumas destas famílias de três pontos e os respectivos diagramas de dois pontos podem ser vistos na figura (E.4).

Como ilustração para a segunda identidade de Ward, equação (E.2), vamos analisar a família mostrada na figura (E.5) Sendo que na figura (E.5) o "loop" composto da linha de Chern-Simons corresponde ao diagrama $\Theta(p)$, figura (2.2), cujo resultado pode ser visto em (2.24).

A função $\Gamma_{\mu \nu}\left(q, w, p^{\prime}, p\right)$ correspondente aos três diagramas é dada, analiticamente por

$$
\begin{aligned}
\Gamma_{\mu \nu}\left(q, w, p^{\prime}, p\right)= & i e^{6} \mu^{3 \epsilon} \int d^{d} k \frac{1}{(k+p)^{2}}\left[4 g_{\mu} \nu-\frac{2(2 p+2 k+q)_{\mu}\left(2 p^{\prime}+2 k-w\right)_{\nu}}{(k+q+p)^{2}}\right. \\
& \left.-\frac{2(2 p+2 k+w)_{\nu}\left(2 p^{\prime}+2 k-q\right)_{m} u}{\left(p^{\prime}+k-q\right)^{2}}\right] \frac{\Theta}{\left(p^{\prime}+k\right)^{2}} .
\end{aligned}
$$

Da qual podemos ver que

$$
\begin{aligned}
q^{\mu} \Gamma_{\mu \nu}\left(q . w, p^{\prime}: p\right)= & i e^{6} \mu^{3 \epsilon} \int d^{d} k \frac{1}{(k+p)^{2}}\left[4 q_{\nu}-\frac{2\left[2(p+k) \cdot q+q^{2}\right]}{(p+k)^{2}+2(p+k) \cdot q+q^{2}}\left(2 p^{\prime}+2 k-w\right)_{\nu}\right. \\
& \left.-\frac{2\left[-2(p+k) \cdot q+q^{2}\right]}{\left(p^{\prime}+k\right)^{2}-2(p+k) \cdot q+q^{2}}(2 p+2 k+w w)_{\nu}\right] \frac{\Theta}{\left(p^{\prime}+k\right)^{2}} .
\end{aligned}
$$

ou ainda: tendo em vista a conservação dos momentos $p^{\prime}=p+w+q$, e a forma para $\Gamma_{2}^{\mu}\left(p^{\prime}, o\right)$ em (E.9), podemos escrever por fim que

$$
q^{\mu} \Gamma_{\mu \nu}\left(q, w \cdot p^{\prime} ; p\right)=-e \mu^{\frac{\epsilon}{2}}\left[\Gamma_{\nu}\left(p^{\prime}, p^{\prime}-w\right)-\Gamma_{\nu}(p+w, p)\right]
$$


verificando a identidade de Ward entre as funções de quatro e três pontos. Da equação acima podemos ver que

$$
E=D,
$$

quando forem introduzidos os contratermos necessários para renormalizar $\Gamma_{\mu \nu}$ e $\Gamma_{\mu}$ acima. 


\section{Bibliografia}

[1] J. M. Leinaas e J. Myrheim, Il Nuovo Cimento 37, 1 (1977).

[2] d. C. Tsui, H. L. Stormer and A. C. Gossard, Phys. Rev. Lett. 48, 1559 (1982).

[3] F. D. M Haldane, Phys. Rev. Lett. 51, 605 (1983).

[4] B. I. Halperin, Phys. Rev. Lett. 52,11585 (1983).

[5] R. B. Laughlin, Phys. Rev. bf B23, 3383 (1984).

[6] J. D. Lykken, J. Sonnenschein and N. Weiss, Int. Jour. Mod. Phys. A6, 5155 (1991).

[7] P. B. Wiegmann, Phys. Rev. Lett. 60, 821 (1988).

[8] R. B. Laughlin, Phys. Rev. Lett. 60 2677, (1988).

[9] Y. H. Chen, F. Wilczek, E. Witten, and B. J. Halperin, Int. Jour. Mod. Phys. B3 1942 (1989).

[10] A. P. Balachandran, E. Ercolessi, G. Morandi and A. M Srivastava, World Scientific, Singapore, (1990).

[11] F. Wilczek, Phys. Rer. Lett. 48, 1144 (1982); F. Wilczek. Phys. Rer. Lett. 49, 957 (1982).

[12] Y. Aharonov and H. Bohm, Phys. Rer. 115, 485 (1959).

[13] D. P. Arovas, J. R. Schrieffer and F. Wilczek, Physs. Rer. Lett. 53, 772 (1984).

[14] J. F. Schonfeld, Nucl. Physs. B185, 157 (1981).

[15] S. Deser, R. Jackiw and S. Templeton, Ann. Phys. 140. 372 (1982).

[16] W. Chen and M. Li, Phys. Rev. Lett. 70, 884 (1993).

[17] W. Chen, Nucl. Phys. B435, 689 (1995). 
[18] V. S. Alves, M. Gomes, A. J. da Silva, S. V. L. Pinheiro, The Perturbative GrossNeveu Model Coupled to a Chern-Simons Field: A Renormalization Group Study (1998) Aceito para publicação em Phys. Rev. D.

[19] Van. Sérgio Alves, Análise Perturbativa para Férmions com Auto-Interação Quártica Acoplados com um Campo de Chens-Simons, (1988) Tese de doutorado - IFUSP.

[20] P. K. Townsend, Nucl. Phys. B118, 199 (1977).

[21] R. D. Pisarski, Phys. Rev. Lett. 48, 574 (1982).

[22] D. G. C. Mckeon and G. Tsoupros, Phys. Rev. D46, 1794 (1992).

[23] M. Blume, V. J. Emery and R. B. Griffiths, Phys. Rev. A4, 1071 (1971).

[24] D. J. Amit and E. Rabinovici, Nucl. Phys. B257, 371 (1984).

[25] C. A. Aragão de Carvalho, Nucl. Phys. B119, 401 (1976).

[26] G. N. J. Ananos and N. F. Svaiter, XVII Encontro Nacional de Partículas e Campos, (1996).

[27] C. R. Hagen, Ann. Phys. (NY) 157 (1984) 342.

[28] S. Weinberg, The Quantum Theory of Fields, Cambridge Lniversity, (1995);

[29] M. E. Peskin and D. V. Schroeder, An Intruduction to Quantum Field Theory, Addison-Wesley Publishing Company, (1996);

[30] R. Jackiw and S. Templeton, Phys. Rev. D23, 2291 (1981);

[31] I. V. Tyutin and Vad. Yu. Zeitlin, "Gauge Inariance of $Q E D_{2+1}$ ", hep-th/9711137;

[32] M. O. C. Gomes, Apostila do Curso de Introdução à Teoria Quântica de Campos, I e II, IFUSP, (1998);

[33] J. Collins, Renormalization, Cambridge University Press; (1985);

[34] S. Deser, R. Jackiw, and S. Templeton, Phys. Rev. Lett. 48, 975 (1982); Ann. Phys. 140, 372 (1992);

[35] J. R. S. Nascimento, Estudo da Teoria de Chern-Simons no Gauge de Coulomb até um Loop, Dissertação de Doutorado, IFUSP, (1995).

[36] W. Pauli, Niels Bohr and Development of Physics, (Pergamon Press, London), 30, (1995), G. Luders, Ann. Phys. (NY) 2, 1 (1957). 
[37] C. G. Bollini and J. J. Giambiagi, Phys Lett 40B, 566 (1972); G. 't Hooft and M. Veltman, Nucl. Phys. B44, 189 (1972) ; J. f. Ashmore, Lett. Nuovo Cimento 4, 289 (1972).

[38] N. N. Bogoliubov, and O. Parakiok, Acto Math. 97, 227 (1957); N. N. Bogoliubov, and D. V. Shirkov, Introduction to the Theory of Quantized Fields (Wiley, New York) (1980);

[39] K. Happ, Comm. Math. Phys. 2, 301 (1966);

[40] W. Zimmermann, Lectures on Elementary Particles and Quantum Field Theory, Eds. S. Deser, M. Grisary and M. Pendloton (M.I.T., Cambridge, Mass) (1970).

[41] R. S. Willey, Phys. Rev. D48, 2877 (1993); T. Gherghota, Phys. Rev. D50, 5985 (1994).

[42] E. Spper, Renormalization Theory, Proceedings of International School Ettore Majorana, Erice 1975, edited by G. Velo and A. S. Wightman, D. Reidel Publishing Co, (1976);

[43] J. C. Ward, Phys. Rev 78, 182 (1950).

[44] Y. Takahashi, IL Nuovo Cimento, 6, 371 (1957).

[45] M. Abramowitz and A. Stegua, Handbook of Mathematical Functions, Dover Publications, (1970); I. S. Gradshteyn and I. M. Ryzhik, it Table of Integrals, Series and Products, Academic Press, (1963).

[46] G. t'Hooft, Nucl. Phys. B61, 455 (1973); S. Weinberg, Phys. Rev. D8, 3497 (1973).

[47] S. Coleman and B. Hill, Phys. Lett. 159B, (1985);

[48] G. W. Semenoff, P. Sodano, and Yong-Shi Wu, Phys. Rev. Lett. 62, 715 (1989); V. P. Spiridonov and F. V. Tkachov, Phys. Lett. B260, 109 (1991); A. Khare, R. MacKenzie, and M. B. Paranjape, Phys. Lett. B343, 239 (1995): Hsien-Chung Kao, Mod. Phys. Lett. A12, 763 (1997);

[49] D. J. Gross, Applications of the Renorlization Group to High-Energy Physics, Proceedings of Les Houches, Session XXVIII, 1975, edited by Roger Balian and Jean Zinn-Justin, North-Holland Publishing Co, (1996);

[50] S. Weinbeg. Phys. Rev. 118, 838 (1960);

[51] W. Siegel, Phys. Lett. 84B, 193 (1979); Phys. Lett. 94B, 37 (1980); C. M. Hull, and P. K. Townsend, Phys. Lett. 191B, 513 (1987); M. Bos, Ann. Phỳs. 181, 177 (1988); C. P. Martin, Phys. Lett. 241B, 513 (1990); 
[52] Wei Chen, G, W. Semenoff and Yong-Shi Wu, Mod. Phys. Lett. A, Vol. 5, (1990);

[53] L. V. Avdeev, G. V. Grigoryev, and D. I. Kazakov, Nucl. Phys. B382, 561, (1992);

[54] W. Zimmermann, Comm. Math. Phys. 15, 208 (1969);

[55] K. G. Wilson, Phys. Rev. D3,1818 (1971); 